\title{
A Study of the Uptake of $\mathrm{Cu}^{2+}$ by Calcium Silicate by Batch and Continuous Reactors for Potential Commercialisation
}

\author{
By \\ Giancarlo Mario Barassi Infante \\ VICTORIA UNIVERSITY OF WELLINGTON \\ Te Whare Wānanga o te Ūpoko o te Ika a Māui

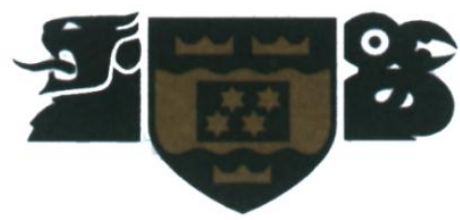

A thesis

submitted to the Victoria University of Wellington

in fulfilment of the requirements for the degree of

Doctor of Philosophy

Victoria University of Wellington 


\section{Abstract}

This study presents a significant advancement in the understanding of the uptake of $\mathrm{Cu}^{2+}$ by nanostructured calcium silicate (NCaSil) and to develop a strategy of using it in a continuous manner using packed columns. The NCaSil structure consists of micro-sized agglomeration of nanometre-sized platelets of calcium silicate. This arrangement grants the material a large surface area of 400 to $600 \mathrm{~m}^{2} \mathrm{~g}^{-1}$.

The kinetics and thermodynamics of the adsorption of $\mathrm{Cu}^{2+}$ onto NCaSil in batch were studied at temperatures ranging from 277 to $333 \mathrm{~K}$. The reaction between $\mathrm{Cu}^{2+}$ and $\mathrm{NCaSil}$ occurred rapidly, being endothermic and exhibiting an increase in the entropy meaning that the adsorption process became more spontaneous when the temperature was increased. Furthermore, the uptake resulted in the formation of copper sulfate hydroxide minerals in the form of $\mathrm{Cu}_{4}(\mathrm{OH})_{6} \mathrm{SO}_{4} \cdot n \mathrm{H}_{2} \mathrm{O}$, where $n$ is equal to 2 for wroewolfeite, 1 for posnjakite and 0 for brochantite. Using powder X-ray diffraction and scanning electron microscopy it was proven that at temperatures between 293 and $313 \mathrm{~K}$ wroewolfeite and posnjakite were intermediates in the formation of brochantite. Specifically at high temperatures of $333 \mathrm{~K}$ and $\mathrm{Cu}^{2+}$ concentrations higher than $15.7 \mathrm{mmol} \mathrm{L}^{-1}$ the reaction proceeded directly to the formation of the thermodynamically stable compound brochantite. A kinetic study of the crystal growth was carried out using powder-XRD which showed that the rate determining step towards the formation of brochantite is the nucleation of $\mathrm{SO}_{4}{ }^{2-}$. Additionally, a value for the activation energy of $42 \mathrm{~kJ} \mathrm{~mol}^{-1}$ using powder-XRD data was obtained for the formation of the crystallographic plane 420 in the brochantite crystal.

A sample of a real mining waste was collected and analysed. Based on this sample an emulated waste was generated. The NCaSil was tested for the uptake of $\mathrm{Cu}^{2+}$ ions from this emulated mining waste, showing that the use of NCaSil is feasible at $\mathrm{pH}$ values greater than 3 . The production and use of NCaSil may be coupled to existing mining waste treatment processes in order to remove dissolved copper from solution and produce a copper rich solid as the by-product.

NCaSil was packed inside a conventional axial flow column and a radial flow column, which was developed as part of this project. The former proved to be impractical due to a large pressure drop through the column, while the latter was impractical due to short operational times before breakthrough. Nonetheless, the radial flow column was operated by immersion in a tank exhibiting similar kinetics of copper ions uptake to those observed in batch processes. Therefore, the scale-up of this process was proposed including the necessary equations keeping the ratio of the tested radial flow column. 


\section{Acknowledgements}

I would like to thank the support given to me throughout my $\mathrm{PhD}$ by my wife Katherina, the $\mathrm{PhD}$ stood us apart for 2 years but we made it through with patience and perseverance. I will always remember the help and support from my entire family and friends in Chile, without them it would have been extremely difficult to accomplish everything that I have achieved in my life. All my success is dedicated to them.

I would like to give thanks to Dr. Thomas Borrmann for accepting me into his research group allowing me to study in a world-class university in New Zealand. His support during my PhD was crucial for me, correcting my thesis even after his departure from Victoria University of Wellington. I would also like to thank Dr. Mathew Cairns who proof read my thesis and lent part of his time to me to discuss my questions about the project. Additionally, I would like to thank Prof. Jim Johnston who kindly accepted to supervise me. His help was important since Dr. Borrmann's departure in late 2011.

Since 2009 I have met wonderful people like: Prof. Joachim Kinkel, who gave me professional advice which I have taken into account; Martin Klimsa, who became a very close friend and made me feel I always had someone close to talk with; Omar Alsaqer, with whom I learn about the muslim culture and enjoyed many meals and coffees, I always felt his support which helped cope when I felt homesick; Dr. Carla Fonseca, she always was very glad to assist my wife and me. I would sincerely like to thank my entire research group with whom I enjoyed working; Andrea Kolb, Thomas Nilson; Mathias Herzhog, Eldon Tate, Maria Parry, Kerstin Lucas, Michael Welter, Mohammed Alzeer, Mahroo Fallah and numerous international students who visited us. My most sincere thanks to Alan, Manu and Nick from the workshop who help built the RFC device and always were ready to assist me.

I would like to acknowledge the Comisión Nacional de Investigación en Ciencia y Tecnología from the Chilean government and Victoria University of Wellington for granting me a CONICYT-VUW 2009 PhD scholarship. My thanks also go to Curtis Gordon Scholarship for the grants received in 2010 and 2011. I would also like to acknowledge the faculty strategic research grants for the funds given to undertake experimental measurements in Germany. Finally, many thanks to CODELCO for granting a mining waste sample for this study and for allowing publishing the data in this thesis. 


\section{Table of Contents}

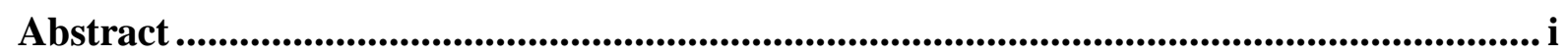

Acknowledgements ................................................................................................................ ii

Table of Contents .........................................................................................................................ii

List of Figures ................................................................................................................... vi

List of Tables.............................................................................................................................. $\mathbf{x v}$

Glossary .................................................................................................................................... xvii

Chapter 1 , Introduction ................................................................................................................... 1

1.1 Background information on New Zealand and Chile water availability and condition ... 1

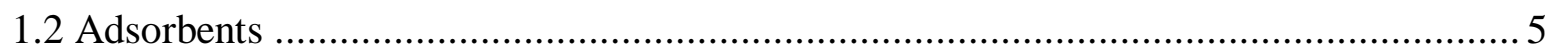

1.3 Nanostructured Calcium Silicate Hydrate (NCaSil) ................................................ 8

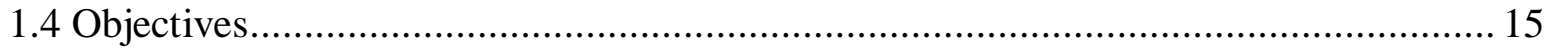

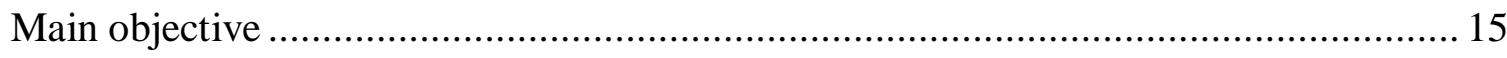

Chapter 2 , Experimental Procedure ............................................................................................ 16

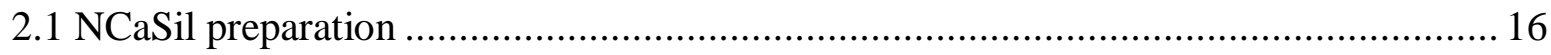

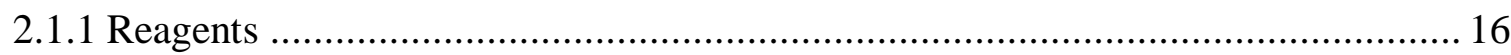

2.1.2 Procedure to manufacture NCaSil................................................................ 17

2.2 Kinetic studies on the adsorption $\mathrm{Cu}^{2+}$ in batch configuration ................................ 18

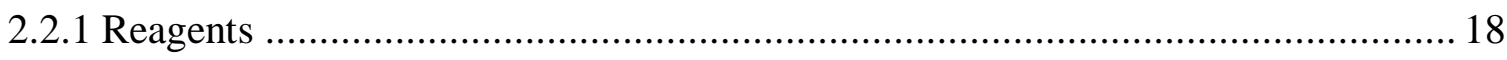

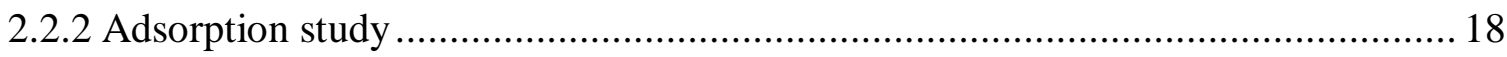

2.3 Study of the equilibrium of $\mathrm{Cu}^{2+}$ adsorption in batch configuration........................... 19

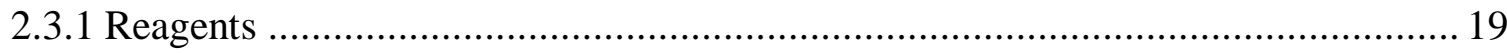

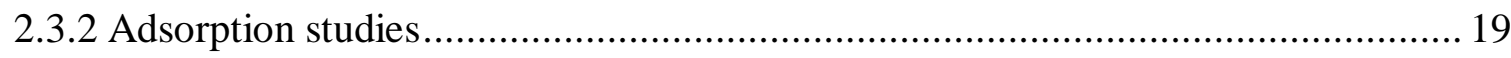

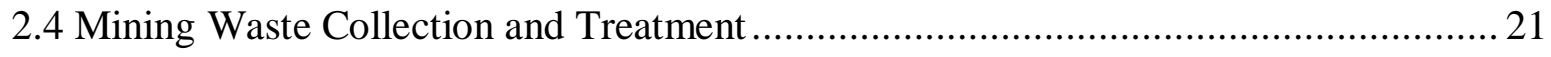

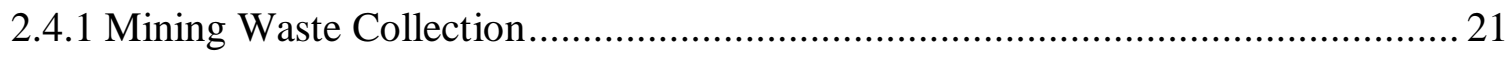

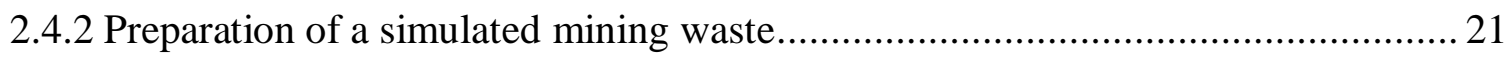

2.4.3 Emulated Mining waste Treatment. ................................................................ 22

2.5 Continuous adsorption studies .................................................................... 24 


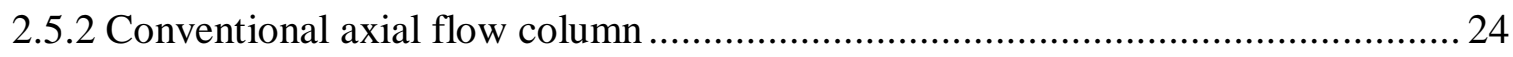

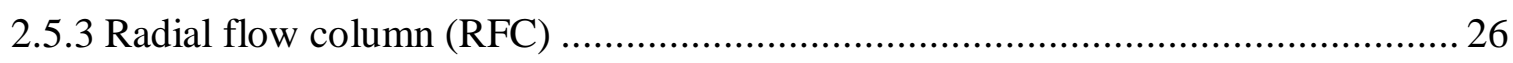

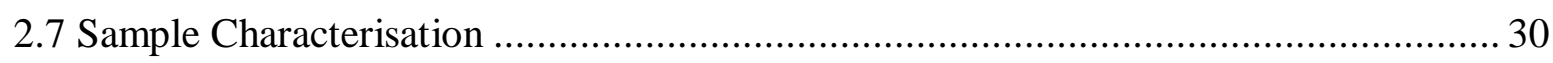

2.7.1 Liquid sample characterisation, $\mathrm{Cu}^{2+}$ sorption kinetic experiments ...................... 30

2.7.2 Liquid sample characterisation, equilibrium of the sorption of $\mathrm{Cu}^{2+} \ldots \ldots \ldots \ldots \ldots \ldots . . . . . . . .33$

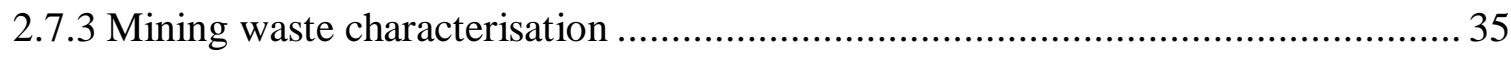

2.7.4 Characterisation of solid samples collected from kinetic and equilibrium studies of

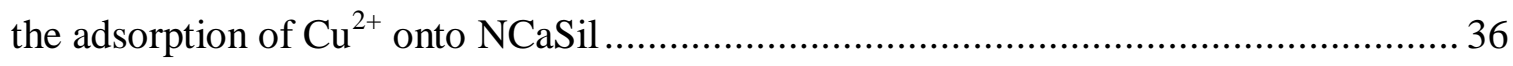

2.8 Statistical analysis and calculation of errors of the experimental data....................... 38

Chapter 3 , A study of the adsorption of $\mathrm{Cu}^{2+}$ ions onto NCaSil................................. 45

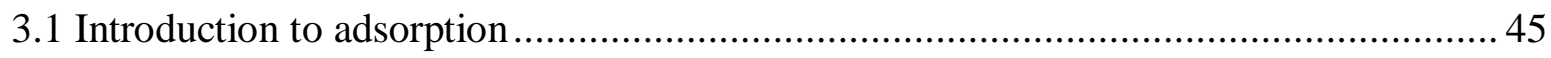

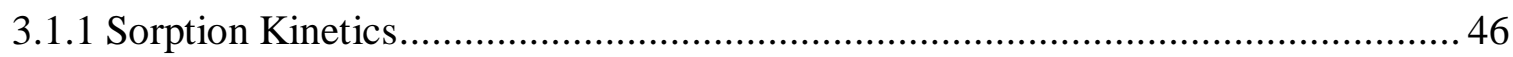

3.2 Results and discussion for the adsorption kinetic of $\mathrm{Cu}^{2+}$ onto $\mathrm{NCaSil} \mathrm{........................50}$

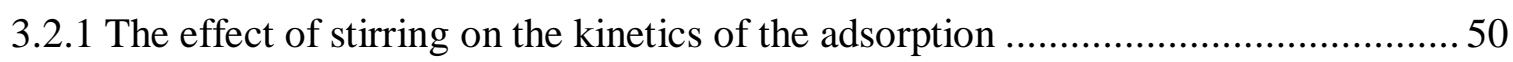

3.2.2 The effect of temperature on the kinetic of the adsorption of $\mathrm{Cu}^{2+}$ onto $\mathrm{NCaSil} \ldots . .52$

3.2.3 Kinetic study at different temperatures on the release of calcium ions ................. 55

3.2.4 Kinetic study on monomeric silica release during the uptake of $\mathrm{Cu}^{2+} \ldots \ldots \ldots \ldots \ldots \ldots . . . . . . .58$

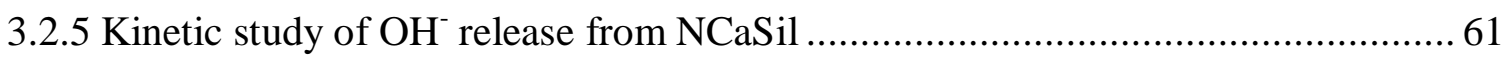

3.2.6 Powder-XRD study on the kinetic of crystal growth of copper minerals on the

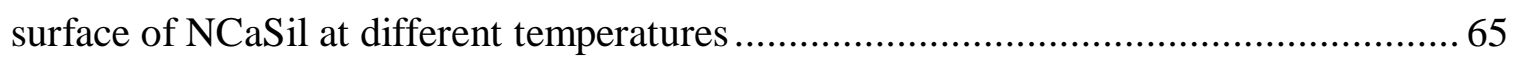

3.2.7 SEM and EDS study on the kinetics of $\mathrm{Cu}^{2+}$ adsorption and crystal growth .......... 87

3.3 Study of the equilibrium of the adsorption of $\mathrm{Cu}^{2+}$ onto $\mathrm{NCaSil} \mathrm{\ldots ........................} 95$

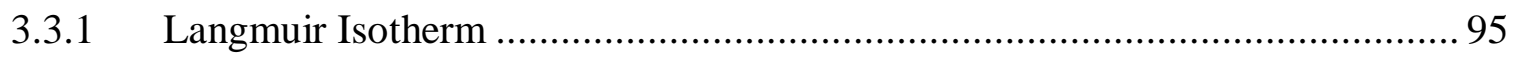

3.3.2 Freundlich Isotherm............................................................................ 96

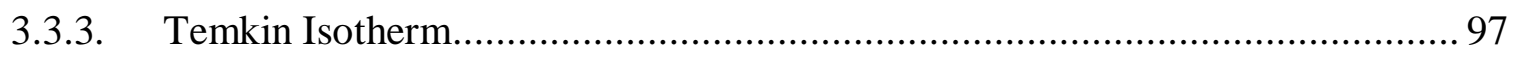

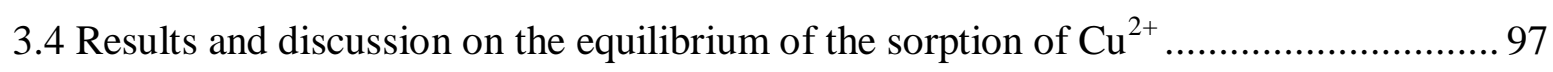

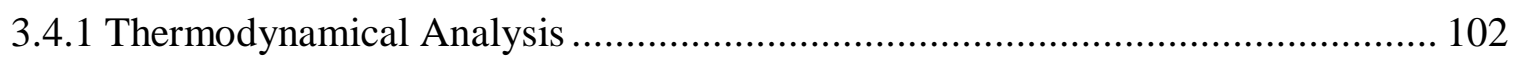

3.4.2 Powder-XRD study on the crystal growth at different temperatures at the

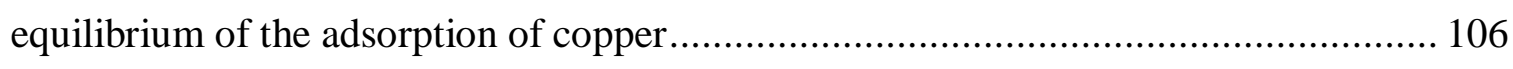

3.4.3 Study of the release of calcium at the equilibrium......................................... 109 
3.4.4 Study of the release of monomeric silica at the equilibrium

3.4.5 The effect of the initial $\mathrm{pH}$ of the solution on the amount of copper adsorbed and the release of $\mathrm{Ca}^{2+}$ and monomeric silica at the equilibrium.

3.5 Chapter Conclusions

Chapter 4 , Mining Waste Characterisation, Simulation and Treatment with NCaSil 123

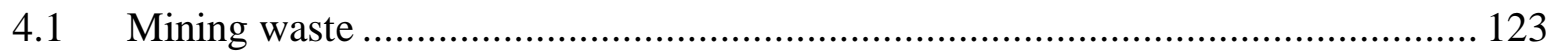

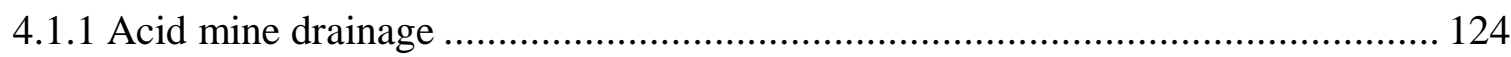

4.1.2 Technologies to address mining waste waters ............................................ 125

4.1.3 Weak acid production at El Teniente mine, Chile .......................................... 128

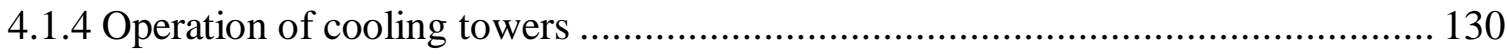

4.1.5 Chemical characterisation of the liquid phase, the supernatant .......................... 134

4.1.6 Characterisation of the solid phase: suspended particles. ............................... 136

4.1.7 Treatment of an emulated mining waste …................................................ 142

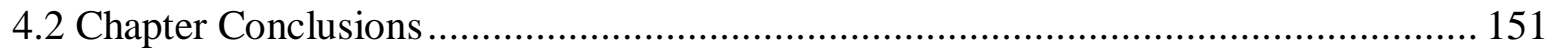

Chapter 5 , Study of the continuous uptake of $\mathrm{Cu}^{2+}$ by NCaSil ..................................... 153

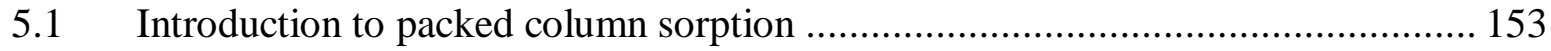

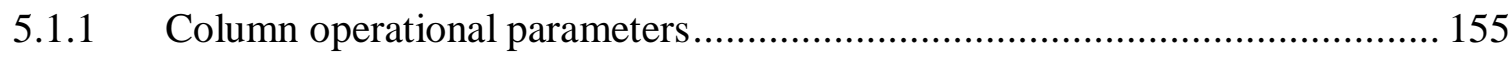

5.1.2 Borrmann and Fonseca approach to the continuous uptake of $\mathrm{Cu}^{2+} \ldots \ldots \ldots \ldots \ldots . . . . . . . .159$

5.2 Results and discussion for the continuous uptake of $\mathrm{Cu}^{2+}$ using NCaSil .................. 162

5.2.1 Continuous uptake of $\mathrm{Cu}^{2+}$ using a conventional axial flow column................... 163

5.2.2 Continuous uptake of $\mathrm{Cu}^{2+}$ onto NCaSil using a radial flow .............................. 176

5.2.3 Continuous uptake of $\mathrm{Cu}^{2+}$ onto NCaSil using a radial flow column immersed in a tank containing a copper sulfate solution........................................................... 182

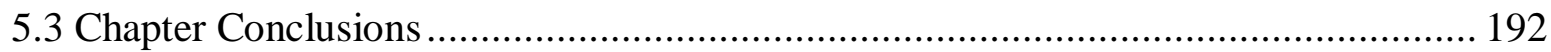

Chapter 6, Thesis Conclusions..................................................................................... 194

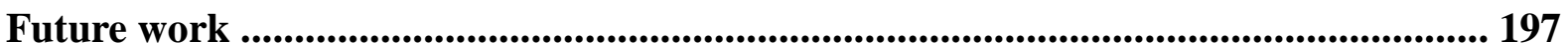

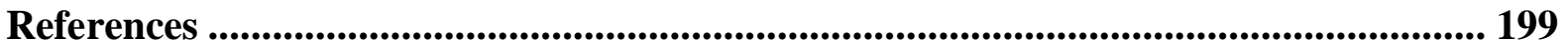

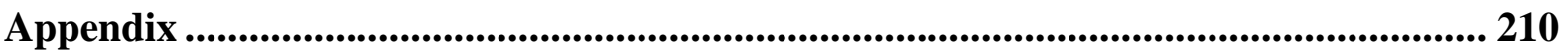




\section{List of Figures}

Figure 1.1: Scanning electron micrograph of the NCaSil surface and platelet structure. [37] .. 9

Figure 1.2: Suggested formula and schematic of nanostructured calcium silicate, showing the chain-like nature of the material [37]. Calcium is present on the surface and in between silicate layers. Dashed lines represent bonds to other silicate chains.

Figure 1.3: $\mathrm{OH}^{-}$and $\mathrm{Ca}^{2+}$ leaching from NCaSil. Graph taken from reference [37]. 11

Figure 2.1: Packed column with NCaSil 25

Figure 2.2: Common arrangement for the studies done with a conventional axial flow column packed with NCaSil. The black arrow on the picture represents the direction of flow. 26

Figure 2.3, Components of the RFC. The column may be used only with the sorbent holder, feed cylinder and inner lid, without the housing and outer components. 26

Figure 2.4: Ratio of the inner feed cylinder diameter to the sorbent holder diameter to the cylinder to the length. The ratio is a:2a:4a. 27

Figure 2.5, Cross-sectional scheme of the continuous sorption device during operation. The setup was the same as the shown in Figure 2.2 for the conventional axial flow column. 28

Figure 2.6: RFC operation by immersion. The figure shows the RFC submerged in a stirred tank containing a $\mathrm{Cu}^{2+}$ solution. The solution is being recirculated by a peristaltic pump...... 29

Figure 2.7: Error contributions for the preparation of a $\mathrm{Cu}^{2+}$ calibration curve. 40

Figure 2.8: Error contribution for kinetic experiments done as described in Section 2.3.2 and 2.3.4.1......

Figure 3.1: General sorption mechanism onto a spherical porous solid. 46

Figure 3.2: Kinetic study with different stirring speeds at 293 K. Experimental conditions adsorbent dosage $1 \mathrm{~g} \mathrm{~L}^{-1} ;\left[\mathrm{Cu}^{2+}\right]_{\text {initial }} 15.7 \mathrm{mmol} \mathrm{L}^{-1}$

Figure 3.3: Experimental and modelled kinetic data for $\mathrm{Cu}^{2+}$ uptake onto NCaSil for temperatures ranging between 277 and $347 \mathrm{~K}$. Experimental conditions: adsorbent dosage $1 \mathrm{~g}$ $\mathrm{L}^{-1} ;\left[\mathrm{Cu}^{2+}\right]_{\text {initial }} 15.7 \mathrm{mmol} \mathrm{L}^{-1}$. 
Figure 3.4: Arrhenius plot using the kinetic rate constant for $\mathrm{Cu}^{2+}$ uptake onto NCaSil shown in Table 3.2.

Figure 3.5: kinetic plot and pseudo-second order model fit for calcium release at 293, 313 and $333 \mathrm{~K}$. Experimental conditions: adsorbent dosage $1 \mathrm{~g} \mathrm{~L}^{-1}$; $\left[\mathrm{Cu}^{2+}\right]_{\text {initial }} 15.7 \mathrm{mmol} \mathrm{L}{ }^{-1}$..... 56

Figure 3.6: Kinetic plot and pseudo second order model fit for the release of monomeric silica from NCaSil at 293, 313 and $333 \mathrm{~K}$. Experimental conditions: adsorbent dosage $1 \mathrm{~g} \mathrm{~L}^{-1}$; $\left[\mathrm{Cu}^{2+}\right]_{\text {initial }} 15.7 \mathrm{mmol} \mathrm{L}^{-1}$

Figure 3.7: Solubility of amorphous silica at different temperatures over a $\mathrm{pH}$ range taken from reference [67].

Figure 3.8: $\mathrm{pH}$ variation over time at $293 \mathrm{~K}$ for different initial concentration of $\mathrm{Cu}^{2+}$. Experimental conditions: adsorbent dosage $1 \mathrm{~g} \mathrm{~L}^{-1}$

Figure 3.9: $\mathrm{pH}$ variation over time at $303 \mathrm{~K}$ with different initial concentration of $\mathrm{Cu}^{2+}$. Experimental conditions: adsorbent dosage $1 \mathrm{~g} \mathrm{~L}^{-1}$

Figure 3.10: $\mathrm{pH}$ variation over time at $313 \mathrm{~K}$ with different initial concentration of $\mathrm{Cu}^{2+}$. Experimental condition: adsorbent dosage $1 \mathrm{~g} \mathrm{~L}^{-1}$.

Figure 3.11: Kinetic study at $293 \mathrm{~K}$ of crystal growth on NCaSil using powder XRD. $\mathrm{G}=$ calcium sulfate dihydrate, $\mathrm{N}=\mathrm{NCaSil}, \mathrm{W}=$ wroewolfeite, $\mathrm{P}=$ posnjakite and $\mathrm{B}=$ brochantite. Experimental conditions: [ $\mathrm{Cu}^{2+}$ ]initial $15.7 \mathrm{mmol} \mathrm{L}{ }^{-1}$; adsorbent dosage $1 \mathrm{~g} \mathrm{~L}^{-1}$; stirring speed $500 \mathrm{rpm}$ 66

Figure 3.12: Examples taken from the literature for the three copper sulfate hydroxide minerals observed in this study.

Figure 3.13: Kinetic study at $313 \mathrm{~K}$ of crystal growth on NCaSil using powder XRD. $\mathrm{G}=$ calcium sulfate dihydrate, $\mathrm{N}=\mathrm{NCaSil}, \mathrm{P}=$ posnjakite and $\mathrm{B}=$ brochantite. Experimental conditions: $\left[\mathrm{Cu}^{2+}\right]$ initial $15.7 \mathrm{mmol} \mathrm{L}{ }^{-1}$; adsorbent dosage $1 \mathrm{~g} \mathrm{~L}^{-1}$; stirring speed $500 \mathrm{rpm} . . .71$

Figure 3.14: Kinetic study at $333 \mathrm{~K}$ of crystal growth on NCaSil using powder XRD. G = calcium sulfate dihydrate, $\mathrm{N}=\mathrm{NCaSil}, \mathrm{P}=$ posnjakite and $\mathrm{B}=$ brochantite. Experimental conditions: $\left[\mathrm{Cu}^{2+}\right]$ initial $15.7 \mathrm{mmol} \mathrm{L}{ }^{-1}$; adsorbent dosage $1 \mathrm{~g} \mathrm{~L}^{-1}$; stirring speed $500 \mathrm{rpm} . . .73$

Figure 3.15: Kinetics of plane growth for the most representative peaks in brochantite at 333 K. 
Figure 3.16: Brochantite crystal unit cell drawn using mercury 3.0 software using data obtained in reference [81].

Figure 3.17: Atomic contribution to each of the different analysed planes of brochantite using data obtained in reference [81]. 78

Figure 3.18: Kinetic study on the growth of plane 420 at 293, 313 and $333 \mathrm{~K}$. The experimental data presented on the ordinate represents the peak area of the reflection at $33.46^{\circ}$ $2 \theta \mathrm{Cu} \mathrm{K}$ in Figure 3.11, Figure 3.13 and Figure 3.14.

Figure 3.19: Arrhenius plot for the growth of plane 420 of Brochantite using kinetic rate constant reported in Table 3.7.

Figure 3.20: Temperature dependence of the time to reach equilibrium for plane 420 . 82

Figure 3.21: SEM micrograph on backscatter mode for $t=1 \mathrm{~min}$. Experimental conditions: $\left[\mathrm{Cu}^{2+}\right]_{\text {initial }} 15.7 \mathrm{mmol} \mathrm{L}{ }^{-1}$; adsorbent dosage $1 \mathrm{~g} \mathrm{~L}^{-1}$; temperature of the reaction $313 \mathrm{~K} \ldots \ldots . .87$

Figure 3.22, SEM micrograph for $t=5 \mathrm{~min}$. Experimental conditions: $\left[\mathrm{Cu}^{2+}\right]_{\text {initial }} 15.7 \mathrm{mmol}$ $\mathrm{L}^{-1}$; adsorbent dosage $1 \mathrm{~g} \mathrm{~L}^{-1}$; temperature of the reaction $313 \mathrm{~K}$.

Figure 3.23: SEM micrograph on backscatter mode for $t=10 \mathrm{~min}$. Experimental conditions: $\left[\mathrm{Cu}^{2+}\right]_{\text {initial }} 15.7 \mathrm{mmol} \mathrm{L}{ }^{-1}$; adsorbent dosage $1 \mathrm{~g} \mathrm{~L}^{-1}$; temperature of the reaction $313 \mathrm{~K}$

Figure 3.24: SEM image and EDS mapping of Figure 3.23. Si and $\mathrm{Cu}$ are shown in red and blue respectively. Experimental conditions: $\left[\mathrm{Cu}^{2+}\right]_{\text {initial }} 15.7 \mathrm{mmol} \mathrm{L}^{-1}$; adsorbent dosage $1 \mathrm{~g} \mathrm{~L}^{-}$ ${ }^{1}$; temperature of the reaction $313 \mathrm{~K}$.

Figure 3.25: SEM micrograph on backscatter mode for $t=15 \mathrm{~min}$. Experimental conditions: $\left[\mathrm{Cu}^{2+}\right]_{\text {initial }} 15.7 \mathrm{mmol} \mathrm{L}^{-1}$; adsorbent dosage $1 \mathrm{~g} \mathrm{~L}^{-1}$; temperature of the reaction $313 \mathrm{~K}$ 91

Figure 3.26: SEM micrograph on backscatter mode for $t=30 \mathrm{~min}$. Experimental conditions: $\left[\mathrm{Cu}^{2+}\right]_{\text {initial }} 15.7 \mathrm{mmol} \mathrm{L}^{-1}$; adsorbent dosage $1 \mathrm{~g} \mathrm{~L}^{-1}$; temperature of the reaction $313 \mathrm{~K} \ldots \ldots . . .92$

Figure 3.27: SEM micrograph on backscatter mode for $t=60$ min. Experimental conditions: $\left[\mathrm{Cu}^{2+}\right]_{\text {initial }} 15.7 \mathrm{mmol} \mathrm{L}^{-1}$; adsorbent dosage $1 \mathrm{~g} \mathrm{~L}^{-1}$; temperature of the reaction $313 \mathrm{~K} \ldots \ldots . .93$

Figure 3.28: SEM micrograph on backscatter mode for $t=120$ min. Experimental conditions: $\left[\mathrm{Cu}^{2+}\right]_{\text {initial }} 15.7 \mathrm{mmol} \mathrm{L}^{-1}$; adsorbent dosage $1 \mathrm{~g} \mathrm{~L}^{-1}$; temperature of the reaction $313 \mathrm{~K} \ldots \ldots . .94$

Figure 3.29: $\mathrm{Cu}^{2+}$ adsorption isotherms obtained using procedure $\mathrm{A}$ for temperatures ranging 293 to 333 K. Experimental conditions are described in section 2.3.2 .............................. 98 
Figure 3.31: Van't Hoff plot using the values of $K_{L}$ shown in Table 3.12 in $\mathrm{L} \mathrm{mol}^{-1}$. 104

Figure 3.32: Isothermal study at $293 \mathrm{~K}$ at the equilibrium of $\mathrm{Cu}^{2+}$ uptake using XRD. $\mathrm{C}=$ calcium carbonate, $\mathrm{W}=$ wroewolfeite; $\mathrm{P}=$ posnjakite and $\mathrm{B}=$ brochantite. Experimental conditions: $\left[\mathrm{Cu}^{2+}\right]_{\text {initial }} 15.7 \mathrm{mmol} \mathrm{L}{ }^{-1}$; adsorbent dosage $1 \mathrm{~g} \mathrm{~L}^{-1}$; stirring speed $500 \mathrm{rpm} . . . .106$

Figure 3.33: Isothermal study at $303 \mathrm{~K}$ at the equilibrium of $\mathrm{Cu}^{2+}$ uptake using XRD. $\mathrm{C}=$ calcium carbonate, $\mathrm{W}=$ wroewolfeite $; \mathrm{P}=$ posnjakite and $\mathrm{B}=$ brochantite. Experimental conditions: $\left[\mathrm{Cu}^{2+}\right]_{\text {initial }} 15.7 \mathrm{mmol} \mathrm{L}{ }^{-1}$; adsorbent dosage $1 \mathrm{~g} \mathrm{~L}^{-1}$; stirring speed $500 \mathrm{rpm} . \ldots .107$

Figure 3.34: Isothermal study at $313 \mathrm{~K}$ at the equilibrium of $\mathrm{Cu}^{2+}$ uptake using $\mathrm{XRD}$. $\mathrm{C}=$ calcium carbonate, $\mathrm{W}=$ wroewolfeite; $\mathrm{P}=$ posnjakite and $\mathrm{B}=$ brochantite. Experimental conditions: $\left[\mathrm{Cu}^{2+}\right]_{\text {initial }} 15.7 \mathrm{mmol} \mathrm{L}{ }^{-1}$; adsorbent dosage $1 \mathrm{~g} \mathrm{~L}^{-1}$; stirring speed $500 \mathrm{rpm} . . . .108$

Figure 3.35: Dependency between $\mathrm{Cu}^{2+}$ uptake and $\mathrm{Ca}^{2+}$ release at the equilibrium. Experimental data was gathered as described in procedure B in section 2.3.2 .................. 110

Figure 3.36: Expansion of zone 1 of Figure 3.35. Experimental data was gathered as described in procedure $\mathrm{B}$ in section 2.3.2.

Figure 3.37: Expansion of zone 2 of Figure 3.35. Experimental data was gathered as described in procedure $\mathrm{B}$ in section 2.3.2.

Figure 3.38: Expansion of zone 3 of Figure 3.35. Experimental data was gathered as described in procedure $\mathrm{B}$ in section 2.3.2. 113

Figure 3.39: Dependency between $\mathrm{Cu}^{2+}$ uptake and monomeric silica release. 114

Figure 3.40: Linear regression to Zone B shown in Figure 3.39.

Figure 3.41: $\mathrm{Cu}^{2+}$ adsorption and its relation to the initial $\mathrm{pH}$ of the solution. Experimental conditions: $\left[\mathrm{Cu}^{2+}\right]_{\text {initial }} 15.7 \mathrm{mmol} \mathrm{L}{ }^{-1}$; adsorbent dosage $1 \mathrm{~g} \mathrm{~L}^{-1}$; $\mathrm{pH}$ adjusted with $\mathrm{H}_{2} \mathrm{SO}_{4} . .116$

Figure 3.42: $\mathrm{Ca}^{2+}$ and monomeric silica release as a function of $\mathrm{pH}$. Experimental conditions: $\left[\mathrm{Cu}^{2+}\right]_{\text {initial }} 0 \mathrm{mmol} \mathrm{L}{ }^{-1}$; adsorbent dosage $1 \mathrm{~g} \mathrm{~L}^{-1}$; $\mathrm{pH}$ adjusted with $\mathrm{H}_{2} \mathrm{SO}_{4}$.

Figure 3.43: $\mathrm{Ca}^{2+}$ and monomeric silica release in the presence of copper at different $\mathrm{pH}$ values. Experimental conditions: $\left[\mathrm{Cu}^{2+}\right]_{\text {initial }} 15.7 \mathrm{mmol} \mathrm{L}^{-1}$; adsorbent dosage $1 \mathrm{~g} \mathrm{~L}^{-1}$; $\mathrm{pH}$ adjusted with $\mathrm{H}_{2} \mathrm{SO}_{4}$ 
Figure 3.44: Simplified reaction mechanism for copper adsorption towards the formation of brochantite. Values of initial adsorption rates are shown for temperature ranging from 293 to $333 \mathrm{~K}$. This scheme represents the summary of the findings in Chapter 3. The stepwise simplified reaction of the studied system over time is presented from left to right. Copper ions form $\mathrm{Cu}(\mathrm{OH})_{2}$ on the surface of NCaSil due to the presence of hydroxide groups. Meanwhile, $\mathrm{NCaSil}$ in water leaches calcium ions, $\mathrm{OH}^{-}$and monomeric silica into solution. Sulfate ions react with calcium ions forming gypsum as observed in XRD patterns in Figure 3.11, Figure 3.13 and Figure 3.14. At the same time as this happens, sulfate nucleates onto $\mathrm{Cu}(\mathrm{OH})_{2}$ rapidly forming wroewolfeite and posnjakite at temperatures below $313 \mathrm{~K}$. For temperatures higher than $333 \mathrm{~K}$ it forms brochantite. Nonetheless at low temperatures the transformation of wroewolfeite and posnjakite into brochantite proved to be slow under the studied conditions.

Figure 4.1: Comparison between metal hydroxides and metal sulfides equilibrium concentration taken from reference [103].

Figure 4.2: XRD pattern for the suspended solids from the weak acid sample. Refer to section 2.4.1 for sample collection.

Figure 4.3: Three different types of structures observed in the suspended solids: a) rectangular rhomboids; b) star shapes; and unlabeled sub-micron particles (bright, white dots and clusters of dots). Refer to section 2.4.1 for sample colletion 138

Figure 4.4: SEM-EDS imaging of the rhomboid type of structures found in the suspended solids. Presence of sulfur is shown in blue, oxygen in red and sodium in green. The overlay of oxygen and sulfur is represented as purple and the overlay of oxygen, sulfur and sodium as white. A backscattered electron image is presented in grayscale in the top left corner for orientation.

Figure 4.5: SEM-EDS imaging of the star shaped type of structures observed in the suspended solids. Presence of calcium is represented in blue, oxygen in red and arsenic in green. The overlay of these three elements is shown in white. A backscattered electron image is presented in grayscale in the top left corner for orientation. 140

Figure 4.6: SEM-EDS imaging of the sub-micron particles. Lead is represented as blue, oxygen as red and sulfur as green. The overlay of oxygen, sulfur and lead mapping is represented in light blue. A backscattered electron image is presented in grayscale in the top left corner for orientation. 
Figure 4.7: Emulated mining waste treatment flow sheet.

Figure 4.8: SEM image of the dirty gypsum produced at stage B in Figure 4.7.

Figure 4.9: Backscattered electron image of the dirty gypsum produced at stage B in Figure 4.7 146

Figure 4.10: EDS map of Figure 4.9 with $\mathrm{Cu}$ (red), S (blue) and $\mathrm{O}$ (green). 146

Figure 4.11: EDS map of Figure 4.9 for As (green) and O (red).

Figure 4.12: XRD pattern corresponding to solid fraction B in Figure 4.7. 148

Figure 4.13: Kinetics of copper uptake by NCaSil from solution A in Figure 4.7

Figure 4.14: XRD pattern corresponding to letter $\mathrm{C}$ in Figure 4.7

Figure 5.1, Progression of the sorption zone through a conventional axial flow packed column taken from reference [114].

Figure 5.2: Borrmann design for the treatment of aqueous solutions containing high concentrations of metal ions. The experiment was carried out by Fonseca. The image is a reprint from reference [116]. The dimensions of the cartridge are: internal diameter $3 \mathrm{~cm}$; external diameter $5 \mathrm{~cm}$; length of the chamber where the paper is placed $9 \mathrm{~cm}$.

Figure 5.3: Continuous uptake of copper carried out by Fonseca using the design shown Figure 5.2. Experimental conditions: $\left[\mathrm{Cu}^{2+}\right] 1.6 \mathrm{mmol} \mathrm{L}^{-1}$ using copper chloride; flow $14.5 \mathrm{ml}$ $\min ^{-1}$; Hydraulic residence time $1.3 \mathrm{~min}$; temperature of the feed solution $303 \mathrm{~K}$. This figure was translated to English from the original version in reference [116]. 160

Figure 5.4: Continuous uptake of copper carried out by Fonseca using the design shown Figure 5.2. Experimental conditions: $\left[\mathrm{Cu}^{2+}\right] 1.6 \mathrm{mmol} \mathrm{L}{ }^{-1}$ using copper chloride; flow $14.5 \mathrm{ml}$ $\min ^{-1}$; Hydraulic residence time $1.3 \mathrm{~min}$; temperature of the feed solution $303 \mathrm{~K}$. This figure was translated to English from the original version in reference [116]. 161

Figure 5.5: Conventional axial flow column experiment for the continuous adsorption of $\mathrm{Cu}^{2+}$ ions onto NCaSil. The graphs present the variation in the concentration of $\mathrm{Cu}^{2+}, \mathrm{Ca}^{2+}$ and monomeric silica as $\mathrm{SiO}_{2}$ in solution over time after it has passed through the column. The change of the $\mathrm{pH}$ over time is also shown. Experimental conditions: $\left[\mathrm{Cu}^{2+}\right] 1.6 \mathrm{mmol} \mathrm{L}{ }^{-1}$; bed

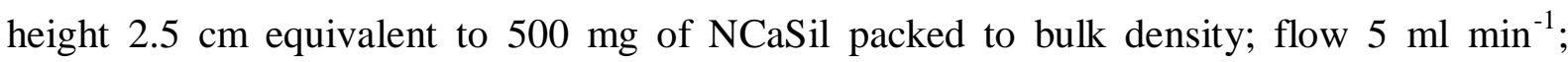
temperature $293 \mathrm{~K}$. Experimental errors are detailed in Table 2.3 
Figure 5.6: Conventional axial flow column experiment for the continuous adsorption of $\mathrm{Cu}^{2+}$ ions onto NCaSil. The graphs present the variation in the concentration of $\mathrm{Cu}^{2+}, \mathrm{Ca}^{2+}$ and monomeric silica as $\mathrm{SiO}_{2}$ in solution over time after it has passed through the column. The change of the $\mathrm{pH}$ over time is also shown. Experimental conditions: $\left[\mathrm{Cu}^{2+}\right] 7.9 \mathrm{mmol} \mathrm{L}{ }^{-1}$; bed height $2.5 \mathrm{~cm}$ equivalent to $500 \mathrm{mg}$ of NCaSil packed to bulk density; flow $5 \mathrm{ml} \mathrm{min}{ }^{-1}$; temperature 293 K. Experimental errors are detailed in Table 2.3

Figure 5.7: Conventional axial flow column experiment for the continuous adsorption of $\mathrm{Cu}^{2+}$ ions onto NCaSil. The graphs present the variation in the concentration of $\mathrm{Cu}^{2+}, \mathrm{Ca}^{2+}$ and monomeric silica as $\mathrm{SiO}_{2}$ in solution over time after it has passed through the column. The change of the $\mathrm{pH}$ over time is also shown. Experimental conditions: $\left[\mathrm{Cu}^{2+}\right] 15.7 \mathrm{mmol} \mathrm{L}{ }^{-1}$;

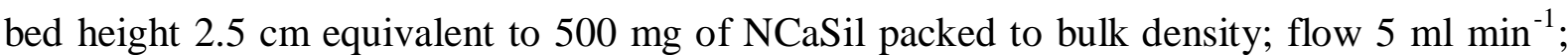
temperature 293 K. Experimental errors are detailed in Table 2.3

Figure 5.8: Conventional axial flow column experiment for the continuous adsorption of $\mathrm{Cu}^{2+}$ ions onto NCaSil. The graphs present the variation in the concentration of $\mathrm{Cu}^{2+}, \mathrm{Ca}^{2+}$ and monomeric silica as $\mathrm{SiO}_{2}$ in solution over time after it has passed through the column. The change of the pressure and the $\mathrm{pH}$ over time is also shown. Experimental conditions: $\left[\mathrm{Cu}^{2+}\right]$ $1.6 \mathrm{mmol} \mathrm{L}{ }^{-1}$; bed height $5 \mathrm{~cm}$ equivalent to $1.00 \mathrm{~g}$ of NCaSil packed to bulk density; flow 5 $\mathrm{ml} \mathrm{min}^{-1}$; temperature $293 \mathrm{~K}$. Experimental errors are detailed in Table 2.3

Figure 5.9: Conventional axial flow column experiment for the continuous adsorption of $\mathrm{Cu}^{2+}$ ions onto NCaSil. The graphs present the variation in the concentration of $\mathrm{Cu}^{2+}, \mathrm{Ca}^{2+}$ and monomeric silica as $\mathrm{SiO}_{2}$ in solution over time after it has passed through the column. The change of the pressure and the $\mathrm{pH}$ over time is also shown. Experimental conditions: $\left[\mathrm{Cu}^{2+}\right]$ $7.9 \mathrm{mmol} \mathrm{L}^{-1}$; bed height $5 \mathrm{~cm}$ equivalent to $1.00 \mathrm{~g}$ of NCaSil packed to bulk density; flow $5 \mathrm{ml} \mathrm{min}{ }^{-1}$; temperature $293 \mathrm{~K}$. Experimental errors are detailed in Table 2.3

Figure 5.10: Conventional axial flow column experiment for the continuous adsorption of $\mathrm{Cu}^{2+}$ ions onto $\mathrm{NCaSil}$. The graphs present the variation in the concentration of $\mathrm{Cu}^{2+}, \mathrm{Ca}^{2+}$ and monomeric silica as $\mathrm{SiO}_{2}$ in solution over time after it has passed through the column. The change of the pressure and the $\mathrm{pH}$ over time is also shown. Experimental conditions: $\left[\mathrm{Cu}^{2+}\right] 15.7 \mathrm{mmol} \mathrm{L}^{-1}$; bed height $5 \mathrm{~cm}$ equivalent to $1.00 \mathrm{~g}$ of NCaSil packed to bulk density; flow $5 \mathrm{ml} \mathrm{min}{ }^{-1}$; temperature $293 \mathrm{~K}$. Experimental errors are detailed in Table 2.3............ 173

Figure 5.11: RFC experiment for the continuous adsorption of $\mathrm{Cu}^{2+}$ ions onto NCaSil. The graphs present the variation in the concentration of $\mathrm{Cu}^{2+}, \mathrm{Ca}^{2+}$ and monomeric silica as $\mathrm{SiO}_{2}$ in solution over time after it has passed through the column. Experimental conditions: 
$\left[\mathrm{Cu}^{2+}\right]_{\text {feed }} 1.6 \mathrm{mmol} \mathrm{L}{ }^{-1}$; logarithmic mean cross sectional area of $7.3 \times 10^{-3} \mathrm{~m}^{2}$; bed height 2 $\mathrm{cm}$; $3.0 \mathrm{~g}$ of NCaSil packed to bulk density; flow $10 \mathrm{ml} \mathrm{min}^{-1}$; temperature $293 \mathrm{~K}$. Experimental errors are detailed in Table 2.3.

Figure 5.12: RFC experiment for the continuous adsorption of $\mathrm{Cu}^{2+}$ ions onto NCaSil. The graphs present the variation in the concentration of $\mathrm{Cu}^{2+}, \mathrm{Ca}^{2+}$ and monomeric silica as $\mathrm{SiO}_{2}$ in solution over time after it has passed through the column. Experimental conditions: $\left[\mathrm{Cu}^{2+}\right]_{\text {feed }} 7.9 \mathrm{mmol} \mathrm{L}{ }^{-1}$; logarithmic mean cross sectional area of $7.3 \times 10^{-3} \mathrm{~m}^{2}$; bed height 2

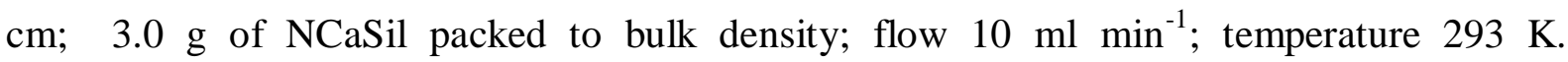
Experimental errors are detailed in Table 2.3.

Figure 5.13: RFC experiment for the continuous adsorption of $\mathrm{Cu}^{2+}$ ions onto NCaSil. The graphs present the variation in the concentration of $\mathrm{Cu}^{2+}, \mathrm{Ca}^{2+}$ and monomeric silica as $\mathrm{SiO}_{2}$ in solution over time after it has passed through the column. Experimental conditions: $\left[\mathrm{Cu}^{2+}\right]_{\text {feed }} 15.7 \mathrm{mmol} \mathrm{L}^{-1}$; logarithmic mean cross sectional area of $7.3 \times 10^{-3} \mathrm{~m}^{2}$; bed height 2

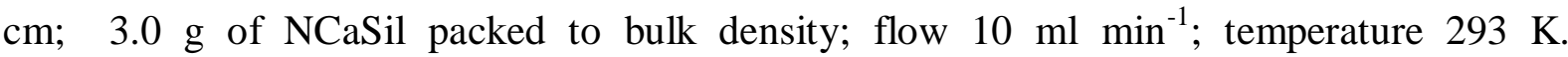
Experimental errors are detailed in Table 2.3.

Figure 5.14: RFC experiment for the continuous adsorption of $\mathrm{Cu}^{2+}$ ions onto NCaSil. The graphs present the variation in the concentration of $\mathrm{Cu}^{2+}, \mathrm{Ca}^{2+}$ and monomeric silica as $\mathrm{SiO}_{2}$ in solution over time after it has passed through the column. The variation of the concentration of $\mathrm{Cu}^{2+}$ over time for the same initial concentration of $\mathrm{Cu}^{2+}$ is also presented.

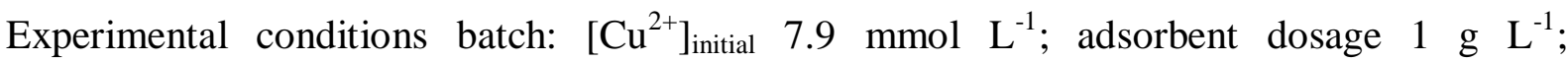
temperature $293 \mathrm{~K}$; stirring speed $500 \mathrm{rpm}$. Experimental conditions RFC: [ $\left.\mathrm{Cu}^{2+}\right]_{\text {feed }} 1.6 \mathrm{mmol}$ $\mathrm{L}^{-1}$; logarithmic mean cross sectional area of $7.3 \times 10^{-3} \mathrm{~m}^{2}$; bed height $2 \mathrm{~cm} ; 3.0 \mathrm{~g}$ of NCaSil packed to bulk density; flow $200 \mathrm{ml} \mathrm{min}^{-1}$; temperature $293 \mathrm{~K}$. Experimental errors are detailed in Table 2.3 .

Figure 5.15: RFC experiment for the continuous adsorption of $\mathrm{Cu}^{2+}$ ions onto NCaSil. The graphs present the variation in the concentration of $\mathrm{Cu}^{2+}, \mathrm{Ca}^{2+}$ and monomeric silica as $\mathrm{SiO}_{2}$ in solution over time after it has passed through the column. The variation of the concentration of $\mathrm{Cu}^{2+}$ over time for the same initial concentration of $\mathrm{Cu}^{2+}$ is also presented. Experimental conditions batch: $\left[\mathrm{Cu}^{2+}\right]_{\text {initial }} 7.9 \mathrm{mmol} \mathrm{L}{ }^{-1}$; adsorbent dosage $1 \mathrm{~g} \mathrm{~L}^{-1}$; temperature $293 \mathrm{~K}$; stirring speed $500 \mathrm{rpm}$. Experimental conditions RFC: $\left[\mathrm{Cu}^{2+}\right]_{\text {feed }} 7.9 \mathrm{mmol}$ $\mathrm{L}^{-1}$; logarithmic mean cross sectional area of $7.3 \times 10^{-3} \mathrm{~m}^{2}$; bed width $2 \mathrm{~cm} ; 3.0 \mathrm{~g}$ of NCaSil packed to bulk density; flow $200 \mathrm{ml} \mathrm{min}^{-1}$; temperature $293 \mathrm{~K}$. Experimental errors are detailed in Table 2.3. 
Figure 5.16: Ratio of the inner feed cylinder diameter to the sorbent holder diameter to the cylinder to the length. The ratio is a:2a:4a. 


\section{List of Tables}

Table 1.1: Discharge limits in New Zealand and Chile

Table 1.2, Summary of $\mathrm{Cu}^{2+}$ sorption onto various sorbents.

Table 1.3: Possible molar mass for NCaSil

Table 2.1: Reagents for the preparation of the weak acid

Table 2.2: preparation of standards for monomeric silica measurements. 32

Table 2.3: Summary of total expanded uncertainty

Table 3.1: Pseudo-second order model kinetic parameters for the uptake of $\mathrm{Cu}^{2+}$ with different stirring speed at $293 \mathrm{~K}$ for the plot presented in Figure 3.2.

Table 3.2: Kinetic parameters for the pseudo-second order equation calculated from the data shown in Figure 3.3.

Table 3.3: Pseudo-second order kinetic parameters for the release of calcium to the solution at 293, 313 and $333 \mathrm{~K}$ calculate from the data presented in Figure 3.5. 56

Table 3.4: Pseudo-second order kinetic parameters for the release of monomeric silica into solution at 293, 313 and $333 \mathrm{~K}$, calculated from experimental data shown in Figure 3.6...... 59

Table 3.5: $\mathrm{pH}$ values at the equilibrium. 64

Table 3.6: kinetic parameters for plane growth in brochantite crystals at $333 \mathrm{~K}$ 76

Table 3.7: Pseudo-first order kinetic parameters for the development of plane 420 in Brochantite. 80

Table 3.8: Mass balance for the uptake of $\mathrm{Cu}^{2+}$ onto NCaSil 84

Table 3.9: Stoichiometric coefficients for Table 3.8 84

Table 3.10: Overall mass balance result 85 
Table 3.11, Langmuir, Freundlich and Temkin isotherms parameters using procedure A described in Section 2.3.2. Constants were obtained using experimental data plotted in Figure 3.29 99

Table 3.12: Langmuir, Freundlich and Temkin adsorption isotherms parameters using procedure B described in Section 2.3.2. Constants were obtained using experimental data plotted in Figure 3.30.

Table 3.13: Thermodynamical parameters for the adsorption of $\mathrm{Cu}^{2+}$ onto $\mathrm{NCaSil}$ 104

Table 4.1: Summary of the presented control chart parameters for the weak acid generated at Plants 1 and 2 at El Teniente.

Table 4.2: Chemical analysis of the liquid portion of the sample.

Table 4.3: Concentration of different metals and metalloids found in the suspended solids of the weak acid waste collected at El Teniente.

Table 5.1: Advantages and disadvantages of operating the radial flow column in a semi-batch manner by immersion inside a tank. 186

Table 5.2: Radial flow column specifications regarding the volume of solution to be treated and the concentration of $\mathrm{Cu}^{2+}$ in solution. 191 


\section{Glossary}

$[\mathbf{C a}]_{\text {Blank, }}$, concentration of calcium in the blank in $\mathrm{mmol} \mathrm{L}^{-1}$.

$[\mathrm{Ca}]_{\mathrm{eq}}$, concentration of calcium in the sample that has been released at the equilibrium in $\mathrm{mmol} \mathrm{L}^{-1}$.

$[\mathbf{C a}]_{t}$, concentration of calcium in the sample that has been released at time $t$ in $\mathrm{mmol} \mathrm{L}^{-1}$.

$[\mathbf{C u}]_{\text {eq }}$, concentration of copper in the sample at the equilibrium in $\mathrm{mmol} \mathrm{L}^{-1}$.

$[\mathbf{C u}]_{\text {feed }}$ is the concentration of $\mathrm{Cu}^{2+}$ in solution being pumped into a column in $\mathrm{mmol} \mathrm{L}^{-1}$.

$[\mathrm{Cu}]_{\mathfrak{t}}$, concentration of $\mathrm{Cu}^{2+}$ in the sample taken at time $t$ in $\mathrm{mmol} \mathrm{L}^{-1}$.

$\left[\mathrm{Cu}^{2+}\right]_{\text {initial }}$, initial concentration of copper in batch experiments in $\mathrm{mmol} \mathrm{L}^{-1}$.

[Si], is the concentration in the sample for $\mathrm{Si}$ in $\mathrm{mmol} \mathrm{L}^{-1}$.

$\boldsymbol{a}$, length of the internal diameter in metres

$\boldsymbol{A}$, time dependency factor of the Arrhenius equation and has the same units as $k$

$A_{\text {cross,sec }}$, is the cross sectional area of a RFC following a $a: 2 a: 4 a$ ratio

Adsorbent dosage, refers to the amount of adsorbent in grams added to a certain volume of solution in L.

$\boldsymbol{A}_{e q}$, integrated peak area in counts. $2 \theta$ at the equilibrium

$\boldsymbol{A}_{\boldsymbol{l} n}$, mean logarithmic area of a cylindrical wall in $\mathrm{m}^{2}$

AMD, Acid Mine Dranaige

$A_{r}(\mathbf{S i})$, relative atomic mass of silicon

ARD, Acid Rock Dranaige

$\boldsymbol{A}_{t}$, integrated peak area in counts. $2 \theta$ at any time $t$

$\boldsymbol{a}_{T}$, Temkin model constant

$\boldsymbol{B}$, brochantite

$\boldsymbol{b}_{\boldsymbol{T}}$, Temkin model constant

C, calcium carbonate

$\boldsymbol{C}_{\boldsymbol{e}}$, concentration in solution at the equilibrium of a certain analyte

$C_{L}$, length of the radial flow column

COD, chemical oxygen demand in $\mathrm{mg} \mathrm{L}^{-1}$

CODELCO, Corporación Nacional del Cobre, Chilean state owned mining company

Dint, diameter of the inner feed cylinder of the radial flow column

$\boldsymbol{D}_{p}$, modified Reynolds number variable related to the particles diameter of the packing material

$D_{S B}$, diameter of the sorbent holder cylinder

$\boldsymbol{E} \boldsymbol{a}$, activation energy 
EDS, energy dispersive spectroscopy

$\boldsymbol{G}$, superficial mass velocity $(G=H \cdot \rho)$

$\boldsymbol{g}_{\boldsymbol{c}}$, gravitational constant to convert from kg-f to Newton

$\boldsymbol{H}$, hydraulic loading

$\boldsymbol{h}, k q_{e}^{2}$ initial rate of adsorption

$\boldsymbol{k}$, kinetic rate constant

$\boldsymbol{k} \boldsymbol{A}_{e q}$, initial adsorption rate for the pseudo first order model for powder-XRD kinetic followup study

$\boldsymbol{k} \boldsymbol{A}_{e q}{ }^{2}$, initial adsorption rate for the pseudo second adsorption model for powder-XRD kinetic follow-up study

$\boldsymbol{K}_{\boldsymbol{F}}$, Freundlich constant

$\boldsymbol{K}_{\boldsymbol{L}}$, Langmuir constant

$\boldsymbol{k} \boldsymbol{q}_{e}$, initial adsorption rate for the pseudo first adsorption model

$\boldsymbol{k} \boldsymbol{q}_{e}{ }^{2}$, initial adsorption rate for the pseudo second adsorption model

$\boldsymbol{L}$, axial length of the packing chamber of the RFC

$\boldsymbol{L A} \boldsymbol{L}$, lower action limit

$\boldsymbol{L} \boldsymbol{W} \boldsymbol{L}$, lower warning limit

$\boldsymbol{m}_{N C a S i l}$, mass of NCaSil added at beginning of the adsorption experiment in $\mathrm{g}$

$\boldsymbol{n}$, exponential

$\boldsymbol{N}$, nanostructure calcium silicate, $\mathrm{NCaSil}$

NCaSil, nanostructured calcium silicate

$N_{R e}$, modified Reynolds number

$\boldsymbol{P}$, posnjakite

$Q$, flow of a certain fluid

$\boldsymbol{q}_{e}$, amount adsorbed at the equilibrium in $\mathrm{mmol} \mathrm{g}^{-1}$.

$\boldsymbol{q}_{\boldsymbol{m}}$, maximum adsorption capacity or maximum loading capacity of a given material in mmol

$\mathrm{g}^{-1}$. This parameter is obtained from the Langmuir model.

$\boldsymbol{q}_{\boldsymbol{r}, e, \mathrm{Ca}}$ concentration of $\mathrm{Ca}^{2+}$ released at the equilibrium in $\mathrm{mmol} \mathrm{L}^{-1}$

$\boldsymbol{q}_{r, e, S i O 2}$ is the concentration of monomeric silica at the equilibrium expressed as $\mathrm{SiO}_{2}$ in $\mathrm{mmol}$ $\mathrm{L}^{-1}$.

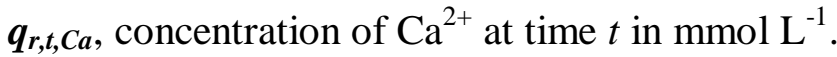

$\boldsymbol{q}_{\boldsymbol{r}, \boldsymbol{t}, \mathrm{SiO} 2}$, concentration of $\mathrm{SiO}_{2}$ at time $t$ in $\mathrm{mmol} \mathrm{L}^{-1}$.

$\boldsymbol{q}_{t}$ is the amount adsorbed of any given adsorbate at time $t$ in $\mathrm{mmol} \mathrm{g}^{-1}$.

$\boldsymbol{R}$, gas constant $8.314 \mathrm{~J} \mathrm{~mol}^{-1} \mathrm{~K}^{-1}$

$\boldsymbol{R e}$, Reynolds number

rpm, Revolution per minute 
$S_{0}$, surface area of particle per unit of volume

SEM, scanning electron microscopy

T, temperature

$\boldsymbol{t}$, time

$\boldsymbol{U}_{\boldsymbol{0}}$, superficial velocity

$\boldsymbol{U A L}$, upper action limit

$\boldsymbol{U W L}$, upper warning limit

$\boldsymbol{v}$, average axial velocity of a fluid

$\boldsymbol{V}_{\text {feed }}$ is the volume of copper sulfate solution utilized in the experiment in L.

$\boldsymbol{V}_{\boldsymbol{R} F \boldsymbol{C}}$, the volume where the NCaSil is packed in the RFC (packing chamber)

$V_{\text {treated }}$, volume to be treated by the RFC column

W, wroewolfeite

XRD, X-ray diffraction

A, integrated peak area of a certain reflection in a powder-XRD pattern in counts. $2 \theta$

$\Delta G$, Gibbs free energy

$\Delta \boldsymbol{H}^{\circ}$, standard enthalpy

$\Delta p$, pressure drop through a packed column

$\Delta S^{\circ}$, standard entropy

$\varepsilon$, void fraction of a packed column from 0 to 1

$\boldsymbol{\mu}$, fluid viscosity in $\mathrm{Pa} \cdot \mathrm{s}$

$\boldsymbol{\rho}$, density of the fluid $\mathrm{kg} \mathrm{m}^{-3}$

$\boldsymbol{\rho}_{N \text { CaSil }}$, bulk density of NCaSil $70 \mathrm{~kg} \mathrm{~m}^{-3}$ 


\section{Chapter 1, Introduction}

Heavy metals in solution and their risk to the environment are well known [1]. Therefore, great effort has been placed in searching for new technologies and the improvement of the actual treating methods. Special emphasis has been placed into the field of adsorbents looking for materials that gather not only exceptional contaminant removal efficiency but are environmentally friendly [2-5]. The motivations have been on one hand, the remediation of contaminated water and on the other hand, the recovery of valuable metal ions.

In this thesis both visions will be addressed through the enrichment of $\mathrm{Cu}^{2+}$ on the surface of a nanostructured calcium silicate a novel adsorbent material patented by Victoria University of Wellington.

\subsection{Background information on New Zealand and Chile water availability and condition}

Water covers three quarters of earth's surface [6], around $94 \%$ of it makes up the oceans and the remainder $6 \%$ is fresh water. Out of the total fresh water $72 \%$ is found in groundwaters and the remainder $27 \%$ in glaciers and ice caps [7]. Most of this comparatively pure water has to be treated physically and chemically in order to achieve drinking water standards. New Zealand possesses large reserves of water accompanied with high rainfall in most places, making the water suitable for human use without major treatment. But not all countries have such abundance, for example, Chile shares New Zealand's situation in the south but in the far north the landscape is covered by desert with an annual rainfall of less than $5.7 \mathrm{~mm}$ [8] making water a scarce resource. The availability of water is crucial to the northern regions of Chile since most of the large scale mines are located in that area. Mining companies 
usually coexist with farmers withdrawing water resources, using water and discharging it back into rivers. Special caution has to be taken since industrial and human activity turns water into a carrier for a wide range of pollutants that vary from organic substances and microbes to metal ions among many others. If these wastewaters are not treated before they are discharged they can deeply affect the environment transporting polluting agents far away from the source.

Metal ions are considered to be harmful if their concentration is higher than allowed limits, since they undergo different chemical reactions some of which mediate the formation of free radicals causing changes to DNA structure [9]. Metals such as iron, copper, chromium, vanadium or cobalt mainly undergo redox-cycling reactions, for other metals like mercury, nickel and cadmium the predominant mechanism consists in bond formation with sulfhydryl groups of proteins and depletion of glutathione [9]. As an example mercury can be bioaccumulated as methyl mercury by fish and subsequently transferred to humans by its consumption [10].

Copper is a cofactor for proteins involved in bioreactions such as respiration, connective tissue formation, iron metabolism, free radical eradication and neurological function [9]. The recommended daily copper intake in adults is $0.9 \mathrm{mg} \mathrm{d}^{-1}$ [11]. In human cells it is found chemically-bonded to proteins through histidine, methionine and cysteine. Recent studies show that the amount of free-copper is less than a single ion per cell, which suggests that it is easily complexed $[9,12]$. There are two main diseases related to copper accumulation in the human tissue. The first one is Menkes disease, which involves the abnormal accumulation of copper in kidneys and intestinal mucosa leading to a deficiency of this element in other systems not allowing copper to participate as a co-factor in proteins. The second is known as Wilson's disease which involves an abnormal copper accumulation in the liver and the brain which may lead to portal hypertension, esophageal varices, chronic active hepatitis, liver cancer, frontal lobe disorder, depression, anxiety and psychosis. In the cell, $\mathrm{Cu}^{2+}$ may be reduced to $\mathrm{Cu}^{+}$ by glutathione and ascorbic acid which may generate free radicals via Fenton chemistry [9]. Dikalov et al. [13] reported that neurotoxic forms of Amyloid $\beta, A \beta$ (1-42), $A \beta$ (1-40), and also $A \beta$ (25-35) stimulated copper-mediated oxidation of ascorbate, whereas nontoxic A $\beta$ (40-1) did not. Dikalov concluded that toxic $A \beta$ peptides stimulate copper mediated oxidation of ascorbate and generation of hydroxyl radicals via the Fenton reaction. Therefore cupric-amyloid peptide may be involved in the pathogenesis of Alzheimer's disease. 
Governments have put special emphasis on reducing the wastes to minimize human exposure and damage to the environment. Maximum discharge limits have been set and are monitored and regulated by different entities and laws.

The allowed concentration in wastewaters in Chile is regulated nationwide by Decreto Supremo $n^{\circ} 90$ [14] and in New Zealand by bylaws specific for each city council respectively. The maximum discharge values in each country for some inorganic contaminants are listed in Table 1.1. 
Table 1.1: Discharge limits in New Zealand and Chile

\begin{tabular}{|c|c|c|}
\hline \multirow[b]{2}{*}{ Element } & Chile* & New Zealand** \\
\hline & \multicolumn{2}{|c|}{$\begin{array}{c}\text { Maximum allowed concentration } \\
{\left[\mathrm{mg} \mathrm{L}^{-1}\right]}\end{array}$} \\
\hline $\mathrm{Al}$ & 1 & 300 \\
\hline As & 0.5 & 5 \\
\hline $\mathrm{Cd}$ & 0.01 & 0.5 \\
\hline $\mathrm{Cu}$ & 1 & 10 \\
\hline Cr total & 0.1 & \\
\hline $\mathrm{Cr}(\mathrm{VI})$ & 0.05 & \\
\hline Sn & 0.5 & 20 \\
\hline $\mathrm{Fe}$ & 5 & 300 \\
\hline $\mathrm{Mn}$ & 0.3 & 20 \\
\hline $\mathrm{Hg}$ & 0.001 & 0.005 \\
\hline Mo & 1 & 10 \\
\hline $\mathrm{Ni}$ & 3 & 10 \\
\hline $\mathrm{Pb}$ & 0.5 & 10 \\
\hline $\mathrm{Se}$ & 0.1 & 10 \\
\hline $\mathrm{Sb}$ & - & 10 \\
\hline $\mathrm{Zn}$ & 20 & 10 \\
\hline
\end{tabular}

*Discharge limits in Chile are for discharges into rivers.

**Discharge limits correspond to the trade waste bylaw of Wellington City Council, chosen as an example. [15]

The summarized information helps as an overview of the regulations and they are not meant to be compared since in New Zealand waste water enters a collection system that has the outlet in the water treatment plant of the region.

Limits set in Chile help as a guideline towards the concentrations that has to be met in a process that intends to clean waste waters prior to discharge into a body of water. 


\subsection{Adsorbents}

An adsorbent could be defined as a material that possesses available sites on its surface with which molecules/atoms can interact or react. The ideal adsorbent can be defined as one that has the following properties: high loading capacity; fast kinetics; in the presence of the target adsorbate the equilibrium is highly-displaced towards the formation of products; effective regeneration and recovery of the adsorbed species; high surface area; pore size that allows intraparticle diffusion of the targeted atom, ion or molecule; a mean particle size that allows continuous column operations without large backpressures; adsorbs the contaminant of interest at a wide range of $\mathrm{pH}$; and, selectivity towards the adsorbate of interest in presence of other potential adsorbates. Furthermore, other properties could be desired such as low cost and being environmentally benign. In the literature a wide range of adsorbents have been proposed and studied for the uptake of metals from solutions.

Usually adsorbents are able to remove target molecules or ions in the range of millimoles of molecules, atoms or ions per gram of adsorbent. In the case of ion adsorption from solution these adsorptions are in the order of 0.05 to $10 \mathrm{mmol} \mathrm{g}^{-1}$ $[4,5,16-30]$, being most commonly 0.1 to $1 \mathrm{mmol} \mathrm{g}^{-1}$. One could consider as commercially competitive an adsorbent which can remove from solution $1 \mathrm{mmol}$ of a certain ion or molecule per gram of adsorbent used.

Recently different low cost sorbents mainly biomass from by-products of industrial production or simply "useless" biomass (e.g sawdust, rice husk), have been studied as sorbents for metal ions in solution [22, 29, 31]. Biomass certainly has been seen to be a successful sorbent but major drawbacks come from the biodegradation of the structure which limits its use in long term applications [24]. Ion exchange resins and materials have also been studied and applied for the removal of metal ions [26, 32, 33] since they offer the opportunity of regenerating the adsorptive properties. Typically the main difference between adsorbents is related to their surface chemistry. For example, biomass adsorbents have organic functional groups such as amino, alcohol and carboxylates on the surface. On the other hand, some adsorbents may offer exchangeable ions on the surfaces such as ion exchange resin (IX) or zeolites. These materials adsorb the target ion by releasing $\mathrm{H}^{+}, \mathrm{OH}^{-}, \mathrm{Cl}^{-}, \mathrm{Na}^{+}$or $\mathrm{NH}_{4}{ }^{+}$, depending whether an anion or cation is being exchanged. The above mentioned groups offer to immobilize a molecule, atom or ion on the surface by strong interactions that range from the electrostatic (ion exchange) to the formation 
of covalent bonds (organic functional groups). Other adsorbents may rely on weak interactions such as van der waals forces, dipole to dipole interaction or even hydrophobicity to uptake molecules.

In the literature many adsorbents are tested against solutions containing $\mathrm{Cu}^{2+}$ as this ion is a relatively safe and cheap example to study for metal ion sorption. In Table 1.2 are shown the adsorption capacity and the Langmuir constant (equilibrium constant) found in the literature for copper sorption onto various sorbents. 
Table 1.2, Summary of $\mathrm{Cu}^{2+}$ sorption onto various sorbents.

\begin{tabular}{|c|c|c|c|}
\hline Material & $\begin{array}{c}q_{m} \\
{\left[\mathrm{mmol} \mathrm{g}^{-1}\right]}\end{array}$ & $\begin{array}{c}K_{L} \\
{\left[\mathrm{~L} \mathrm{mmol}^{-1}\right]}\end{array}$ & Reference \\
\hline
\end{tabular}

\section{Biosorbent}

Chitosan $0.08-3.5 \quad-$

Crosslinked chitosan $\quad 0.79-1.26$

Saw dust $\quad 0.22$

$\begin{array}{lll}\text { Rice bran } & 0.53 & 14.9\end{array}$

[29]

Zeolites

\begin{tabular}{lc}
\hline Cliptonolite & $0.03-0.06$ \\
Chabacite & 0.08 \\
Chabazite-phillipsite & 0.006 \\
Clay &
\end{tabular}

\begin{tabular}{lccc}
\hline Fly ash-wollostonite & 0.019 & - & [28] \\
Dolomite (turkish) & 0.13 & 4445 &
\end{tabular}

Ion Exchange Resins (IX)

\begin{tabular}{llll}
\hline Dowex 50 & 0.35 & 0.953 & [26] \\
Lewatit CNP 80 & 0.16 & 0.445 & [27]
\end{tabular}

Other

\begin{tabular}{lcc}
\hline Iron III waste fertilizer industry & 0.25 & - \\
Activated carbon & $0.14-0.76$ & - \\
$\begin{array}{l}\text { Chitosan-coated } \\
\text { nanoparticles modified with } \alpha-\end{array}$ & 1.51 & 3.13 \\
ketoglutaric acid & &
\end{tabular}

From the data given in Table 1.2 it can be seen that generally biosorbents present a larger loading capacity of copper ions than other materials. This can be explained by the large number of functional groups on their surface that can interact with the metal ions. Most of the adsorbents have a mean Langmuir constant ${ }^{1}$ value of 0.1 $1 \mathrm{~L} \mathrm{mmol}^{-1}$ but in the case of turkish-dolomite the value is two orders of magnitude

\footnotetext{
1 The Langmuir constant is the equilibrium constant of an adsorption process which exhibits a Langmuir behaviour. For more information please refer to section 3.3.1.
} 
higher. This difference may be attributed to the difference in the type of reaction with the surface.

\subsection{Nanostructured Calcium Silicate Hydrate (NCaSil)}

NCaSil is a proprietary material reported by Johnston, McFarlane and Borrmann [35]. The synthesis is achieved via precipitation of dissolved silica by calcium ions at alkaline $\mathrm{pH}$ under controlled conditions of mixing, temperature and ageing time. The properties of NCaSil materials were studied extensively by McFarlane [36]. NCaSil consists of nanometre platelets stacked semi-randomly, forming aggregated particles of a few micrometres in size (approx. 1-100 $\mu \mathrm{m}$ ). The length of such structure is proportional to the ageing time. The macrostructure is similar in shape to a gypsum desert rose but additional information from X-ray powder diffraction (XRD) shows no long range order thus indicating that the material is amorphous or polycrystalline. Moreover, only two broad peaks arise from XRD analysis which further indicates a two dimensional ordering within the material commonly seen in calcium silicate hydrates. Surface analysis revealed that NCaSil has a high surface area, typically in the order of $300-700 \mathrm{~m}^{2} \mathrm{~g}^{-1}$. A micrograph of the NCaSil surface and platelet structure is shown in Figure 1.1. 


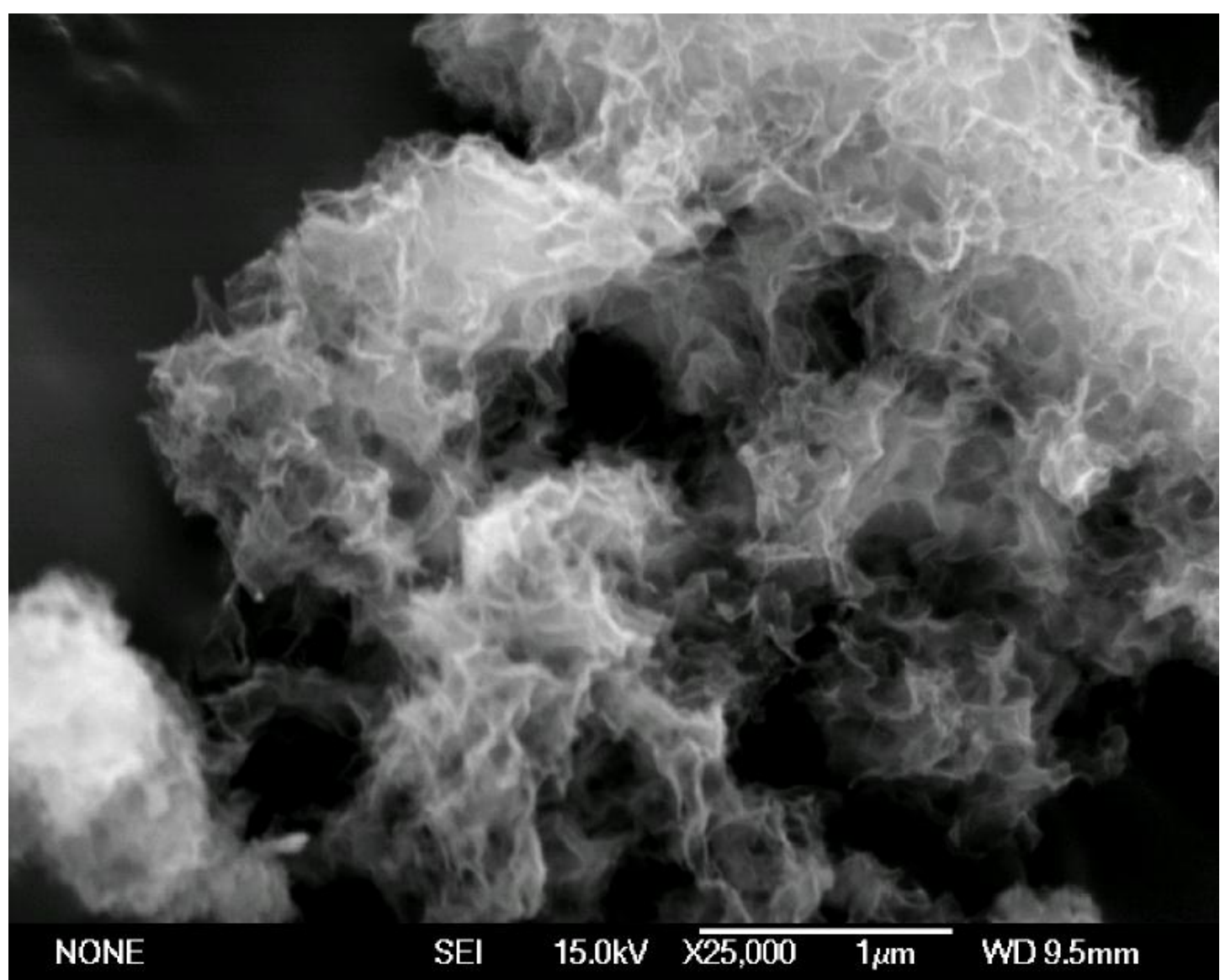

Figure 1.1: Scanning electron micrograph of the NCaSil surface and platelet structure. [37]

The structure of the $\mathrm{NCaSil}$ is believed to consist of tetrahedral $\mathrm{SiO}_{4}{ }^{4-}$ connected through chains bridged by $\mathrm{Ca}^{2+}$ ions as shown in Figure 1.2. The material is mainly composed by $\mathrm{Q}^{2} \mathrm{Si}$ with few $\mathrm{Q}^{1}, \mathrm{Q}^{3}$ and $\mathrm{Q}^{4}$ as reflected from ${ }^{29} \mathrm{Si}-\mathrm{MAS}-\mathrm{NMR}$ reported by McFarlane [36], suggesting long chains of tetrahedral silicate.

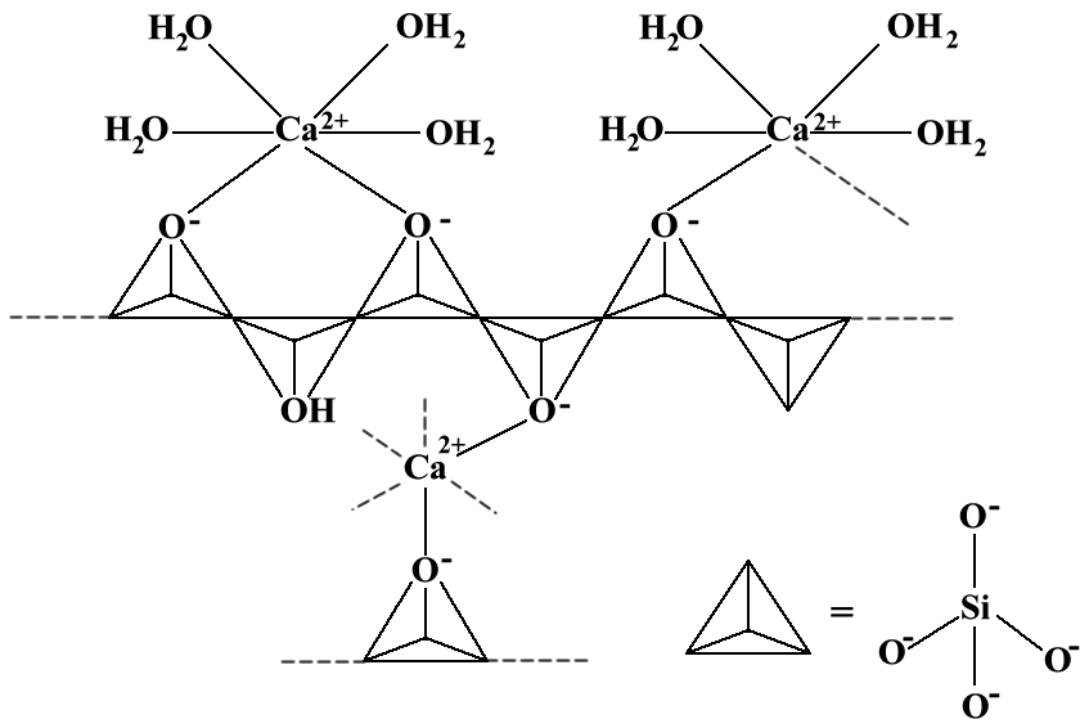

Figure 1.2: Suggested formula and schematic of nanostructured calcium silicate, showing the chain-like nature of the material [37]. Calcium is present on the surface and in between silicate layers. Dashed lines represent bonds to other silicate chains. 
Several spectroscopic techniques such as solid state ${ }^{1} \mathrm{H}-\mathrm{MAS}-\mathrm{NMR}$, X-ray photoelectron spectroscopy (XPS), X-ray fluorescence (XRF) and ion-beam spectroscopic studies of the material have demonstrated the presence of physisorbed water and surface-bound hydroxyl groups. The results led to the empirical formula,

$\mathrm{Ca}_{w} \mathrm{SiO}_{x}(\mathrm{OH})_{y} \cdot z \mathrm{H}_{2} \mathrm{O}$

where: $\mathrm{w}=0.05-1, \mathrm{x}=2.05-3, \mathrm{y}=0-0.5, \mathrm{z}=1.4-2.5$.

Although a value of w equal to 0.05 is unlikely as this is obtained when the NCaSil is contacted with a low $\mathrm{pH}$ solution to create $\mathrm{pH}$-adjusted $\mathrm{NCaSil}$, thus a range of 0.5 - 1 was taken into consideration. Taking in consideration these stoichiometric values reported by McFarlane [36] we may calculate an approximate molar mass for NCaSil in three ranges of content. These values are shown in Table 1.3.

Table 1.3: Possible molar mass for NCaSil

\begin{tabular}{|c|c|c|c|c|}
\hline Low-range & $\mathbf{w}$ & $\mathbf{x}$ & $\mathbf{y}$ & $\mathbf{z}$ \\
\hline & 0.5 & 2.05 & 0 & 1.4 \\
\hline & \multicolumn{4}{|c|}{ NCaSil Molar mass $\left[\mathrm{g} \mathrm{mol}^{-1}\right.$ ] } \\
\hline & \multicolumn{4}{|c|}{106.12} \\
\hline \multirow[t]{4}{*}{ Mid-range } & $\mathbf{w}$ & $\mathbf{x}$ & $\mathbf{y}$ & $\mathbf{z}$ \\
\hline & 0.75 & 2.53 & 0.25 & 1.9 \\
\hline & \multicolumn{4}{|c|}{ NCaSil Molar mass $\left[\mathrm{g} \mathrm{mol}^{-1}\right.$ ] } \\
\hline & \multicolumn{4}{|c|}{137.07} \\
\hline \multirow[t]{4}{*}{ High-range } & $\mathbf{w}$ & $\mathbf{x}$ & $\mathbf{y}$ & $\mathbf{z}$ \\
\hline & 1 & 3 & 0.5 & 2.5 \\
\hline & \multicolumn{4}{|c|}{ NCaSil Molar mass $\left[\mathrm{g} \mathrm{mol}^{-1}\right.$ ] } \\
\hline & \multicolumn{4}{|c|}{169.66} \\
\hline
\end{tabular}

*Compositions are not electrically neutral but serve as an approximation for NCaSil true molar mass

The values shown in Table 1.3 are an approximation to the real molar mass since different combinations of the stoichiometric coefficients can be taken resulting in a molar mass within those boundaries. In this thesis the mid-range value will be used for calculations.

It is believed that the material structure relates to wollastonite $\left(\mathrm{CaSiO}_{3}\right)$ due to a phase transformation of $\mathrm{NCaSil}$ into this mineral at temperatures higher than $720^{\circ} \mathrm{C}$.

The fabrication of NCaSil presents challenges during the drying step, loosing water by evaporation due to the large surface area that creates a high surface tension. As the material is dried water begins to evaporate causing the platelet structure to 
collapse. The loss of the microstructure reduces the surface area of the material. Three different approaches have been developed by McFarlane et al. [36] to overcome this collapse.

1) During the filtration step replace the solvent (water) within the microstructure of NCaSil by one with a lower surface tension, such as ethanol.

2) Reinforcement of the NCaSil structure by condensing a layer of silica between the adjacent platelets of the material. The reinforcement is achieved by contacting a slurry of NCaSil with a solution of silicate, where $\mathrm{H}_{(4-n)} \mathrm{SiO}_{4 \mathrm{n}}{ }^{-}$ions will preferably be sorbed in the acute angle of the platelets reducing the surface tension and increasing the stability of the system. Subsequent acidification will cause the silicate ions to form a silica layer. This material can be wetted and dried several times, without causing significant reduction of its surface area.

3) Acid treatment of the NCaSil slurry: calcium ions are exchanged by protons generating new silanol groups. These new silanol groups can condense reducing the surface tension. This modified NCaSil can be wetted and dried several times just like a reinforced one. However, this method is wasteful of reagents, as most of the calcium is leached out.

When contacted with water $\mathrm{NCaSil}$ releases $\mathrm{OH}^{-}$and $\mathrm{Ca}^{2+}$ ions until equilibrium is reached. In Figure 1.3 is shown the leaching of $\mathrm{OH}^{-}$and $\mathrm{Ca}^{2+}$ ions from $\mathrm{NCaSil}$ as a function of time when contacted with a $\mathrm{pH} 7.2$ solution (water).

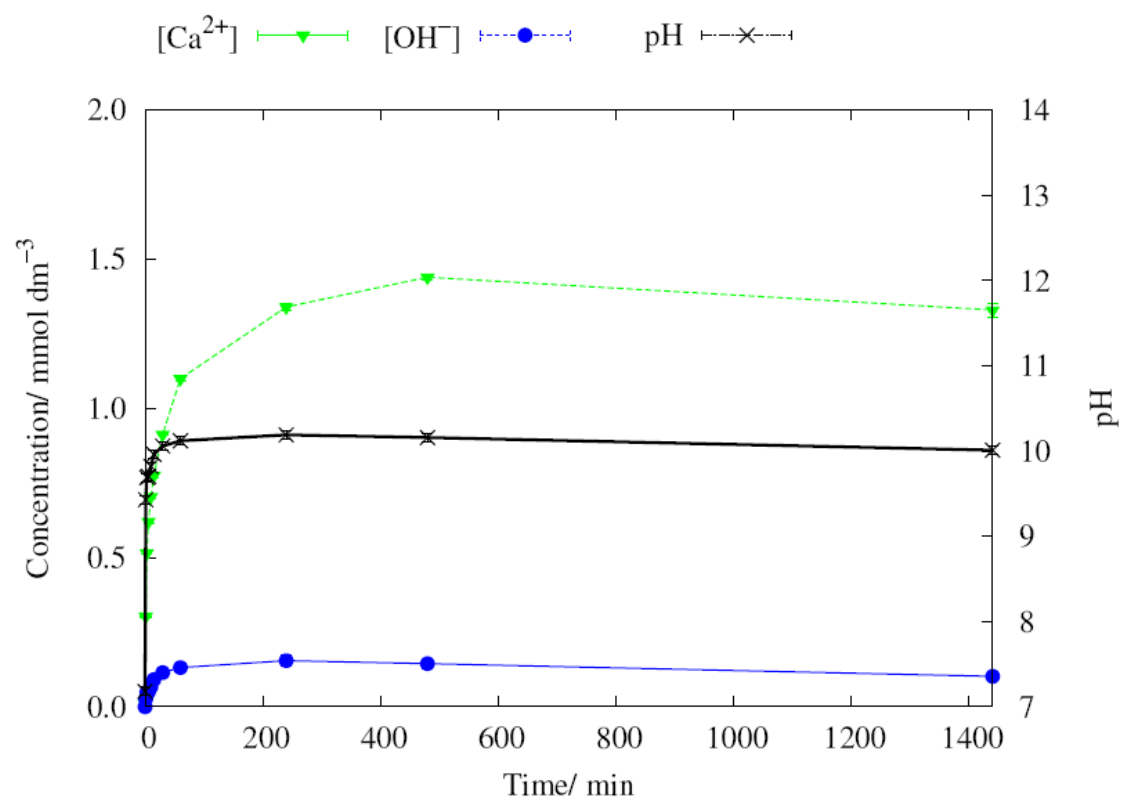

Figure 1.3: $\mathrm{OH}^{-}$and $\mathrm{Ca}^{2+}$ leaching from NCaSil. Graph taken from reference [37]. 
Cairns [37] proposed a two step mechanism for the release of calcium and hydroxide ions into the solution with the NCaSil attaining a negative charge.

$$
\begin{aligned}
& (\mathrm{CaO})_{0.8} \mathrm{SiO}_{2} \rightleftharpoons(\mathrm{CaO})_{0.8} \mathrm{SiO}_{2} \mathrm{Ca}^{2+}+(\mathrm{CaO})_{(0.8-x)} \mathrm{SiO}_{(2+x)}^{2 x-} \\
& (\mathrm{CaO})_{(0.8-x)} \mathrm{SiO}_{(2+x)}^{2 x-}+2 x \mathrm{H}_{2} \mathrm{O} \rightleftharpoons(\mathrm{CaO})_{(0.8-x)}(\mathrm{OH})_{2 x} \mathrm{SiO}_{(2-x)}+2 x \mathrm{OH}^{-}
\end{aligned}
$$

Hydroxide ions react further with the silicate backbone to release monomeric silica into the solution.

$$
\begin{aligned}
& (\mathrm{CaO})_{(0.8-x)} \mathrm{SiO}_{2}(\mathrm{OH})_{x}+(4-x) \mathrm{OH}^{-} \rightleftharpoons \\
& (\mathrm{CaO})_{(0.8-x)} \mathrm{Si}_{(1-x)} \mathrm{O}_{2}^{(4-x)-}+\mathrm{Si}_{x}(\mathrm{OH})_{4}
\end{aligned}
$$

For most silicates the chemical reaction given in eq. 1.4 occurs over long periods of days to weeks and is unlikely that it contributes to the overall process giving rise to a discrepancy with the experimental results [37]. Nevertheless the mechanism depicts well the leaching of ions when the solution has a low $\mathrm{pH}$ value and/or contains a large amount of exchangeable metal ions. However, the microstructure of the material is lost (time $<10 \mathrm{~min}$ ) forming a soluble form of silicate when $1 \mathrm{~g}$ of NCaSil is contacted with $1 \mathrm{~L}$ of a pH 1 solution. Therefore, studies have been limited to $\mathrm{pH}$ values $>4$. The sorption of various cations and anions has been studied, taking into consideration the possibility of ion exchange and formation of insoluble compounds by the presence of $\mathrm{OH}^{-}$and $\mathrm{Ca}^{2+}$ in the structure. Borrmann [38] reported the formation of a Ca-I complex when molecular iodine was adsorbed. Intensive research has been carried out by Southam [39] on phosphate uptake with NCaSil by the precipitation of insoluble calcium phosphates species on the surface.

Cairns et al. [40] studied the sorption of $\mathrm{CuNO}_{3}$ and $\mathrm{CuCl}_{2}$ at different concentrations showing that crystals of rouatite and atacamite were formed readily when concentrations higher than $8.0 \mathrm{mmol} \mathrm{L}^{-1}$ of $\mathrm{Cu}^{2+}$ were used. Furthermore, NCaSil reported to gradually release hydroxide ions into solution when contacted with distilled water. This effect was enhanced when the experiment was carried out using an $80 \mathrm{mmol} \mathrm{L}^{-1} \mathrm{Cu}^{2+}$ solution. Overall, the material exhibited a high loading capacity and fast rate of adsorption with extractions efficiencies of over $95 \%$ for all the different studied salts at concentrations up to $7.9 \mathrm{mmol} \mathrm{L}^{-1}$. Although enough kinetic information for STP conditions was obtained, information about kinetic parameters at different temperatures was not obtained. This is important for the understanding of 
the adsorption mechanism, as well to see if increasing temperature improves or decreases the adsorption of $\mathrm{Cu}^{2+}$. Furthermore, no study over the release of calcium and monomeric silica into the solution at different temperatures was done.

Formation of hydroxide precipitates over clay minerals and metal oxides is not new and it has been reported by several authors for metals such as $\mathrm{Co}^{2+}, \mathrm{Cu}^{2+}$ and $\mathrm{Ni}^{2+}$ [41-43]. In cases where the adsorbent was aluminum-free talc and amorphous silica the adsorption of $\mathrm{Ni}$ resulted in the formation of $\alpha-\mathrm{Ni}(\mathrm{OH})_{2}$-like precipitates [43]. This could be an indication of the first step on the adsorption of copper ions onto the NCaSil forming copper hydroxides on the surface. In fact, Spark et al. [44] showed that copper hydroxide is an intermediate in the formation of copper hydroxide nitrate and copper hydroxide chloride minerals.

The author believes that the mechanism by which the $\mathrm{Cu}^{2+}$ is adsorbed may involve several steps of mass transport towards surface of NCaSil, hydrolysis of the material, ion exchange with calcium ions, precipitation of $\mathrm{Cu}^{2+}$ hydroxide salts and nucleation of $\mathrm{Cu}^{2+}$ onto crystalline structures. In addition, the electrostatic attraction between $\mathrm{Cu}^{2+}$ and the negative surface charge of the silicate should be considered as it was proven by Klimsa [45] using zeta potential measurements over time during the uptake reaction occurred. Moreover, there is also the chance that the release of $\mathrm{Ca}^{2+}$ and $\mathrm{Si}(\mathrm{OH})_{4}$, is independent from the adsorption of $\mathrm{Cu}^{2+}$.

In addition, the ability of $\mathrm{NCaSil}$ to readily uptake copper from solution represents a great potential to treat waste solutions containing a high concentration of copper $\left[\mathrm{Cu}^{2+}\right]$ from $10^{-1}$ to $10 \mathrm{mmol} \mathrm{L}^{-1}$. This kind of waste streams is usually found in the mining industry or naturally occurring as Acid Mine Drainage (AMD). Therefore, the removal of copper from solution will be of great importance not only from an environmental point of view but also under an economic perspective generating a solid with commercial value where the metal ion has been enriched.

Cairns carried out all his studies using $\mathrm{CuNO}_{3} \cdot 3 \mathrm{H}_{2} \mathrm{O}$ and $\mathrm{CuCl}_{2} \cdot 2 \mathrm{H}_{2} \mathrm{O}$. In this work kinetic and equilibrium studies were done utilizing $\mathrm{CuSO}_{4} \cdot 5 \mathrm{H}_{2} \mathrm{O}$ since sulfate concentrations in liquid mining waste are usually higher than nitrate and chloride $[46,47]$.

Fast kinetics and a large maximum loading capacity offers the opportunity to build a device for the continuous adsorption of $\mathrm{Cu}^{2+}$ which can compete with existing materials and processes. Furthermore, the surface chemistry of NCaSil allows the possibility of treating mining waste containing high concentrations of $\mathrm{Cu}^{2+}$ in 
solution resulting in a clean effluent and a copper-rich solid. Regardless of the loss of the microstructure, the formation of copper hydroxide species opens up the possibility of reintroducing this by-product at some stage during the production of copper reducing the costs. 


\subsection{Objectives}

\section{Main objective}

Study the adsorption of $\mathrm{Cu}^{2+}$ ions onto NCaSil in batch and in a continuous setup in order to design a water purification cartridge for high dissolved metal concentration stream decontamination.

\section{Secondary objectives}

- Identify the steps in the mechanism by which $\mathrm{Cu}^{2+}$ is adsorbed onto the surface of NCaSil. Establish rates of the different processes occurring during the uptake.

- Obtain thermodynamic values such as $\mathrm{E}_{\mathrm{a}}, \Delta H^{\ominus}, \Delta S^{\ominus}$ and $\Delta G$ for the adsorption of $\mathrm{Cu}^{2+}$ which will allow understanding of what is occurring in the system and practical information on the dependence with temperature variations.

- Study the continuous adsorption of $\mathrm{Cu}^{2+}$ using a conventional axial flow column and a custom built axial flow column. Establish optimum sizing and operation for pilot scale operation. 


\section{Chapter 2 , Experimental Procedure}

The work presented in this $\mathrm{PhD}$ thesis is mainly based on the sorption of $\mathrm{Cu}^{2+}$ and the release of $\mathrm{OH}^{-}, \mathrm{Ca}^{2+}$ and $\mathrm{SiO}_{4}{ }^{4-}$ from the NCaSil in batch or continuous setups using columns packed with NCaSil. It also includes the characterisation of a real mining waste and the application of $\mathrm{NCaSil}$ as an adsorbent to recover $\mathrm{Cu}^{2+}$ from simulated waste. Below are the different procedures and techniques used in this work to prepare, execute and retrieve experimental data.

\subsection{NCaSil preparation}

\subsubsection{Reagents}

- Sodium silicate, ORICA with $28.5 \%$ wt. content as $\mathrm{SiO}_{2}, \mathrm{~N}$ grade.

- Calcium hydroxide, $\mathrm{Ca}(\mathrm{OH})_{2}$, acquired from Merck KGaA with a $96 \%$ purity.

- Distilled water, produced in the laboratory.

- Hydrochloric acid, $\mathrm{HCl}$, from Sigma Aldrich, 37\% wt., analytical grade.

- Ethanol, technical grade. 


\subsubsection{Procedure to manufacture NCaSil}

The procedure was identical to the one utilized by Cairns and McFarlane in their theses $[36,37] \mathrm{In}$ a $500 \mathrm{~mL}$ plastic beaker $15.34 \mathrm{~g}$ of sodium silicate were weighed and subsequently $250 \mathrm{~mL}$ of distilled water were added. Separately $5.32 \mathrm{~g}$ of calcium hydroxide was suspended in $250 \mathrm{~mL}$ of water using a $1 \mathrm{~L}$ plastic beaker. To this solution $4.3 \mathrm{~mL}$ of a $37 \% \mathrm{wt}$. $\mathrm{HCl}$ was added under stirring for homogenisation. Subsequently the solution containing sodium silicate was added rapidly. NCaSil is formed readily under these conditions and the suspension was left under stirring for $30 \mathrm{~min}$. The slurry was left ageing overnight allowing the formation of platelet macrostructure. After $24 \mathrm{~h}$ the material was filtered and washed with water to remove the excess of calcium, sodium and chloride ions. A final wash with ethanol using double the volume of the filter cake was performed to prevent the structure from collapsing reducing its surface area. Finally, NCaSil was dried and stored at $383 \mathrm{~K}$. 


\subsection{Kinetic studies on the adsorption $\mathrm{Cu}^{2+}$ in batch configuration}

\subsubsection{Reagents}

- Copper sulfate pentahydrate $\mathrm{CuSO}_{4} \cdot 5 \mathrm{H}_{2} \mathrm{O}$, from Carlo Erba reagents, 98\% purity.

- Distilled water, produced in our laboratory.

- NCaSil, prepared as described in Section 2.1.2.

\subsubsection{Adsorption study}

For kinetic experiments solutions of $1.6,7.9$ and $15.7 \mathrm{mmol} \mathrm{L}^{-1}$ of $\mathrm{Cu}^{2+}$ were prepared dissolving $\mathrm{CuSO}_{4} \cdot 5 \mathrm{H}_{2} \mathrm{O}$ in a $1 \mathrm{~L}$ volumetric flask. The copper solution was transferred into an Erlenmeyer flask over a heating plate equipped with magnetic stirring. After the homogenisation of the solution, $1.0000 \mathrm{~g}$ of dried NCaSil was added into the solution under a stirring speed of $500 \mathrm{rpm}$. Samples were taken from the feed and at time intervals of 1, 3, 5, 10, 15, 30, 45, 60, 90, 120, 240, $1440 \mathrm{~min}$ using a $10 \mathrm{~mL}$ polypropylene syringe. The samples were filtered through a Whatman $\mathrm{n}^{\circ} 2$ filter paper into a plastic vial and stored under refrigeration at $277 \mathrm{~K}$. Usually, samples were analysed immediately after finishing the experiment.

With the intention of having a larger amount of solids to study the kinetics of crystal growth using powder-XRD techniques, the experiments were done in a similar manner as described above using concentrations of $15.7 \mathrm{mmol} \mathrm{L}^{-1}$ of $\mathrm{Cu}^{2+}$. The entire content of the flask was filtered at a certain time. The filter cake was dried in open air for $72 \mathrm{~h}$ and stored inside glass vials.

This methodology was repeated for all studied temperatures ranging from 277 to $343 \mathrm{~K}$. For experiments below the room temperature (293 K) a mixture of water and ice was placed inside a metallic pot to cool down the flask containing the copper solution. A temperature of $277 \mathrm{~K}$ was reached with this setup. In the case of the experiments carried out above the room temperature a hot plate equipped with a thermostat was used. At the beginning of the project this equipment was not available and the temperature was stabilized on a standard hot plate adjusting the knob until the desired temperature was reached.

Stirring speeds of $50 \mathrm{rpm}$ were also studied in order to get information on the importance of this parameter on the adsorption kinetics. 


\subsection{Study of the equilibrium of $\mathrm{Cu}^{2+}$ adsorption in batch configuration}

Below is the procedure to study the equilibrium of the sorption and generate experimental data that will help to understand the thermodynamics and mechanism of the adsorption $\mathrm{Cu}^{2+}$ and the release of $\mathrm{Ca}^{2+}, \mathrm{OH}^{-}$and $\mathrm{Si}(\mathrm{OH})_{4}$.

\subsubsection{Reagents}

- Copper sulfate pentahydrate $\mathrm{CuSO}_{4} \cdot 5 \mathrm{H}_{2} \mathrm{O}$, from Carlo Erba reagents, $98 \%$ purity.

- Distilled water, produced in our laboratory.

- NCaSil, prepared as described in Section 2.1.2.

\subsubsection{Adsorption studies}

The structure of commonly used adsorbents such as ion exchange resins or activated carbon remain unvaried when they are subjected to operational conditions. In contrast, NCaSil disaggregates and dissolves depending on the chemical $(\mathrm{pH})$ and physical (stirring intensity and temperature) conditions of the media. Therefore, it was necessary to complement two different experimental approaches to obtain data with higher quality. Procedure A was the first attempt to generate data from the equilibrium using a shaking water bath. Although the shaking mechanism worked, it was not strong enough to disaggregate lumps of NCaSil.

To obtain better results, procedure $\mathrm{B}$ was done using a magnetic stirrer to disintegrate the lumps and expose fresh surface to the solution.

\section{Procedure A}

Copper sulfate solutions with concentrations of 2.0, 3.9, 4.7, 6.3, 7.1, 7.9, 11.8 and $15.7 \mathrm{mmol} \mathrm{L}^{-1}$ of $\mathrm{Cu}^{2+}$ were prepared with $\mathrm{CuSO}_{4} \cdot 5 \mathrm{H}_{2} \mathrm{O}$. The salt was dissolved in distilled water in a beaker before transferring it into a $1 \mathrm{~L}$ volumetric flask and completing the remaining volume with water.

In a $125 \mathrm{~mL}$ sealable plastic vessel $100 \mathrm{~mL}$ of stock solution were added and subsequently $0.1000 \mathrm{~g}$ of NCaSil was added into the solution. The plastic vessel was closed with its cap and placed inside a shaking water bath. Five hours elapsed before the samples were taken out and a portion immediately filtered through a whatmann $\mathrm{n}^{\circ} 2$ filter paper directly into a $25 \mathrm{~mL}$ plastic container. The filtrate was stored in the 
fridge at $277 \mathrm{~K}$. The filter cake was air dried for $72 \mathrm{~h}$ to be analysed and subsequently stored in glass vials.

\section{Procedure B}

Solutions of different concentrations were prepared in $2 \mathrm{~L}$ portions in order to have enough solution (1.5 L total) for all 3 temperatures. These solutions had concentrations of $2.0,3.9,4.7,6.3,7.1,7.9,11.8$ and $15.7 \mathrm{mmol} \mathrm{L}^{-1}$ of $\mathrm{Cu}^{2+}$, and were prepared using $\mathrm{CuSO}_{4} \cdot 5 \mathrm{H}_{2} \mathrm{O}$. The salt was dissolved in a beaker and subsequently transferred into a volumetric flask where the remaining volume was completed with distilled water.

In a measuring cylinder $500 \mathrm{~mL}$ of the solution were added and subsequently transferred into an Erlenmeyer flask. The solution was stirred over a hot plate equipped with magnetic stirring and a thermostat. $0.5000 \mathrm{~g}$ of dried NCaSil was weighed. The solid was poured into the solution under a stirring speed of $500 \mathrm{rpm}$. After $24 \mathrm{~h}$ the entire contents of the flask were filtered through a Whatmann $\mathrm{n}^{\circ} 2$ filter paper with a Büchner funnel and flask, using vacuum filtration.

The filtrate was stored in a sealed plastic vial inside the fridge at $277 \mathrm{~K}$ and the filter cake was dried open air for $72 \mathrm{~h}$ before placing it inside a glass vial.

This procedure was repeated when the reaction was studied at 303 and $313 \mathrm{~K}$. 


\subsection{Mining Waste Collection and Treatment}

\subsubsection{Mining Waste Collection}

Two $1 \mathrm{~L}$ samples of Caletones smelter weak acid were collected by CODELCO staff at El Teniente mine $\left(34^{\circ} 07^{\prime} \mathrm{S}\right.$ and $\left.70^{\circ} 27^{\prime} \mathrm{W}\right)$ at $11 \mathrm{AM}(\mathrm{GMT}-4)$ on the $9^{\text {th }}$ of August 2010. Both samples were stored in a cooler carry-bag and taken to Hidrolab ltda. in Santiago, Chile, a private laboratory holding ISO 17025 certification. The sample containing suspended solids was shipped to Victoria University of Wellington, New Zealand and Georg-Simon-Ohm Hochschule in Germany for further analyses.

A daily analysis of the weak acid effluent was undertaken on site by the acid plant operator. In this work, data collected from May 2010 until October 2010 is presented. Control charts were plotted (presented in the appendix) for the data in order to monitor and understand the long-term operation of the sulfuric acid plant, the quality of the weak acid being discharged and to place the samples collected into context. The charts present the average value for the variables analysed over time. The lower and upper warning limits (LWL and UWL), which are chosen to be twice the standard deviation $( \pm 2 \sigma)$ from the average, are also presented. The control charts also include the lower and upper action limits (LAL and UAL) defined to be three times the standard deviation $( \pm 3 \sigma)$. Approximately $99.7 \%$ of the results fall inside these limits. If any data lies outside the LAL or UAL immediate action should be taken to bring the operational output back close to the average.

\subsubsection{Preparation of a simulated mining waste}

After characterising the liquid portion of the waste (refer to Section 2.7.3.1) the acquired data were used to emulate the waste with the main components that were present in concentrations $>1 \mathrm{mg} \mathrm{L}^{-1}$. Aluminium was excluded as it formed a precipitate when it was added to the multicomponent solution. Trying to match the counter ion concentration with those reported on the analysis was a challenge, therefore if an excess was to happen usually nitrate salts were used in order to prevent the formation of a precipitate. 
A total volume of $1 \mathrm{~L}$ of mining waste was prepared using the salts and amounts detailed in Table 2.1.

Table 2.1: Reagents for the preparation of the weak acid

\begin{tabular}{|c|c|c|c|}
\hline Analyte & Source & $\begin{array}{c}\text { Mass added } \\
{[\mathrm{mg}]}\end{array}$ & $\begin{array}{c}\text { Concentration } \\
{\left[\mathrm{mg} \mathrm{L}^{-1}\right]}\end{array}$ \\
\hline $\mathrm{Cu}$ & $\mathrm{CuSO}_{4} \cdot 5 \mathrm{H}_{2} \mathrm{O}$ & 2044 & 510 \\
\hline $\mathrm{Ca}$ & $\mathrm{Ca}\left(\mathrm{NO}_{3}\right)_{2}$ & 6327 & 1530 \\
\hline $\mathrm{Fe}$ & $\mathrm{FeSO}_{4} \cdot 7 \mathrm{H}_{2} \mathrm{O}$ & 597.4 & 120 \\
\hline $\mathrm{Pb}$ & $\mathrm{Pb}\left(\mathrm{NO}_{3}\right)_{2}$ & 49.6 & 31 \\
\hline Mo & $\left(\mathrm{NH}_{4}\right)_{6} \mathrm{Mo}_{7} \mathrm{O}_{24} \cdot 4 \mathrm{H}_{2} \mathrm{O}$ & 95.7 & 52 \\
\hline \multirow[t]{2}{*}{$\mathrm{As}\left[\mathrm{AsO}_{4}{ }^{3-}\right]$} & $\mathrm{Na}_{3} \mathrm{AsO}_{4}$ & 22586 & 8140 \\
\hline & $\mathrm{H}_{2} \mathrm{SO}_{4}$ & 64369 & \multirow{3}{*}{65453} \\
\hline \multirow[t]{2}{*}{$\mathrm{SO}_{4}^{2-}$} & $\mathrm{CuSO}_{4} \cdot 5 \mathrm{H}_{2} \mathrm{O}$ & 0.1 & \\
\hline & $\mathrm{FeSO}_{4} \cdot 7 \mathrm{H}_{2} \mathrm{O}$ & 0.02 & \\
\hline \multirow[t]{2}{*}{$\mathrm{NO}_{3}^{-}$} & $\mathrm{Ca}\left(\mathrm{NO}_{3}\right)_{2}$ & 4782 & \multirow{2}{*}{4800} \\
\hline & $\mathrm{Pb}\left(\mathrm{NO}_{3}\right)_{2}$ & 18.6 & \\
\hline $\mathrm{H}_{2} \mathrm{O}_{2}$ & $\mathrm{H}_{2} \mathrm{O}_{2} 30 \% \mathrm{wt}$ & 1 & 1 \\
\hline
\end{tabular}

The reagents were dissolved in a beaker before being transferred into a $1 \mathrm{~L}$ volumetric flask. Taking into consideration the $\mathrm{E}$ vs $\mathrm{pH}$ diagrams (Pourbaix diagrams) of all elements a certain amount of $\mathrm{H}_{2} \mathrm{O}_{2}$ was added into the solution to raise the potential and stabilise the elements in their soluble ionic forms preventing the formation of precipitates. The addition of calcium nitrate as a source of calcium also provides nitrate to the solution helping to keep certain cations soluble that instead would form sulfate precipitates. This action, although unwanted, was necessary due to the complexity of a multi elemental sample. After adding all dissolved salts into the flask the remaining volume was completed with distilled water. The resulting $\mathrm{pH}$ value of the solution was -0.1 .

\subsubsection{Emulated Mining waste Treatment.}

The treatment of the emulated mining waste consists in 4 steps: first, raising the $\mathrm{pH}$ to prevent NCaSil from hydrolysing; second, solid liquid separation by vacuum filtration; third, addition of NCaSil to remove $\mathrm{Cu}^{2+}$ from solution; and fourth, a final solid-liquid separation by vacuum filtration to retrieve the $\mathrm{Cu}^{2+}$ enriched solid and discharge of depleted liquid. 
An aliquot of $500 \mathrm{~mL}$ of the emulated mining waste solution was transferred into a 2 L Erlenmeyer flask using a measuring cylinder. Subsequently the flask was placed over a magnetic stirrer.

Taking into consideration that NCaSil is completely hydrolysed at $\mathrm{pH}$ values $<2$, the first step involved raising the $\mathrm{pH}$ from its initial value of -0.1 to 3 by adding $600 \mathrm{~mL}$ of a $30 \%$ wt. milk of lime $\left(\mathrm{Ca}(\mathrm{OH})_{2}\right.$ suspension). The reaction was allowed to stand for 15 minutes and a white precipitate developed over this time. The suspension was filtered using a typical vacuum filtration setup. The filter cake was dried and stored at $383 \mathrm{~K}$ inside a porcelain dish and the filtrate transferred into a new $2 \mathrm{~L}$ flask.

The next step of the treatment consisted in adding $1.0019 \mathrm{~g}$ of NCaSil into the flask containing $\mathrm{pH}$ adjusted emulated mining waste filtrate. The solution was stirred for $24 \mathrm{~h}$ and then filtered. The filtrate was stored in a plastic container at $277 \mathrm{~K}$ and the filter cake was dried in open air for $72 \mathrm{~h}$ inside the hood and stored inside a glass vial.

The filtrate and the filter cake were analysed using the same techniques as those mentioned in Section 2.7. 


\subsection{Continuous adsorption studies}

Two different columns were tested. The first one was a conventional axial flow column which was operated in a downward flow. The second was a custom build radial flow column (RFC), which was operated in outward flow and by immersion in a tank containing copper ions in solution.

\subsubsection{Reagents}

- Copper sulfate pentahydrate, from Carlo Erba reagents, 98\% purity.

- Distilled water, produced in our laboratory.

- NCaSil, prepared as described in Section 2.1.2.

\subsubsection{Conventional axial flow column}

The column was made out of glass having a total length of $25 \mathrm{~cm}$, of which $14 \mathrm{~cm}$ can be packed. The internal column diameter was $2 \mathrm{~cm}$. Both ends of the column were sealed by a septum. The bottom septum, located at the outlet, had a filter paper placed between two polyurethane meshes as a packing support. Into each septum a hole was drilled on the centre and a $4 \mathrm{~cm}$ piece of nylon tubing with an internal diameter of $2 \mathrm{~mm}$ was inserted. The tubing coming from the pump was attached to the nylon tubing of the column by plastic moorings. The system proved to be leak proof under the experimental conditions studied. An example of this packed column is displayed in Figure 2.1.

\subsubsection{Operational procedure for the conventional axial flow column}

The column was tested using three different concentrations and flows, and two different heights. The last one given by the amount of NCaSil added to the column. Different packing densities could not be studied due to the high back pressures generated when the material was compressed.

In order to pack the column either 0.5 or $1.0 \mathrm{~g}$ of $\mathrm{NCaSil}$ were added using a funnel to the column on top of the packing support. Subsequently a round filter paper cut to fit inside the column was placed on top of the NCaSil. Afterwards, $1 \mathrm{~cm}$ of glass beads was placed on top to prevent the NCaSil moving and equalize flow distribution inside the column. Figure 2.1 shows the typical packed column. 


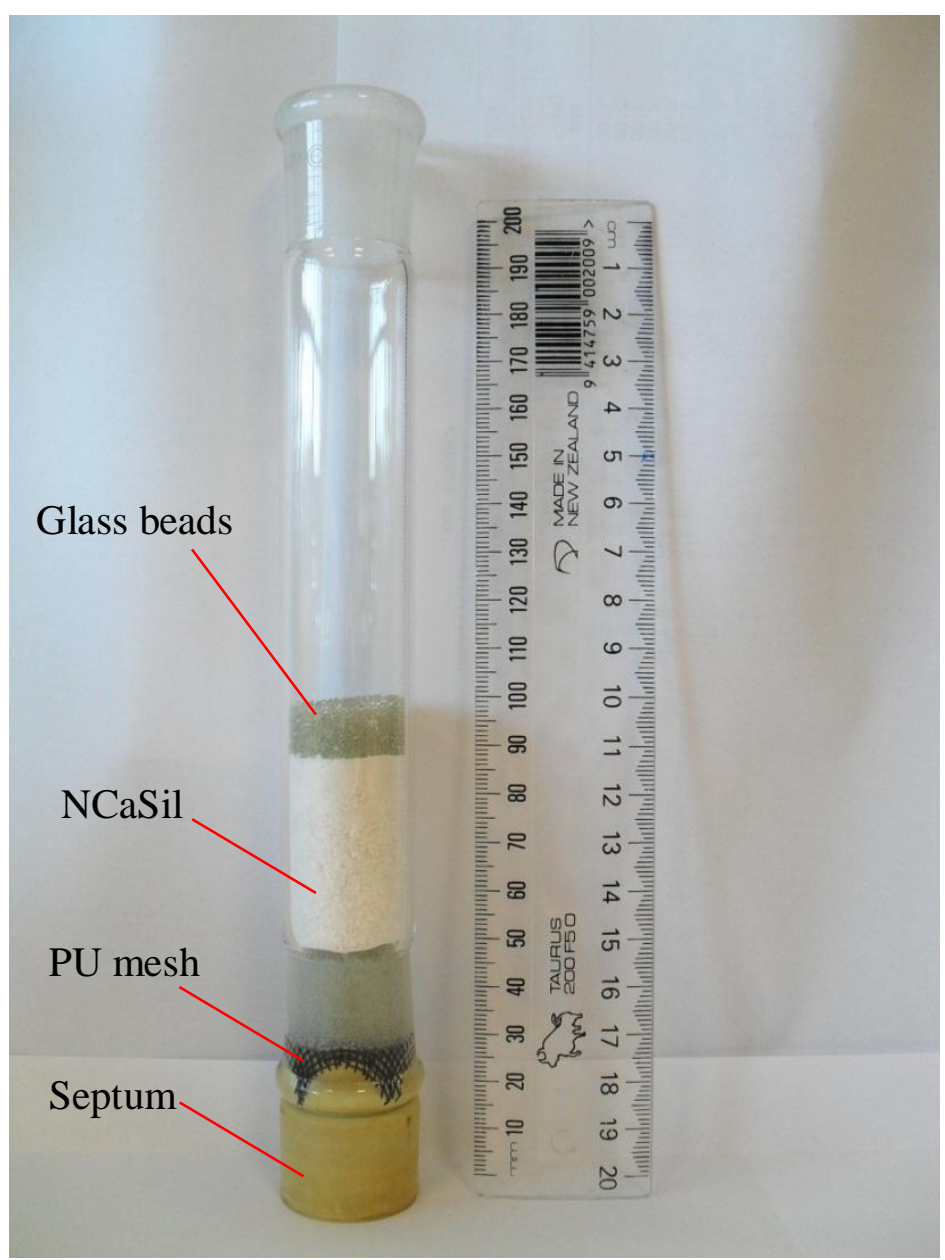

Figure 2.1: Packed column with NCaSil

The solution containing $\mathrm{Cu}^{2+}$ was placed inside a $2 \mathrm{~L}$ plastic beaker. The feed tubing of the pump was submerged inside the solution and liquid was pumped to the inlet of the column. The inlet was opened rapidly and a portion of the solution was used to fill the empty space left from glass beads to the inlet. Subsequently, a quick manoeuvre was done to close the inlet again. The inlet and the outlet septum were fastened with nylon cable ties to prevent them from moving when pressure was accumulated.

Flows of $5.00 \mathrm{ml} \mathrm{min}^{-1}$ were pumped using an HPLC Merck-Hitachi L-6000A pump. Column heights of 2.5 and $5 \mathrm{~cm}(0.5$ and $1.0 \mathrm{~g}$ of NCaSil $)$ were tested. Concentrations of 1.6, 7.9 and $15.7 \mathrm{mmol} \mathrm{L}^{-1}$ of $\mathrm{Cu}^{2+}$ were employed in the study.

The typical setup for this study is shown Figure 2.2. 


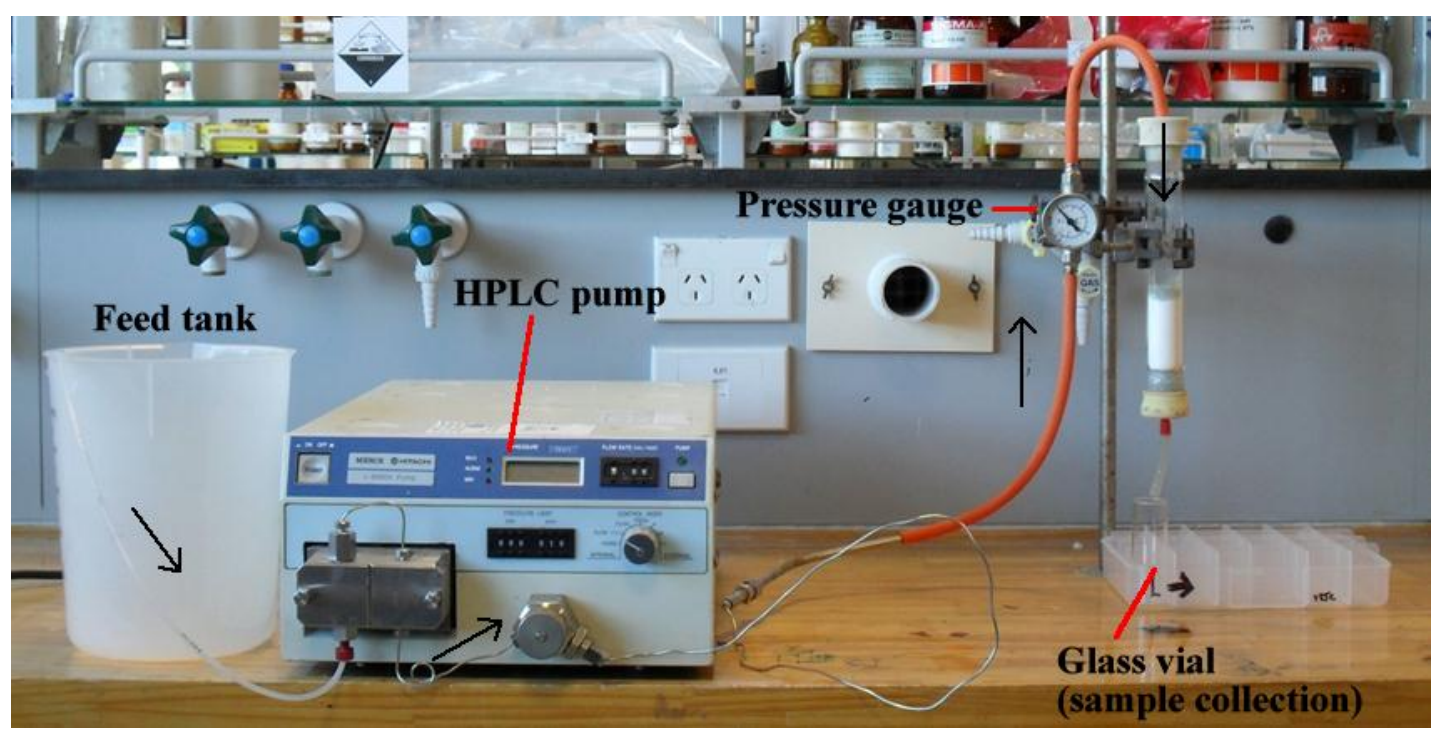

Figure 2.2: Common arrangement for the studies done with a conventional axial flow column packed with NCaSil. The black arrow on the picture represents the direction of flow.

\subsubsection{Radial flow column (RFC)}

Nano structured calcium silicate has a small particle size. Due to this using such material inside a standard column will result in a large pressure drop through the column. Thus, for a larger scale application another design has to be used. Based upon existing microfiltration systems [48], activated carbon block filters [49], and industrial chromatography [50-52] a custom made radial flow column (RFC) was built. The device components are shown in Figure 2.3.

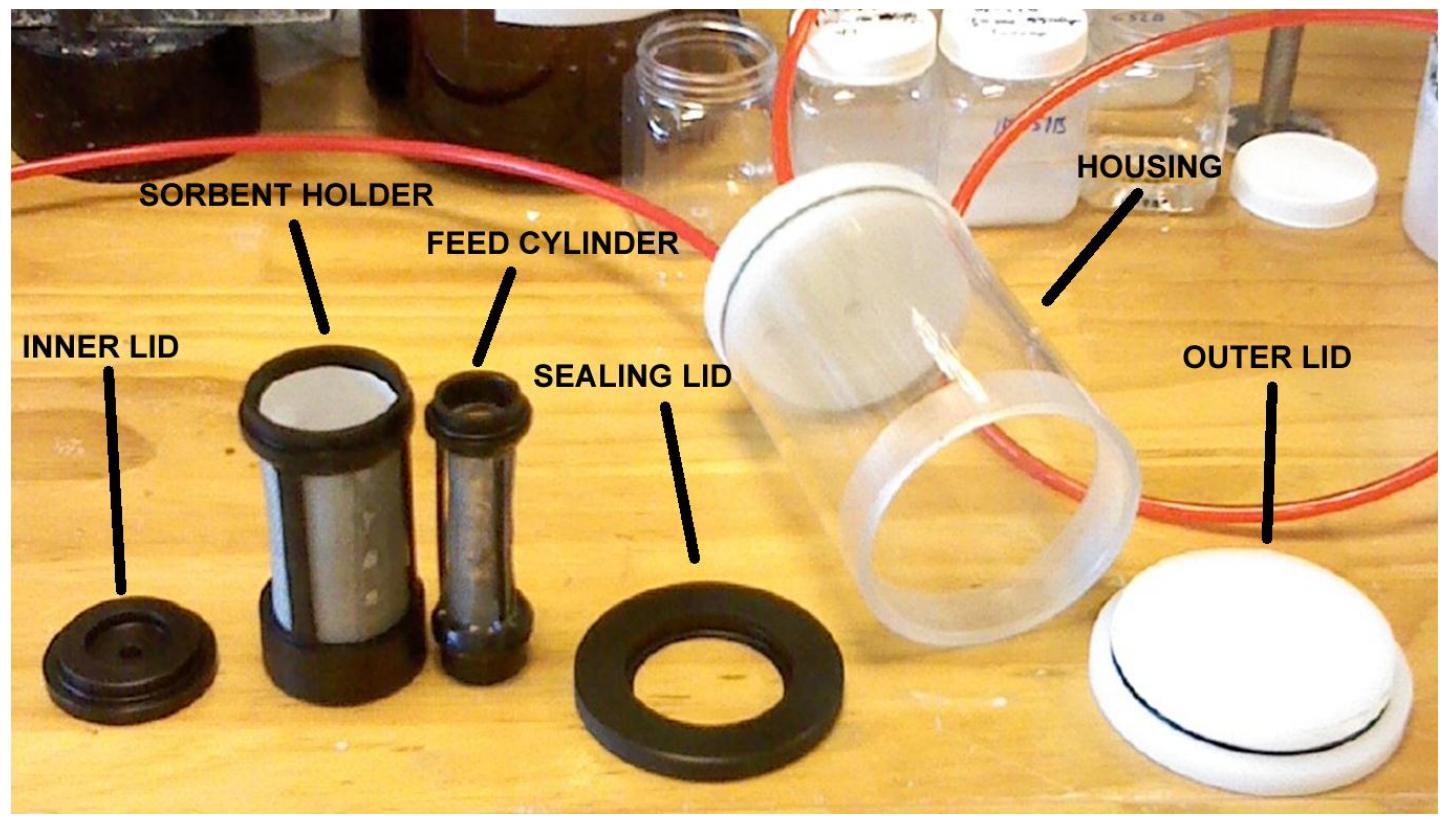

Figure 2.3, Components of the RFC. The column may be used only with the sorbent holder, feed cylinder and inner lid, without the housing and outer components.

This custom built RFC is made out of three concentric cylinders. The adsorbent is placed between the first (feed cylinder) and the second (sorbent holder) cylinder 
while the third cylinder (transparent housing in Figure 2.3) acts as an outer casket creating a closed system. Water enters through the centre of the first cylinder and flows radially through the mantle, crossing the packed material towards the outer casket. The measures of the different elements are as follows: inner feed cylinder, $D_{\text {int }} 2 \mathrm{~cm}$; sorbent holder diameter $D_{S B} 4 \mathrm{~cm}$; internal length, $C_{L} 8 \mathrm{~cm}$. Therefore the ratio of $D_{\text {int }}: D_{S B}: C_{L}=a: 2 a: 4 a$, where $a$ is the length of the inner feed cylinder as shown in Figure 2.4.

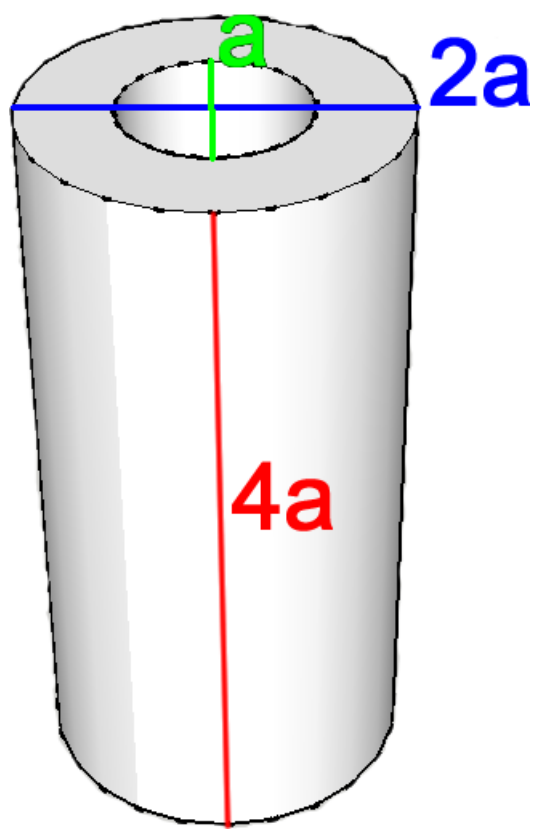

Figure 2.4: Ratio of the inner feed cylinder diameter to the sorbent holder diameter to the cylinder to the length. The ratio is a:2a:4a.

This design offers increased cross sectional area decreasing the hydraulic loading over the column. Furthermore, operating a column radially grants the possibility of having the same amount of material as a column without having the large pressure drops associated with columns. Although the shorter bed depth implies shorter residence times, this can be overcome if the material has a high rate of uptake of the pollutant.

\subsubsection{Procedure to pack the RFC with NCaSil}

A $14 \times 8 \mathrm{~cm}$ rectangle of filter paper was cut as packing support which lay over the stainless steel mesh of the sorbent holder. The paper was sprayed with water in order to fix it to the surface of the sorbent holder. The feed cylinder was then inserted into the sorbent holder and subsequently $3 \mathrm{~g}$ of NCaSil were added with a spatula in small portions. Every 4 portions added the sorbent holder was bumped slightly against the surface of the bench in order to guarantee a tighter packing and minimize free channel formation during the operation. 


\subsubsection{RFC operating as column}

The solution containing $\mathrm{Cu}^{2+}$ was placed inside a $2 \mathrm{~L}$ plastic beaker. The feed tubing of the pump was submerged inside the solution and liquid was pumped to the inlet of the column. An example of such procedure is shown in Figure 2.5.

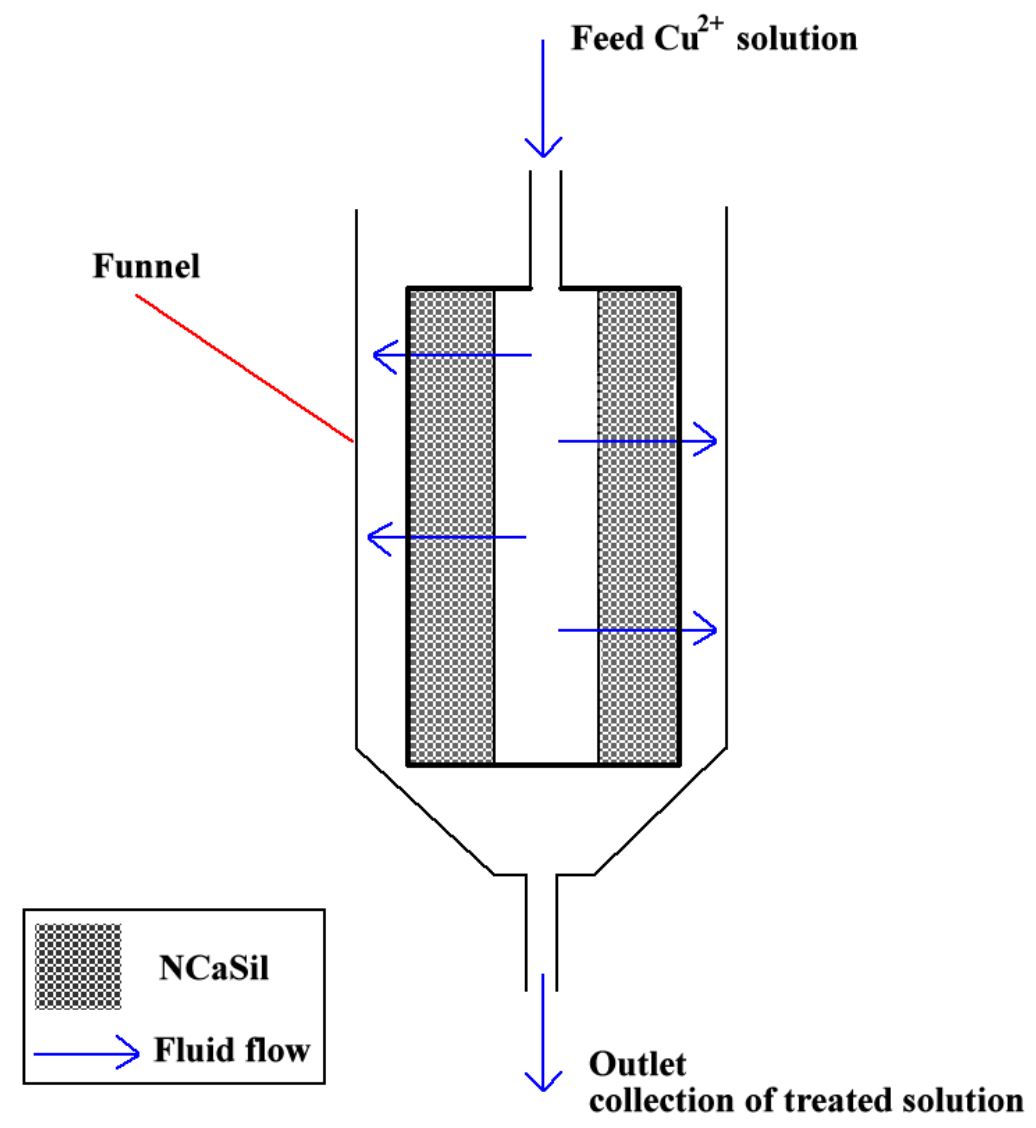

Figure 2.5, Cross-sectional scheme of the continuous sorption device during operation. The setup was the same as the shown in Figure 2.2 for the conventional axial flow column.

A flow of $10 \mathrm{ml} \mathrm{min}^{-1}$ was used utilizing a HPLC Merck-Hitachi L-6000A pump. Concentrations of 1.6, 7.9 and $15.7 \mathrm{mmol} \mathrm{L}^{-1}$ of $\mathrm{Cu}^{2+}$ were employed in the study. The bed width was $2 \mathrm{~cm}$ which is set by the gap between the sorbent holder and the inner feed cylinder. This gives the RFC a logarithmic mean area of $0.0073 \mathrm{~m}^{2}$ (refer to eq. 5.7 in section 5.1.1.4). Therefore, in this study a hydraulic loading of $1.37 \times 10^{-3} \mathrm{~m}^{3} \mathrm{~m}^{-2} \mathrm{~min}^{-1}$ was used (refer to section 5.1.1 for further explanation). In this study the housing was replaced by an open air funnel which helped to collect the treated effluent. Using the housing would decrease the resolution of the collected data due to the accumulation of the solution between the sorbent holder and the housing wall. Hence, using it would make very difficult to see concentration differences in the collected samples, reflecting the average concentration during a long time frame. 


\subsubsection{RFC operation by Immersion.}

The RFC packed with $3.0 \mathrm{~g}$ of NCaSil was immersed in a stirred tank containing $3 \mathrm{~L}$ of a $\mathrm{Cu}^{2+}$ solution. Once inside the solution was recirculated through the device using a flow of $200 \mathrm{ml} \mathrm{min}{ }^{-1}$ utilizing a masterflex peristaltic pump, model \# 7521-25, equipped with a head model number 7016-20. Concentrations of $1.6,7.9 \mathrm{mmol} \mathrm{L}^{-1}$ of $\mathrm{Cu}^{2+}$ were employed in the study. The bed width was $2 \mathrm{~cm}$ which is set by the gap between the sorbent holder and the inner feed cylinder. The RFC has logarithmic mean area of $0.0073 \mathrm{~m}^{2}$ (refer to eq. 5.7 in section 5.1.1.4). Therefore, in this study a hydraulic loading of $1.6 \mathrm{~m}^{3} \mathrm{~m}^{-2} \mathrm{~h}^{-1}$ was used. An example of this setup is presented in Figure 2.6.

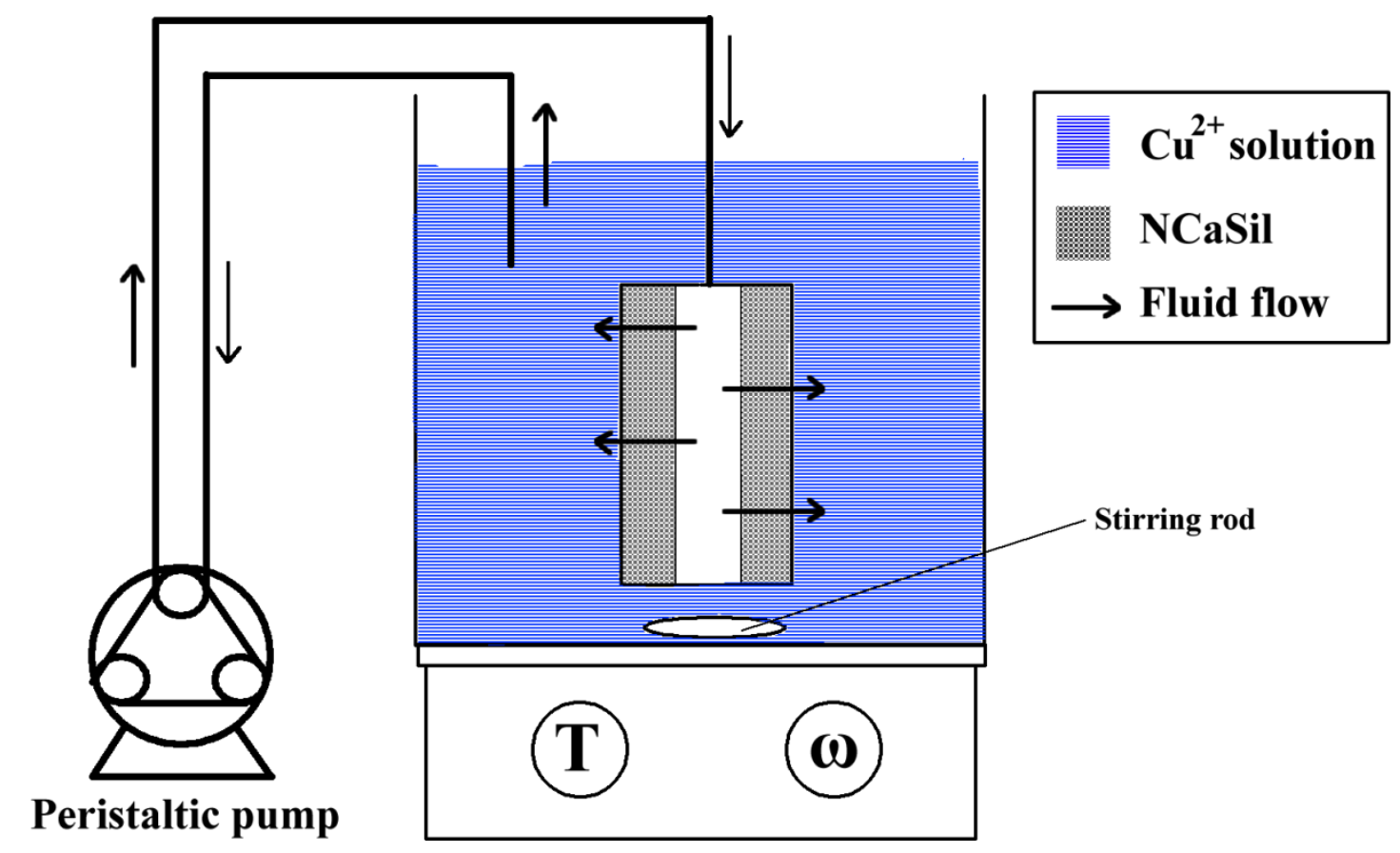

Figure 2.6: RFC operation by immersion. The figure shows the RFC submerged in a stirred tank containing a $\mathrm{Cu}^{2+}$ solution. The solution is being recirculated by a peristaltic pump. 


\subsection{Sample Characterisation}

\subsubsection{Liquid sample characterisation, $\mathrm{Cu}^{2+}$ sorption kinetic experiments}

\subsubsection{Measurement of $\mathrm{Cu}^{2+}$}

The concentration of copper ions in the filtrate was measured at $324.7 \mathrm{~nm}$ using a Flame Atomic Absorption Spectrophotometre (FAAS) GBC 906 using an air-acetylene flame. All measurements were carried out by diluting an aliquot inside a glass vial with water delivered by a micropipette. This allows the analysis of a large number of samples without the need to have a large number of volumetric flasks. To improve the statistics all samples were measured in triplicate. Normally, a dilution factor ranging from 21 to 51 was needed for those samples taken during the first 15 to 30 minutes in the experiments using feed solutions containing an initial concentration of $\mathrm{Cu}^{2+}$ of 100 and $500 \mathrm{mg} \mathrm{L}^{-1}$. In the case of the studies with a 1000 $\mathrm{mg} \mathrm{L}^{-1}$ of $\mathrm{Cu}^{2+}$ solution, a dilution factor of 201 was necessary. Therefore, an aliquot of $25 \mu \mathrm{L}$ was placed inside a glass vial and $5 \mathrm{~mL}$ of distilled water were added on top.

A calibration curve was constructed ranging from 1 to $5 \mathrm{mg} \mathrm{L}^{-1}$ of dissolved copper, as recommended by the instrument manual. The standards were made using a stock solution containing $\mathrm{Cu}^{2+}$ stabilized in $3 \%$ wt of $\mathrm{HNO}_{3}$ from Fluka, TraceCERT® quality which were permanently stored at $277 \mathrm{~K}$ inside a fridge. The blank was distilled water, the same quality as the one used for the study.

The concentration of the feed was measured as well all other samples. Equation 2.1 was used to calculate the concentration of copper on the surface.

$q_{t}=\frac{\left(\left[\mathrm{Cu}^{2+}\right]_{\text {feed }}-\left[\mathrm{Cu}^{2+}\right]_{t}\right)}{m_{\text {NCaSil }}} \cdot V$

Where, $q_{t}$ is the amount adsorbed at time $\mathrm{t}$ in $\mathrm{mmol} \mathrm{g}^{-1},[\mathrm{Cu}]_{\text {feed }}$ is the starting concentration of $\mathrm{Cu}^{2+}$ in solution in $\mathrm{mmol} \mathrm{L}^{-1},[\mathrm{Cu}]_{\mathrm{t}}$ is the concentration of sample in mmol L $\mathrm{L}^{-1}$ taken at time $t, m_{N C a S i l}$ is the mass of NCaSil added for the experiment in grams and $V$ is the volume of copper sulfate solution utilized in the experiment in L.

\subsubsection{Measurement of $\mathrm{Ca}^{2+}$}

To avoid chemical interferences a solution of $2000 \mathrm{mg} \mathrm{L}^{-1}$ of $\mathrm{Sr}^{2+}$ was prepared by dissolving $6.07 \mathrm{~g}$ of strontium chloride, 99\% purity, from Sigma Aldrich in $200 \mathrm{~mL}$ 
of distilled water in a $250 \mathrm{~mL}$ beaker. The solution was then transferred into a $1 \mathrm{~L}$ volumetric flask and the remaining volume completed with distilled water.

A $1000 \mathrm{mg} \mathrm{L}^{-1} \mathrm{CaCO}_{3}$ solution was prepared using $3.5 \mathrm{~g}$ of analytical grade calcium carbonate, from BDH Chemicals Ltd with $99 \%$ purity. The salt was dried for $24 \mathrm{~h}$ at $383 \mathrm{~K}$ and placed in a desiccator to cool. Once cooled, $2.5225 \mathrm{~g}$ were weighed and transferred into a $200 \mathrm{~mL}$ beaker. Subsequently a $3 \%$ wt. $\mathrm{HNO}_{3}$ solution was poured gently on top and stirred. Once calcium carbonate was dissolved the solution was transferred into a $1 \mathrm{~L}$ volumetric flask and the volume made up with the $3 \%$ wt. of $\mathrm{HNO}_{3}$. Standards ranging from 1 to $4 \mathrm{mg} \mathrm{L}^{-1}$ of $\mathrm{Ca}^{2+}$ were prepared from this stock solution by dilution with a $2000 \mathrm{mg} \mathrm{L}^{-1}$ of $\mathrm{Sr}^{2+}$ solution.

Usually a dilution factor of 51 was needed to be within the calibration curve. Therefore, an aliquot of $100 \mu \mathrm{L}$ was transferred into a glass vial using a micropipette and $5 \mathrm{~mL}$ of distilled water were subsequently added with a micropipette.

The concentration of calcium ions in the filtrate of the collected samples was measured at $422.7 \mathrm{~nm}$ using a FAAS GBC 906 using air acetylene in triplicate. The concentration of calcium was calculated using Equation 2.2.

$q_{r, t, C a}=\left([C a]_{t}-[C a]_{B l a n k}\right)$

Where $q_{, r, t, C a}$ is the concentration in mmol of $\mathrm{Ca}^{2+}$ per $\mathrm{L}$ released at time $t,[\mathrm{Ca}]_{t}$ is the concentration of calcium that has been released at time $t$ in $\mathrm{mmol} \mathrm{L}^{-1},[\mathrm{Ca}]_{\text {Blank }}$ is the concentration of calcium in the blank in $\mathrm{mmol} \mathrm{L}^{-1}$,

\subsubsection{Measurement of Monomeric Silica}

The concentration of monomeric silica in solution was determined using silicomolybdate method recommend by IIer [53]. This method was successfully used for the analysis of NCaSil by McFarlane [36], although the method had to be modified in order to be able to analyse a large number of samples.

This method involves the formation coloured silicomolybdic acid due to the reaction of ammonium molybdate and monomeric silica in an acid media. The colouration allows quantifying the analyte spectrophotometrically. The reaction described above is represented in Equation 2.3.

$7 \mathrm{Si}(\mathrm{OH})_{4}+12 \mathrm{H}_{6} \mathrm{Mo}_{7} \mathrm{O}_{24} 4 \mathrm{H}_{2} \mathrm{O}+126 \mathrm{H}_{2} \mathrm{O} \rightleftharpoons 7 \mathrm{H}_{8} \mathrm{Si}\left(\mathrm{Mo}_{2} \mathrm{O}_{7}\right)_{6} 28 \mathrm{H}_{2} \mathrm{O}$ 
In strong acid media molybdate becomes protonated forming molybdic acid in solution. This reagent is only stable for one week so it has to be prepared when required from other two stock solutions as detailed below.

Reagent A: Add $4.0 \mathrm{~mL} 95.5 \%$ (w/w) sulfuric acid to $800 \mathrm{~mL}$ double distilled water and dilute $1 \mathrm{~L}$.

Reagent B: Dissolve $100 \mathrm{~g}$ of ammonium molybdate (VI) tetrahydrate in $900 \mathrm{~mL}$ double distilled water, add $47 \mathrm{~mL} \mathrm{28 \%} \mathrm{(w/w)} \mathrm{ammonium} \mathrm{hydroxide,} \mathrm{and} \mathrm{dilute} \mathrm{to} 1$ L.

Reagents C: Place $500 \mathrm{~mL}$ of double distilled water in a plastic container and add $200 \mathrm{~mL}$ of reagent $\mathrm{A}$ and $100 \mathrm{~mL}$ of reagent $\mathrm{B}$.

With the intention of analysing $>10$ samples at the same time and minimizing the use of reagent $\mathrm{C}$ the analytes were prepared directly into the cuvette using micropipettes.

Sufficient UV-Vis perspex cells to analyse the samples were placed inside a cuvette holder and $0.4 \mathrm{~mL}$ of water were added to each using a $100-1000 \mu \mathrm{L}$ micropipette. On top $0.4 \mathrm{~mL}$ of sample were added into each cuvette. Finally $3.2 \mathrm{~mL}$ of reagent $\mathrm{C}$ were added using a 1000-5000 $\mu \mathrm{L}$ micropipette. Standards of Si were prepared utilizing a stock standard solution of $1000 \mathrm{mg} \mathrm{L}^{-1} \mathrm{Si}$ from Fluka, TraceCERT®. A $100 \mathrm{mg} \mathrm{L}^{-1}$ of $\mathrm{Si}$ solution was prepared from the stock solution. In a similar manner the standards were prepared as detailed in as shown in Table 2.2.

Table 2.2: preparation of standards for monomeric silica measurements.

\begin{tabular}{|c|c|c|c|}
\hline $\begin{array}{l}\text { conc. of } \\
\text { standard } \\
{\left[\mathrm{mg} \mathrm{L}^{-1}\right]}\end{array}$ & $\begin{array}{c}\text { aliquot of } \\
100 \mathrm{mg} \mathrm{L}^{-1} \mathrm{Si} \text { standard. } \\
{[\mathrm{ml}]}\end{array}$ & $\begin{array}{c}\text { volume of } \\
\text { distilled water } \\
{[\mathrm{ml}]}\end{array}$ & $\begin{array}{l}\text { volume of } \\
\text { reagent c } \\
\text { [ml] }\end{array}$ \\
\hline 2.5 & 0.1 & 0.7 & 3.2 \\
\hline 5.0 & 0.2 & 0.6 & 3.2 \\
\hline 7.5 & 0.3 & 0.5 & 3.2 \\
\hline 10 & 0.4 & 0.4 & 3.2 \\
\hline
\end{tabular}

The blank was prepared adding $3.2 \mathrm{~mL}$ of reagent $\mathrm{C}$ onto $0.8 \mathrm{~mL}$ of distilled water, having the same quality as the one used in the adsorption experiments.

It is important that reagent $\mathrm{C}$ is added to all samples after the addition of the blank and standards to assure that the formation of silicomolybdic acid starts almost at the same time in each cuvette. Since the beta form of silicomolybdic acid that forms at 
the beginning is more coloured than the alpha form [36], the absorbance was measured 10 minutes after reagent $\mathrm{C}$ was added into solution. This time is enough to allow the colour to fully develop and short enough so it doesn't fade. The absorbance of the solution was measured at $410 \mathrm{~nm}$ using either Shimadzu UV-160 or Varian Cary 100SCAN UV-Visible spectrophotometer. This wavelength offered a good range of linearity that obeyed Beer's Law.

The concentrations of monomeric silica were obtained as $\mathrm{mg} \mathrm{L}^{-1}$ of $\mathrm{Si}$ in solution; therefore a conversion was done using Equation 2.4 to express it as $\mathrm{mg}$ of $\mathrm{SiO}_{2}$ per L.

$q_{r, t, S i O_{2}}=\left(\frac{[S i]}{A_{r}(S i)}\right) x 10$

Where $q_{r, t, S i O 2}$ is the amount of monomeric silica released per gram of adsorbent at time $t$ expressed as $\mathrm{SiO}_{2}$ in $\mathrm{mmol} \mathrm{g}^{-1}$, [Si] is the concentration reported by the spectrophotometer for $\mathrm{Si}$ in $\mathrm{mg} \mathrm{L}^{-1}, A_{r}(\mathrm{Si})$ is the relative atomic mass of silicon. The equation is multiplied by 10 which is the dilution factor arising from the dilution of $0.4 \mathrm{ml}$ of sample in a total volume of $4 \mathrm{ml}$.

\subsubsection{Measurement of the $\mathrm{pH}$}

A $\mathrm{pH}$ electrode and a temperature probe were connected to a cyberscan $\mathrm{pH} 11 \mathrm{pH}$ metre from Eurotech Instruments. The instrument was calibrated at room temperature using standard solutions with $\mathrm{pH}$ values of 4, 7 and 10, acquired from Sigma Aldrich. The $\mathrm{pH}$ electrode was placed inside the flask with the copper sulfate solution and the initial $\mathrm{pH}$ of the reaction was recorded. Subsequently as described in Section 2.3.2 a known amount of $\mathrm{NCaSil}$ was added into the reaction vessel and the $\mathrm{pH}$ value was recorded manually every 15 seconds for the first 2 minutes; every 30 seconds during the $2^{\text {nd }}$ to $5^{\text {th }}$ minute; every one minute between the $5^{\text {th }}$ and the $15^{\text {th }}$ minute and every 5 minutes between the $15^{\text {th }}$ minute and the equilibrium time. Practically speaking the equilibrium is considered to be where the $\mathrm{pH}$ value remained unvaried for twice the time to uptake $\mathrm{Cu}^{2+}$, a condition which depended on the several variables such as $\mathrm{Cu}^{2+}$ concentration in solution and temperature.

\subsubsection{Liquid sample characterisation, equilibrium of the sorption of $\mathrm{Cu}^{2+}$}

The characterisation of the filtrate was done in a similar manner as the method described in Section 2.3.3. Although in this case the samples were taken out once the adsorption process had reached the equilibrium. 


\subsubsection{Measurement of $\mathrm{Cu}^{2+}$ at the equilibrium}

The measurement was carried out as described in Section 2.7.1.1. The amount absorbed at the equilibrium was calculated using Equation 2.5.

$$
q_{e}=\frac{\left(\left[\mathrm{Cu}^{2+}\right]_{\text {initial }}-\left[C u^{2+}\right]_{e}\right)}{m_{N C a S i l}} \cdot V
$$

Where, $q_{e}$ is the amount adsorbed at the equilibrium in $\mathrm{mmol} \mathrm{g}^{-1},[\mathrm{Cu}]_{\text {initial }}$ is the initial concentration of $\mathrm{Cu}^{2+}$ in solution in $\mathrm{mmol} \mathrm{L}^{-1},[\mathrm{Cu}]_{\mathrm{eq}}$ is the concentration of copper at the equilibrium in $\mathrm{mmol} \mathrm{L}^{-1}$ and $V$ is the volume of copper sulfate solution utilized in the experiment in L.

\subsubsection{Measurement of $\mathrm{Ca}^{2+}$ at the equilibrium}

The measurement was carried out as described in Section 2.7.1.2. The amount absorbed at the equilibrium was calculated using Equation 2.6.

$$
q_{r, e, C a}=\left([\mathrm{Ca}]_{e q}-[\mathrm{Ca}]_{\text {Blank }}\right)
$$

Where $q_{, r, e, C a}$ is the concentration of $\mathrm{Ca}^{2+}$ released at the equilibrium in $\mathrm{mmol} \mathrm{L}^{-1}$, $[\mathrm{Ca}]_{\mathrm{eq}}$ is the concentration of calcium that has been released at the equilibrium in $\mathrm{mmol} \mathrm{L}{ }^{-1},[\mathrm{Ca}]_{\text {Blank }}$ is the concentration of calcium in the blank in $\mathrm{mmol} \mathrm{L} \mathrm{L}^{-1}$.

\subsubsection{Measurement of monomeric silica at the equilibrium}

The measurement was carried out as described in Section 2.7.1.3. The concentrations of monomeric silica were obtained as $\mathrm{mg} \mathrm{L}^{-1}$ of $\mathrm{Si}$ in solution, therefore a conversion was done using Equation 2.7 to express it as $\mathrm{mmol}$ of $\mathrm{SiO}_{2}$ per $\mathrm{L}$.

$q_{r, e, S i O_{2}}=\left(\frac{[S i]}{A_{r}(S i)}\right) \cdot 10$

Where $\mathrm{q}_{r, e, S i O 2}$ is the concentration of monomeric silica at the equilibrium expressed as $\mathrm{SiO}_{2}$ in $\mathrm{mg} \mathrm{L}^{-1}$, [ $\left.\mathrm{Si}\right]$ is the concentration reported by the spectrophotometer for $\mathrm{Si}$ in $\mathrm{mg} \mathrm{L}^{-1}, A_{r}(\mathrm{Si})$ is the relative atomic mass of silicon. The equation is multiplied by 10 which is the dilution factor arising from the dilution of $0.4 \mathrm{ml}$ of sample in a total volume of $4 \mathrm{ml}$. 


\subsubsection{Measurement of the $\mathrm{pH}$ at the equilibrium}

From the $\mathrm{pH}$ point of view the reaction was considered to be in equilibrium when the value of it remained constant for a time longer than $4 \mathrm{~h}$. Refer to Section 2.7.1.4 for a description of the experimental setup.

\subsubsection{Mining waste characterisation}

\subsubsection{Characterisation of Suspended Solids}

A $50 \mathrm{~mL}$ aliquot of the liquid weak acid sample containing suspended solids was allowed to settle under ambient conditions over night. The supernatant liquid was removed with a syringe. A small amount of liquid was left behind covering the surface of the solids. These wetted solids at the bottom of the sample container were scraped out with a spatula and placed on a watch glass and dried at $383 \mathrm{~K}$ for $24 \mathrm{~h}$.

The solid was homogenized and used for different analytical techniques. A portion was analysed using a JEOL 6500-F Scanning Electron Microscope (SEM), equipped with Energy Dispersive Spectroscopy (EDS) and backscattered electron detector.

Another portion was studied using a X'PERT PRO PANALYTICAL X-Ray Diffractometer equipped with anode material $\mathrm{Cu}, \lambda=1.54060 \AA$ as a radiation source. The X-Ray Diffraction (XRD) pattern was obtained and compared against reference patterns from the powder diffraction file, version 4+, from International Centre for Diffraction Data, ICDD, Pennsylvania, USA.

A small portion of $0.1822 \mathrm{~g}$ of the suspended solids was digested in aqua regia and analysed using spiked samples method with Inductive Coupled Plasma Optical Emission Spectroscopy (ICP-OES) and Flame Atomic Absorption Spectroscopy (FAAS) in Nuremberg, Germany at the Georg-Simon-Ohm Hochschule and Victoria University of Wellington, New Zealand, respectively. The instruments used were Thermofisher ICAP-6200 and GBC 906, respectively. The analysed elements were: $\mathrm{Al}, \mathrm{As}, \mathrm{Ba}, \mathrm{Bi}, \mathrm{Ca}, \mathrm{Co}, \mathrm{Cr}, \mathrm{Cu}, \mathrm{Fe}, \mathrm{Hg}, \mathrm{K}, \mathrm{Mg}, \mathrm{Mn}, \mathrm{Mo}, \mathrm{Na}, \mathrm{Nb}, \mathrm{Pb}, \mathrm{Re}, \mathrm{Sb}, \mathrm{Si}, \mathrm{Sn}$, $\mathrm{Ti}, \mathrm{V}, \mathrm{Y}, \mathrm{Zn}$ and $\mathrm{Zr}$. These analytes were chosen in view of their economic importance, environmental hazard and probability of being present in the sample considering it was produced during copper processing.

\subsubsection{Liquid sample characterisation}

The following analytes were chosen taking in consideration the probability of species being present in the sample based on an analysis of the original ore, environmental concerns and potential recovery applications: $\mathrm{Al}, \mathrm{As}, \mathrm{Ca}, \mathrm{Co}, \mathrm{Cr}, \mathrm{Cu}, \mathrm{Fe}, \mathrm{K}, \mathrm{Mg}, \mathrm{Mo}$, 
$\mathrm{Na}, \mathrm{Ni}, \mathrm{Pb}, \mathrm{V}, \mathrm{Cl}^{-}, \mathrm{NO}_{3}{ }^{-}, \mathrm{SO}_{4}{ }^{2-}$, acidity, chemical oxygen demand (COD), conductivity and $\mathrm{pH}$. A copy of the result analysis can be viewed on A-18. The content of dissolved As, $\mathrm{SO}_{4}{ }^{2-}$ and \%wt suspended solids were provided directly via e-mail from the process engineer in charge of the operation of the sulfuric acid production plant at El Teniente.

\subsubsection{Characterisation of solid samples collected from kinetic and equilibrium studies of the adsorption of $\mathrm{Cu}^{2+}$ onto NCaSil}

Following are the procedure and techniques used in this thesis to characterize the solid sample obtained as described in sections 2.1 to 2.6.

\subsubsection{Characterisation with Scanning Electron Microscopy.}

Filter cakes were dried in air for $72 \mathrm{~h}$ and then ground in a mortar. A small portion was placed on a round SEM sample holder over carbon tape. Samples were coated with platinum and carbon to assure low charging while using the instrument. After being coated, samples were stored under high vacuum for $24 \mathrm{~h}$ before introducing them into the SEM. Micrographs and EDS mapping were obtain using a JEOL JSM 6500F field emission scanning electron microscope equipped with a backscatter detector. The energy of the electron beam was, in most cases, $15 \mathrm{kV}$.

SEM images correspond to an uptake experiment performed at $313 \mathrm{~K}$ as the author considered this a representative temperature to study. Different zones of each sample were scanned and the most representative ones were chosen in order to show the overall development of the reaction. Images were taken using the same magnification $5000 \mathrm{x}$, with the scale bar in the legend being $1 \mu \mathrm{m}$ to make comparison of images easier. The reaction consisted of $1 \mathrm{~g}$ of NCaSil contacted with $1 \mathrm{~L}$ of $15.7 \mathrm{mmol} \mathrm{L}^{-1}$ of $\mathrm{Cu}^{2+}$.

\subsubsection{Characterisation with powder X-Ray Diffraction}

Powder-XRD diffraction patterns were obtained using either a Phillips PW 3710 MPD controlled diffractometer, or a X'PERT PRO PANALYTICAL X-Ray Diffractometer Scans were recorded through an angular range of $4-70^{\circ} 2 \theta$. Different scan speeds were used with the most common being $1.1^{\circ} 2 \theta \mathrm{s}^{-1}$ for the Phillips instrument and $9.5^{\circ} 2 \theta \mathrm{s}^{-1}$ for the PANALYTICAL instrument. Even though the latter instrument was used at a faster scan speed the more modern detector gave a considerably better signal to noise ratio. Both instruments used the $\mathrm{Cu} \mathrm{K}_{\alpha}$ spectral line, $\lambda=1.54060 \AA$. Powder-XRD patterns were assigned to specific crystalline phases, taking reference patterns from the ICDD Powder Diffraction File Version 4 
and were of either Star or Indexed quality. The background was corrected in the Highexpert score software using a bending factor of 4 and granularity of 23 as presented on default setup. The intensity of peaks in the experimental XRD pattern was standardized using the highest peak as reference. Where $I$ is the intensity of any given data point on the pattern in counts, $I_{0}$ is the highest peak intensity in counts.

\subsection{Kinetic study using powder-X-Ray Diffraction.}

The filter cakes collected during kinetic experiments were dried in air for 72 hours. The powder was then placed in a custom built sample holder. A total number of fourteen sample holders were manufactured in order to maximize productivity and measure in the shortest time frame possible.

XRD patterns were collected and treated in a similar manner to section 2.7.5.2. Taking into consideration that the integrated peak area is proportional to the concentration of a given reflection in the crystal, it is possible to treat the experimental data in a similar way to adsorption data. In this case counts were used instead of normalized data. The integrated peak area given by the higher score software, in units of counts. $2 \theta$, was used without further changes. The variation of the integrated peak area with time was plotted in order to study the crystal growth. Pseudo first and pseudo second order models were used in order to retrieve kinetic data. 


\subsection{Statistical analysis and calculation of errors of the experimental data.}

The total uncertainties for the different methods were calculated using an up to date reference for quality control in chemical laboratories $[54,55]$. Is important to notice that glass expansion and contraction due to temperature were not considered as source of error since the laboratory had few episodes of large temperature variation.

Determining the uncertainty of a measurement allows comparison between laboratories and helps the user to evaluate the quality of his results. Experimental results are commonly expressed as:

$$
\mu \pm k \cdot \sigma
$$

Where $\mu$ is the average, $k$ is the coverage factor given by the confidence interval of the distribution of the results and $\sigma$ is the standard deviation of the dataset, also known as error or uncertainty. Knowing this, there are three common ways of expressing the uncertainty: first, it may be expressed as uncertainty (U), which corresponds to the standard deviation for a set of data $(\sigma)$ : second, the relative uncertainty $U_{r}$ which represents a portion of a certain standard value $X(\sigma / X)$; and third, as an expanded uncertainty, $\mathrm{U}_{\mathrm{ex}}$, which considers a coverage factor due to a confidence interval of the distribution of the data $(k \cdot \sigma)$. $\mathrm{U}_{\mathrm{r}}$ is useful as it allows adding different sources of errors that possess different units.

Usually manufacturers of instruments, equipment and standards provide the error as an expanded uncertainty. Therefore it is important to know whether $k$ has already been included to the reported error. It is common to find standards where errors are reported with a coverage factor of $1.96 \approx 2$, which is related to $95 \%$ confidence interval of the distribution.

To calculate the total uncertainty it is necessary to convert all the uncertainties (U) to relative uncertainties $\left(\mathrm{U}_{\mathrm{r}}\right)$.

Prior to calculations of a total uncertainty it is necessary to perform the following conversions:

1) Divide the expanded uncertainty $\left(\mathrm{U}_{\mathrm{ex}}\right)$ by the coverage factor $(k)$ reported by the manufacturer to obtain U. In case that the coverage factor is not known one can divide by $\sqrt{3}$ and $\sqrt{6}$ assuming a rectangular distribution or a 
triangular distribution respectively. In a triangular distribution the mean value has a higher probability of occurrence, while in the rectangular distribution all values have the same probability.

2) Divide the $U$ by the standard value reported in the manufacturer to obtain $U_{r}$.

Followed is an example of the calculation of the relative uncertainty of a $\mathrm{Cu}$ standard for FAAS and ICP measurement.

Example: Calculation of the $\mathrm{U}_{\mathrm{r}}$ of the $\mathrm{Cu}$ standard.

The manufacturer, Flukka, reports a concentration of $1000 \pm 2$, with a $95 \%$ confidence interval $(k=2)$.

Therefore

$U_{e x}=2 \frac{m g}{L}$

In order to obtain the uncertainty, one must divide $\mathrm{U}_{\mathrm{ex}}$ by the corresponding coverage factor,

$U=\frac{2}{2}=1 \frac{m g}{L}$

Subsequently by diving $U$ by the standard value reported by the manufacturer the relative uncertainty is obtained,

$U_{r}=\frac{1}{1000}=1 \times 10^{-3}$ 
In order to calculate the total uncertainty for a calibration curve built using copper standards, the different sources of error were identified. Figure 2.7 shows a diagram depicting the sources of error during the preparation of the calibration curve.

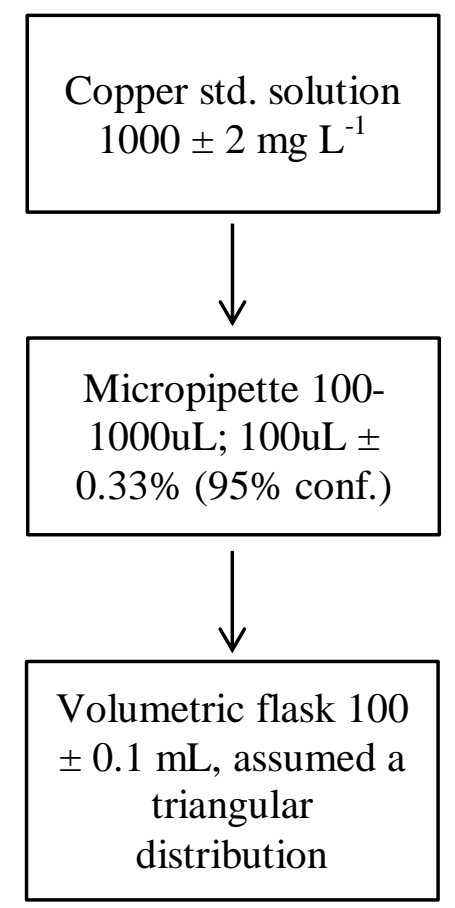

Figure 2.7: Error contributions for the preparation of a $\mathrm{Cu}^{2+}$ calibration curve.

Calculation of total uncertainty in the preparation of a copper calibration curve according to the contributions shown in Figure 2.7:

Uncertainties:

- Copper standard solution: $U=\frac{2}{2}=1 \frac{m g}{L} ; U_{r}=\frac{1}{1000}=1 \cdot 10^{-3}$

- $100-1000 \mu \mathrm{L}$ micropipette: $U=\frac{\frac{0.33 \cdot 100}{100}}{2}=0.17 \mu \mathrm{L}$;

$$
U_{r}=\frac{0.17}{100}=3.3 \cdot 10^{-3} \mu L
$$

- $100 \mathrm{~mL}$ volumetric flask: $U=\frac{0.1}{\sqrt{6}}=0.41 \mathrm{~mL} ; U_{r}=\frac{0.41}{100}=4.1 \cdot 10^{-3}$

The total relative uncertainty is calculated by the following expression:

$$
U_{T, r}=\sqrt{\left(U_{r(1)}\right)^{2}+\left(U_{r(2))}\right)^{2}+\ldots+\left(U_{r(n)}\right)^{2}}
$$

Where $\mathrm{U}_{T, r}$ is the total relative uncertainty and $\mathrm{U}_{\mathrm{r}(1)}$ to $\mathrm{U}_{\mathrm{r}(\mathrm{n})}$ correspond to the contribution of all sources of error. 
Consequently, the value of the total uncertainty for the preparation of the calibration curve is given by:

$$
\begin{aligned}
& U_{T, r}=\sqrt{\left(1 \cdot 10^{-3}\right)^{2}+\left(4.1 \cdot 10^{-3}\right)^{2}+\left(3.3 \cdot 10^{-3}\right)^{2}} \\
& U_{T, r}=5.3 \cdot 10^{-3}
\end{aligned}
$$

The value $U_{T, r}$ may now be multiplied by a coverage factor giving the total expanded uncertainty $\left(\mathrm{U}_{\mathrm{T}, \mathrm{ex}}\right)$. $\mathrm{U}_{\mathrm{T}, \mathrm{ex}}$ may be multiplied by 100 to express it as a percentage.

Next is an example of how the $\mathrm{U}_{\mathrm{T}, \mathrm{ex}}$ was obtained for the uptake of $\mathrm{Cu}^{2+}$ by $\mathrm{NCaSil}$ as described in Section 2.3.2 


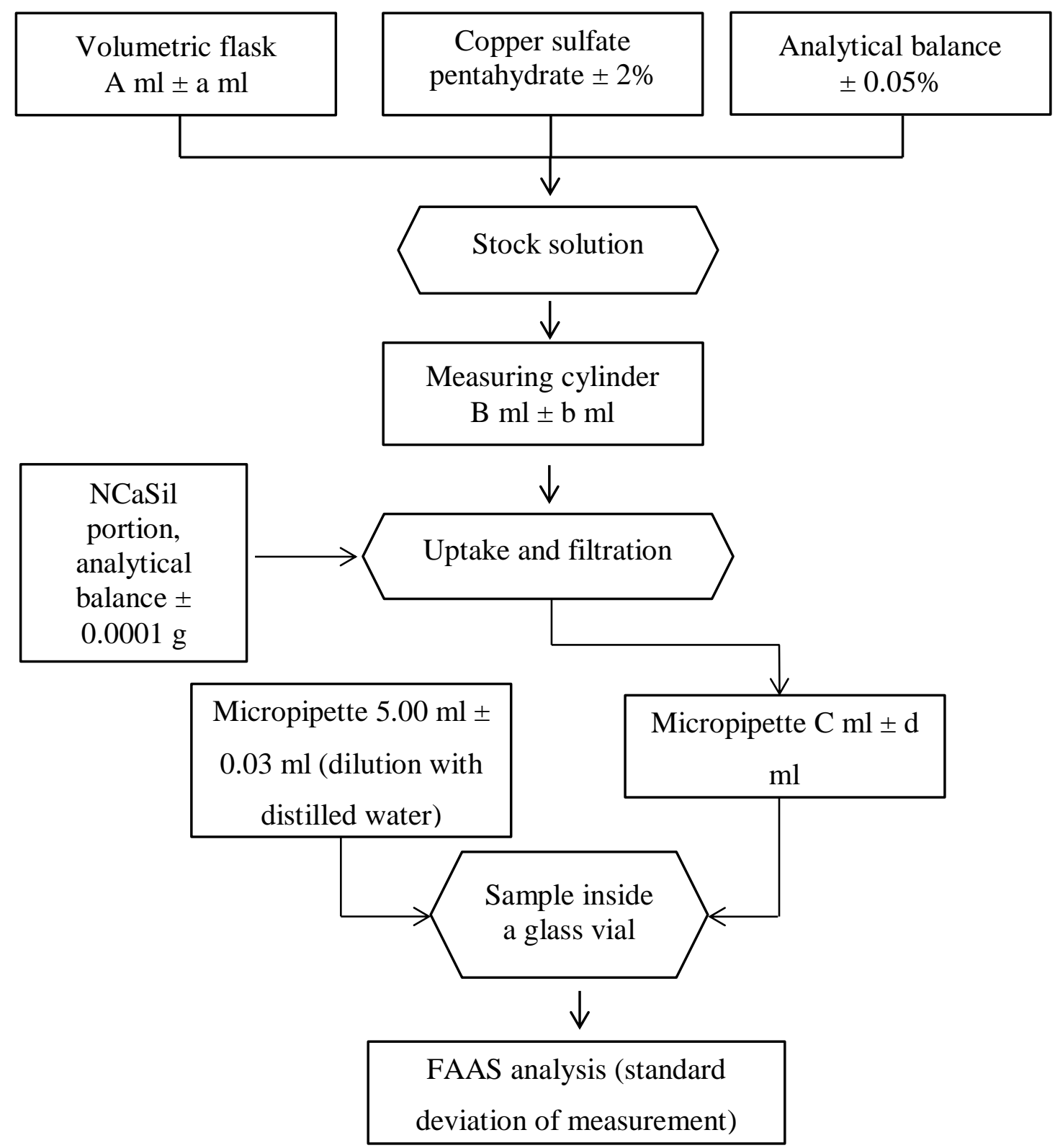

Figure 2.8: Error contribution for kinetic experiments done as described in Section 2.3.2 and 2.3.4.1

Figure 2.8 shows the entire process from the preparation of the stock solution until the measurement on the FAAS. Error contribution steps are shown in square shaped boxes, while diamond shaped boxes represent steps or actions during the experiment that are not considered to have error contribution.

Considering the common dilutions, volumetric flasks, micropipettes and concentrations used for the experiments the following magnitudes for the relative uncertainties were calculated: 
Uncertainty source:

- $\quad$ Copper sulfate pentahydrate: $U_{r}=2 \cdot 10^{-2}$

- 1 L volumetric flask: $U=\frac{0.1}{\sqrt{6}}=0.41 m L ; U_{r}=\frac{0.41}{1000}=4.1 \cdot 10^{-3}$

- Analytical balance: $U_{r}=5 \cdot 10^{-4}$

- Measuring cylinder $1 \mathrm{~L}: U=\frac{0.5}{\sqrt{6}}=0.371 \mathrm{~mL} ; U_{r}=\frac{0.371}{1000}=3.71 \cdot 10^{-4}$

- Micropipette $5.00 \mathrm{ml}: U_{r}=5 \cdot 10^{-3}$

- Micropipette 50-250 $\mu \mathrm{L}: U_{r}=5 \cdot 10^{-3}$

- FAAS calibration curve: $U_{r}=5.3 \cdot 10^{-3}$

- FAAS measurement (typical relative uncertainty when a sample is measured by triplicate): $U_{r}=1 \cdot 10^{-2}$

The total relative uncertainty is calculated by the following expression:

$U_{T, r}=\sqrt{\left(U_{r(1)}\right)^{2}+\left(U_{r(2))}\right)^{2}+\ldots+\left(U_{r(n)}\right)^{2}}$

Where $\mathrm{U}_{T, r}$ is the total relative uncertainty and $\mathrm{U}_{\mathrm{r}(1)}$ to $\mathrm{U}_{\mathrm{r}(\mathrm{n})}$ corresponds to the contribution of all sources of error.

Consequently, the value of the total uncertainty for the preparation of the calibration curve is given by:

$U_{T, r}=\sqrt{\left(2 \cdot 10^{-2}\right)^{2}+\left(4.1 \cdot 10^{-3}\right)^{2}+2 \cdot\left(5 \cdot 10^{-4}\right)^{2}+\left(3.71 \cdot 10^{-4}\right)^{2}+2 \cdot\left(5 \cdot 10^{-3}\right)^{2}+\left(5.3 \cdot 10^{-3}\right)^{2}+\left(1 \cdot 10^{-2}\right)^{2}}$

$U_{T, r}=2.4 \cdot 10^{-2}$

The total uncertainty for a kinetic experiment is $2.4 \%$. The expanded total uncertainty $\left(\mathrm{U}_{\mathrm{T}, \mathrm{ex}}\right)$ with a $95 \%$ reliability is the reported value multiplied by $\mathrm{k}=1.96$ $\approx 2$, giving $\mathrm{U}_{\mathrm{T}, \mathrm{ex}} 4.8 \%$. The largest contribution to the methods uncertainty comes from the copper sulfate and the FAAS instrument with 2.0 and $1.0 \%$ respectively. 
The total expanded uncertainty was calculated for each method using the same method as above. Table 2.3 summarizes the total expanded uncertainty for all carried experiments.

Table 2.3: Summary of total expanded uncertainty

\section{Experiment}

\section{Total Expanded} Uncertainty

( $\mathrm{U}_{\mathrm{T}, \mathrm{ex}} ; 95 \%$ conf. int.)
$\mathrm{Cu}^{2+}$ sorption

(Kinetic)

$\mathrm{Cu}^{2+}$ sorption

(Equilibrium Procedure A)

$\mathrm{Cu}^{2+}$ sorption

(Equilibrium Procedure B)

$\mathrm{Cu}^{2+}$ continuous sorption

(conventional axial flow column

and radial flow column)

$\mathrm{Ca}^{2+}$ release

(Kinetic, equilibrium and continuous sorption studies)

$\mathrm{SiO}_{4}{ }^{4-}$ release

(Kinetic, equilibrium, continuous sorption studies)
2.2 and 2.7.1

$4.8 \%$

2.3 and 2.7 .2

$4.8 \%$

2.3 and 2.7 .2

$4.8 \%$

2.5 and 2.7 .1

$4.7 \%$

$2.2,2.3,2.7 .1$ and 2.7.2

$6.5 \%$

$2.2,2.3,2.7 .1$ and

2.7.2

$3.2 \%$

2.7.1 and 2.7.2

$0.7 \%$ 


\section{Chapter 3 , A study of the adsorption of $\mathrm{Cu}^{2+}$ ions onto NCaSil}

Kinetic and equilibrium studies regarding the adsorption of $\mathrm{Cu}^{2+}$ onto $\mathrm{NCaSil}$ were carried out at different temperatures ranging from 277 to $343 \mathrm{~K}$. Rate constants and equilibrium parameters were evaluated and utilized to calculate thermodynamic parameters such as the activation energy $\left(E_{a}\right)$, Gibbs free energy $(\Delta G)$, standard enthalpy $\left(\Delta H^{\circ}\right)$, and standard entropy $\left(\Delta S^{\circ}\right)$ of the sorption process. A follow-up study of the uptake of copper and crystal development of copper sulfate hydroxide compounds on the surface on NCaSil was done using SEM, EDS and powder-XRD seeking to understand its dependence, to the initial concentration of $\mathrm{Cu}^{2+}$ in solution, time and temperature.

\subsection{Introduction to adsorption}

Adsorption is a process where ions and/or molecules interact physically with or bind chemically to the surface of an adsorbent. Physisorption mainly involves van der Waals interactions which are weak, while in chemisorption they attach to the surface through a process which involves formation of bonds.

The adsorption of an adsorbate $A$ in solution onto a adsorbent $M$ can be described by the following equation:

$A_{(a q)}+M_{(S)} \leftrightharpoons A M_{(S)}$

Even though eq. 3.1 seems simple, the adsorption process may involve several steps before adsorbate $A$ settles on the surface. Commonly the mechanism by which adsorbate 
$A$ approaches and further reacts/interacts with the surface of the adsorbent can be summarized in the following steps and is shown in Figure 3.1.

1. The adsorbate $A$ diffuses from the bulk of the solution to a static film, also called boundary layer, surrounding the adsorbent particle.

2. The adsorbate $A$ diffuses through the static film until it reaches the surface.

3. If the adsorbent is porous the adsorbate $A$ will enter through the pores via intraparticle diffusion.

4. The adsorbate $A$ settles on an available site by a physical or a chemical interaction.

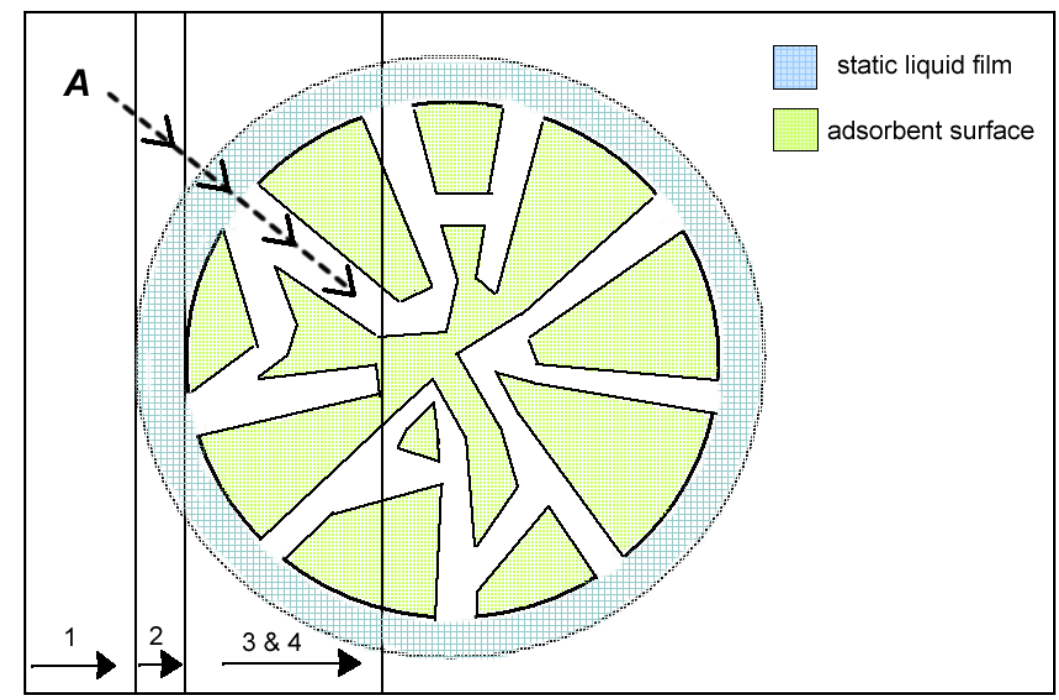

Figure 3.1: General sorption mechanism onto a spherical porous solid.

If the process is under mechanical stirring the diffusion from the bulk of the solution of $A$ (step 1) can be neglected. Also the thickness of the static liquid film surrounding the adsorbent particle can be diminished by increasing the stirring speed making it possible to neglect the diffusion through the static liquid film (step 2) in some cases.

\subsubsection{Sorption Kinetics}

As early as 1898 kinetics of the adsorption of adsorbates in solution onto a solid were studied for the sorption of oxalic-manolic acid onto charcoal by Lagergren [56]. Several kinetic models have been developed since then, including the Elovich equation derived from the work of Zeldowitsch in 1934 on the sorption of carbon monoxide onto manganese dioxide [57]. In order to distinguish these models from standard chemical kinetics the literature refers to them as pseudo-order models [57], despite the fact that this term is widely used in physical chemistry for orders different from an integer $\geq 0$. 
Adsorbate concentration on the surface of the solid is expected to increase with decreasing rate over time as sites on the adsorbent become occupied. Hence the rate of the reaction will follow the form:

$$
\frac{d x}{d t}=k\left(x_{e q}-x\right)^{n}
$$

Where $k$ is the rate constant, $x_{\text {eq }}$ is the concentration of the adsorbate on the surface of the adsorbent as $t \rightarrow \infty$ and $n$ is the exponent that in the case of fitting to kinetic curves will be the order of the reaction, normally related to the reaction mechanism (e.g. unimolecular, bimolecular, etc...). As in standard chemical kinetics the order of the model reflects the way that the concentration changes over time. For example, in a firstorder kinetic expression the exponential in the rate expression is 1 in respect to the concentrations of the reagents. In pseudo-first-order models the exponential is also 1 but in respect to the available sites remaining. Often the kinetic relation is plotted as the amount of adsorbate per mass of adsorbent (e.g. $\mathrm{mmol} \mathrm{g}^{-1}$ ) as a function of time. If the experimental data is modelled using these equations the rate constant may be obtained.

Ho [57] states that pseudo-first order kinetic models are more likely to fit adsorption processes describing the uptake of adsorbate from solution onto a solid adsorbent in which the rate-limiting step is diffusion (eg. intraparticle, film diffusion), while pseudosecond order kinetics models are more likely to fit processes in which the rate-limiting step is the chemisorption of the involved species.

\subsubsection{Pseudo-first order}

Pseudo-first order equations have been widely used for obtaining kinetic parameters in the literature [57, 58]. The Lagergren equation is the first known mathematical description of sorption kinetics. The rate equation is given by,

$\frac{d q_{t}}{d t}=k\left(q_{e}-q_{t}\right)$

where $t$ is the time in $\min , q_{e}$ and $q_{t}$ are in $\mathrm{mmol} \mathrm{g}^{-1}$ and represent the sorption capacities at the equilibrium and time $t, k$ is the rate constant in $\min ^{-1}$. The rate of sorption is proportional to a constant and the amount of available sites left.

Integrating eq. 3.3 between the boundary conditions $t=0$ to $t=t$ and $q_{t}=0$ to $q_{t}=q_{t}$ yields,

$q_{t}=q_{e}\left(1-\mathrm{e}^{(-k t)}\right)$ 
or in the linear form,

$\log \left(q_{e}-q_{t}\right)=\log \left(q_{e}\right)-\frac{k}{2.306} t$

By plotting $\log \left(q_{e}-q_{t}\right)$ as a function of $t$, the kinetic rate constant and the sorption capacity at the equilibrium for the given experimental conditions may be obtained from the slope and intercept, respectively.

\subsubsection{Pseudo-second order}

A pseudo-second order model was first introduced by Blanchard in 1984 [30] which described the sorption of divalent metal ions onto a zeolite by an ion exchange mechanism. Where the zeolite exchanged 2 moles of ammonium ions for 1 mole of divalent metals as shown in eq. 3.6.

$Z_{\left(2 N H_{4}^{+}\right)}+M^{2+} \rightarrow Z_{\left(M^{2+}\right)}+2 N H_{4}^{+}$

Blanchard represented this process as a differential equation that depicts the rate of sorption as being proportional to a rate constant and to the square of the remaining unoccupied sites:

$\frac{d q_{t}}{d t}=-k\left(q_{e}-q_{t}\right)^{2}$

where, $t$ is the time in min, $q_{t}$ is the amount adsorbed at the time $t$ in $\mathrm{mmol} \mathrm{g}^{-1}, k$ is the kinetic constant in $\mathrm{g} \mathrm{mmol}^{-1} \mathrm{~min}^{-1}$ and $q_{e}$ is a constant that represents the amount adsorbed at the equilibrium in mmol $\mathrm{g}^{-1}$. In 1999 McKay and Ho [59] presented a pseudo-second order model based on Blanchard's differential equation and gave further meaning to the constants. By integrating between the boundaries $t=0$ to $t=t$ and $q_{t}=0$ and $q_{t}=q_{t}$ and re-arranging, eq. 3.8 is obtained.

$q_{t}=\frac{k q_{e}^{2} t}{\left(1+k q_{e} t\right)}$

Rearranging eq. 3.8 to a linear form, eq. 3.9 is obtained,

$\frac{t}{q_{t}}=\frac{1}{k q_{e}^{2}}+\frac{1}{q_{e}} t$

Plotting $t / q_{t}$ against $t$, allows $q_{e}$ and $k$ to be obtained from the slope and intercept respectively. In this model $k q_{e}{ }^{2}$ is often written as $h$ and is the initial sorption speed in mmol $\mathrm{g}^{-1} \min ^{-1}$. This model has been successfully used to model the sorption of 
compounds in aqueous solutions onto solid adsorbents in various publications $[3,57$, 59].

\subsubsection{Activation energy}

The kinetic rate constants obtained by the pseudo order models shown in this section can be used to obtain the activation energy $\left(E_{a}\right)$ of the process through an Arrehnius plot represented by eq. 3.10 [60].

$\ln k=\ln A-\frac{E_{a}}{R T}$

where $k$ is the kinetic rate constant obtained by any of the kinetic models, discussed in Section $4, E_{a}$ is the activation energy in $\mathrm{J} \mathrm{mol}^{-1}, A$ is a correlation factor, $R$ is the gas constant $8.314 \mathrm{~J} \mathrm{~mol}^{-1} \mathrm{~K}^{-1}$ and $T$ is the temperature in $\mathrm{K}$. By plotting $\ln k$ as a function of $\mathrm{T}^{-1}$ the value of $E_{a}$ can be obtained from the slope. $E_{a}$ is important since it suggests if the nature of the sorption is either physical or chemical. The activation energy for physical adsorption mechanisms are reported to be in the range of 5 to $30 \mathrm{~kJ} \mathrm{~mol}^{-1}$, while chemical adsorption mechanisms are commonly between $40-800 \mathrm{~kJ} \mathrm{~mol}^{-1}$ [61]. The value obtained by this mathematical analysis gives a suggestion for what the nature of the process could be, since in the adsorption process many steps are involved and the value may change with the adsorbent and adsorbate being used. 


\subsection{Results and discussion for the adsorption kinetic of $\mathrm{Cu}^{2+}$ onto NCaSil}

The uptake kinetics of $\mathrm{Cu}^{2+}$ ions onto NCaSil was studied at different temperatures. The solid material generated from these studies was characterised by means of powderXRD, SEM and EDS. These studies were performed in order to understand the mechanism by which crystals are formed and clarify whether NCaSil works as a substrate for crystal growth or crystals were formed in solution as a result of $\mathrm{OH}^{-}$being released from $\mathrm{NCaSil}$ into the solution over time.

\subsubsection{The effect of stirring on the kinetics of the adsorption}

Mass transfer refers to the movement of material in a certain space. In the case of the adsorption of species from solution this is related to the movement of the adsorbate from the bulk of the solution onto the surface of the adsorbent. In solution the movement of species is considered to occur by three mechanisms [62]:

1- Migration: charged species move due to a potential gradient.

2- Convection: species in solution move due to mechanical forces (e.g. stirring, pressure).

3- Diffusion: species in solution move due to a concentration gradient.

As discussed in Sections 3.1 and 3.2 stirring the solution inside a batch reactor improves mass transfer from the bulk of the solution onto the boundary layer of a solid particle. The magnitude of the concentration gradient between the bulk and the static film surrounding the particle becomes closer to zero. Hence, diffusion from the bulk to the particle's outskirt should become a less predominant mechanism in the adsorption. Furthermore, increasing the stirring speed makes the static film surrounding a particle thinner which aids mass transfer onto and into the solid.

Doing experiments keeping all variables constant but changing the stirring speed will show to what extent film diffusion is the controlling step of the adsorption. A $15.7 \mathrm{mmol} \mathrm{L}^{-1}$ solution was contacted with $1 \mathrm{~g}$ NCaSil using stirring speeds of 50 and $500 \mathrm{rpm}$. This large difference in stirring speed is necessary since it has been reported in the literature that mild changes in kinetics are observed in the range of 50-200 rpm [63]. Figure 3.2 shows the results for experiments carried out at different stirring speeds. 


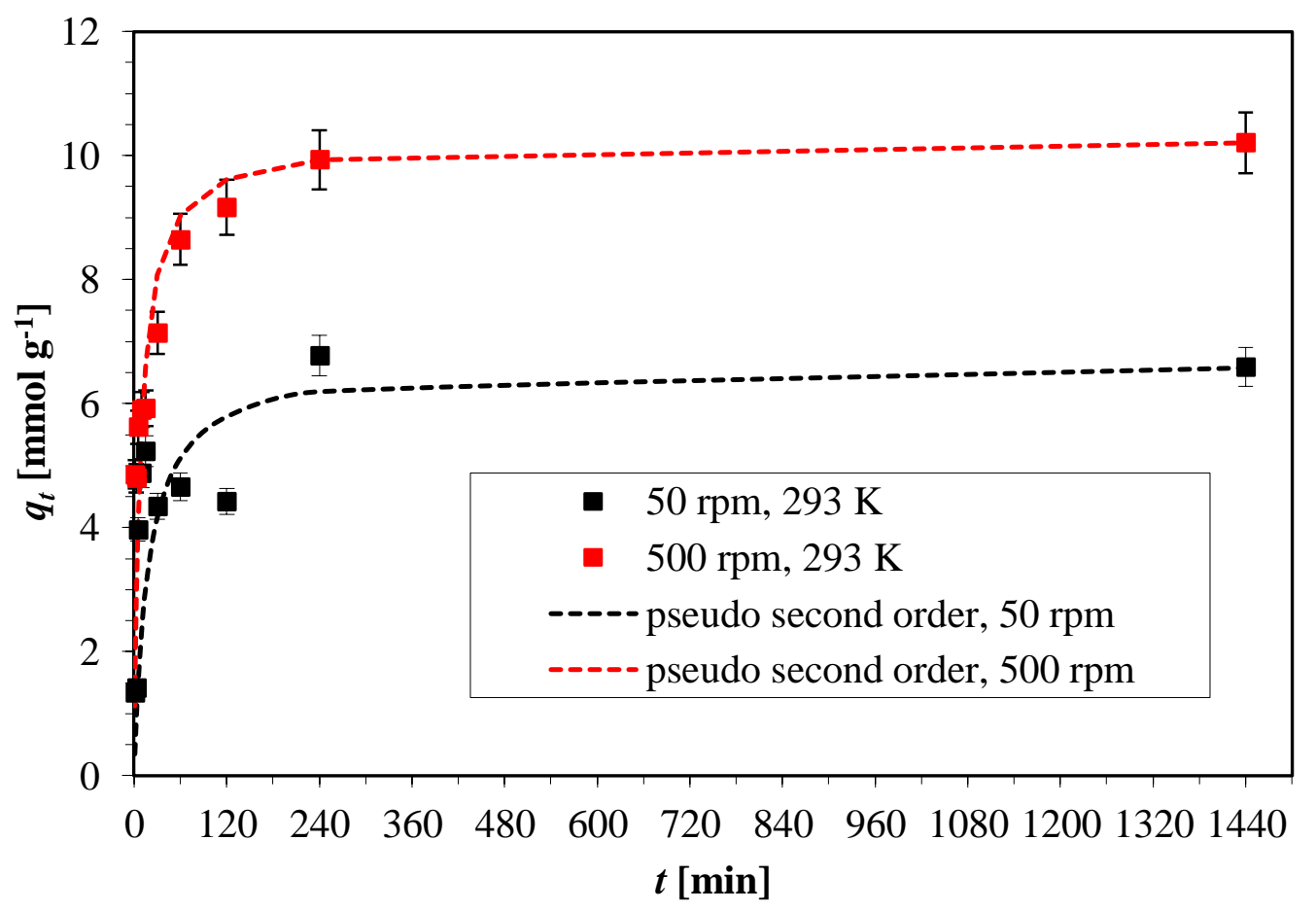

Figure 3.2: Kinetic study with different stirring speeds at 293 K. Experimental conditions adsorbent dosage $1 \mathrm{~g} \mathrm{~L}^{-1} ;\left[\mathrm{Cu}^{2+}\right]_{\text {initial }} 15.7 \mathrm{mmol} \mathrm{L}^{-1}$.

In Figure 3.2 the improvement on the uptake capacity of the adsorbent is clearly observed as the stirring speed is increased tenfold. This could be attributed to film diffusion being the limiting step of the mass transport to the surface or due to partial disaggregation of the NCaSil aggregates in solution. Aggregation would prevent fresh surface coming into contact with the solution, thus reducing the adsorption. This is an important parameter to consider for the design of a continuous system since no agitation will be present; hence disaggregation of NCaSil lumps is unlikely to occur, decreasing adsorption capacity.

The experimental data was fitted to pseudo-first and pseudo-second order models using a linear regression as described in Section 3.1.1.1 and 3.1.1.2. The values of the kinetic parameters are summarized in Table 3.1. 
Table 3.1: Pseudo-second order model kinetic parameters for the uptake of $\mathrm{Cu}^{2+}$ with different stirring speed at $293 \mathrm{~K}$ for the plot presented in Figure 3.2.

\begin{tabular}{|c|c|c|c|c|}
\hline $\begin{array}{c}\text { Stirring } \\
\text { speed } \\
{\left[\text { min }^{-1}\right]}\end{array}$ & $\frac{k}{\left[\mathrm{~g} \mathrm{mmol}^{-1} \min ^{-1}\right]}$ & $\begin{array}{c}q_{\mathrm{e}} \\
{\left[\mathrm{mmol} \mathrm{g}^{-1}\right]}\end{array}$ & $\begin{array}{c}k q_{e}^{2} \\
{\left[\mathrm{mmol} \mathrm{g}^{-1} \min ^{-1}\right]}\end{array}$ & $r^{2}$ \\
\hline 50 & 0.011 & 6.6 & 0.5 & 0.9983 \\
\hline 500 & 0.012 & 10.3 & 1.3 & 0.9999 \\
\hline
\end{tabular}

Pseudo-first order models had a low correlation to the experimental data with values of $r^{2}<0.6571$. It is most likely that the kinetics obey a pseudo-second order model as values of $r^{2}$ larger than 0.9983 were obtained for both experiments. The value of $q_{e}$ was $43 \%$ higher when the stirring speed was increased. The initial adsorption speed $h\left(k q_{e}{ }^{2}\right)$ is two times faster when the faster stirring speed is used, due to dependence of $h$ to the square of $q_{\mathrm{e}}$. Experimental results show that a high stirring speed has to be used to guarantee maximum surface coverage. For this reason, all further batch experiments were undertaken at a stirring speed of $500 \mathrm{rpm}$.

3.2.2 The effect of temperature on the kinetic of the adsorption of $\mathrm{Cu}^{2+}$ onto NCaSil

Variation of the temperature will have a direct impact according to the Arrhenius equation. Figure 3.3 shows the experimental data obtained for the uptake of $\mathrm{Cu}^{2+}$ ions onto NCaSil for all studied temperatures. The uptake occurred rapidly with a clear increase in the rate of the sorption as the temperature rose. The equilibrium was reached between 60 and $240 \mathrm{~min}$, with an amount adsorbed at equilibrium ranging from 7.9 to $10.4 \mathrm{mmol} \mathrm{g}^{-1}$, depending on the temperature. After a few hours a change in colour could be observed, changing from blue to turquoise. This drift in colour occurred faster as the temperature increased, reducing from $20 \mathrm{~h}$ to $30 \mathrm{~min}$ when the temperature was raised from 293 to $343 \mathrm{~K}$. This phenomenon will be discussed later in the next section where XRD patterns of the loaded adsorbent are analysed. 


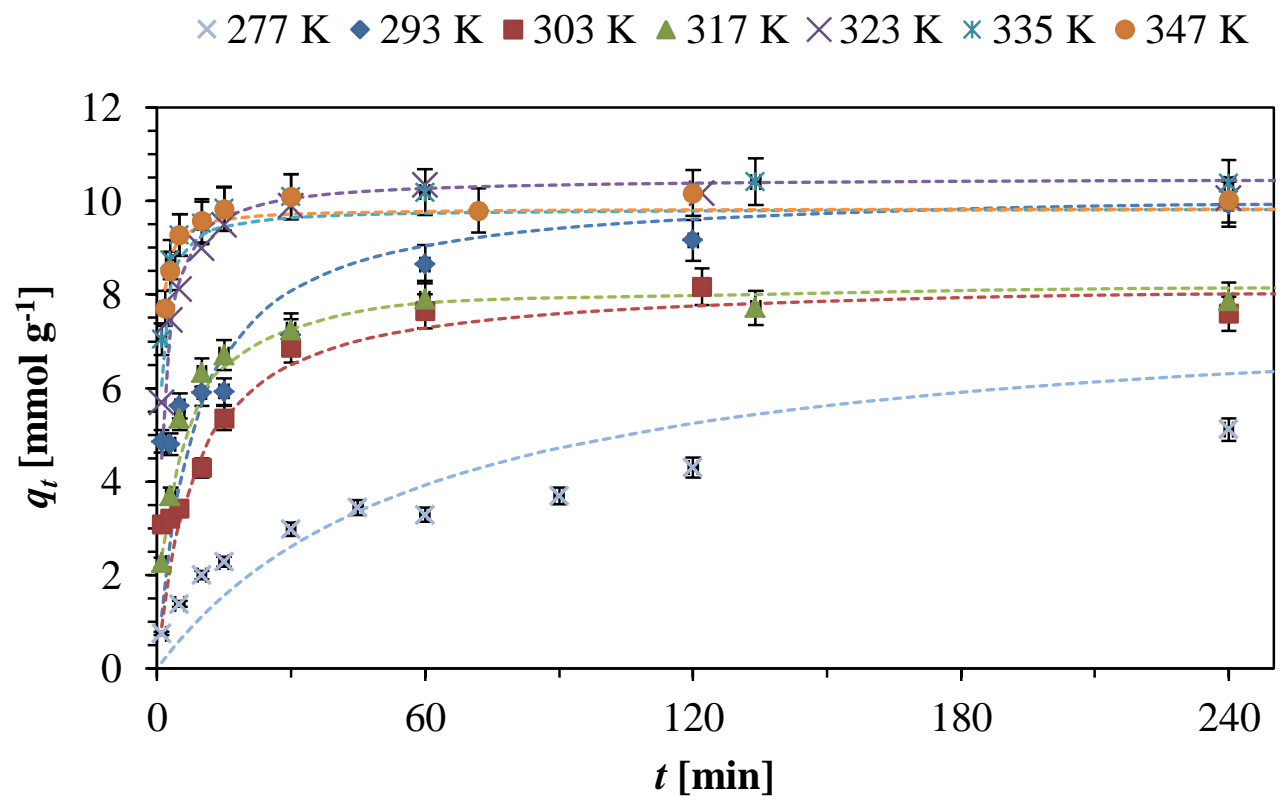

Figure 3.3: Experimental and modelled kinetic data for $\mathrm{Cu}^{2+}$ uptake onto $\mathrm{NCaSil}$ for temperatures ranging between 277 and $347 \mathrm{~K}$. Experimental conditions: adsorbent dosage $1 \mathrm{~g} \mathrm{~L}^{-1}$; [ $\left.\mathrm{Cu}^{2+}\right]_{\text {initial }}$ $15.7 \mathrm{mmol} \mathrm{L}^{-1}$.

To obtain kinetic parameters, pseudo-order models were fitted to the experimental data. Plots based on the pseudo-first order model presented a low correlation between the model and the experimental data $\left(r^{2}<0.8\right)$. The pseudo-second order model had a high correlation $\left(r^{2}>0.99\right)$ for all data sets, suggesting a chemical adsorption processes.

Using eq. 3.9 and evaluating both intercept and slope the values for the constants were obtained and listed in Table 3.2 for all the studied temperatures.

Table 3.2: Kinetic parameters for the pseudo-second order equation calculated from the data shown in Figure 3.3.

\begin{tabular}{|c|c|c|c|c|}
\hline $\begin{array}{c}\mathbf{T} \\
{[\mathbf{K}]}\end{array}$ & $\begin{array}{c}q_{\mathrm{e}} \\
{\left[\mathrm{mmol} \mathrm{g}^{-1}\right]}\end{array}$ & $\begin{array}{c}k \\
{\left[\mathrm{~g} \mathrm{mmol}^{-1} \mathrm{~min}^{-1}\right]}\end{array}$ & $\begin{array}{c}k q_{\mathrm{e}}^{2} \\
{\left[\mathrm{mmol} \mathrm{g}^{-1} \mathrm{~min}^{-1}\right]}\end{array}$ & $r^{2}$ \\
\hline 277 & 7.90 & 0.00210 & 0.130 & 0.9983 \\
\hline 293 & 9.20 & 0.0103 & 0.870 & 0.9969 \\
\hline 303 & 7.88 & 0.0296 & 1.84 & 0.9973 \\
\hline 317 & 7.89 & 0.0285 & 1.77 & 0.9974 \\
\hline 323 & 10.1 & 0.146 & 15.0 & 1.0000 \\
\hline 335 & 10.4 & 0.127 & 13.8 & 0.9999 \\
\hline 347 & 10.1 & 0.246 & 24.8 & 0.9999 \\
\hline
\end{tabular}

The pseudo-second order model has an outstanding fit with values of $r^{2}>0.9969$ for all the studied temperatures. It has been mentioned in the literature that processes, for which experimental data fit this model, are likely to involve a chemical reaction with 
the surface [57]. The plot of the modelled data is displayed in Figure 3.3 where a good correlation between the model and the experimental data is also observed.

The values of the kinetic rate constant $k$ and the initial adsorption rate $k q_{e}{ }^{2}$ increase as the temperature rises reaching a value of $0.246 \mathrm{~g} \mathrm{mmol}^{-1} \mathrm{~min}^{-1}$ and $24.8 \mathrm{mmol} \mathrm{g}^{-1} \mathrm{~min}^{-1}$ at $347 \mathrm{~K}$, respectively. This means the second order kinetic rate constant $k$ increases by 2 orders of magnitude when the temperature rises $70 \mathrm{~K}$. On the other hand, the value of $q_{e}$ tends to increase with temperature, reaching a maximum of approximately $10 \mathrm{mmol}$ $\mathrm{g}^{-1}$ at temperatures higher than $323 \mathrm{~K}$. Comparing these results to other silicate containing materials in the literature, NCaSil exhibits similar rate of adsorption but ten times larger loading capacities. For example, Apiratikul et al. [64] reported maximum loading capacities for copper of only $1.43 \mu \mathrm{mol} \mathrm{g}{ }^{-1}$ onto a modified zeolite derived from coal fly ash. The adsorption followed a pseudo-second order kinetic model with outstanding correlation between the model and the experimental data reaching equilibrium in 60 minutes for a concentration of $5 \mathrm{mmol} \mathrm{L}^{-1} \mathrm{Cu}^{2+}$. In a different work, Erdem et al. [21] obtained a maximum loading capacity by the Langmuir isotherm of $114 \mu \mathrm{mol} \mathrm{g}^{-1}$ for the adsorption of copper onto a natural zeolite from the Enli Mining Company open pit mine in Manisa-Gördes in Western Anatolia. It is clear that compared to these studies that NCaSil has a loading capacity of at least 2 orders magnitude higher than those reported for zeolites.

In order to obtain the activation energy of the sorption process in solution an Arrhenius [65] plot was generated using the kinetic rate constants shown in Table 3.2. Figure 3.4 displays such plot in the temperature range from $293 \mathrm{~K}$ to $343 \mathrm{~K}$. 


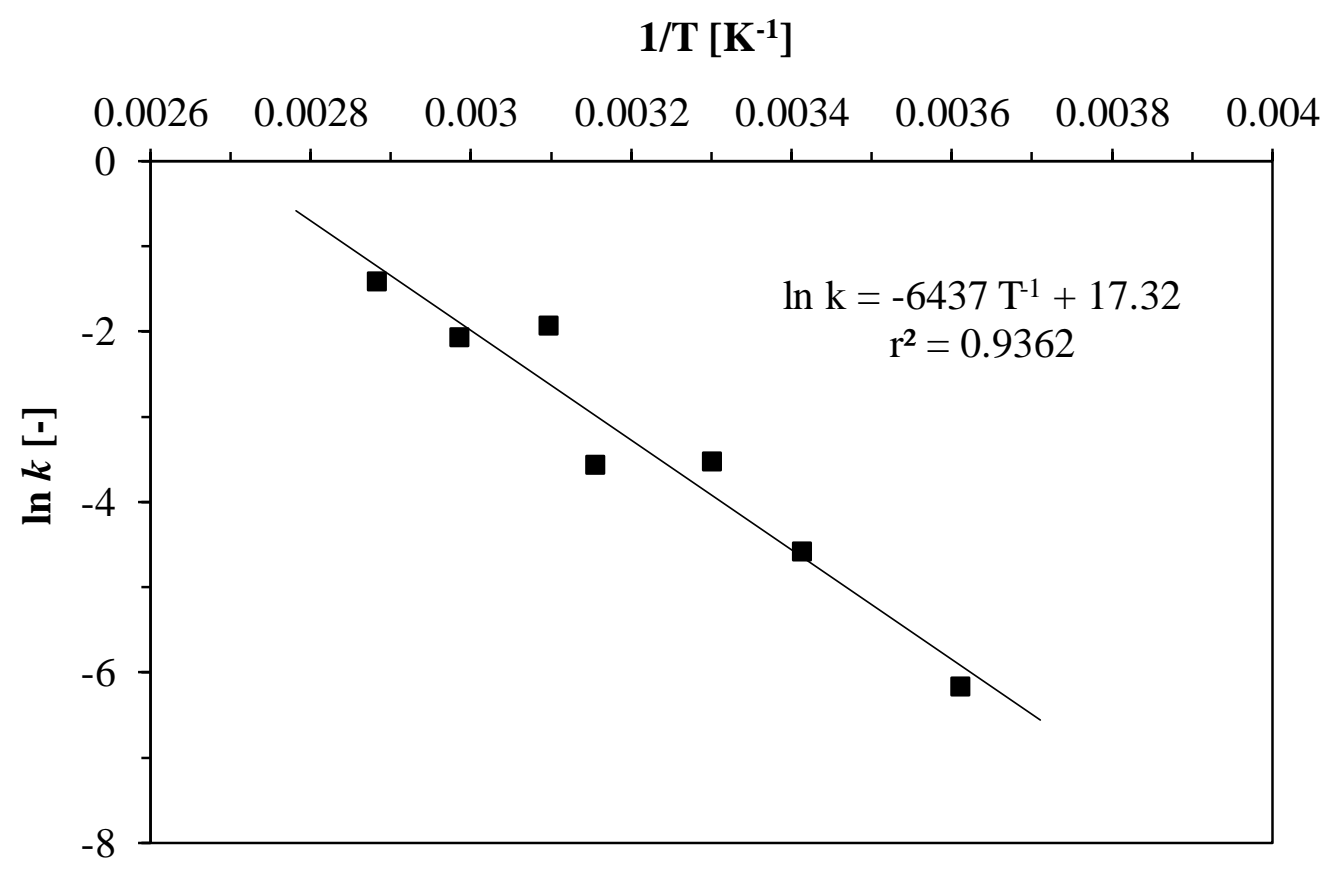

Figure 3.4: Arrhenius plot using the kinetic rate constant for $\mathrm{Cu}^{2+}$ uptake onto NCaSil shown in Table 3.2.

Figure 3.4 shows that the Arrhenius plot gives a reasonable correlation for the plotted data $\left(r^{2}=0.9362\right)$. In the literature, physical adsorption activation energies are in the range of $5-40 \mathrm{~kJ} \mathrm{~mol}^{-1}$, while chemical adsorption varies between 40 and $800 \mathrm{~kJ} \mathrm{~mol}^{-1}$ [66]. In this study a value for the activation energy of $54 \mathrm{~kJ} \mathrm{~mol}^{-1}$ was obtained, suggesting that the adsorption occurred via a chemical reaction with the surface. Furthermore, the possibility that the rate limiting step of the adsorption is diffusion can be discarded since energies for such processes are usually below $25-30 \mathrm{~kJ} \mathrm{~mol}^{-1}$ [3].

\subsubsection{Kinetic study at different temperatures on the release of calcium ions}

Figure 3.5 shows the release of $\mathrm{Ca}^{2+}$ ions when $1 \mathrm{~g}$ of $\mathrm{NCaSil}$ is contacted with a solution containing $15.7 \mathrm{mmol} \mathrm{L}^{-1}$ of $\mathrm{Cu}^{2+}$.

It is possible to use eq. 3.2 to model the kinetics of calcium and monomeric silica release in order to get the kinetic rate constant. To do so the rate expression should be rewritten as follows,

$$
\frac{d q_{r, t, C a}}{d t}=k\left(q_{r, e, C a}-q_{r, t, C a}\right)^{n}
$$

Where $k$ is the kinetic rate constant of an $n^{\text {th }}$ order model, $q_{r, e, C a}$ the concentration in solution when $t \rightarrow \infty$ and $q_{r, t, C a}$ is the concentration in solution at any time $t$.

Both reactions are considered to be irreversible since it is not possible to displace the equilibrium towards the formation of NCaSil by increasing the concentration of calcium 
or sodium silicate of solution [36]. Furthermore, contacting NCaSil with water will cause total loss of its initial features.

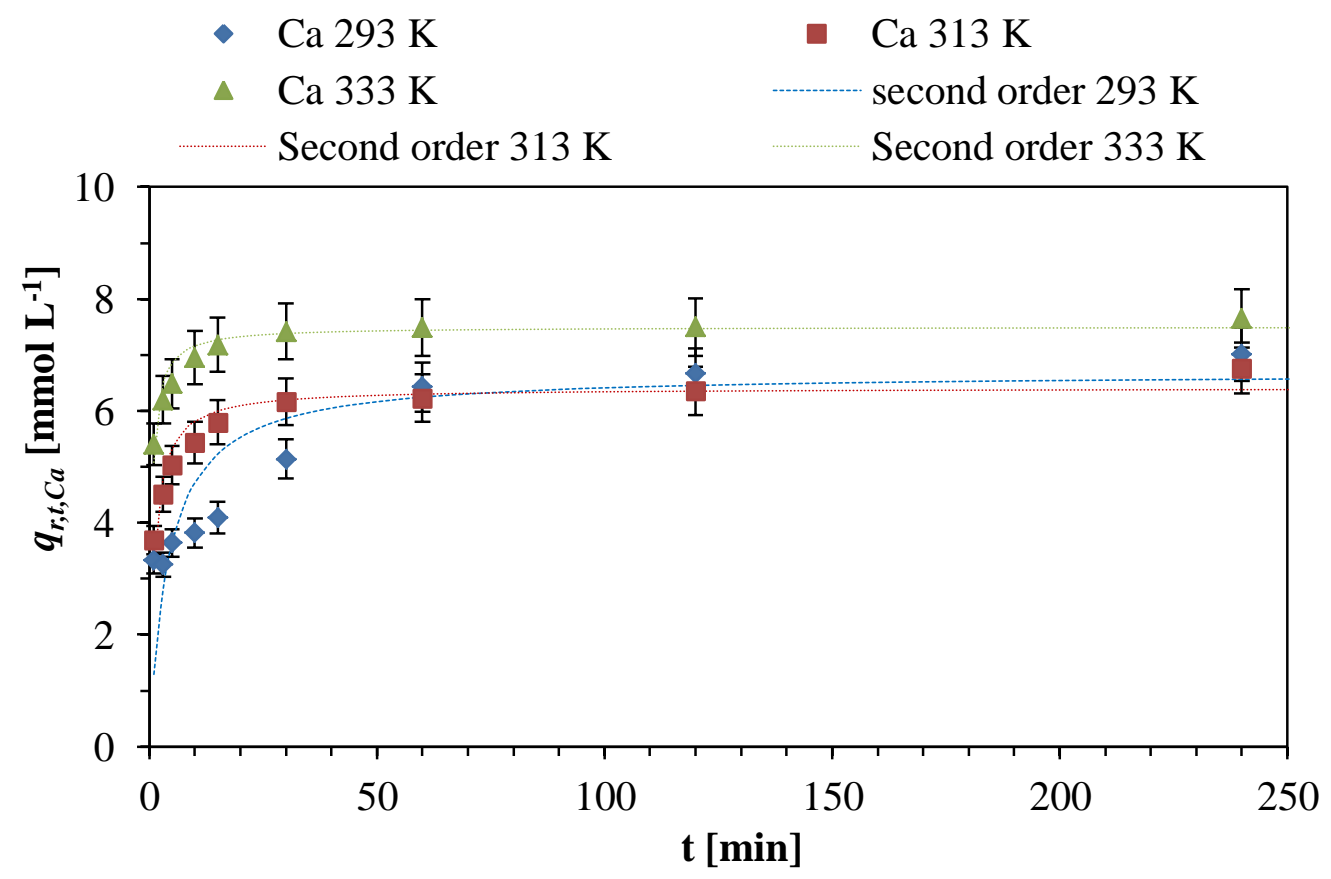

Figure 3.5: kinetic plot and pseudo-second order model fit for calcium release at 293, 313 and $333 \mathrm{~K}$. Experimental conditions: adsorbent dosage $1 \mathrm{~g} \mathrm{~L}^{-1}$; $\left[\mathrm{Cu}^{2+}\right]_{\text {initial }} 15.7 \mathrm{mmol} \mathrm{L}^{-1}$.

The total amount of $\mathrm{Ca}^{2+}$ ions released and the rate at which this species were transported into solution increased with temperature. Taking into consideration that the molar mass of NCaSil is approximately $138 \mathrm{~g} \mathrm{~mol}^{-1}$ (refer to Table 1.3), $1 \mathrm{~g}$ of NCaSil is equivalent to $7.3 \mathrm{mmol}$ of NCaSil. This value is close to that reported by McFarlane [36] where calcium is found in the range of $0.05-1 \mathrm{~mol}$ of Ca per mol of NCaSil.

The release of calcium ranged between 6.8 and $7.85 \mathrm{mmol} \mathrm{L}^{-1}$ suggesting all calcium was leached out when it is compared to the empirical formula. There is at most positive difference of $0.55 \mathrm{mmol} \mathrm{g}^{-1}(\approx 7 \%)$ of the total amount. Considering that the error of the method is $6.8 \%$ with a $95 \%$ confidence interval this difference lies within the error.

Kinetic parameters and the coefficient of determination for the second order equation fits are presented in Table 3.3.

Table 3.3: Pseudo-second order kinetic parameters for the release of calcium to the solution at 293, 313 and $333 \mathrm{~K}$ calculate from the data presented in Figure 3.5.

\begin{tabular}{|c|c|c|c|c|}
\hline $\begin{array}{c}\mathbf{T} \\
{[\mathbf{K}]}\end{array}$ & $\begin{array}{c}k \\
{\left[\mathrm{~L} \mathrm{mmol} \mathrm{mmin}^{-1}\right]}\end{array}$ & $\begin{array}{c}q_{r, e, C a} \\
{\left[\mathbf{m m o l ~ L}^{-1}\right]}\end{array}$ & $\begin{array}{c}k\left(q_{r, e, C a}\right)^{2} \\
{\left[\mathrm{mmol} \mathrm{L}^{-1} \min ^{-1}\right]}\end{array}$ & $r^{2}$ \\
\hline 293 & 0.03591 & 6.67884 & 1.60183 & 0.64274 \\
\hline 313 & 0.15161 & 6.40666 & 6.22288 & 0.88636 \\
\hline 333 & 0.28015 & 7.50005 & 15.7586 & 0.89071 \\
\hline
\end{tabular}


Calcium leaching exhibits a low correlation to a pseudo-first order model with $r^{2}$ values $<0.5441$ (not shown), while the pseudo-second order equation offers the best fit at all studied temperatures. Nevertheless, the $r^{2}$ value increases as the temperature increases for both orders. This may be an indication that the mechanism is changing with temperature. But to simplify the system in all studied temperatures the reaction will be considered as pseudo-second order. However, it is difficult to represent this pseudo-second order in a simple equation since in all reaction stated so far the release of calcium should proceed as a first order kinetic process when contacted with water. The inclusion of $\mathrm{Cu}^{2+}$ in the reaction may offer the chance to express calcium leach out as an irreversible reaction second order reaction as $\mathrm{A}+\mathrm{B} \rightarrow \mathrm{C}+\mathrm{D}$. In order to do this NCaSil should be considered as a reagent in the same phase rather than a solid (homogeneous rather than heterogeneous reaction). This could be true if the reaction is mediated by short oligomer chains in solution. Consequently the reaction could be rewritten as follows.

$$
\mathrm{CaSiO}_{2.53}(\mathrm{OH})_{0.25}+x \mathrm{Cu}^{2+} \longrightarrow y \mathrm{Ca}^{2+}+\mathrm{Cu}_{x}(\mathrm{OH})_{2 x} \mathrm{Ca}_{1-y} \mathrm{SiO}_{2.53}(\mathrm{OH})_{0.25-2 x} \text { (eq. 3.12) }
$$

But there is not enough empirical evidence to support that oligomers are involved in the uptake.

Comparing these results to those obtained for copper at similar temperatures, it is observed that at low temperatures $(\mathrm{T}<317 \mathrm{~K})$ calcium is leached faster than the rate at which copper ions are adsorbed. Nevertheless, at higher temperatures around 333K the kinetic rate constants and initial rates for the release of calcium and copper uptake are approximately of the same order of magnitude. Thus, the uptake of $\mathrm{Cu}^{2+}$ is affected to a greater extent by the temperature. In addition, the kinetic rate constant and the initial rate of $\mathrm{Ca}^{2+}$ leaching increases one order of magnitude with a $40 \mathrm{~K}$ increase in temperature. From the kinetic rate constants the activation energy for calcium leaching was calculated to be $41.9 \mathrm{~kJ} \mathrm{~mol}^{-1} \cdot \mathrm{Cu}^{2+}$ uptake had an activation energy of $54 \mathrm{~kJ} \mathrm{~mol}^{-1}$, therefore it is affected in a greater extent by changes in the temperature compared to calcium. In fact, this explains the reason why at higher temperatures copper uptake and calcium leaching have rate constants of similar magnitude.

If calcium ions are released faster than the amount of copper ions being removed from solution a negative charge on the surface should be observed. Klimsa [45] studied the variation of zeta potential over time for this reaction at $293 \mathrm{~K}$ using a copper sulfate solution showing consistent results to what it was expected for calcium leaching. The 
initial value of zeta potential was $-30 \mathrm{mV}$ and then moved towards more positive values near $-15 \mathrm{mV}$ remaining constant for the rest of the experiment. Zeta potential increased over time mainly due to the precipitation of copper on the surface, hence exhibiting another proof that crystal formation occurs on the surface rather than in solution. Additionally, the release of calcium might aid the adsorption of copper by increasing the negative charge on the surface, increasing the migration of copper ions onto the surface as shown in Klimsa's study [45]. In fact, this electrical potential gradient should aid mass transfer though the boundary layer.

\subsubsection{Kinetic study on monomeric silica release during the uptake of $\mathrm{Cu}^{2+}$.}

Figure 3.6 shows the release of $\mathrm{Si}(\mathrm{OH})_{4}$ (expressed as $\mathrm{mmol}$ of $\mathrm{SiO}_{2}$ per $\mathrm{L}$ of solution) when $1 \mathrm{~g}$ of NCaSil is contacted with a $15.7 \mathrm{mmol}$ of $\mathrm{Cu}^{2+}$ per L solution, the standard arrangement in this thesis.

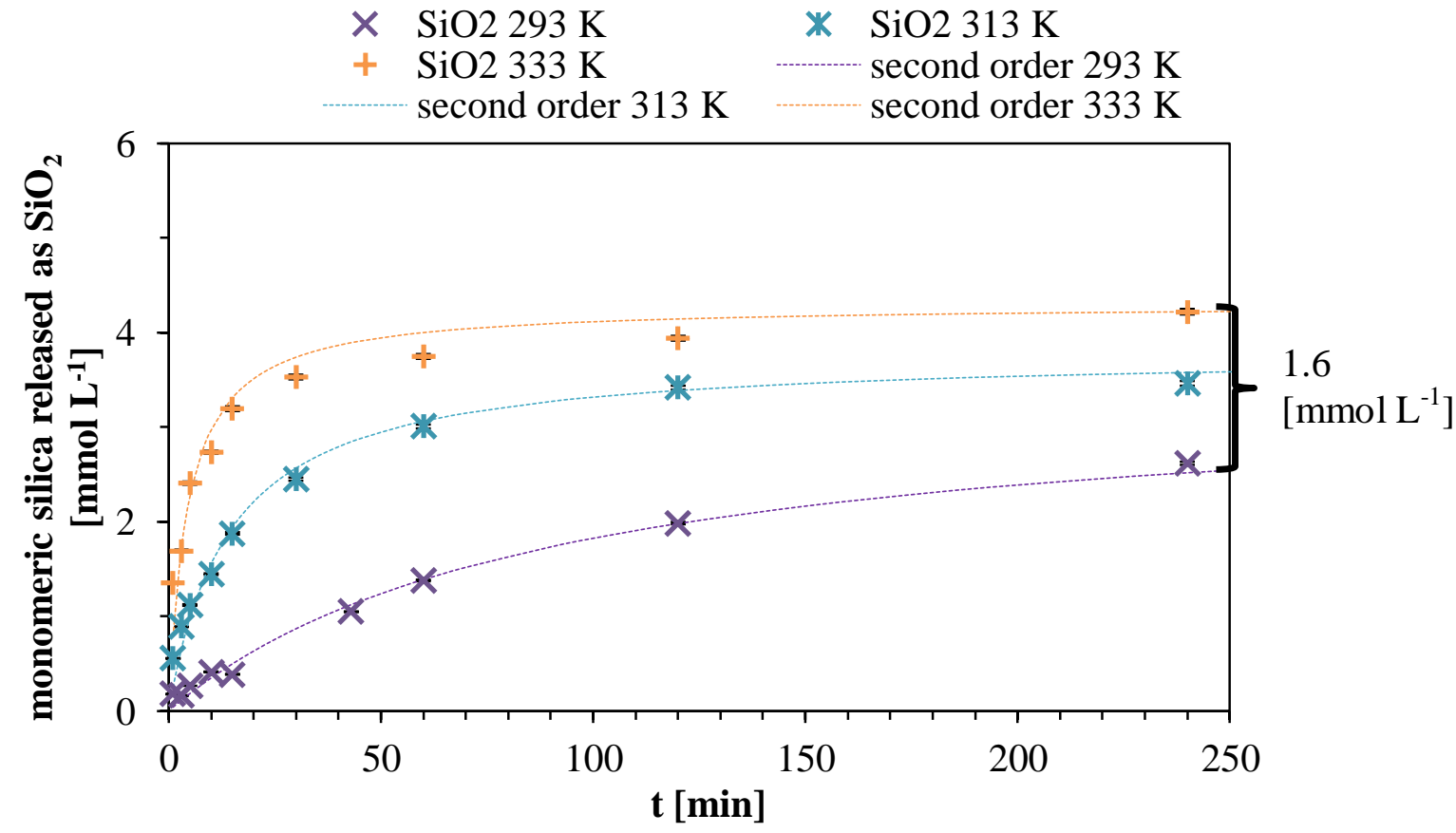

Figure 3.6: Kinetic plot and pseudo second order model fit for the release of monomeric silica from NCaSil at 293, 313 and $333 \mathrm{~K}$. Experimental conditions: adsorbent dosage 1 g $\mathrm{L}^{-1}$; $\left[\mathrm{Cu}^{2+}\right]_{\text {initial }} 15.7 \mathrm{mmol} \mathrm{L}^{-1}$.

The release of monomeric silica reached equilibrium after 240 min when the experiment was carried out at $293 \mathrm{~K}$, whereas at 313 and $333 \mathrm{~K}$ the equilibrium was reached approximately after 120 and $60 \mathrm{~min}$ respectively. In other words, every $20 \mathrm{~K}$ increment in the temperature the time necessary to reach equilibrium was reduced by half. The amount of monomeric silica released at the equilibrium increased approximately $1.6 \mathrm{mmol} \mathrm{L}^{-1}$ after a temperature increase of $40 \mathrm{~K}$ compared to the $0.9 \mathrm{mmol} \mathrm{L}^{-1}$ for $\mathrm{Ca}^{2+}$ release in the same range of temperature. Hence, twice as much monomeric silica 
than calcium is released with a $60 \mathrm{~K}$ increment on the temperature indicating that monomeric silica release is more temperature dependant than $\mathrm{Ca}^{2+}$ leaching. Release of $\mathrm{Ca}^{2+}$ ions may be dependent on other variables such as $\mathrm{pH}$.

Table 3.4: Pseudo-second order kinetic parameters for the release of monomeric silica into solution at 293, 313 and $333 \mathrm{~K}$, calculated from experimental data shown in Figure 3.6.

\begin{tabular}{|c|c|c|c|c|}
\hline $\begin{array}{c}\mathbf{T} \\
{[\mathbf{K}]}\end{array}$ & $\begin{array}{c}k \\
{\left[\mathrm{~L} \mathrm{mmol}^{-1} \mathrm{~min}^{-1}\right]}\end{array}$ & $\begin{array}{c}q_{r, e, S i O 2} \\
{\left[\mathrm{mmol} \mathrm{L}^{-1}\right]}\end{array}$ & $\begin{array}{c}k\left(q_{r, e, S i O 2}\right)^{2} \\
{\left[\mathrm{mmol} \mathrm{L}^{-1} \min ^{-1}\right]}\end{array}$ & $r^{2}$ \\
\hline 293 & 0.00324 & 3.453 & 0.039 & 0.99351 \\
\hline 313 & 0.01846 & 3.789 & 0.265 & 0.97866 \\
\hline 333 & 0.05185 & 4.297 & 0.957 & 0.89962 \\
\hline
\end{tabular}

The experimental data fitted to a first order equation exhibited a low correlation with $r^{2}<0.8$ (not shown) while the second order model had a better fit with $r^{2}>0.8996$ for all studied temperatures. Nevertheless, the coefficient of determination decreases as the temperature increases; this may suggest a change in the release mechanism, such as a release of different oligomeric silica species.

It is difficult to express a second order reaction mechanism for the release of monomeric silica while $\mathrm{Cu}^{2+}$ is adsorbed due to the large amount of parallel and chain reactions occurring with similar rate constant magnitudes.

Certainly a significant portion of the initial amount of NCaSil is released into solution and may be explained as a hydrolysis of the material which becomes promoted when heat is applied. Furthermore, contacting NCaSil with a solution with a low pH value breaks Si-O-Si and Si-O-Ca bonds releasing its constituents into solution.

Taking in consideration that in the empirical formula of NCaSil there is $1 \mathrm{~mol}$ of $\mathrm{Si}$ in every mole of NCaSil, it is possible to state that, $47-59 \%$ of the initial amount of $\mathrm{Si}$ in NCaSil was released into solution and is present as monomeric silica in solution at equilibrium.

Iler [67] measured the solubility for amorphous silica at different temperatures and $\mathrm{pH}$ values in solution which are shown in Figure 3.7. It is possible to extrapolate and approximate solubility values of 3.3, 4.0 and $5.0 \mathrm{mmol} \mathrm{L}^{-1}$ for 293,313 and $333 \mathrm{~K}$, at a $\mathrm{pH}$ value of 5 , respectively. This is consistent with the concentration found at the equilibrium in Figure 3.6 and Table 3.4. 


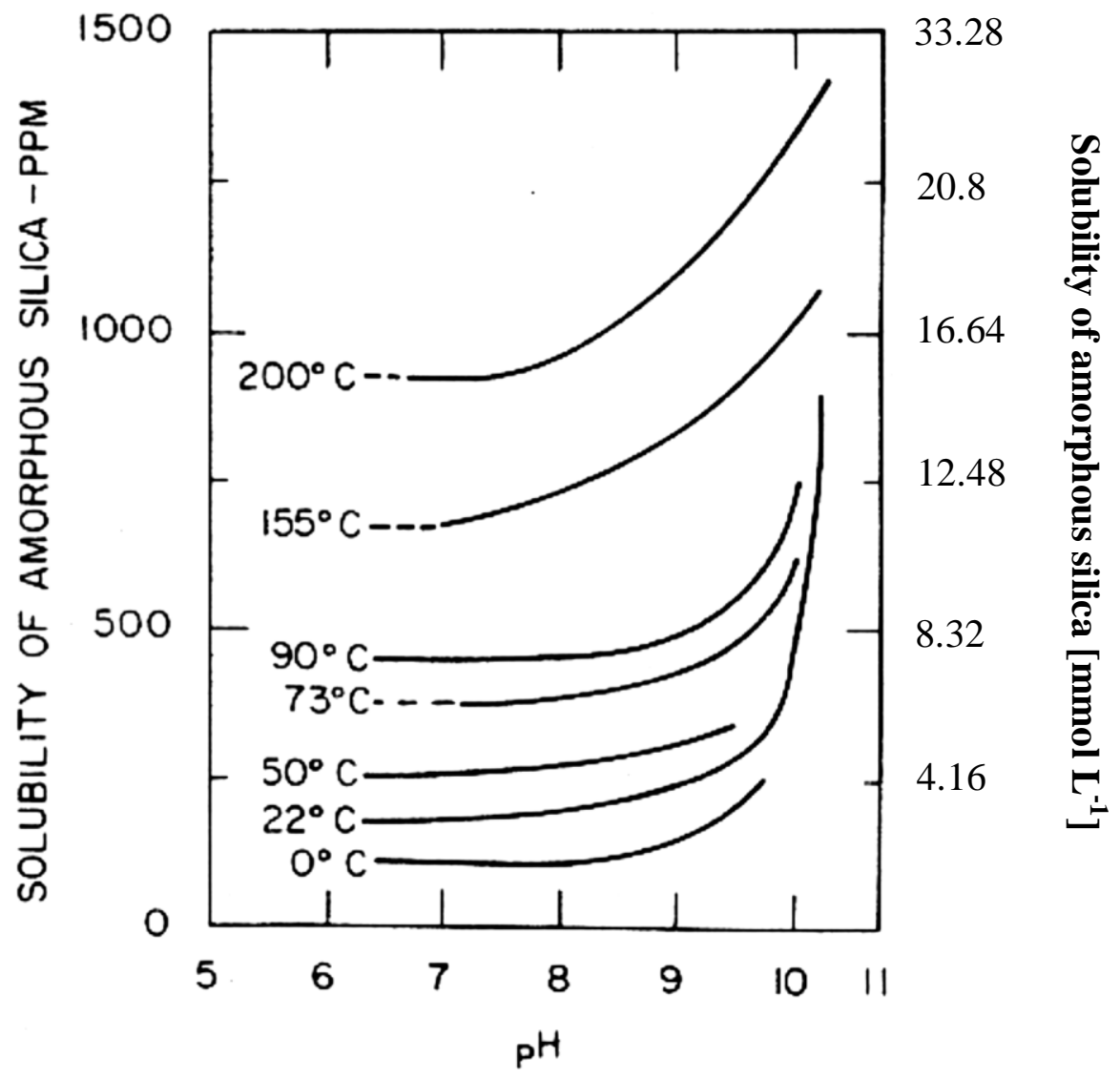

Figure 3.7: Solubility of amorphous silica at different temperatures over a pH range taken from reference [67].

Therefore the hydrolysis proceeds to a full extent until it reaches the maximum solubility of monomeric silica at a given temperature. Hence, the extent of the release of monomeric silica from NCaSil into solution is governed mainly by its solubility which is $\mathrm{pH}$ and temperature dependant. 


\subsubsection{Kinetic study of $\mathrm{OH}^{-}$release from NCaSil}

Cairns [37] reported that hydroxyl ions were released into solution over time as NCaSil was submerged in water. Nevertheless, the relation between hydroxyl ions released and the initial $\mathrm{Cu}^{2+}$ concentration has not yet been evaluated. Doing so may be an important parameter to understand the adsorption of copper since up to this point empirical evidence from previous studies [37,43] and this one suggests the formation of amorphous copper hydroxide as a first step in the reaction.

The variation in the $\mathrm{pH}$ of the solution was followed over time using different initial concentrations of $\mathrm{Cu}^{2+}$ in solution at three different temperatures $(293,303,313 \mathrm{~K})$. Results for the lowest studied temperature (293 K) are shown in Figure 3.8.

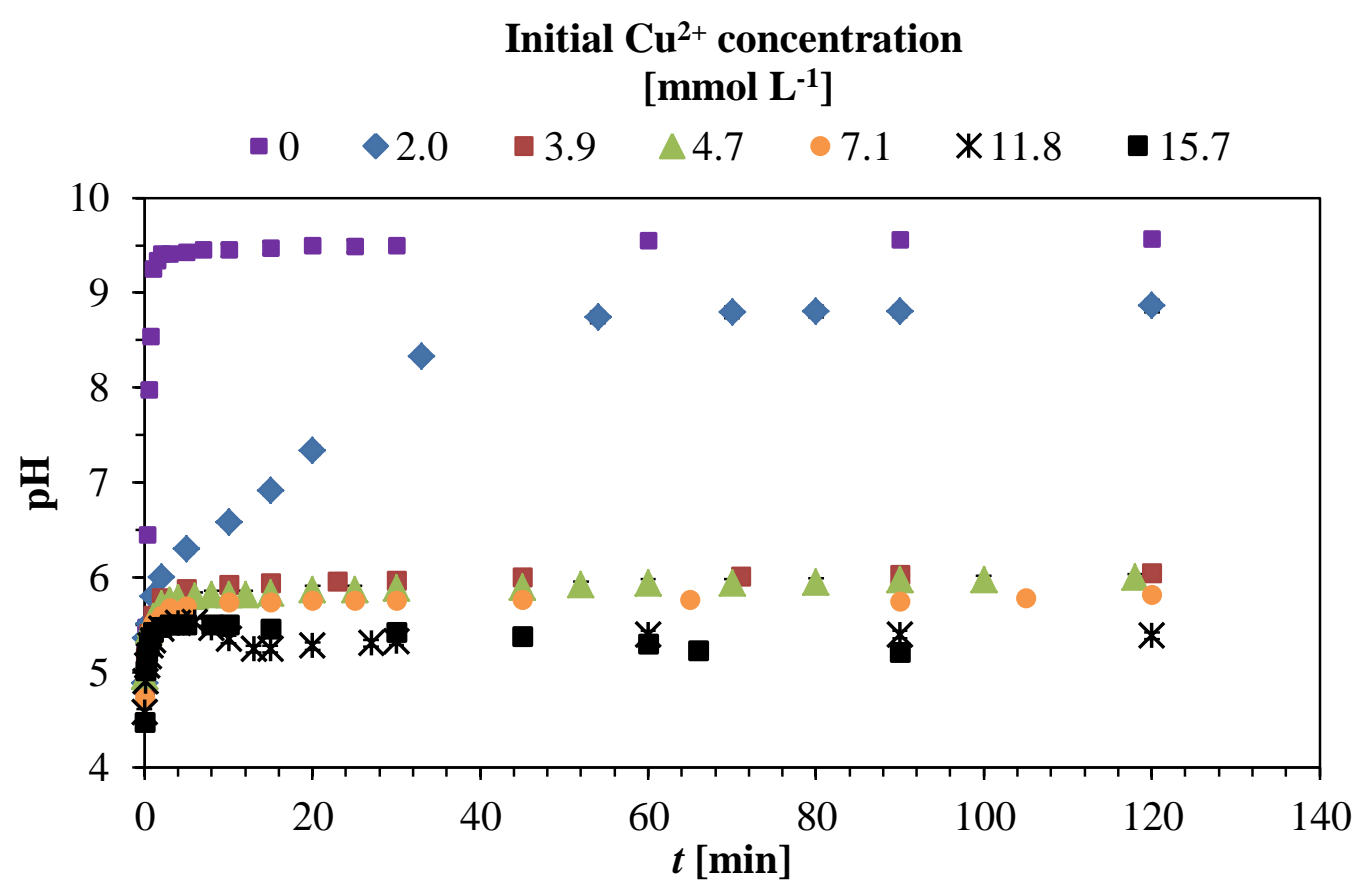

Figure 3.8: $\mathrm{pH}$ variation over time at $293 \mathrm{~K}$ for different initial concentration of $\mathrm{Cu}^{2+}$. Experimental conditions: adsorbent dosage $1 \mathrm{~g} \mathrm{~L}^{-1}$.

Without the presence of copper, $\mathrm{NCaSil}$ leaches $\mathrm{OH}^{-}$into solution increasing the $\mathrm{pH}$ to a value near 9.5. Hydroxyl leaching reaches equilibrium in the first minutes of the reaction and remains unvaried over time under these conditions. This result at $293 \mathrm{~K}$ is homologous to the one reported by Cairns [37] at the same temperature for $\mathrm{pH}$ variation. Hence, experiments are equivalent and reproducible.

If NCaSil is contacted with a $2.0 \mathrm{mmol} \mathrm{L}^{-1}$ solution of $\mathrm{Cu}^{2+}$ the variation of $\mathrm{pH}$ exhibits a fast rise in the first minute of the reaction and then slows down with a steady growth until it reaches equilibrium at $\mathrm{t} \approx 60 \mathrm{~min}$. This behaviour might be due to the presence of $\mathrm{HSO}_{4}{ }^{-}$and $\left[\mathrm{Cu}\left(\mathrm{H}_{2} \mathrm{O}\right)_{6}\right]^{2+},[\mathrm{Cu}(\mathrm{OH})]^{+}$and $\mathrm{Cu}(\mathrm{OH})_{2}$ could be buffering the $\mathrm{pH}$. Also the possibility of the chain scission of NCaSil structure could generate a buffer-like 
effect in the solution. Hence the process is complex due to the different $\mathrm{pK}_{\mathrm{a}}$ values of each compound.

At medium range initial concentrations between 2.0 and $7.1 \mathrm{mmol} \mathrm{L}^{-1}$ the $\mathrm{pH}$ increases rapidly to a value near 5.8 and stays constant during the first four hours. At a concentration of $15.7 \mathrm{mmol} \mathrm{L}^{-1}$ the $\mathrm{pH}$ increases up to a value of 5.6 at $10 \mathrm{~min}$ and drops towards a value of 5. This result is consistent to that Spark et al. [44] observed during the formation of copper nitrate hydroxide and copper chloride hydroxide minerals in solution. The authors stated this $\mathrm{pH}$ variation could be explained by the formation of $\mathrm{Cu}(\mathrm{OH})_{2}$ as an intermediate towards the formation of these minerals in solution. Hence, this could be an indication that $\mathrm{Cu}^{2+}$ forms amorphous copper hydroxide on the surface.

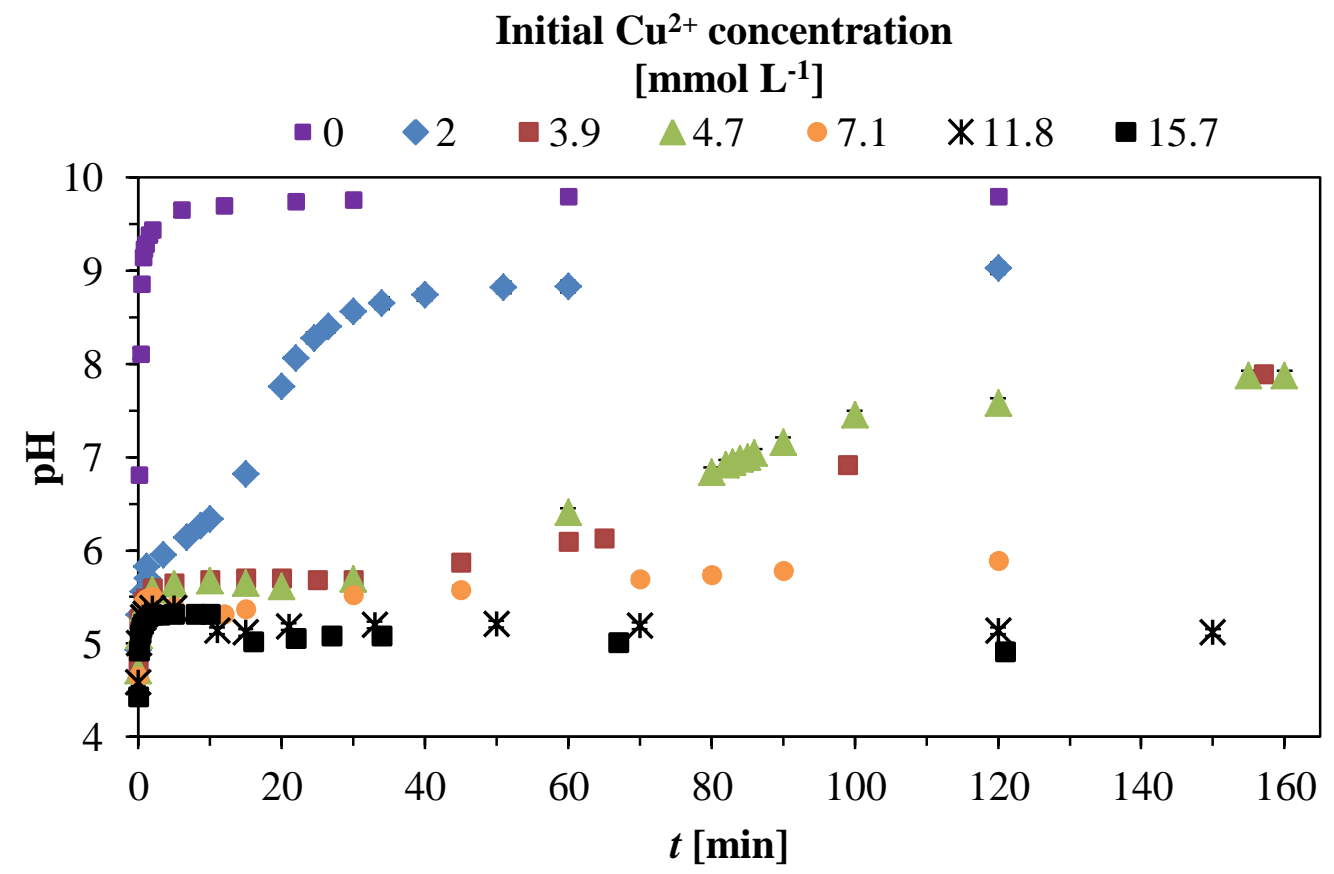

Figure 3.9: $\mathrm{pH}$ variation over time at $303 \mathrm{~K}$ with different initial concentration of $\mathrm{Cu}^{2+}$. Experimental conditions: adsorbent dosage $1 \mathrm{~g} \mathrm{~L}^{-1}$.

If the temperature is raised by $10 \mathrm{~K}$, hydrolysis of the $\mathrm{NCaSil}$ is faster and the $\mathrm{pH}$ reaches equilibrium values at shorter times as shown in Figure 3.9. It is clear that temperature plays a role on how quickly $\mathrm{Si}(\mathrm{OH})_{4}$ is released into solution and copper ions are adsorbed.

If $\mathrm{NCaSil}$ is contacted with a $2.0 \mathrm{mmol} \mathrm{L}^{-1}$ solution of $\mathrm{Cu}^{2+}$ the variation of $\mathrm{pH}$ exhibits a fast increase in the first minute of the reaction and then the rate slows down with a steady growth until it reaches equilibrium at $\mathrm{t} \approx 40 \mathrm{~min}, 20$ minutes faster than at $293 \mathrm{~K}$. At medium range initial concentrations between 2.0 and $7.1 \mathrm{mmol} \mathrm{L}^{-1}$ the $\mathrm{pH}$ increases rapidly to a value near $5.5(0.3 \mathrm{pH}$ unit less than $293 \mathrm{~K})$ and exhibits a sigmoidal growth 
for the rest of the experiment. This was not observed at $293 \mathrm{~K}$ as curves remained constant during 4 hours. At a concentration of $15.7 \mathrm{mmol} \mathrm{L}^{-1}$ the $\mathrm{pH}$ increases up to a value of 5.6 at $10 \mathrm{~min}$ and drops towards a value of 5 similar to the behaviour observed at $293 \mathrm{~K}$.

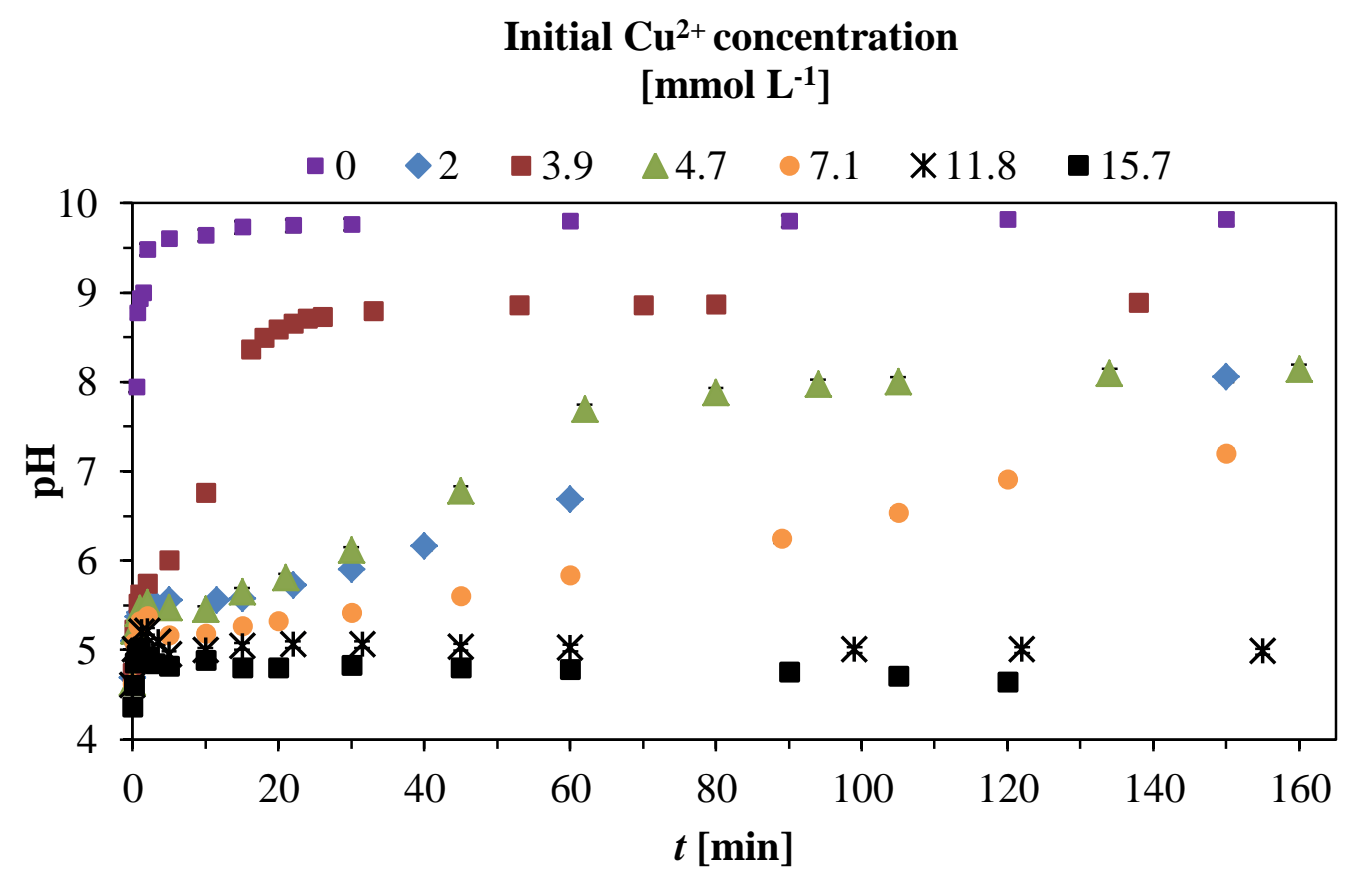

Figure 3.10: $\mathrm{pH}$ variation over time at $313 \mathrm{~K}$ with different initial concentration of $\mathrm{Cu}^{2+}$. Experimental condition: adsorbent dosage $1 \mathrm{~g} \mathrm{~L}^{-1}$.

At $313 \mathrm{~K}$ the effect of temperature on the variation of the $\mathrm{pH}$ in solution becomes even more pronounced, as shown in Figure 3.10. Curves are spread and trends may easily be spotted.

If NCaSil is contacted with a $2.0 \mathrm{mmol} \mathrm{L}^{-1}$ solution of $\mathrm{Cu}^{2+}$ the variation of $\mathrm{pH}$ exhibits a fast increase in the first minute of the reaction and then the rate slows down with a steady growth until equilibrium is reached at $\mathrm{t} \approx 20 \mathrm{~min}$, being 40 minutes sooner than at $293 \mathrm{~K}$. At medium range initial concentrations between 2.0 and $7.1 \mathrm{mmol} \mathrm{L}^{-1}$ the $\mathrm{pH}$ increases rapidly to a value near $5-5.5$ and exhibits a sigmoidal growth for the rest of the experiment. This was not observed at $293 \mathrm{~K}$ as curves remained constant. At a concentration of $15.7 \mathrm{mmol} \mathrm{L}^{-1}$ the $\mathrm{pH}$ increases up to a value of 5.6 at $3 \mathrm{~min}$ and drops rapidly to a value of 4.6. This is consistent to what is mentioned in the literature as the stable $\mathrm{pH}$ at which brochantite $\left(\mathrm{Cu}_{4}(\mathrm{OH})_{6} \mathrm{SO}_{4}\right)$ forms readily [44]. 
Table 3.5: pH values at the equilibrium.

\begin{tabular}{|c|c|c|c|}
\hline $\begin{array}{c}\mathbf{T} \\
{[\mathbf{K}]}\end{array}$ & $\begin{array}{c}\mathrm{Cu}^{2+} \text { initial conc. } \\
{\left[\mathrm{mmol} \mathrm{L}^{-1}\right]}\end{array}$ & $\begin{array}{c}\mathrm{pH} \\
\text { at } t=0\end{array}$ & $\begin{array}{c}\mathrm{pH} t=24 \mathrm{~h}, \\
\text { at equilibrium }\end{array}$ \\
\hline \multirow{8}{*}{293} & 0 & 5.22 & 9.62 \\
\hline & 2.0 & 4.89 & 8.79 \\
\hline & 3.9 & 4.82 & 8.46 \\
\hline & 4.7 & 4.65 & 8.17 \\
\hline & 6.3 & 4.60 & 7.25 \\
\hline & 7.1 & 4.49 & 6.43 \\
\hline & 11.8 & 4.25 & 5.31 \\
\hline & 15.7 & 4.48 & 4.83 \\
\hline \multirow{8}{*}{303} & 0 & 5.23 & 9.80 \\
\hline & 2.0 & 4.93 & 9.03 \\
\hline & 3.9 & 4.82 & 8.60 \\
\hline & 4.7 & 4.71 & 8.40 \\
\hline & 6.3 & 4.75 & 7.40 \\
\hline & 7.1 & 4.65 & 6.32 \\
\hline & 11.8 & 4.58 & 5.24 \\
\hline & 15.7 & 4.43 & 4.58 \\
\hline \multirow{8}{*}{313} & 0 & 5.20 & 9.84 \\
\hline & 2.0 & 4.74 & 8.92 \\
\hline & 3.9 & 4.69 & 8.72 \\
\hline & 4.7 & 4.63 & 8.32 \\
\hline & 6.3 & 4.66 & 8.14 \\
\hline & 7.1 & 4.62 & 8.00 \\
\hline & 11.8 & 4.60 & 5.05 \\
\hline & 15.7 & 4.36 & 4.40 \\
\hline
\end{tabular}

In Table 3.5 it may be observed that at initial concentrations of $\mathrm{Cu}^{2+}$ higher than $11.8 \mathrm{mmol} \mathrm{L}^{-1}$ the $\mathrm{pH}$ value tends to reach equilibrium close to its initial value, following the trend observed in the plots. These $\mathrm{pH}$ values at the equilibrium are important since they will directly influence on the amount of monomeric silica at the equilibrium as shown in Figure 3.7 and appendix A-3.

The $\mathrm{pH}$ tends to return to its initial values due to an excess of protons, which in this case is related to an excess of $\mathrm{Cu}^{2+}$ which is hydrolysed in solution as shown eq. 3.14 [68].

$\mathrm{M}^{2+}\left(\mathrm{H}_{2} \mathrm{O}\right)_{\mathrm{n}} \rightleftharpoons \mathrm{M}(\mathrm{H} 2 \mathrm{O})_{\mathrm{n}-1}(\mathrm{OH})^{+}+\mathrm{H}^{+}$

For copper, $\mathrm{M}=\mathrm{Cu}$ and $\mathrm{n}=6$. The equilibrium shown in eq. 3.14 has a $\mathrm{pK}$ value of 8 .

At lower initial concentrations of $\mathrm{Cu}^{2+}$ the concentration of protons is low and becomes neutralized by $\mathrm{OH}^{-}$present and/or released from $\mathrm{NCaSil}$. 


\subsubsection{Powder-XRD study on the kinetic of crystal growth of copper minerals on the surface of NCaSil at different temperatures}

The intensity of a reflection of a crystallographic plane in an XRD pattern is related to the atoms in the crystal structure. Those atoms located nearer to the plane will have a greater contribution compared to those located far from the plane inside a unit cell. This

relation is given by the structure factor, $F_{(h k l)}=\sum_{j=1}^{n} f_{j} e^{2 \pi i\left(h x_{j}+k y_{j}+l z_{j}\right)}$, where $f_{j}$ is the atomic scattering factor which is related to the electronic property of the atom and the remaining exponential term is related to the position of that atom. The square of the structure factor is proportional to the reflection intensity, $\left|F_{(h k l)}\right|^{2} \propto I_{(h k l)}$, which is obtained experimentally.

Once the intensity is obtained it is possible to calculate the peak area by computational fitting. Furthermore, the area underneath the peak is proportional to the concentration. Therefore, similar models to those used throughout sections 3.2.1 to 3.2.4 were employed to obtain kinetic rate constants which allowed a quantitative comparison between the growth rates of different crystallographic planes. This allowed studying the kinetics of nucleation of $\mathrm{Cu}^{2+}, \mathrm{OH}^{-}$and $\mathrm{SO}_{4}{ }^{2-}$ forming brochantite $\left(\mathrm{Cu}_{4}(\mathrm{OH})_{6} \mathrm{SO}_{4}\right)$, which in turn help to elucidate which of these ions are transferred to the surface faster. Additionally, experiments were carried out at different temperatures to see dependence on the crystal growth on this variable and to obtain the activation energy for the development of an individual plane. This information will be contrasted with that gathered regarding the concentration of $\mathrm{Cu}^{2+}$ on the surface in section 3.2.2.

Figure 3.11 shows the results obtained at $293 \mathrm{~K}$ using powder-XRD to analyse the solid portion of the filtrate collected at different time intervals. 


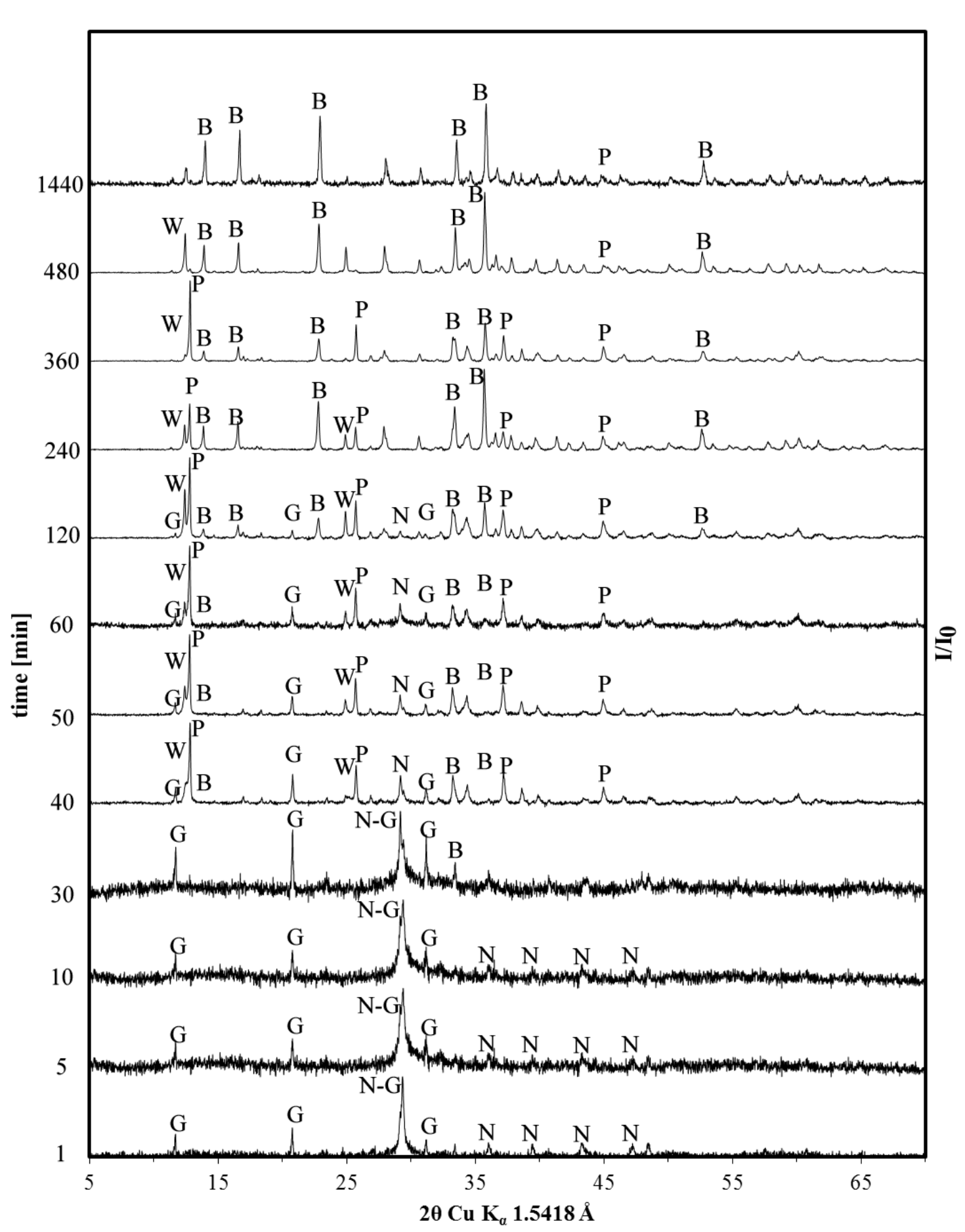

Figure 3.11: Kinetic study at $293 \mathrm{~K}$ of crystal growth on NCaSil using powder XRD. $\mathbf{G}=$ calcium sulfate dihydrate, $\mathbf{N}=$ NCaSil, $\mathrm{W}=$ wroewolfeite, $\mathbf{P}=$ posnjakite and $\mathrm{B}=$ brochantite. Experimental conditions: $\left[\mathrm{Cu}^{2+}\right]$ initial $15.7 \mathrm{mmol} \mathrm{L} \mathrm{L}^{-1}$; adsorbent dosage $1 \mathrm{~g} \mathrm{~L}^{-1}$; stirring speed $500 \mathrm{rpm}$.

The XRD patterns shown in Figure 3.11 presents the development of different crystal phases on the NCaSil. Five different compounds were identified in the samples for this temperature: calcium sulfate dehydrate $(\mathrm{G}), \mathrm{NCaSil}(\mathrm{N})$, wroewolfeite $(\mathrm{W})$, posnjakite (P) and brochantite (B). Wroewolfeite, posnjakite and brochantite are copper sulfate hydroxide minerals which are monoclinic and share the same chemical composition but differ in the amount of waters of crystallization. Their generic formula is: 
Where $n$ is equal to 2 in wroewolfeite, 1 in posnjakite and 0 in brochantite.

In the previous section a shift from blue to a turquoise colour during the uptake was mentioned. At $293 \mathrm{~K}$ the change occurred $20 \mathrm{~h}$ after starting the experiment, whereas at $333 \mathrm{~K}$ this occurred after 30 minutes. Analysing the powder-XRD pattern obtained for $293 \mathrm{~K}$ in Figure 3.11 one can relate this to the fact that brochantite becomes the predominant mineral phase at large values of $t$. This turquoise colour is consistent to the mixture of blue coloured crystals of wroewolfeite and posnjakite combined with those of brochantite, which are green, as shown in Figure 3.12. The formation of brochantite is consistent with the study done by Cairns [37] where salts of the form $\mathrm{Cu}_{2} \mathrm{X}(\mathrm{OH})_{3}$ were obtained, where $\mathrm{X}=\mathrm{NO}_{3}{ }^{-}$or $\mathrm{Cl}^{-}$.

Therefore a more general formula may be employed to describe the minerals formed over NCaSil;

$\mathrm{Cu}_{2 n} \mathrm{X}(\mathrm{OH})_{3 n}$

where $\mathrm{X}$ is an anion and $n$ is the charge of anion $\mathrm{X}$. All minerals that have been obtained so far may be described by this expression.

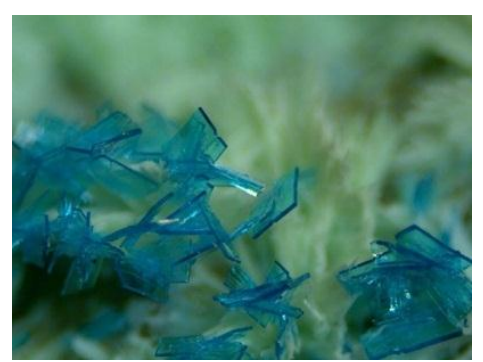

Wroewolfeite crystals, image taken from ref. [69]

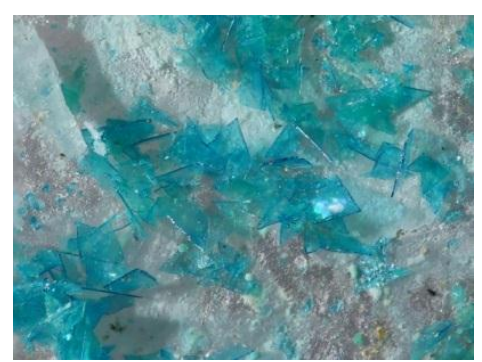

Posnjakite crystals, image taken from ref. [70]

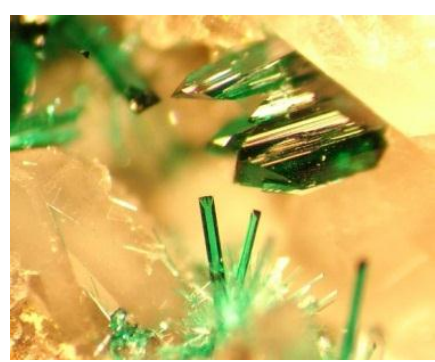

Brochantite crystals, image taken from ref. [71]

Figure 3.12: Examples taken from the literature for the three copper sulfate hydroxide minerals observed in this study.

In Figure 3.11, peaks corresponding to the development of gypsum are observed in the first minutes of the uptake (1-5 min). On the other hand, the intensity of the broad NCaSil peak described by McFarlane [36] near $\left.30^{\circ} 2 \theta(\mathrm{Cu} \mathrm{K})_{\alpha}\right)$ decreases, which in turn diminishes the signal to noise ratio. Between 15 and 30 minutes into the reaction NCaSil peaks have faded even more making the gypsum peaks look larger than they were at the beginning of the experiment. Between 30 and 40 minutes after commencing the reaction the simultaneous formation of wroewolfeite, posnjakite and brochantite is observed. Zamana and Usmanov [72] performed a theoretical thermodynamic study on 
the formation of this copper sulfate hydroxide hydrate species calculating $\Delta \mathrm{H}_{f}$ and $\Delta \mathrm{G}_{f}$ for different copper sulfate hydroxide minerals. They found that the energy requirements to form the different minerals starting from their ions in solution were as follows $\mathrm{W}<\mathrm{P}<\mathrm{B}$. This is in concordance with the results obtained at $293 \mathrm{~K}$ where the formation of $\mathrm{W}$ and $\mathrm{P}$ is favoured at short times.

Between 50 and 120 min into the reaction, the posnjakite reflection corresponding to the plane 001 at $12.7^{\circ} 2 \theta$ is considerably larger than the rest of the peaks. The development of this peak coincides with the uptake of copper reaching $\approx 80-100 \%$ of the loading capacity under these experimental conditions (refer to Figure 3.3). It is important to note that the wroewolfeite peak at $12.3^{\circ} 2 \theta$ is overlapped by the peak at $12.7^{\circ} 2 \theta$ but becomes more defined after 120 min into the reaction, suggesting wroewolfeite and/or posnjakite crystals become larger as the peaks become narrower. The calcium sulfate dihydrate peak at $11.6^{\circ} 2 \theta$ fades after $120 \mathrm{~min}$. This could be an indication that sulfate diffuses from the gypsum lattice into a nearby the copper sulfate hydroxide lattice but there is not enough evidence from the results to support this idea, other than the fact that calcium sulfate dihydrate disappears from the solid phase even when it has a $\mathrm{K}_{\mathrm{sp}}$ value of $2.5 \times 10^{-4}$.

From 240 to 1440 min the peak due to NCaSil is no longer observed. This is consistent with the collected samples which did not have NCaSil in the sample. Moreover, the main process occurring in this time frame is the transformation of wroewolfeite and posnjakite to brochantite. If enough time is allowed $(t \geq 1440)$ min the product is brochantite. Hence, this suggests that if the experiments are done at higher temperatures only small quantities of wroewolfeite and posnjakite should be formed while brochantite becomes the predominant phase. Taking into consideration that the chemical formulae of the substances differ in the number of crystallization water molecules the energy to transform wroewolfeite and posnjakite into brochantite will be strongly related to the amount of $\mathrm{H}_{2} \mathrm{O}$ molecules needed to be removed from the lattice. It is possible to relate the magnitude the activation energy of dehydration of copper sulfate trihydrate to those of wroewolfeite and posnajkite considering that all of them are monoclinic and disregarding the position of the water molecules inside the crystal structure. Cooper et al. [73] reported a value of $65.2 \mathrm{~kJ} \mathrm{~mol}^{-1}$ for the dehydration reaction expressed in eq. 3.17 .

$\mathrm{CuSO}_{4} \cdot 3 \mathrm{H}_{2} \mathrm{O} \underset{-2 \mathrm{H}_{2} \mathrm{O}}{\stackrel{\Delta}{\longrightarrow}} \mathrm{CuSO}_{4} \cdot \mathrm{H}_{2} \mathrm{O}$ 
The activation energy for one mole of water (half a mole of reaction) being displaced from the crystal lattice will be $32.6 \mathrm{~kJ} \mathrm{~mol}^{-1}$. This value will serve as comparison to those obtained later in this thesis.

With the information gathered in this study it is possible to write an expression for a stepwise reaction at $293 \mathrm{~K}$. Eq. 3.18 vaguely represents the undergoing chemical reactions, but gives a simple and clear idea of how the reaction proceeds towards the formation of brochantite on the surface. It is noteworthy that NCaSil particle disaggregation due to stirring is not included in this equation but must be considered in the overall process.

$$
\begin{aligned}
& \mathrm{Cu}_{(a q)}^{2+}+\mathrm{SO}_{4}^{2-}{ }_{(a q)}+\mathrm{NCaSil}_{(s)} \rightarrow \mathrm{Cu}(\mathrm{OH})_{2}-\mathrm{NCaSil}-\mathrm{CaSO}_{4(s)} \rightarrow \\
& \mathrm{Cu}_{4}(\mathrm{OH})_{6} \mathrm{SO}_{4} \cdot 2 \mathrm{H}_{2} \mathrm{O} \underset{-\mathrm{H}_{2} \mathrm{O}}{\longrightarrow} \mathrm{Cu}_{4}(\mathrm{OH})_{6} \mathrm{SO}_{4} \cdot \mathrm{H}_{2} \mathrm{O} \underset{-\mathrm{H}_{2} \mathrm{O}}{\longrightarrow} \mathrm{Cu}_{4}(\mathrm{OH})_{6} \mathrm{SO}_{4}
\end{aligned}
$$

Consequently, the first step of the reaction involves the disaggregation of the particles of NCaSil into asymmetric aggregates with an average of 20 micrometres in length exposing a large reactive surface. $\mathrm{Cu}^{2+}$ binds rapidly with the surface $\mathrm{OH}^{-}$groups and $\mathrm{SO}_{4}{ }^{2-}$ forms gypsum with $\mathrm{Ca}^{2+}$ available on the $\mathrm{NCaSil}$ structure. At $293 \mathrm{~K}$ this the first peak of brochantite develops after 30 minutes (refer to Figure 3.11). Nevertheless after 40 minutes of starting the experiment wroewolfeite and posnjakite become the predominant phase until $t=480 \mathrm{~min}$. Finally, if the amount of time is sufficient, brochantite forms as it is the thermodynamically stable compound. NCaSil is necessary in the reaction to form brochantite as it provides the $\mathrm{OH}^{-}$on the surface for the crystal to develop.

In the literature, this stepwise formation of minerals is referred to it as an Ostwald cascade, and has been described in the same order in the literature [74]. Nonetheless, so far it has not been able to obtain wroewolfeite at temperatures higher than $277 \mathrm{~K}$ starting from its constituents in solution [74].

Moreover, the synthesis of these crystals in solution is non-commutative meaning that the order in which we add the reagents will impact the resulting product. For example, the addition of $\mathrm{CuSO}_{4}$ to base initially gives a metastable precipitate of crystalline $\mathrm{Cu}(\mathrm{OH})_{2}$ (spertiniite), and adding base to a $\mathrm{CuSO}_{4}$ solution gives a metastable precipitate of posnjakite [74]. The latter is consistent to what is shown in this study where $\mathrm{NCaSil}$ was added into a $\mathrm{CuSO}_{4}$ solution.

In addition, the synthesis of these crystals could represent an industrial opportunity since they are the main compounds of the Bordeaux mixture use as fungicide in large 
scale agriculture [75]. The standard synthesis for Bordeaux mixture consists adding $1.8 \mathrm{~kg}$ of copper sulfate to $1.8 \mathrm{~kg}$ of hydrated lime in $188 \mathrm{~L}$ of water [76]. This translates into $3.8 \times 10^{-2} \mathrm{~mol} \mathrm{~L}^{-1}$ of $\mathrm{Cu}^{2+}$ against $1.57 \times 10^{-2} \mathrm{~mol} \mathrm{Cu}^{2+} \mathrm{L}^{-1}$ used in this study, hence twice as much $\mathrm{Cu}^{2+}$ is needed in the standard synthesis to obtain the product. X-ray analysis of a standard Bordeaux mixture in the literature [77] revealed that it consisted of devillite $\mathrm{Ca}\left[\mathrm{Cu}_{4}\left(\mathrm{SO}_{4}\right)_{2}(\mathrm{OH})_{6}\right] \cdot 2 \mathrm{H}_{2} \mathrm{O}$, posnjakite $\mathrm{Cu}_{4}(\mathrm{OH})_{6} \mathrm{SO}_{4} \cdot \mathrm{H}_{2} \mathrm{O}$, brochantite $\mathrm{Cu}_{4}(\mathrm{OH})_{6} \mathrm{SO}_{4}$, antlerite $\mathrm{Cu}_{3}\left(\mathrm{SO}_{4}\right)(\mathrm{OH})_{4}$, gypsum $\mathrm{CaSO}_{4} \cdot 2 \mathrm{H}_{2} \mathrm{O}$ and bassanite $\mathrm{CaSO}_{4} \cdot 0.5 \mathrm{H}_{2} \mathrm{O}$. In some cases synthesis of these compounds require a $\mathrm{Cu}^{2+}$ concentration of at least 1 order of magnitude larger compared to the amounts used in this study $[74,77]$.

Commercial Bordeaux mixtures usually form sticky deposits which plug the nozzle of spraying devices [77]. This is due to the presence of bassanite formed during the preparation of these minerals as a result of using lime as a reagent. Furthermore, patents on the synthesis and application of these crystals have been issued recently [76-79], hence there is still great interest in finding new ways of preparation of these copper sulfate hydroxide minerals.

From this study a bassanite free brochantite may be produced at room temperature from relatively low concentrations of $\mathrm{Cu}^{2+}$ using $\mathrm{NCaSil}$ as a substrate for crystal growth. If a mixture of different mineral phases is wanted it is possible to stop the reaction between 60 and 120 min obtaining a high yield of wroewolfeite, posnjakite and brochantite at room temperature as shown in Figure 3.11. From a scientific point of view this represents also an achievement as these minerals have not yet been isolated from experiments done in solution at room temperature [74]. Furthermore, the author believes that the results shown in this study might serve as an indication of how these minerals form in nature. 


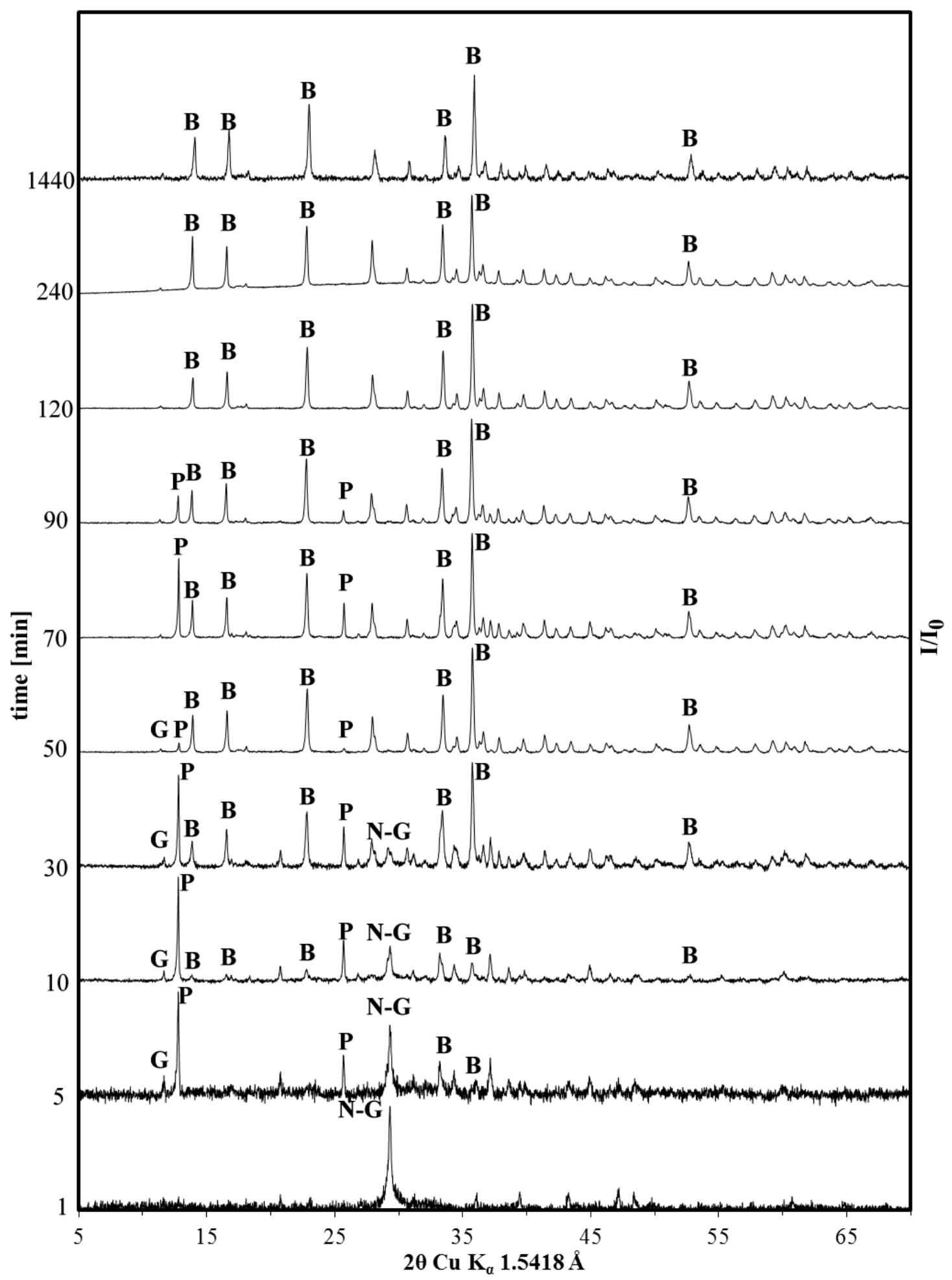

Figure 3.13: Kinetic study at $313 \mathrm{~K}$ of crystal growth on NCaSil using powder XRD. $\mathrm{G}=$ calcium sulfate dihydrate, $\mathbf{N}=$ NCaSil, $\mathbf{P}=$ posnjakite and $\mathbf{B}=$ brochantite. Experimental conditions: $\left[\mathrm{Cu}^{2+}\right]$ initial $15.7 \mathrm{mmol} \mathrm{L}^{-1}$; adsorbent dosage $1 \mathrm{~g} \mathrm{~L}^{-1}$; stirring speed $500 \mathrm{rpm}$.

In order to determine to what extent the temperature increases the rate of formation of brochantite in the reaction, the experiment was carried out at $313 \mathrm{~K}(+20 \mathrm{~K}$ difference from Figure 3.11). In Figure 3.13 the experimental results are depicted. During the first five minutes the rapid formation of posnjakite and brochantite may be observed without presence of wroewolfeite. This result is in agreement with the ease of formation due to 
energy requirements as discussed earlier in this section. Brochantite rapidly becomes the predominant crystal structure after $30 \mathrm{~min}$. NCaSil and calcium sulfate dihydrate peaks fade rapidly in time compared to those shown in Figure 3.11. In fact after 50 minutes only a small peak of calcium sulfate dihydrate may be observed, but in order to simplify the system it may be considered to have completely disappeared. The author believes that the fading of gypsum peak may be related to the surface diffusion of sulfate from gypsum to wroewolfeite, posnjakite or brochantite lattices since as the rate of crystal formation increases, the rate of disappearance of calcium sulfate dihydrate peaks also increases. Still this is statement is not properly supported and should be considered only as a hint of what is occurring on the surface. Between 50 and 70 minutes after started the reaction posnjakite develops faster than brochantite, but starts to fade after 90 minutes being undetectable at $120 \mathrm{~min}$. Brochantite peaks remain largely unchanged from 120 until 1440 minutes.

In general one could modify eq. 3.18 to the following expression:

$$
\begin{aligned}
& \mathrm{Cu}_{(a q)}^{2+}+\mathrm{SO}_{4}^{2-}{ }_{(a q)}+\mathrm{NCaSil}_{(s)} \rightarrow \mathrm{Cu}(\mathrm{OH})_{2}-\mathrm{NCaSil}_{-} \mathrm{CaSO}_{4} \rightarrow \\
& \mathrm{Cu}_{4}(\mathrm{OH})_{6} \mathrm{SO}_{4} \cdot \mathrm{H}_{2} \mathrm{O} \underset{-\mathrm{H}_{2} \mathrm{O}}{\longrightarrow} \mathrm{Cu}_{4}(\mathrm{OH})_{6} \mathrm{SO}_{4}
\end{aligned}
$$

Where wroewolfeite is not formed and the reaction proceeds directly to form posnjakite, which then dehydrates to form brochantite. 


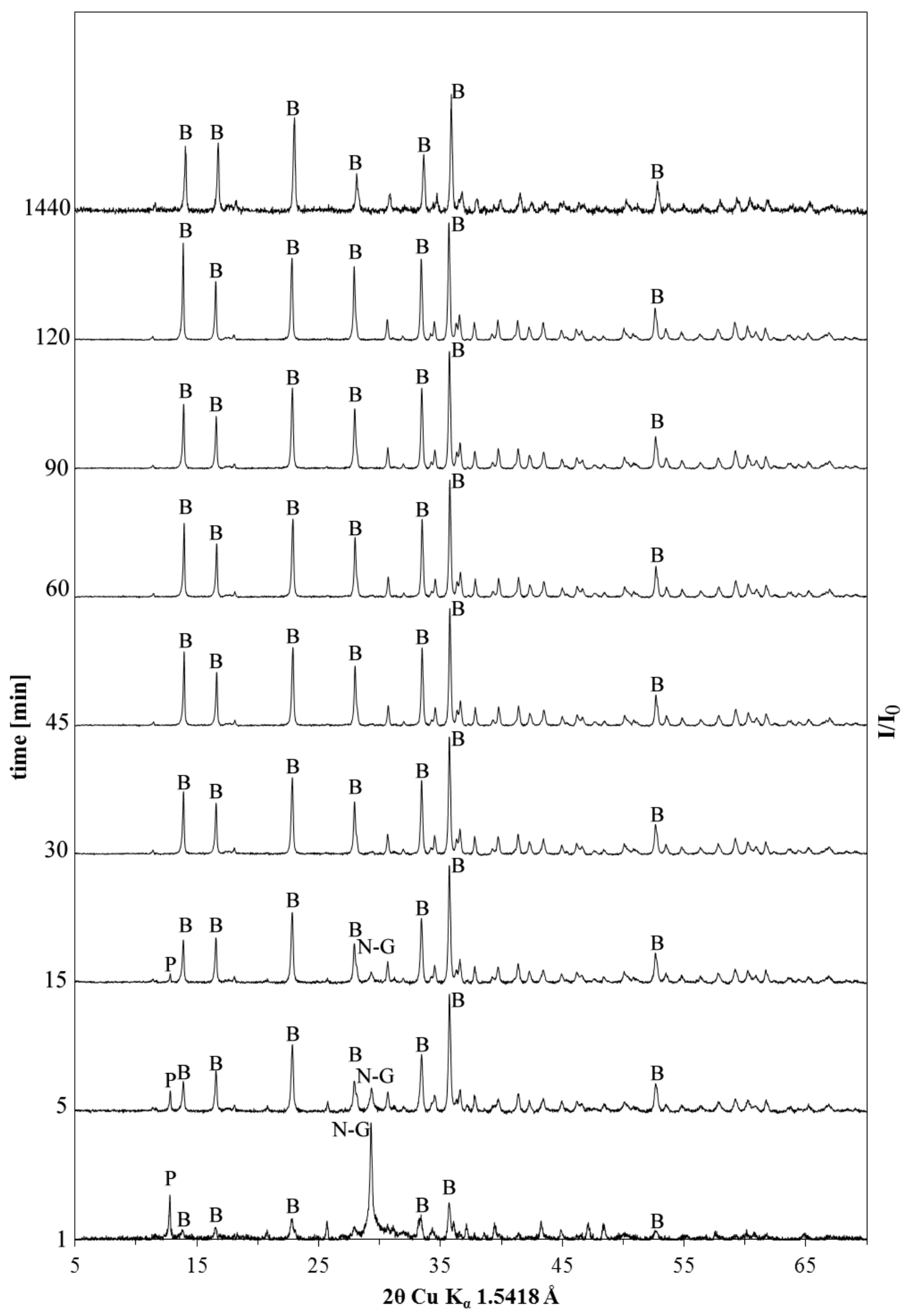

Figure 3.14: Kinetic study at $333 \mathrm{~K}$ of crystal growth on NCaSil using powder XRD. $\mathrm{G}=$ calcium sulfate dihydrate, $\mathbf{N}=$ NCaSil, $\mathbf{P}=$ posnjakite and $\mathbf{B}=$ brochantite. Experimental conditions: $\left[\mathrm{Cu}^{2+}\right]$ initial $15.7 \mathrm{mmol} \mathrm{L}{ }^{-1}$; adsorbent dosage $1 \mathrm{~g} \mathrm{~L}^{-1}$; stirring speed $500 \mathrm{rpm}$.

The experiment was repeated at $333 \mathrm{~K}$ and the same four phases, NCaSil, calcium sulfate dihydrate, posnjakite and brochantite may be identified in Figure 3.14. Raising 
the temperature by $20 \mathrm{~K}$ increases the rate of crystal growth of brochantite, resulting in posnjakite being rapidly dehydrated during the first 15 minutes to form brochantite. After 30 minutes the powder-XRD patterns remain almost constant. Regarding these results it is possible to make the assumption there is enough energy in the system to form brochantite without the need forming posnjakite first. So the formation of brochantite at temperatures higher than $333 \mathrm{~K}$ may be described by the following expression.

$$
\begin{aligned}
& \mathrm{Cu}_{(a q)}^{2+}+\mathrm{SO}_{4}^{2-}{ }_{(a q)}+\mathrm{NCaSil}_{(s)} \rightarrow \\
& \mathrm{Cu}(\mathrm{OH})_{2}-\mathrm{NCaSil}_{-} \mathrm{CaSO}_{4(s)} \rightarrow \mathrm{Cu}_{4}(\mathrm{OH})_{6} \mathrm{SO}_{4}
\end{aligned}
$$

Taking into consideration that most of the powder-XRD patterns shown in Figure 3.14 only report the presence of brochantite, it is possible to neglect contributions to peak areas from other substances. Thus, the area underneath the peaks will be directly proportional to the concentration of $B$ in the solid sample. Peaks will grow up to a certain extent until their rate of growth approaches zero. It is possible to obtain the kinetic rate constant for some of the planes by plotting the area of the most intense peaks against time as shown in Figure 3.15.

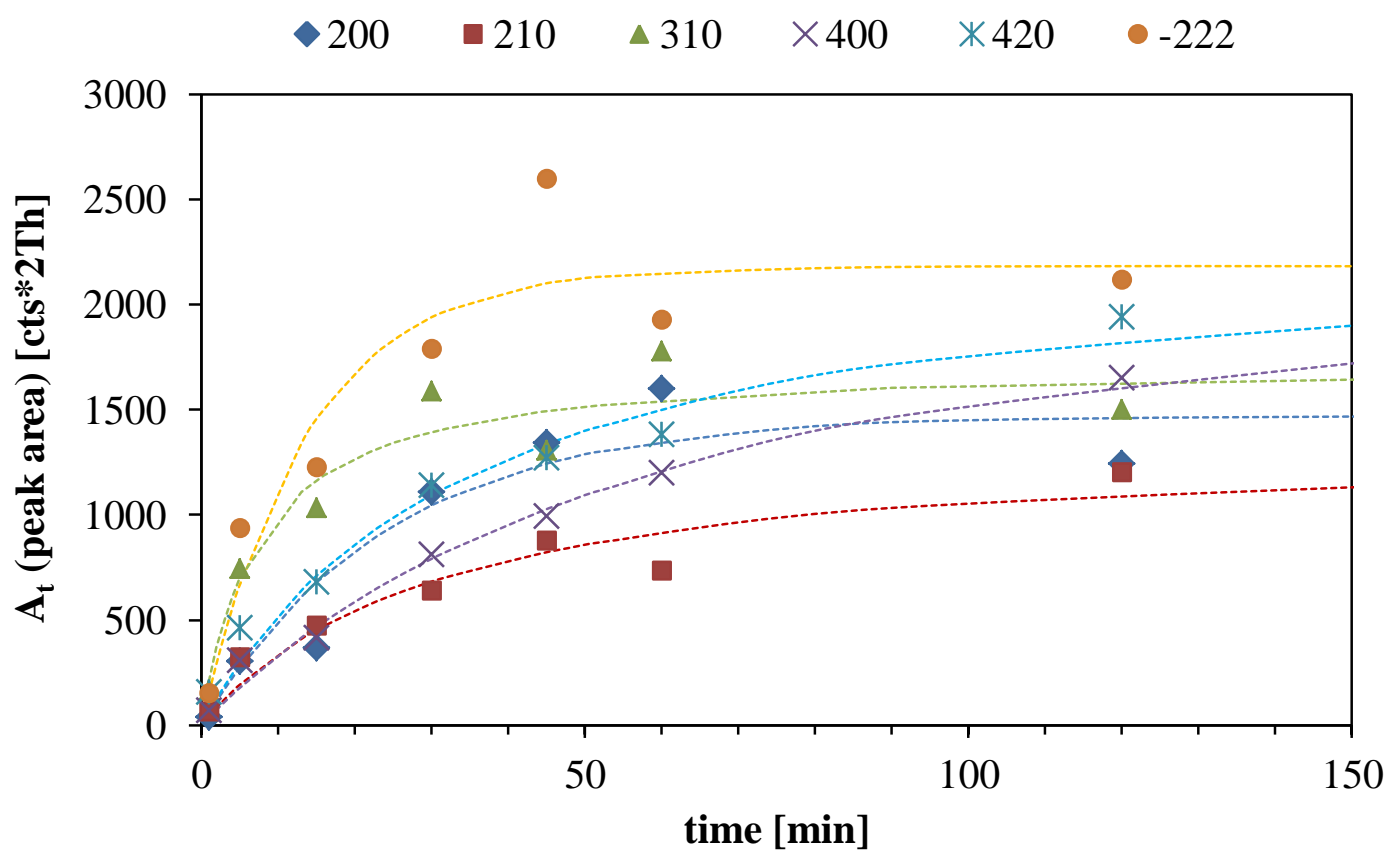

Figure 3.15: Kinetics of plane growth for the most representative peaks in brochantite at $333 \mathrm{~K}$.

The area beneath the peak is directly proportional to the concentration of the crystal being analysed in the sample, hence it is possible to relate these results to pseudo-order adsorption models as they also represent the variation of the concentration on the surface of a certain analyte over time. In Figure 3.15 the experimental data behaves 
identically to an adsorbate in solution being adsorbed onto a solid particle. The concentration on the surface, now represented by the area beneath the peak, grows asymptotically to a certain value at long times, in the same way $q_{t}$ approaches $q_{e}$ over time in Eq. 3.4 and Eq. 3.8. Therefore experimental data was modelled using adaptation of the pseudo-first and pseudo-second order equations for adsorption. The new form is as follows:

$$
A_{t}=A_{e q} \cdot\left(1-e^{-k t}\right)
$$

Variables in eq. 3.21 follow the same logic as the pseudo-first order model for adsorption where $A_{t}$ represents the area at any time $t, A_{e q}$ is the area when the rate of growth is equal to zero (equilibrium), $k$ is the kinetic rate constant, $\mathrm{t}$ is the time, and $k A_{\text {eq }}$ represents the initial rate of growth. In a similar manner the second order equation for adsorption was adapted and the following second order expression, shown in eq. 3.22, was obtained:

$$
A_{t}=\frac{k \cdot\left(A_{e q}\right)^{2} \cdot t}{1+k \cdot A_{e q} \cdot t}
$$

Quantitative data regarding the amount of mass being transferred onto the solid phase cannot be obtained since no standard was placed in the samples. Nevertheless, obtaining the rate constants will help to elucidate which plane is developing faster on the crystal, thus knowing which ions transfer faster to the surface. In the literature most of the time crystal growth is analysed through the variation of the concentration of the reagents in solution which in this case is not valid at most temperatures since multiple crystal structures are formed making the data analysis extremely difficult. Additionally such analysis will not extract kinetic data for individual planes in the crystal. 
Results were fitted to first and second order models, the results are shown in Table 3.6.

Table 3.6: kinetic parameters for plane growth in brochantite crystals at $333 \mathrm{~K}$

\begin{tabular}{|c|c|c|c|c|c|}
\hline \multicolumn{6}{|c|}{ Pseudo-first order } \\
\hline $\begin{array}{l}\text { miller } \\
\text { index }\end{array}$ & $2 \theta$ & $A_{e q}$ & $\boldsymbol{k}$ & $k A_{e q}$ & $r^{2}$ \\
\hline [hkl] & [degrees] & {$\left[\operatorname{cts}^{* 2 \theta}\right]$} & {$\left[\min ^{-1}\right]$} & {$\left[\operatorname{cts}^{\cdot} 2 \theta \min ^{-1}\right]$} & \\
\hline 200 & 13.88 & 1478 & $4.1 \cdot 10^{-2}$ & 60.23 & 0.8792 \\
\hline 420 & 33.45 & 1914 & $2.7 \cdot 10^{-2}$ & 52.00 & 0.9421 \\
\hline-222 & 35.61 & 2181 & $7.3 \cdot 10^{-2}$ & 158.88 & 0.8673 \\
\hline \multicolumn{6}{|c|}{ Pseudo-second order } \\
\hline $\begin{array}{l}\text { miller } \\
\text { index }\end{array}$ & $2 \theta$ & $A_{e q}$ & $\boldsymbol{k}$ & $k\left(A_{e q}\right)^{2}$ & \\
\hline [hkl] & [degrees] & {$[\operatorname{cts} \cdot 2 \theta]$} & $\begin{array}{c}{\left[\operatorname{cts}^{\cdot} \cdot 2 \theta^{-1}\right.} \\
\left.\min ^{-1}\right]\end{array}$ & {$\left[\operatorname{cts} \cdot 2 \theta \min ^{-1}\right]$} & $r^{2}$ \\
\hline 210 & 16.55 & 1391 & $2.3 \cdot 10^{-5}$ & 44.56 & 0.9061 \\
\hline 310 & 22.79 & 1737 & $7.8 \cdot 10^{-5}$ & 235.34 & 0.8944 \\
\hline 400 & 27.96 & 2556 & $5.8 \cdot 10^{-6}$ & 38.07 & 0.9853 \\
\hline
\end{tabular}

Planes 200, 420 and -222 best fit the first order equation; while the remaining planes fit better to a second order (only best fits are collated in Table 3.6). In order to understand why these differences occur, it is important to know which atoms are located on each of the studied planes. Additionally, the fact that certain planes fit to a pseudo-first order equation may be an indication that some are diffusion controlled, while the ones presenting a good correlation to a pseudo-second order equation might limited by the by a chemical reaction. Nonetheless, crystals tend to grow most rapidly at those faces that are more densely packed in the structure [80]. Hence, that should be the case for plane 310. 
c

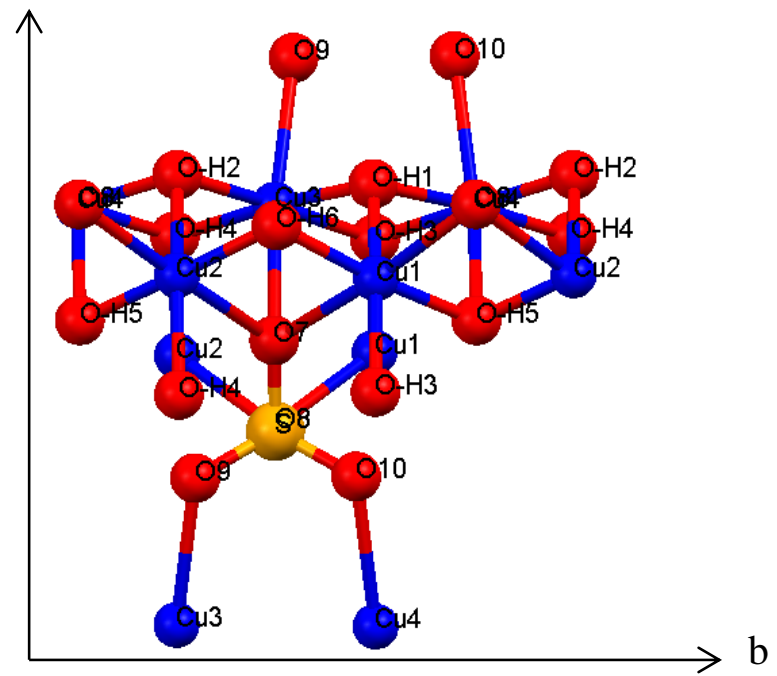

c

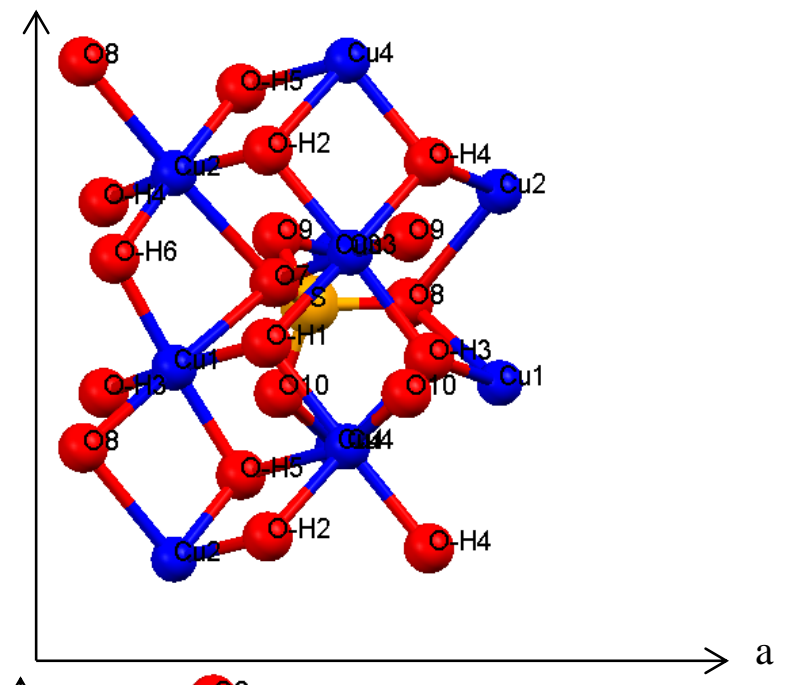

$\mathrm{b}$

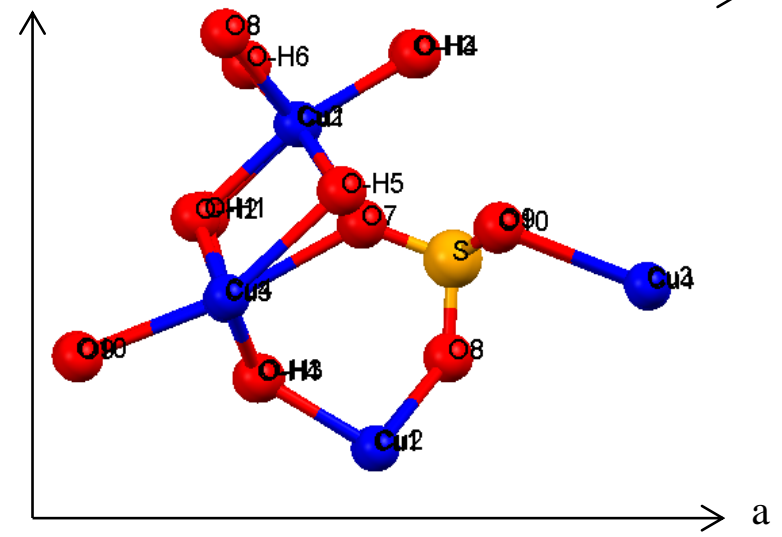

Figure 3.16: Brochantite crystal unit cell drawn using mercury 3.0 software using data obtained in reference [81]. 
Figure 3.17: Atomic contribution to each of the different analysed planes of brochantite using data obtained in reference [81].

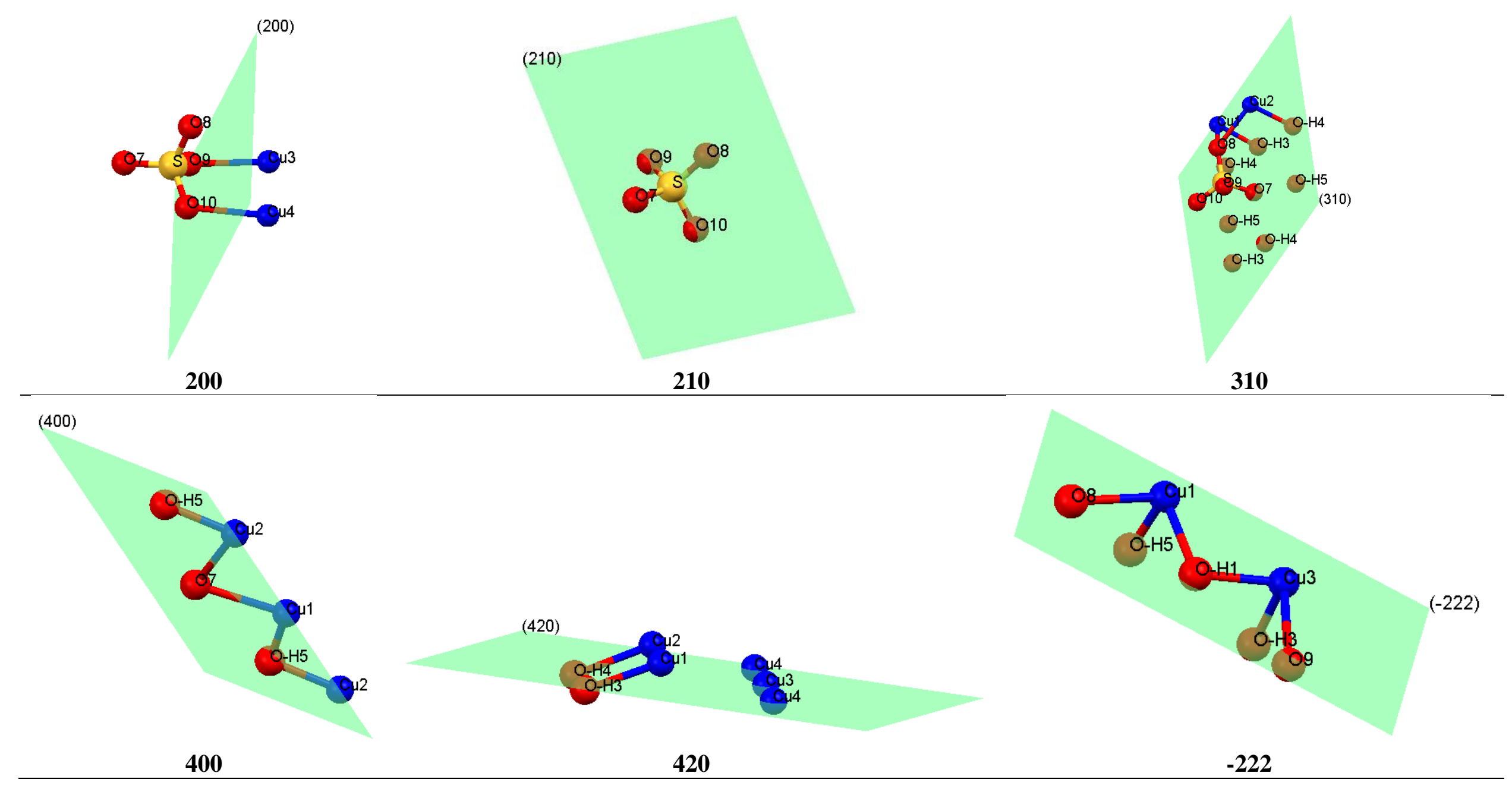


In Figure 3.17 all studied planes are shown in relation to the nearby atoms. Plane 210 and 200 are two of the slowest planes to develop. Coincidentally these planes pass near the sulfate moiety in the unit cell of brochantite. This finding suggests that either the nucleation rate of sulfate to the crystal and/or the relocation of this anion in the crystal lattice may be the rate determining step of the transformation from wroewolfeite and posnjakite into brochantite.

Samples having multiple crystalline phases present a challenge due to overlapping of peaks between substances, thus making quantification of the area very difficult. In order to retrieve useful information about the kinetics of crystal growth a peak with a medium to high intensity needs to be chosen. The peak at $33.46^{\circ} 2 \theta$ was found to uniquely correspond to plane 420 of brochantite. Results for the growth of this plane over time are plotted in Figure 3.18.

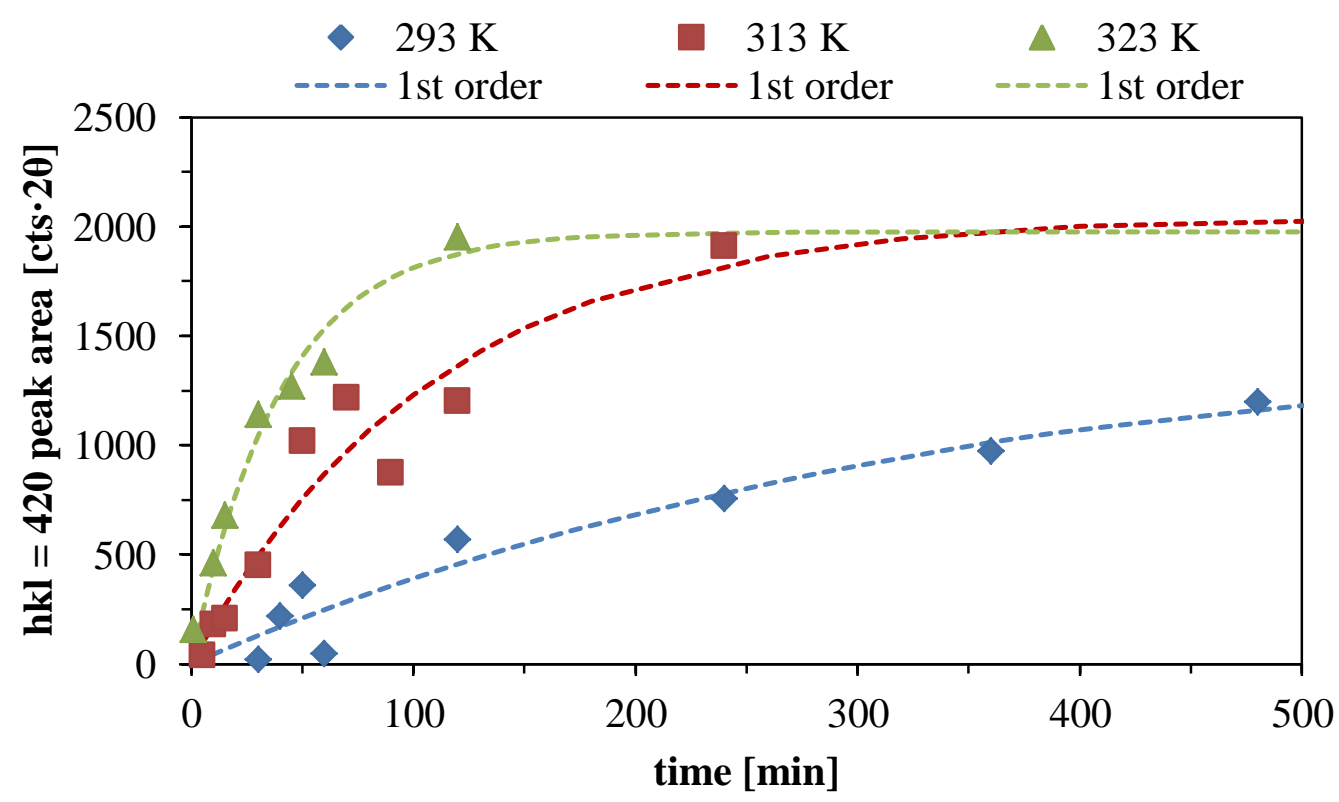

Figure 3.18: Kinetic study on the growth of plane 420 at 293, 313 and $333 \mathrm{~K}$. The experimental data presented on the ordinate represents the peak area of the reflection at $33.46^{\circ} 2 \theta \mathrm{Cu} \mathrm{K}_{\alpha}$ in Figure 3.11, Figure 3.13 and Figure 3.14.

The experimental data fits pseudo-first order kinetics for all studied temperatures. The equilibrium time $t_{e q}$ was arbitrarily set at $0.99 \cdot A_{e q}$. The remaining $1 \%$ is considerably slower and may be neglected. Kinetic parameters for the first order fit are shown in Table 3.7. 
Table 3.7: Pseudo-first order kinetic parameters for the development of plane 420 in Brochantite.

\begin{tabular}{cccccc}
\hline $\begin{array}{c}\mathbf{T} \\
{[\mathbf{K}]}\end{array}$ & $\begin{array}{c}\boldsymbol{A}_{\boldsymbol{e q}} \\
{[\mathbf{c t s} \cdot \mathbf{2 \theta}]}\end{array}$ & $\begin{array}{c}\boldsymbol{t}_{\boldsymbol{e q}} \\
{[\mathbf{m i n}]}\end{array}$ & $\begin{array}{c}\boldsymbol{k}_{\mathbf{4 2 0}} \\
{\left[\mathbf{m i n}^{-\mathbf{1}}\right]}\end{array}$ & $\begin{array}{c}\boldsymbol{k}_{\mathbf{4 2 0}} \cdot \boldsymbol{A}_{\boldsymbol{e q}} \\
{\left[\mathbf{c t s} \cdot \mathbf{2 \theta} \mathbf{~ m i n}^{-\mathbf{1}}\right]}\end{array}$ & $\boldsymbol{r}^{\mathbf{2}}$ \\
\hline 293 & 1568 & 1621 & 0.00287 & 4.50 & 0.9188 \\
313 & 2055 & 507 & 0.00916 & 18.8 & 0.9132 \\
333 & 1914 & 187 & 0.02717 & 52.00 & 0.9684 \\
\hline
\end{tabular}

*Parameters were obtained using Origin 8.0 fitting tool.

Results show that the area underneath the 420 peak tends to a value of approximately $2000 \mathrm{cts} \cdot 2 \theta$ at temperatures higher than $293 \mathrm{~K}$. The kinetic rate constant and the initial growth rate is one order of magnitude larger after an increase in temperature of $40 \mathrm{~K}$ indicating a mild to strong dependence on the temperature. A mass $\sim 1.1 \mathrm{~g}$ of solids were obtained from the experiments when $1 \mathrm{~g}$ of NCaSil was contacted with a $15.7 \mathrm{mmol} \mathrm{Cu}^{2+}$ per $\mathrm{L}$ solution. Therefore a rough estimation tells us that for every 1 mmol of brochantite, under utilized measurement conditions, 1000 cts $2 \theta$ are observed for the peak 420 at the equilibrium. Therefore the initial rate $\left(k A_{e q}\right)$ is in the range of $10^{-3}$ to $10^{-2} \mathrm{mmol} \mathrm{g}^{-1} \mathrm{~min}^{-1}$ for any studied temperature. This value is very important as it makes possible the comparison with previously obtained kinetic data. The value is approximately 3 orders of magnitude smaller than the initial rate of calcium leaching (1.60 $\left.\mathrm{mmol} \mathrm{L}^{-1} \mathrm{~min}^{-1}\right)$ and copper ions uptake $\left(0.870 \mathrm{mmol} \mathrm{g}^{-1} \mathrm{~min}^{-}\right.$ $\left.{ }^{1}\right)^{1}$. Hence, this indicates that crystal formation is the rate determining step towards the formation of the thermodynamical stable compound, brochantite.

In Figure 3.19 an Arrehnius plot was constructed to calculate the activation energy for the formation of plane 420 .

${ }^{1}$ Since $1 \mathrm{~g}$ of NCaSil was used in $1 \mathrm{~L}$ of $\mathrm{Cu}^{2+}$ solution both constants are comparable. 


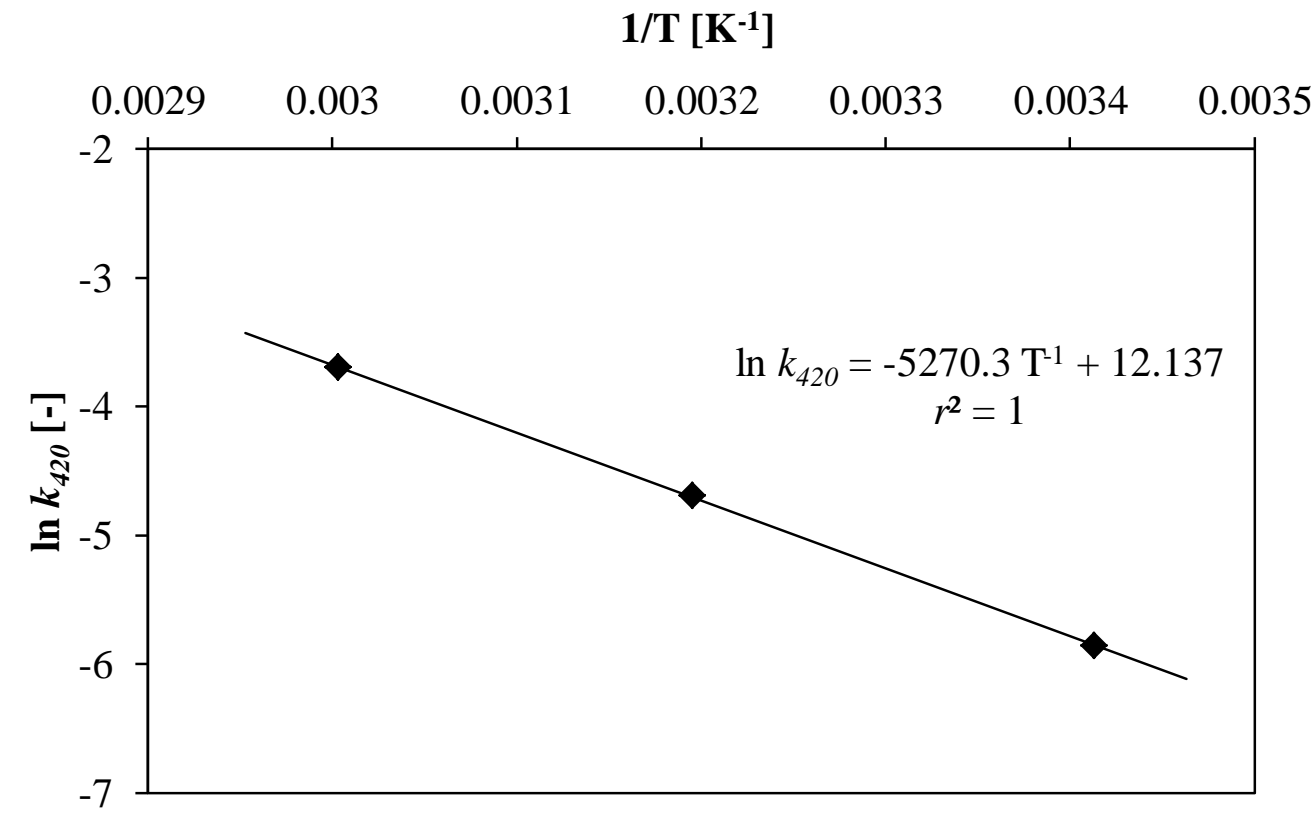

Figure 3.19: Arrhenius plot for the growth of plane 420 of Brochantite using kinetic rate constant reported in Table 3.7.

The slope of the linear regression in Figure 3.19 gives a value for the activation energy of $43.8 \pm 0.3 \mathrm{~kJ} \mathrm{~mol}^{-1}$. Considering that plane 420 forms within the first 15 minutes, even at $293 \mathrm{~K}$, this value is an indication of what magnitude of $E_{a}$ can we expect for other planes. Comparing this result to those previously mentioned in this section for the dehydration of copper sulfate trihydrate $\left(32.6 \mathrm{~kJ} \mathrm{~mol}^{-1}\right)$ both are similar.

It is possible to estimate the temperature needed to form brochantite without forming intermediates by plotting temperature against the time required to reach equilibrium for the development of plane 420 . 


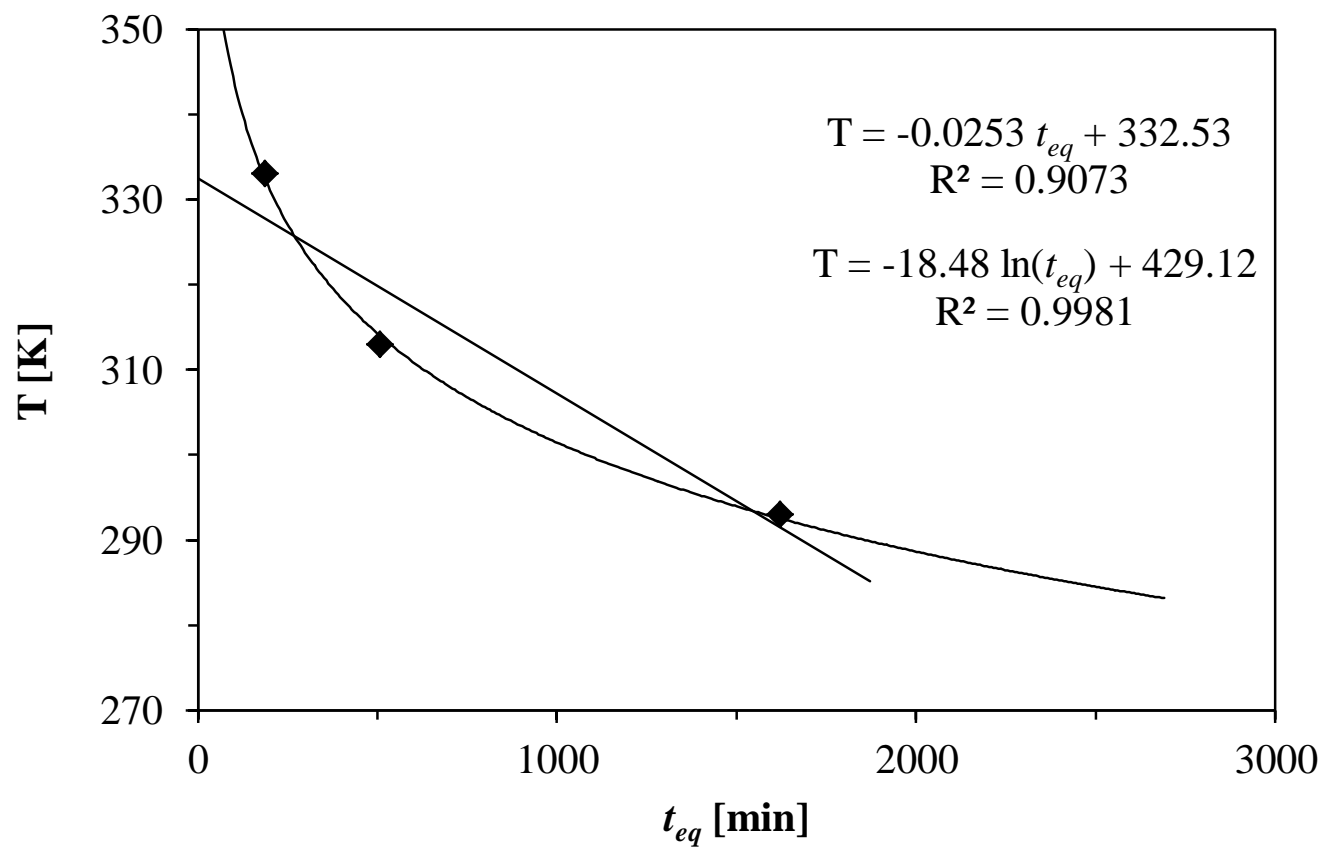

Figure 3.20: Temperature dependence of the time to reach equilibrium for plane 420 .

The intercept of the linear regression shown in Figure 3.20 gives a value of $333 \pm 8 \mathrm{~K}$ which is consistent to the XRD patterns obtained for this same temperature where brochantite forms rapidly. This is an approximation as this trend should be asymptotic to the vertical and horizontal axis since crystals cannot form instantaneously $\left(t_{e q}=0\right)$. Furthermore extremely high temperatures would result in thermal decomposition of the crystal lattice. Nonetheless, at temperatures significantly higher than $333 \mathrm{~K}$ and below the temperature where thermal decomposition of brochantite occurs, it is possible to expect and assume that the mechanism will lead to the direct formation of brochantite without progressing through the intermediates wroewolfeite and posnjakite. 
Different minerals form during the uptake of $\mathrm{Cu}^{2+}$ onto NCaSil. The type depends on concentration, contact time and concentration of the copper sulfate solution. It is possible to propose a general empirical equilibrium equation taking into consideration that posnjakite, wroewolfeite and brochantite possess the same chemical equation $\mathrm{Cu}_{4}(\mathrm{OH})_{6} \mathrm{SO}_{4} \cdot n \mathrm{H}_{2} \mathrm{O}$ with varying amount of waters of crystallization with values of $n$ being 2, 1 and 0 respectively. The empirical equation that describes the reaction would have to take into consideration that NCaSil partially reacts with $\mathrm{Cu}^{2+}$ and $\mathrm{SO}_{4}{ }^{2-}$ resulting in:

- Pure brochantite crystals ( $\mathrm{t}>1440 \mathrm{~min} ; 293 \mathrm{~K})$.

- Monomeric silica and $\mathrm{Ca}^{2+}$ in solution.

- Unreacted sulfate and $\mathrm{Cu}^{2+}$ in solution.

- Amorphous silica.

The reaction mass balance may be written as:

$$
\begin{aligned}
& a \cdot \mathrm{Ca}_{w} \mathrm{Si}_{x}(\mathrm{OH})_{y} \cdot w \cdot \mathrm{H}_{2} \mathrm{O}+b \cdot \mathrm{Cu}^{2+}+c \cdot \mathrm{SO}_{4}{ }^{2-} \rightarrow \\
& d \cdot \mathrm{Cu}_{4}(\mathrm{OH})_{6} \mathrm{SO}_{4(s)}+e \cdot \mathrm{SiO}_{2(s)}+f \cdot \mathrm{SiO}_{2(a q)}+\mathrm{g} \cdot \mathrm{SO}_{4}{ }^{2-}+h \cdot \mathrm{Cu}^{2+}+i \cdot \mathrm{Ca}^{2+}
\end{aligned}
$$

Table 3.8 shows a common reaction in this study where $1 \mathrm{~g}$ of NCaSil was contacted with a concentrated solution $\left(>7.9 \mathrm{mmol} \mathrm{L}^{-1}\right)$ of copper sulfate at temperatures higher than $293 \mathrm{~K}$ for $24 \mathrm{~h}$. Both mass transfer from the bulk of the solution and surface transport of compounds has been reached and formed brochantite as proven by powder-XRD data. 
Table 3.8: Mass balance for the uptake of $\mathrm{Cu}^{2+}$ onto NCaSil

Experimental conditions: $\left[\mathrm{Cu}^{2+}\right]_{\text {initial }} 15.7 \mathrm{mmol} \mathrm{L}^{-1} ; 1 \mathrm{~L}$ solution; $1.0000 \mathrm{~g}$ NCaSil; Temperature > $293 \mathrm{~K}$; reaction time > $24 \mathrm{~h}$

$\mathrm{Cu}_{4}(\mathrm{OH})_{6} \mathrm{SO}_{4}$ corresponds to brochantite

\begin{tabular}{|c|c|c|c|c|c|c|c|c|c|c|c|}
\hline & & $\mathbf{a}^{\cdot} \mathrm{Ca}_{\mathrm{x}} \mathrm{Si}_{\mathrm{y}}(\mathrm{OH})_{\mathrm{w}} \cdot \mathrm{z}\left(\mathrm{H}_{2} \mathrm{O}\right)$ & $\mathbf{b} \cdot \mathbf{C u}^{2+}$ & $\mathrm{c} \cdot \mathrm{SO}_{4}{ }^{2-}$ & $=$ & $\mathrm{d} \cdot \mathrm{Cu}_{4}(\mathrm{OH})_{6} \mathrm{SO}_{4}$ & $\mathrm{e} \cdot \mathrm{SiO}_{2}(\mathrm{~s})$ & $\mathbf{f} \cdot \mathrm{SiO}_{2}$ (in solution) & $\mathrm{g} \cdot \mathrm{SO}_{4}{ }^{2-}$ & $\mathbf{h} \cdot \mathbf{C u}^{2+}$ & $\mathbf{i} \cdot \mathbf{C a}^{2+}$ \\
\hline \multirow{6}{*}{$\overline{\mathrm{O}}$} & $\mathrm{Ca}^{2+}$ & $a \cdot x$ & & & $=$ & & & & & \multirow{6}{*}{$\mathrm{h}$} & $\mathrm{i}$ \\
\hline & Si & $a \cdot y$ & & & $=$ & & $\mathrm{e}$ & $\mathrm{f}$ & & & \\
\hline & $\mathrm{Cu}^{2+}$ & & $\mathrm{b}$ & & $=$ & $4 d$ & & & & & \\
\hline & $\mathrm{SO}_{4}{ }^{2-}$ & & & $\mathrm{c}$ & $=$ & $\mathrm{d}$ & & & $\mathrm{g}$ & & \\
\hline & $\mathbf{O}$ & $\mathrm{a} \cdot \mathrm{w}+\mathrm{z}$ & & & $=$ & $6 \mathrm{~d}$ & $2 \mathrm{e}$ & $2 \mathrm{f}$ & & & \\
\hline & $\mathbf{H}$ & $a \cdot w+2 z$ & & & $=$ & $6 d$ & & & & & \\
\hline
\end{tabular}

Table 3.9: Stoichiometric coefficients for Table 3.8

\begin{tabular}{|c|c|c|c|c|c|c|c|c|c|}
\hline Values obtained from: & $\begin{array}{c}\text { Table } 1.3 \\
1.00 \mathrm{~g} / \mathrm{M}_{\mathrm{NCaSil}}\end{array}$ & Initi & unt & $(b-h) / 4$ & $\mathbf{a} \cdot \mathbf{x}-\mathbf{f}$ & Table 3.4 & c-d & $\begin{array}{c}\text { Table } 3.2 \\
\left(15.7-q_{e}\right), 293 \mathrm{~K}\end{array}$ & Table 3.3 \\
\hline & $\mathbf{a}$ & $\mathbf{b}$ & $\mathbf{c}$ & d & $\mathbf{e}$ & $\mathbf{f}$ & g & $\mathbf{h}$ & $\mathbf{i}$ \\
\hline mmol & 7.2 & 15.7 & 15.7 & 2.3 & 14.4 & 3.7 & 13.4 & 6.5 & 6.8 \\
\hline
\end{tabular}


The equation depicted in Table 3.8 is based on experimental observation that has been discussed throughout Chapter 3 , which assumes that $1 \mathrm{~L}$ of a $15.7 \mathrm{mmol} \mathrm{L}^{-1}$ $\mathrm{Cu}^{2+}$ solution was contacted with $1 \mathrm{~g}$ of $\mathrm{NCaSil}$ at $293 \mathrm{~K}$ in a stirred reactor. The stoichiometric coefficients for such reaction are shown in Table 3.8 and in Table 3.9. The stoichiometric coefficients correspond to the same amounts used in the experiments throughout chapter 3. Values for $a$ were obtained from Table 1.3 using a medium range molar mass. The values of $b$ and $c$ correspond to the initial mmol added into the reaction. The values of $d, e$ and $g$ were obtained by the mathematical operations shown in Table 3.9. The value of $f$ corresponds to that obtained in section 3.2.4 in the study of the release of monomeric silica at $293 \mathrm{~K}$. The value of $h$ is the remaining $\mathrm{Cu}^{2+}$ concentration in solution at $293 \mathrm{~K}$. The value of $i$ is obtained from Table 3.3 and corresponds to amount of $\mathrm{Ca}^{2+}$ present in solution at equilibrium.

It is possible to verify the empirical results by subtracting the final amount of mmol of each compound from the initial amount $(\Delta \mathrm{mmol})$, The result of such mathematical operation is shown in Table 3.10 .

Table 3.10: Overall mass balance result

\begin{tabular}{|c|c|c|c|}
\hline Analyte & $\begin{array}{c}\text { Low-range } \\
\mathbf{M}_{\text {NCaSil }} \\
\mathrm{w}=\mathbf{0 . 5} ; \mathrm{x}=\mathbf{2 . 0 5} \\
\mathbf{y}=\mathbf{0} ; \mathrm{z}=\mathbf{1 . 4}\end{array}$ & $\begin{array}{c}\text { Mid-range } \\
\text { M }_{\mathrm{NCaSil}} \\
\mathrm{w}=\mathbf{0 . 7 5} ; \mathrm{x}=\mathbf{2 . 5 3} \\
\mathbf{y}=\mathbf{0 . 2 5} ; \mathrm{z}=\mathbf{1 . 9}\end{array}$ & $\begin{array}{c}\text { High-range } \\
M_{\mathrm{NCaSil}} \\
\mathrm{w}=1 ; \mathrm{x}=\mathbf{3} \\
\mathrm{y}=\mathbf{0 . 5} ; \mathrm{z}=\mathbf{2 . 5}\end{array}$ \\
\hline & $\Delta \mathrm{mmol}$ & $\Delta \mathrm{mmol}$ & $\Delta \mathrm{mmol}$ \\
\hline $\mathrm{Ca}^{2+}$ & 1.9 & 1.4 & 1.2 \\
\hline $\mathrm{Si}$ & 0.0 & 0.0 & 0.0 \\
\hline $\mathrm{Cu}^{2+}$ & 0.0 & 0.0 & 0.0 \\
\hline $\mathrm{SO}_{4}{ }^{2-}$ & 0.0 & 0.0 & 0.0 \\
\hline $\mathrm{O}$ & 52.3 & -5.4 & 42.2 \\
\hline $\mathrm{H}$ & 11.0 & -15.2 & 6.0 \\
\hline
\end{tabular}

From the results shown Table 3.10, $\mathrm{Ca}^{2+}$ could have been quantified in excess or under estimated in molecular formula of NCaSil. On the other hand, the mass balance for $\mathrm{Cu}^{2+}, \mathrm{Si}$ and sulfate fits closely.

After analysing the mass balance a simplified empirical equation for the uptake of $\mathrm{Cu}^{2+}$ may be stated using the empirical formula of NCaSil with arbitrarily set values to a mid-range content of constituents. The formula is shown in Eq. 3.24. 
$72 \mathrm{Ca}_{0.75} \mathrm{SiO}_{2.53}(\mathrm{OH})_{0.25} \cdot 1.9 \mathrm{H}_{2} \mathrm{O}+157 \cdot \mathrm{Cu}^{2+}+157 \mathrm{SO}_{4}{ }^{2-} \rightarrow$

$23 \mathrm{Cu}_{4}(\mathrm{OH})_{6} \mathrm{SO}_{4(s)}+35 \mathrm{SiO}_{2(s)}+\cdot 37 \mathrm{SiO}_{2(a q)}+134 \mathrm{SO}_{4}{ }^{2-}+65 \mathrm{Cu}^{2+}+68 \mathrm{Ca}^{2+}$

(eq. 3.24)

Considering that the molar mass was calculated arbitrarily from values reported in McFarlane's work [36] and that NCaSil offers a range of content of the different species, discrepancies in the amounts reported in Table 3.10 for calcium, oxygen and hydrogen content in the reagents and products may be solved by the modifying the original formula within the reported range. The reaction may be finally balanced by the addition of 3.2 moles of water and adjusting calcium and oxygen content in NCaSil from 0.75 to 0.94 and from 2.53 to 2.25 , respectively. Eq. 3.25 shows the balanced equation for the reaction stated in Eq. 3.23 using stoichiometric values shown in Table 3.8 and Table 3.9.

$7.2 \mathrm{Ca}_{0.94} \mathrm{SiO}_{2.25}(\mathrm{OH})_{0.5} \cdot 1.9 \mathrm{H}_{2} \mathrm{O}+15.7 \cdot \mathrm{Cu}^{2+}+15.7 \mathrm{SO}_{4}{ }^{2-}+3.2 \mathrm{H}_{2} \mathrm{O} \rightarrow$

$2.3 \mathrm{Cu}_{4}(\mathrm{OH})_{6} \mathrm{SO}_{4(s)}+3.5 \mathrm{SiO}_{2(s)}+3.7 \mathrm{SiO}_{2(a q)}+6.8 \mathrm{Ca}^{2+}+6.5 \mathrm{Cu}^{2+}+13.4 \mathrm{SO}_{4}{ }^{2-}$ (eq. 3.25)

The resulting NCaSil formula $\left(\mathrm{Ca}_{0.94} \mathrm{SiO}_{2.25}(\mathrm{OH})_{0.5} \cdot 1.9 \mathrm{H}_{2} \mathrm{O}\right)$ is still inside the range reported in Table 1.3. This should be taken as an approximation to the NCaSil prepared during this work.

Apparently the reaction shown in Eq. 3.25 depicts a 1:1 ion exchange process between $\mathrm{Cu}^{2+}$ and $\mathrm{Ca}^{2+}$, but further experiments are required to confirm this. 


\subsubsection{SEM and EDS study on the kinetics of $\mathrm{Cu}^{2+}$ adsorption and crystal growth}

In the quest to find proof of a stepwise mechanism for the sorption of copper a kinetic study using SEM and EDS for the uptake of copper by NCaSil from solution was undertaken. In a previous study using similar conditions, Cairns [37] analysed SEM images of $\mathrm{Cu}$-free $\mathrm{NCaSil}$ and at times corresponding the equilibrium. In this study, the reaction was stopped at different times in order to observe how the formation of the crystals occurs. Additionally this will help to see if there was any site specificity for the adsorption of $\mathrm{Cu}^{2+}$. Figure 3.21 to Figure 3.28 show the crystal growth from $t=1 \mathrm{~min}$ to $t=120 \mathrm{~min}$.

Taking into consideration the XRD patterns obtained in Figure 3.13 the observation of the development of posnjakite (P) and brochantite (B) was pursued. As revealed from XRD data posnjakite is expected to be the first crystal to develop and should appear in the early stages of the reaction.

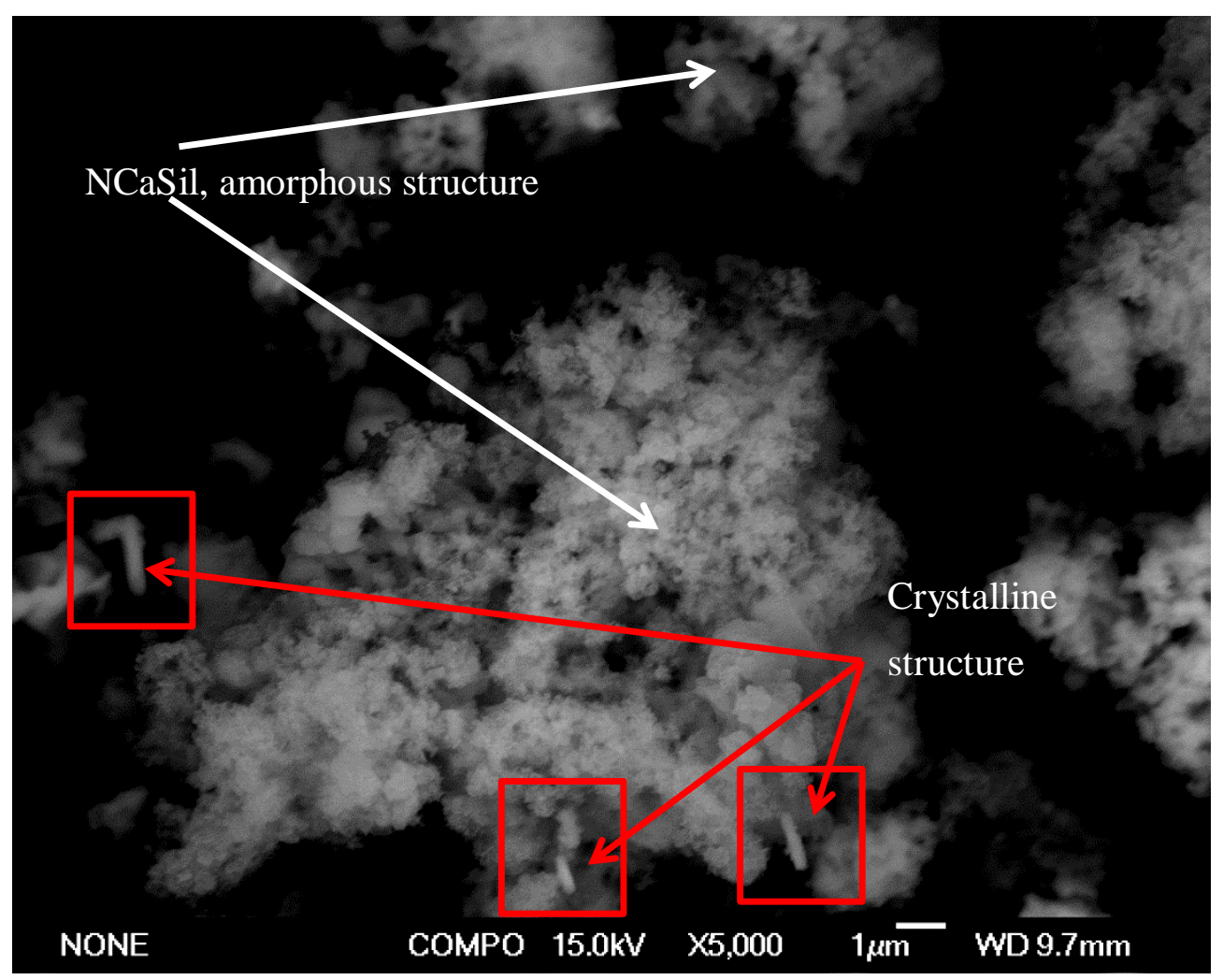

Figure 3.21: SEM micrograph on backscatter mode for $t=1 \mathrm{~min}$. Experimental conditions: $\left[\mathrm{Cu}^{2+}\right]_{\text {initial }} 15.7 \mathrm{mmol} \mathrm{L}^{-1}$; adsorbent dosage $1 \mathrm{~g} \mathrm{~L}^{-1}$; temperature of the reaction $313 \mathrm{~K}$.

Figure 3.21 shows a representative image of the product after the first minute of the reaction. Crystalline structures are marked inside red boxes while amorphous structures are pointed with white arrows. After one minute of reaction a few crystals have rapidly developed on the surface of the NCaSil, with sizes ranging 0.5 to 1 micrometre with a preferential growth in one dimension. It is most likely that this 
crystal corresponds to posnjakite considering the XRD patterns in Figure 3.13 for this same temperature.

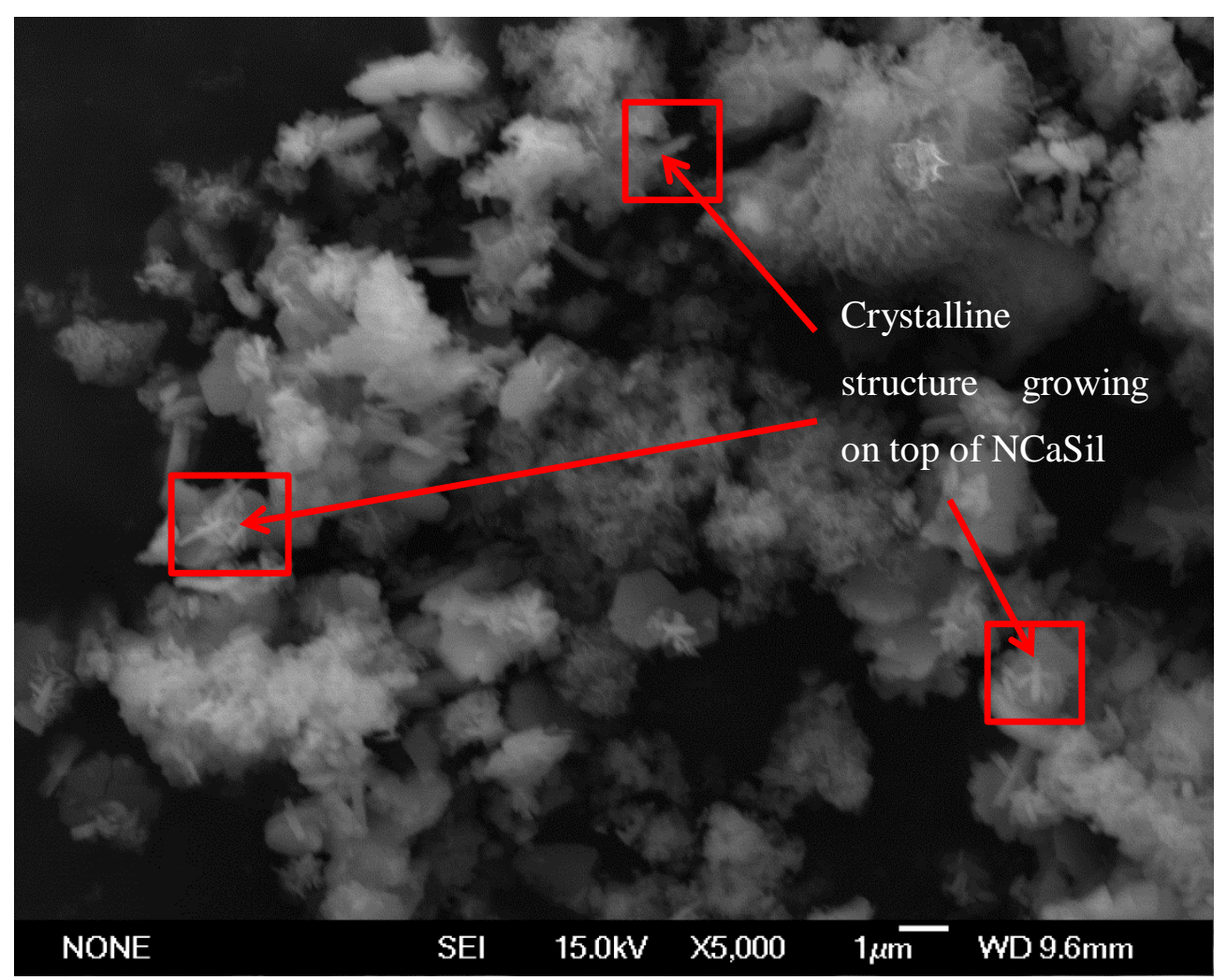

Figure 3.22, SEM micrograph for $t=5 \mathrm{~min}$. Experimental conditions: $\left[\mathrm{Cu}^{2+}\right]_{\text {initial }} 15.7 \mathrm{mmol} \mathrm{L}^{-1}$; adsorbent dosage $1 \mathrm{~g} \mathrm{~L}^{-1}$; temperature of the reaction $313 \mathrm{~K}$.

Figure 3.22 shows that 5 minutes into the reaction more crystals have developed on the surface of NCaSil. Crystalline structures appear to be leaning on the surface of the NCaSil rather than growing from the surface. Further analyses are needed to understand whether crystals are growing from solution and subsequently being deposited on the surface or copper in solution is using NCaSil as a substrate for crystal growth.

In order to prove the latter, a SEM image and EDS mapping were undertaken on a sample after $t=15 \mathrm{~min}$ corresponding to the point where approximately half of the loading capacity $\left(\approx 5 \mathrm{mmol} \mathrm{g}{ }^{-1}\right)$ of $\mathrm{NCaSil}$ has been reached and posnjakite is the predominant crystal structure. The SEM and EDS images for this stage are shown in Figure 3.23 and Figure 3.24. In Figure 3.23 white boxes show the area where NCaSil fresh surface is still exposed to the solution. EDS mapping to the same area is shown in Figure 3.24, where Si presence is represented in red in both overlapped and standalone image. Moreover, the amorphous region is pointed out with yellow arrow and will be studied using EDS in order to observe whether it forms amorphous 
copper hydroxide on the surface of NCaSil as an intermediate in the reaction to form brochantite.

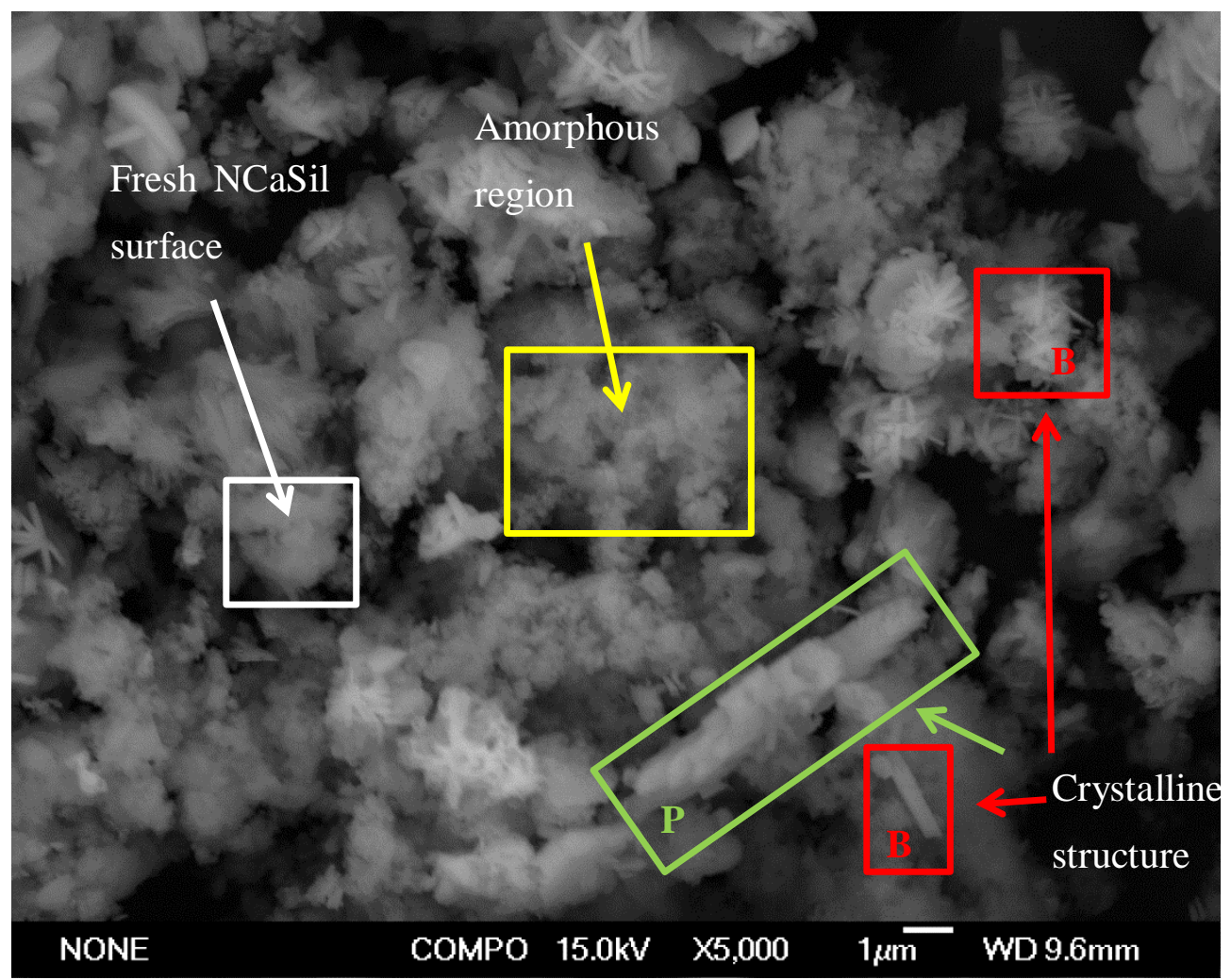

Figure 3.23: SEM micrograph on backscatter mode for $t=10 \mathrm{~min}$. Experimental conditions: $\left[\mathrm{Cu}^{2+}\right]_{\text {initial }} 15.7 \mathrm{mmol} \mathrm{L}^{-1}$; adsorbent dosage $1 \mathrm{~g} \mathrm{~L}^{-1}$; temperature of the reaction $313 \mathrm{~K}$.
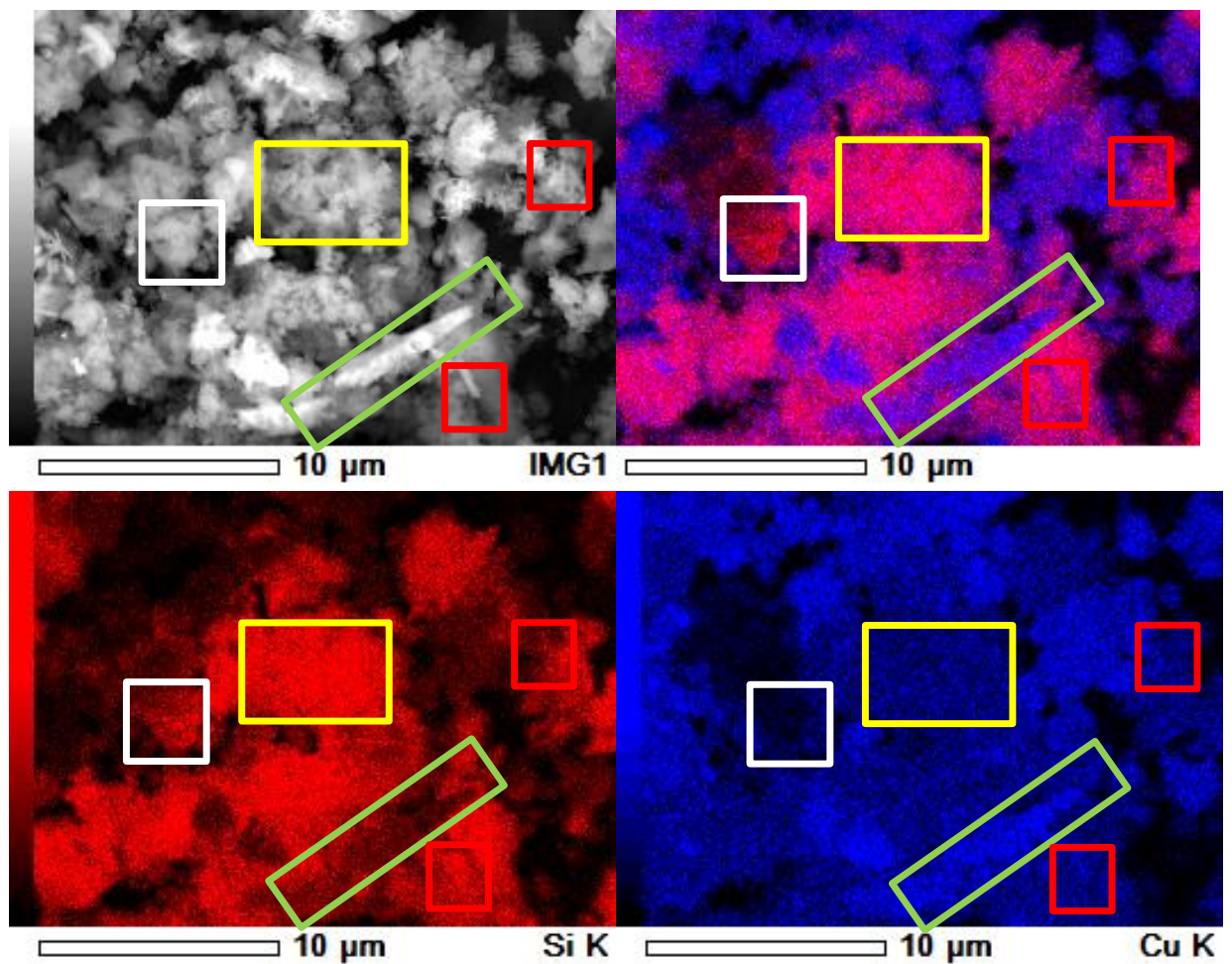

Figure 3.24: SEM image and EDS mapping of Figure 3.23. Si and $\mathrm{Cu}$ are shown in red and blue respectively. Experimental conditions: $\left[\mathrm{Cu}^{2+}\right]_{\text {initial }} 15.7 \mathrm{mmol} \mathrm{L}^{-1}$; adsorbent dosage $1 \mathrm{~g} \mathrm{~L}^{-1}$; temperature of the reaction $313 \mathrm{~K}$. 
The area inside the yellow box has both $\mathrm{Cu}$ and $\mathrm{Si}$ as depicted in the standard (gray coloured) SEM image and the overlapped (pink/red/blue coloured) EDS mapping. This zone appears as pink in the overlapped image. This zone has an amorphous nature since no ordered morphology can be seen in contrast to the well-defined prism structures shown on previous figures.

Two different crystalline structures may be observed enclosed in red and green boxes in Figure 3.23 (please refer to Figure 3.12 to see the differences between posnjakite and brochantite). The structure located within the red box is similar to those seen in previous SEM images, with prisms in the range of 1 micrometre in length. It is most likely that this crystal corresponds to brochantite considering the morphology exhibited in Figure 3.12. The crystalline structure enclosed in green is approximately 5 times longer. This structure resembles either wroewolfeite or posnjakite compared to crystals shown in Figure 3.12. Considering the temperature and the time when the sample was collected during the experiment it is most likely to be posnjakite.

In Figure 3.24 it can be observed that the places where the crystalline structures are located in the image are almost $\mathrm{Si}$-free zones on the EDS maps while $\mathrm{Cu}$ is covering a larger surface. This could imply that the crystals may have detached from the surface of the silicate. Other reasons for this region with low presence of Si could be shielding of the underlying structure by the crystal. Nevertheless, large portions of the material still resemble the initial structure NCaSil, shown in Figure 1.1, suggesting that the reaction has not yet reached equilibrium. 


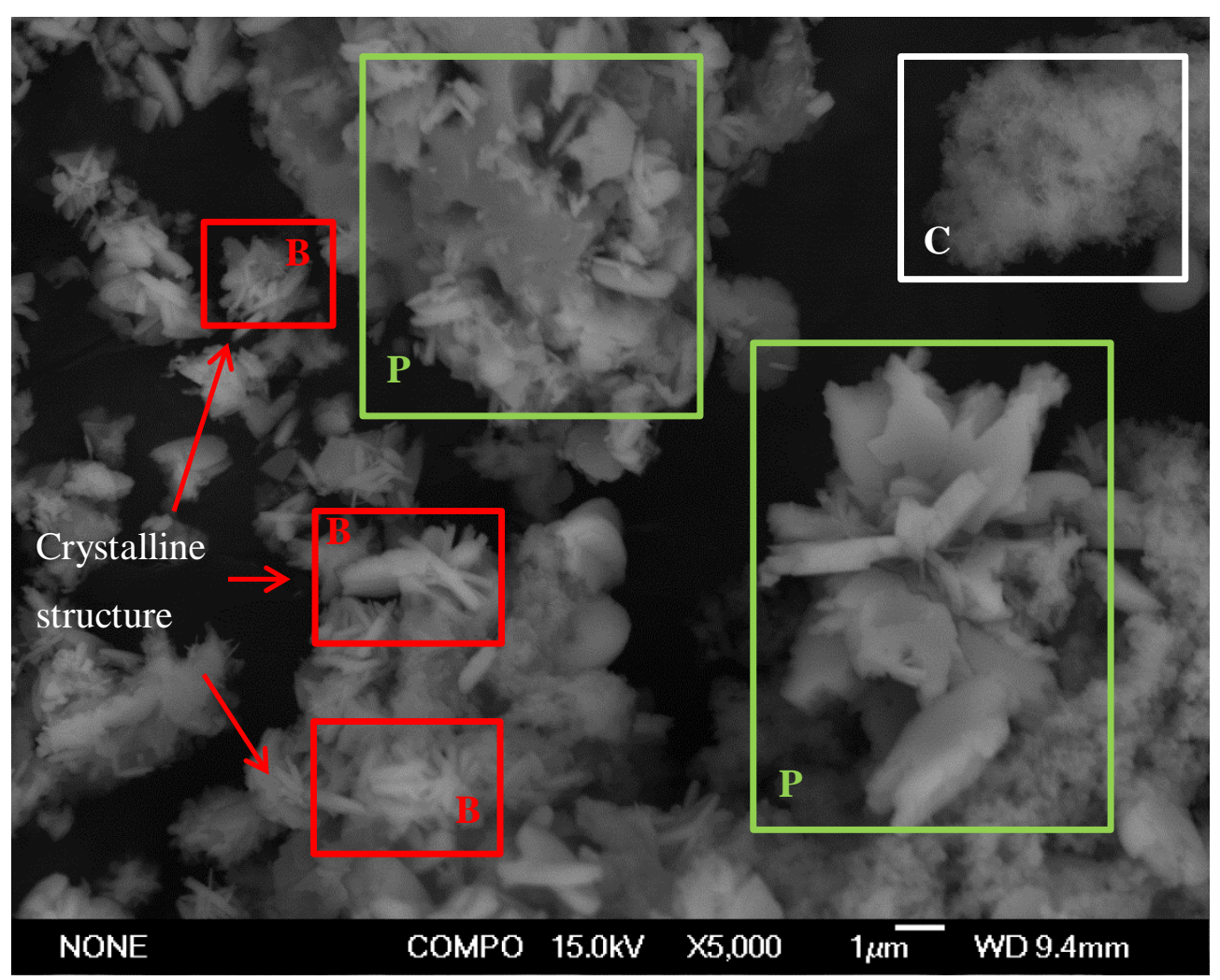

Figure 3.25: SEM micrograph on backscatter mode for $t=15 \mathrm{~min}$. Experimental conditions: $\left[\mathrm{Cu}^{2+}\right]_{\text {initial }} 15.7 \mathrm{mmol} \mathrm{L}^{-1}$; adsorbent dosage $1 \mathrm{~g} \mathrm{~L}^{-1}$; temperature of the reaction $313 \mathrm{~K}$.

Figure 3.25 shows a representative image of the solid phase of the reaction after 15 minutes of starting the experiment. Several features from previous figures may still be observed here, although crystals have grown considerably larger compared to the features found in the sample taken at $t=10 \mathrm{~min}$. This is consistent with fact that the mass transport of $\mathrm{Cu}^{2+}$ onto the solid surface still hasn't reach the equilibrium as shown in Figure 3.3. Enclosed in a white box is a remaining portion of NCaSil which still resembles its initial features with honeycomb type morphology as shown in Figure 1.1. The two types of crystals shown in Figure 3.23 appear again but with the difference that brochantite crystals are approximately 50\% larger in all dimensions than those shown in the previous SEM image. This is in agreement with the XRD patterns in Figure 3.13 showing the development of brochantite crystals as time elapses. 


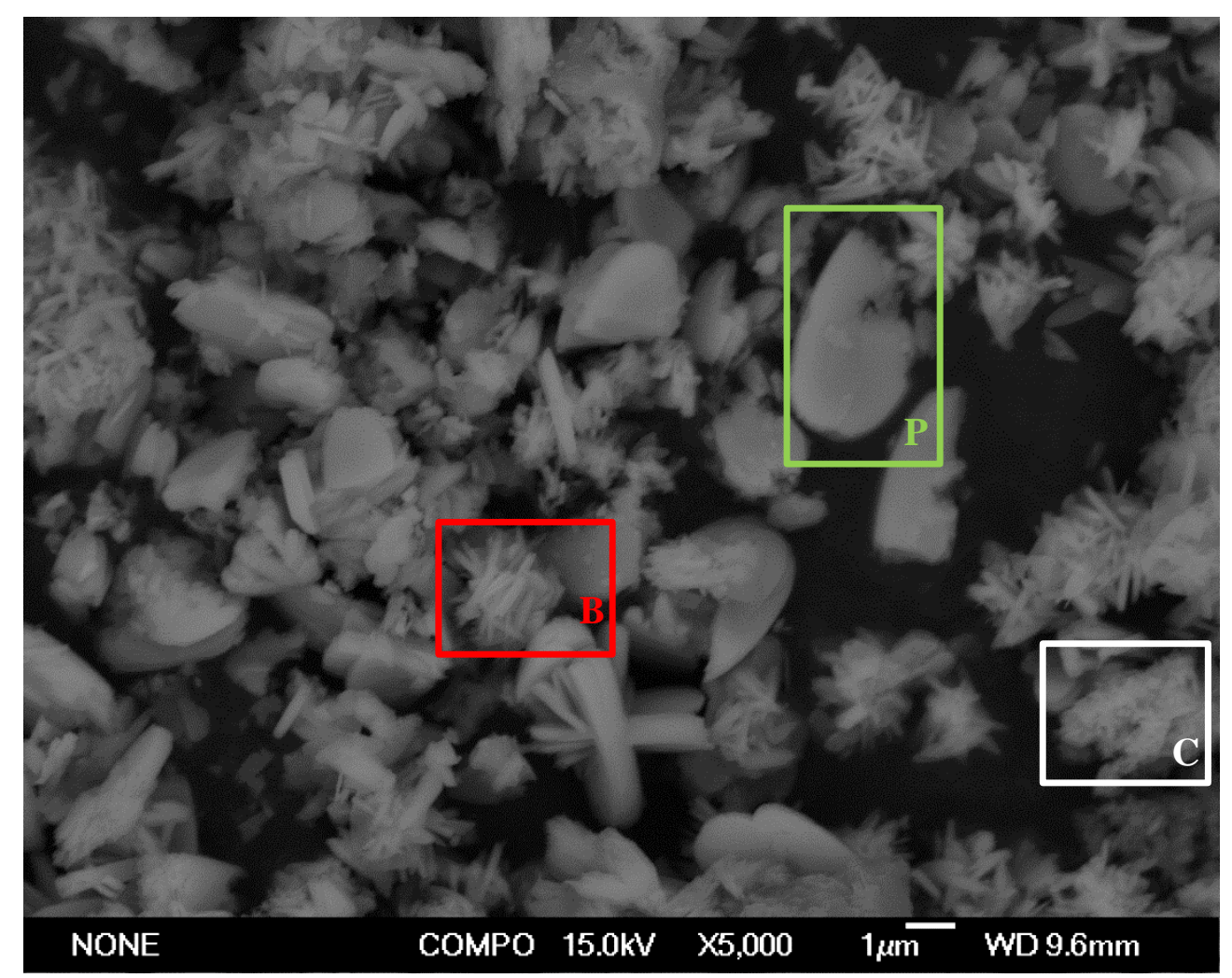

Figure 3.26: SEM micrograph on backscatter mode for $t=30 \mathrm{~min}$. Experimental conditions: $\left[\mathrm{Cu}^{2+}\right]_{\text {initial }} 15.7 \mathrm{mmol} \mathrm{L}^{-1}$; adsorbent dosage $1 \mathrm{~g} \mathrm{~L}^{-1}$; temperature of the reaction $313 \mathrm{~K}$.

Figure 3.26 shows an SEM image of a sample taken after 30 minutes into the reaction. It is clear that most of the sample corresponds to the posnjakite (green box) and brochantite (red box) crystals. This is consistent with the results obtained by XRD where brochantite is becoming the predominant phase at this time of the experiment and $\mathrm{NCaSil}$ and calcium sulfate patterns shrink into the background. On the right bottom corner inside a white box a small portion resembling the initial NCaSil structure still may be observed. Posnjakite crystal size ranges from 3 to $5 \mu \mathrm{m}$, while brochantite crystals are in range of 0.5 to $2 \mu \mathrm{m}$. 


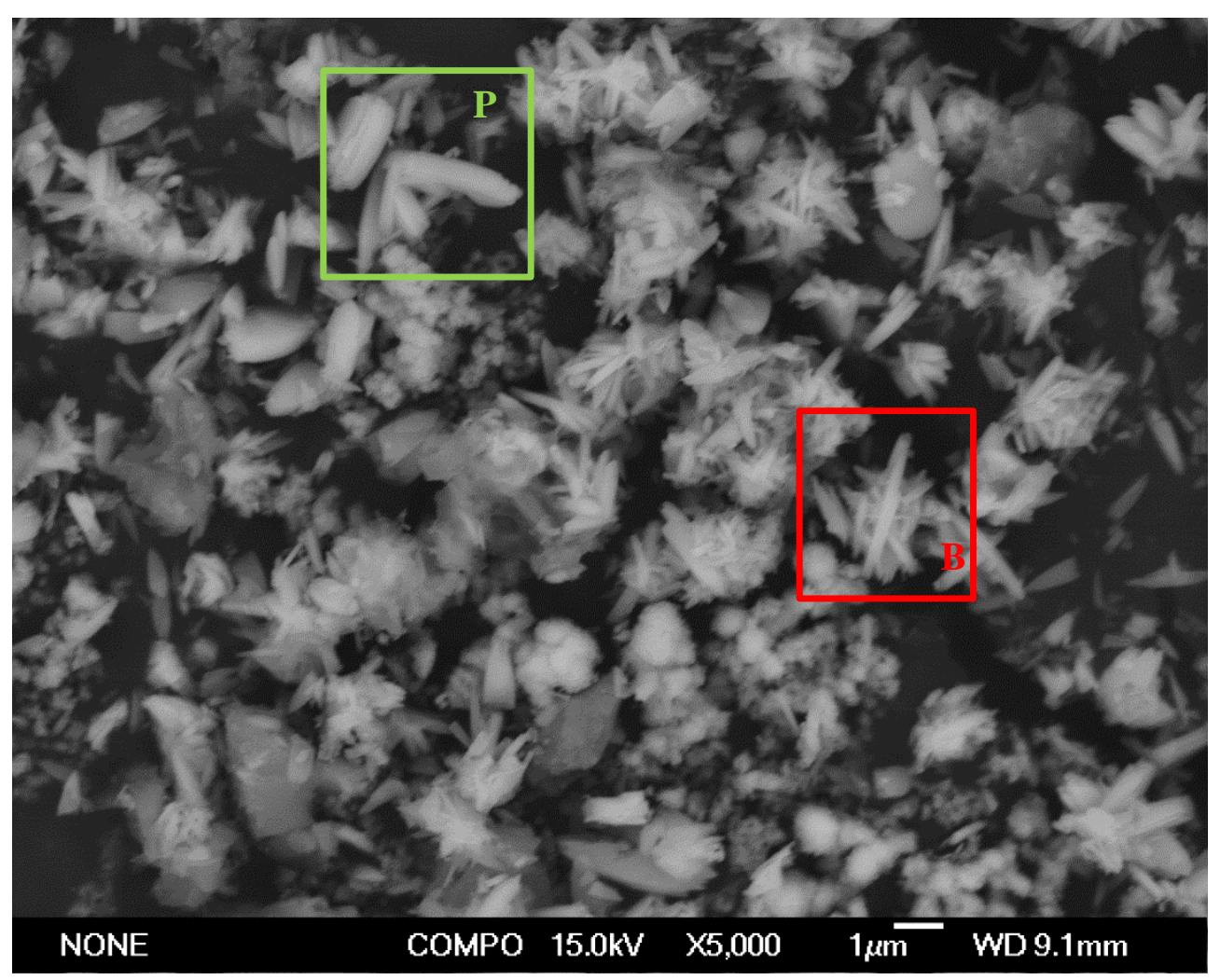

Figure 3.27: SEM micrograph on backscatter mode for $t=60 \mathrm{~min}$. Experimental conditions: $\left[\mathrm{Cu}^{2+}\right]_{\text {initial }} 15.7 \mathrm{mmol} \mathrm{L}^{-1}$; adsorbent dosage $1 \mathrm{~g} \mathrm{~L}^{-1}$; temperature of the reaction $313 \mathrm{~K}$.

After 60 minutes crystal structures of posnjakite and brochantite can still be observed in Figure 3.27. Brochantite crystals (red box) have grown larger in length reaching approximately $3 \mu \mathrm{m}$ in length while posnjakite crystals (green box) in this SEM image appear to be smaller than in previous samples. 


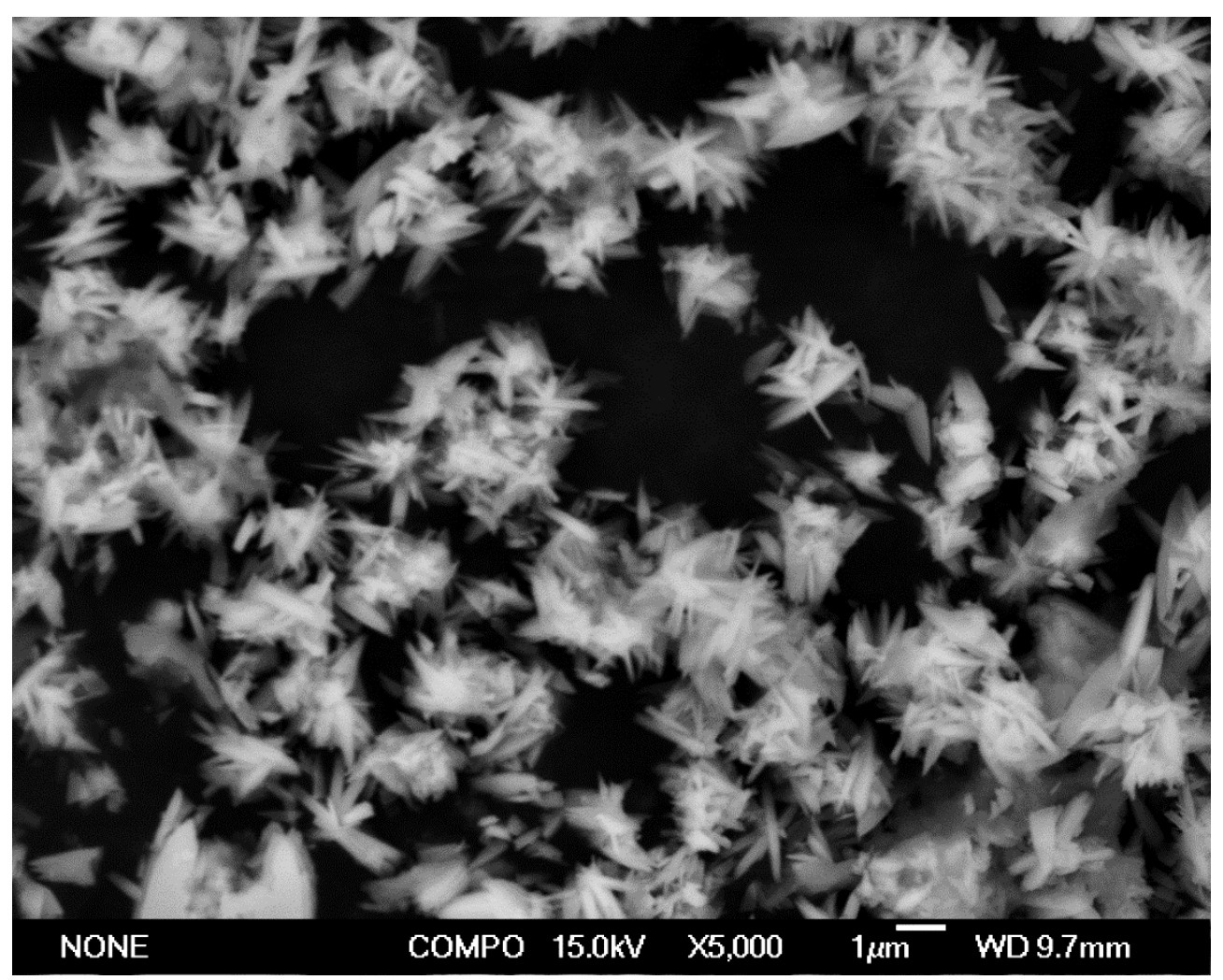

Figure 3.28: SEM micrograph on backscatter mode for $t=120 \mathrm{~min}$. Experimental conditions: $\left[\mathrm{Cu}^{2+}\right]_{\text {initial }} 15.7 \mathrm{mmol} \mathrm{L}^{-1}$; adsorbent dosage $1 \mathrm{~g} \mathrm{~L}^{-1}$; temperature of the reaction $313 \mathrm{~K}$.

Finally, after 120 minutes brochantite is the predominant crystal. This is consistent with what was obtained from the powder-XRD analysis of this sample in Figure 3.13 where brochantite is the main feature. Crystals are apparently similar in size ranging from 1 to $3 \mu \mathrm{m}$ in length. Considering XRD results the sample composition remains constant from this point onwards. Hence, there should be only brochantite in the samples collected later on in the experiment. It is noteworthy that no NCaSil is observable in Figure 3.28.

Overall it appears that crystal growth follow-up using SEM is consistent with the results obtained using powder-XRD. Furthermore, the idea of crystals forming from solution at mild temperatures may be disregarded as it was proved by SEM and EDS that $\mathrm{Cu}$ is present over an amorphous zone rich in $\mathrm{Si}$ as shown in Figure 3.24 suggesting the formation of copper hydroxide. 


\subsection{Study of the equilibrium of the adsorption of $\mathrm{Cu}^{2+}$ onto NCaSil}

If both adsorbate and sorbent are left for a sufficient time the sorption process will reach equilibrium. This happens when both forward (sorption) and backward (desorption) reactions reach the same rate. In the literature several mathematical equations have been reported that help to model the equilibrium of the sorption, but many fail to give useful information as they do not possess strong thermodynamic explanation. In this study the Langmuir, Freundlich and Temkin models will be examined since they give a better understanding of how the adsorbate covers the surface of a sorbent.

\subsubsection{Langmuir Isotherm}

Application of the Langmuir model [82] assumes that the sorption occurs in the form of a monolayer on a homogeneous surface. Thermodynamically this could be understood as the enthalpy of the sorption process (heat of adsorption) being constant as the surface coverage increases. Assuming that the process is represented by eq. 3.26 the rate of change of surface coverage due to the sorption is proportional to the equilibrium concentration of the adsorbate in solution $C_{e}$ and the number of vacant sites $(1-\theta)$. Where $\theta$ is the fractional coverage of the surface defined as the number of sorption sites occupied over the number of sorption sites available.

$$
\frac{d \theta}{d t}=k_{a} C_{e}(1-\theta)
$$

The rate of change of $\theta$ due to desorption is proportional to the number of species adsorbed, therefore,

$$
\frac{d \theta}{d t}=-k_{d} \theta
$$

At the equilibrium both rates are equal and,

$$
\theta=\frac{K_{L} C_{e}}{1+K_{L} C_{e}}
$$

With $K_{L}=k_{a} / k_{d}$ representing the equilibrium constant of the process. Expressing $\theta$ as $q_{e} / q_{m}$ allows the rearrangement of the Langmuir equation to its most common form,

$$
q_{e}=\frac{q_{m} K_{L} C_{e}}{1+K_{L} C_{e}}
$$


where $q_{e}$ is the amount of adsorbate in mmol per $\mathrm{g}$ of sorbent at the surface and $q_{m}$ is the maximum loading capacity of the sorbent for the same adsorbate in mmol per $\mathrm{g}$ of sorbent. The equation can either be linearized or computed through iteration to obtain the maximum load capacity and the equilibrium constant.

The isotherm may also be represented in a linear form to ease calculations. Where $q_{m}$ is obtained from the slope and $K_{L}$ from the intercept in eq. 3.30 .

$$
\frac{C_{e}}{q_{e}}=\frac{C_{e}}{q_{m}}+\frac{1}{K_{L} q_{m}}
$$

\subsubsection{Freundlich Isotherm}

The Freundlich equation [83], in contrast to the Langmuir isotherm, is an empirically derived equation. Here it is assumed that the available sites are heterogeneous and that interactions of adsorbing particles with nearby occupied sites exist. Thermodynamically this can be understood as a logarithmic decrease in the enthalpy of sorption as the surface coverage increases. These assumptions distinguish the Freundlich from the Langmuir isotherm where no interactions are assumed. The Freundlich isotherm is as follows.

$$
q_{e}=K_{F} C_{e}^{1 / n}
$$

where $q_{e}$ is the amount of sorbate taken up, $C_{e}$ is the concentration of sorbate in the solution at equilibrium, $K_{F}$ is Freundlich constant which is related to the maximum loading capacity and $n$ is a parameter related to the intensity of the sorption with $n>$ 1. Even though the equation does not converge to a value when $C_{e} \rightarrow \infty$ it has been reported that it reaches a maximum value if $C_{e}$ is very high. Adamson [84] states that if the equation fit the data it is likely, but not proven, that the surface is heterogeneous.

Equation 3.31 may be rearranged into the linear form shown in eq. 3.32 to ease calculation. Where the value of $\mathrm{n}$ and $\mathrm{K}_{\mathrm{F}}$ may be calculated from the slope and intercept, respectively.

$$
\ln \left(q_{e}\right)=\ln \left(\mathrm{K}_{\mathrm{F}}\right)+\frac{1}{n} \ln \left(C_{e}\right)
$$




\subsubsection{Temkin Isotherm}

The sorption behaviour of many systems do not comply with the Langmuir isotherm [84]. Studies of the temperature dependence over the extent of the surface coverage have revealed a nearly linear dependence in some cases (e.g. $\mathrm{H}_{2}$ onto $\mathrm{Fe}$ or Ni). Since the Langmuir isotherm relies on the assumption that the heat of sorption is independent of the surface coverage this model is not applicable to such cases.

In the derivation of the Temkin isotherm it is assumed that the fall in the heat of sorption is linear rather than logarithmic, as implied in the Freundlich equation. The Temkin isotherm has generally been applied in the following form for the uptake of adsorbates in solution onto solid adsorbents,

$$
q_{e}=\frac{R T}{b_{t}} \ln \left(a_{t} C_{e}\right)
$$

where $R$ is the general gas constant $8.314 \mathrm{~J} \mathrm{~mol}^{-1} \mathrm{~K}^{-1}, T$ is absolute temperature in $\mathrm{K}$, $b_{T}$ and $a_{T}$ represents the Temkin isotherm constants, respectively.

\subsection{Results and discussion on the equilibrium of the sorption of $\mathrm{Cu}^{2+}$}

In order to study the equilibrium of the sorption one must allow enough time to reach the equilibrium. Taking into consideration the kinetic curves shown in Figure 3.3, the uptake of copper reaches equilibrium after 120 minutes have elapsed. Experiments were left 24 hours in order to ensure the arrival at equilibrium point was reached.

Figure 3.29 shows the experimental results for isotherms of $\mathrm{Cu}^{2+}$ uptake at 293, 303, 313, 323 and $333 \mathrm{~K}$. The experiments were carried out as described in Section 2.3.2, procedure A, and were fitted to the Langmuir, Freundlich and Temkin model. The isotherms reflect not only the way molecules are being adsorbed on the surface but also the energy changes when the molecules are adsorbed $\left(\Delta G, \Delta H^{\circ}\right.$ and $\left.\Delta S^{\circ}\right)$. 


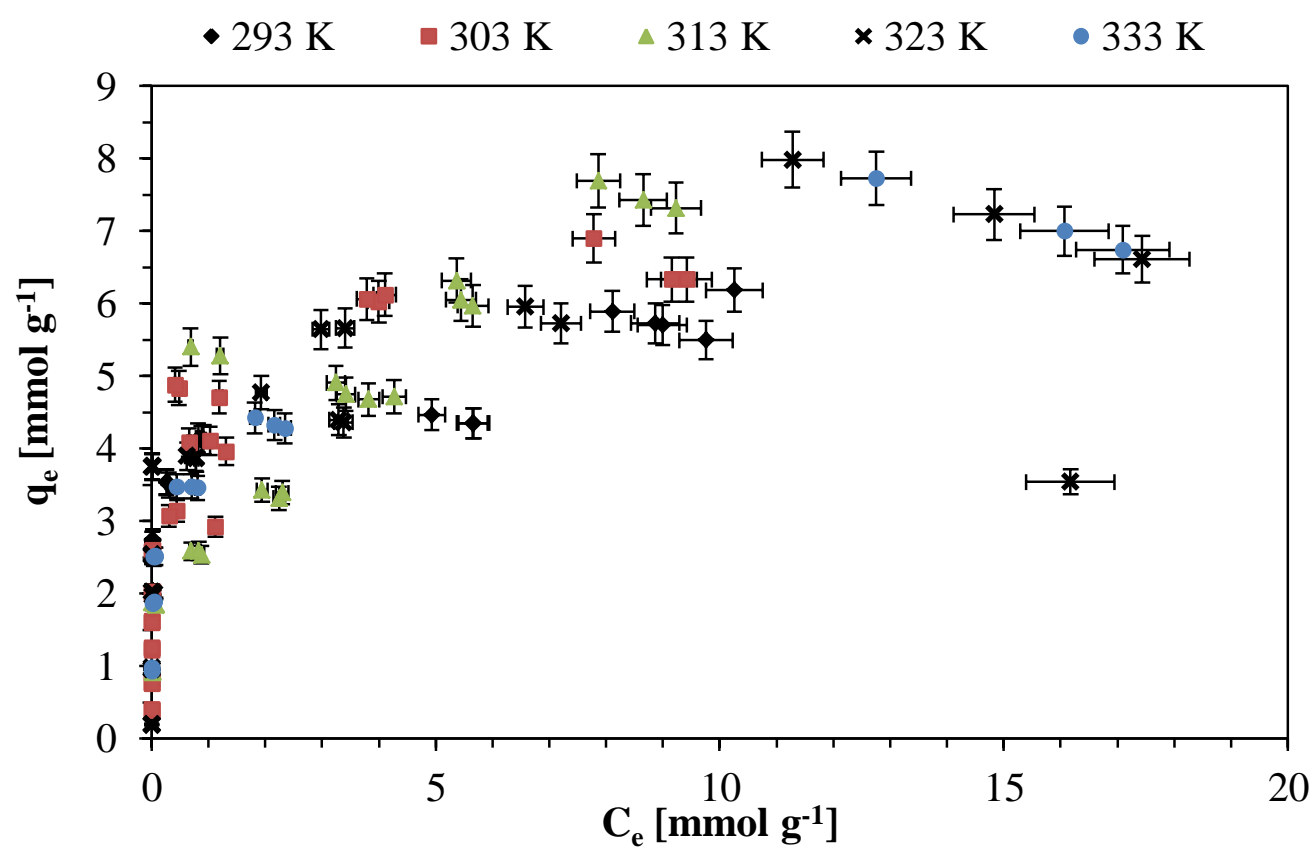

Figure 3.29: $\mathrm{Cu}^{2+}$ adsorption isotherms obtained using procedure $\mathrm{A}$ for temperatures ranging 293 to $333 \mathrm{~K}$. Experimental conditions are described in section 2.3.2.

The data exhibits an irreversible type isotherm which is characterized by having a sharp beginning, also described in environmental and soil chemistry as an $\mathrm{H}$ shaped isotherm [85]. This large rate $\left(\delta q_{\mathrm{e}} / \delta C_{\mathrm{e}}\right)$ is typical for adsorbates with high affinity towards the surface of the adsorbent material. The maximum sorption capacity under these experimental conditions is around $6 \mathrm{mmol} \mathrm{g}^{-1}$, which suggests that not all the binding sites were used compared to previous data were a maximum of $10.4 \mathrm{mmol} \mathrm{g}^{-1}$ is reached. This is directly related to poor stirring leading to an incomplete disaggregation of the particles and high dispersion of the experimental data. Therefore the method had to be changed to a system that offered higher stirring speeds. Nonetheless, these results could be closer to what we can expect during the operation of a packed column with the material in which no stirring is present.

Parameters for the Freundlich, Langmuir, and Temkin models are shown in Table 3.11 . 


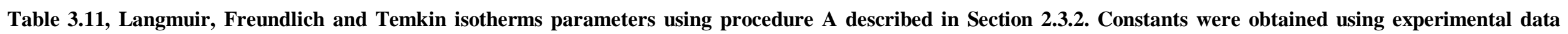
plotted in Figure 3.29.

\begin{tabular}{|c|c|c|c|c|c|c|c|}
\hline $\begin{array}{c}\mathbf{T} \\
{[\mathbf{K}]}\end{array}$ & Model & $\begin{array}{c}q_{m} \\
{\left[\mathbf{m m o l ~ g}^{-1}\right]}\end{array}$ & $\begin{array}{c}K_{F} \\
{\left[\mathrm{~L}^{1 / \mathbf{n}} \mathrm{mmol}^{(\mathrm{n}-1) / \mathbf{n}} \mathrm{g}^{-1}\right]} \\
K_{L} \\
{\left[\mathrm{~L} \mathrm{mmol} \mathbf{~ m m o l}^{-1}\right]} \\
\end{array}$ & $n$ & $\begin{array}{c}a_{T} \\
{\left[\mathbf{L ~ \mathbf { m m o l } ^ { - 1 }}\right]}\end{array}$ & $\begin{array}{c}b_{T} \\
{\left[\mathrm{mmol} \mathrm{mol} \mathrm{g} \mathbf{g}^{-1} \mathbf{J}^{-1}\right]}\end{array}$ & $r^{2}$ \\
\hline & Temkin & & & & 4391 & 4789 & 0.91 \\
\hline \multirow[t]{3}{*}{293} & Freundlich & & 3.99 & 6.74 & & & 0.90 \\
\hline & Langmuir & 4.76 & 72.13 & & & & 0.80 \\
\hline & Temkin & & & & 2603 & 4229 & 0.86 \\
\hline \multirow[t]{3}{*}{303} & Freundlich & & 4.33 & 5.06 & & & 0.88 \\
\hline & Langmuir & 4.98 & 87.87 & & & & 0.76 \\
\hline & Temkin & & & & 168 & 3199 & 0.71 \\
\hline \multirow[t]{3}{*}{313} & Freundlich & & 3.42 & 3.06 & & & 0.81 \\
\hline & Langmuir & 7.45 & 0.77 & & & & 0.70 \\
\hline & Temkin & & & & 8173 & 5228 & 0.75 \\
\hline \multirow[t]{3}{*}{323} & Freundlich & & 4.24 & 6.71 & & & 0.74 \\
\hline & Langmuir & 5.14 & 136.45 & & & & 0.66 \\
\hline & Temkin & & & & 772 & 4161 & 0.90 \\
\hline \multirow[t]{2}{*}{333} & Freundlich & & 3.94 & 4.73 & & & 0.96 \\
\hline & Langmuir & 5.36 & 13.95 & & & & 0.70 \\
\hline
\end{tabular}


Results shown in Table 3.11 exhibit a low $r^{2}$ to the three studied models, except at 293 and $333 \mathrm{~K}$ where a good correlation to Langmuir and Freundlich model may be observed, with $r^{2}$ values higher than 0.9. Nevertheless, no clear trend in the value of the different model constants with a change in the temperature of the reaction may be observed. This could have been due to the poor stirring noted previously.

In order to obtain results with better resolution experiments were repeated using a stirred flask in order to improve disaggregation of the surface enabling new surface to become exposed. Experimental data gathered using procedure B, described in section 2.3.2, are presented in Figure 3.30.

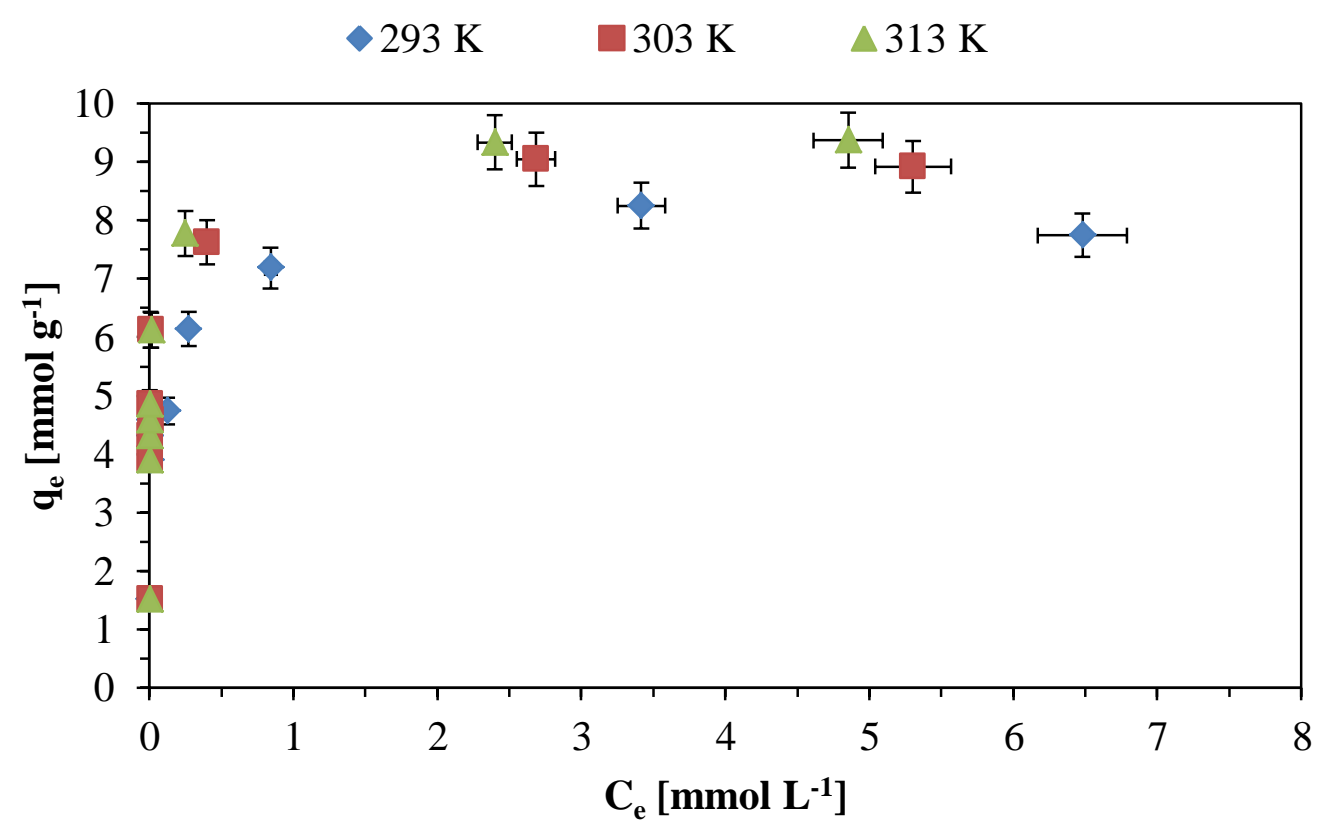

Figure 3.30: Adsorption isotherms obtained using procedure B for 293, 303, $313 \mathrm{~K}$.

Results in Figure 3.30 show less scattering of the experimental data points than those shown in Figure 3.29. A clear trend may be observed as the temperature rises. Furthermore, the maximum adsorption capacity, $q_{m}$, increases by nearly $20 \%(1.5$ mmol g$~^{-1}$ ) with a $20 \mathrm{~K}$ temperature increase in the system. This result shows the importance of studying the thermodynamics of the adsorption to understand the variation that an adsorbent might endure in real-life applications.

The experimental data were fitted to Langmuir, Freundlich and Temkin model and the results of these fits are presented in Table 3.12. The plots for the previously mentioned fits for the Langmuir and Freundlich isotherms are shown in appendix A-1 and A-2, while the Temkin isotherm was fitted using Origin 8.0 fitting tool. 
Table 3.12: Langmuir, Freundlich and Temkin adsorption isotherms parameters using procedure B described in Section 2.3.2. Constants were obtained using experimental data plotted in Figure 3.30.

\begin{tabular}{|c|c|c|c|c|c|c|c|}
\hline $\begin{array}{l}\mathbf{T} \\
{[\mathbf{K}]}\end{array}$ & Model & $\underset{\left[\mathrm{mmol} \mathrm{g}^{-1}\right]}{q_{m}}$ & $\begin{array}{c}K_{F} \\
{\left[\mathrm{~L}^{1 / \mathbf{n}} \mathrm{mmol}^{(\mathrm{n}-1) / \mathrm{n}} \mathrm{g}^{-1}\right]} \\
K_{L} \\
{\left[\mathrm{~L} \mathrm{mmol}^{-1}\right]}\end{array}$ & $n$ & $\begin{array}{c}a_{T} \\
{\left[\mathrm{~L} \mathrm{mmol}^{-1}\right]}\end{array}$ & $\begin{array}{c}b_{T} \\
{[\mathrm{mmol} \mathrm{mol} \mathrm{g}} \\
\mathbf{m o l}^{-1}\end{array}$ & $r^{2}$ \\
\hline & Temkin & & & & 63163 & 3865 & 0.8371 \\
\hline \multirow[t]{3}{*}{293} & Freundlich & & 6.80 & 10.94 & & & 0.8789 \\
\hline & Langmuir & 7.87 & 42.33 & & & & 0.9987 \\
\hline & Temkin & & & & 238905 & 4276 & 0.6900 \\
\hline \multirow[t]{3}{*}{303} & Freundlich & & 8.05 & 10.00 & & & 0.9119 \\
\hline & Langmuir & 8.28 & 61.92 & & & & 0.9998 \\
\hline & Temkin & & & & 174429 & 4211 & 0.6700 \\
\hline \multirow[t]{2}{*}{313} & Freundlich & & 8.39 & 9.04 & & & 0.9058 \\
\hline & Langmuir & 8.34 & 62.53 & & & & 0.9999 \\
\hline
\end{tabular}

*The value of the constants for the Temkin isotherm were obtained using Origin 8.0 fitting tool. 
Results in Table 3.12 shows that experimental data displayed in Figure 3.30 offers a good fit for the Langmuir model with $r^{2}$ values greater than 0.9987 . There is an increase of the value of $K_{L}$ with the temperature, which means affinity of copper towards the surface increases as the temperature raises. Additionally the maximum adsorption capacity $q_{m}$ increases by $0.47 \mathrm{mmol} \mathrm{g}^{-1}$ when the temperature increases from 293 to $313 \mathrm{~K}$.

\subsubsection{Thermodynamical Analysis}

With every chemical reaction, the adsorption of molecules or atoms onto the surface of a solid will undergo energy changes due to changes in the system. During the adsorption process there will be a release or a gain of heat, often referred to as heat of adsorption. The adsorption will be characterized by its spontaneity and the entropy change produced in the system.

Thermodynamic studies in adsorption experiments allow information to be obtained about the stability of the formed product, spontaneity of the process and if there are any changes in the adsorbents loading capacity and equilibrium with temperature change. The thermodynamic values are important not only to compare between sorbents but also because in industrial operations temperature and pressure may vary with the location (altitude) or between day and night. This can lead to a lesser or a greater extent of contaminant removal by the sorbent.

The equilibrium constant of a process can be used to obtain thermodynamical information such as $\Delta H^{\circ}$ (standard enthalpy), $\Delta G$ (Gibbs free energy), $\Delta S^{\circ}$ (standard entropy) giving a better understanding of the process. To do so the van't Hoff equation is used, the derivation of which is as follows [86].

Since,

$$
-\ln K=\frac{\Delta G}{R T}
$$

where $R$ is the gas constant $8.314 \mathrm{~J} \mathrm{~mol}^{-1} \mathrm{~K}^{-1}, T$ is the temperature in $\mathrm{K}, K$ is the equilibrium constant of the process, $\Delta G$ is the standard Gibbs energy.

The variation of $\ln K$ with the temperature is

$$
\frac{d \ln K}{d T}=-\frac{1}{R} \frac{d}{d T}\left(\frac{\Delta G}{T}\right)
$$

In order to develop eq. 3.35 one has to use eq. 3.36 known as the Gibbs-Helmholtz equation 


$$
\left(\frac{d}{d T} \frac{\Delta G}{T}\right)=-\frac{\Delta H^{o}}{T^{2}}
$$

Combining eq. 3.36 with eq. 3.37 van't Hoff's equation is obtained,

$$
\frac{d \ln K}{d T}=\frac{\Delta H^{\circ}}{R T^{2}}
$$

knowing that,

$$
\frac{d T}{T^{2}}=-d\left(\frac{1}{T}\right)
$$

the following form is obtained,

$$
\frac{d \ln K}{d\left(\frac{1}{T}\right)}=\frac{-\Delta H^{\circ}}{R}
$$

Combining the following thermodynamic expressions given by eq. 3.40 and 3.41 ,

$$
\begin{gathered}
\Delta G=\Delta H-T \Delta S \\
-R T \ln K=\Delta G
\end{gathered}
$$

eq. 3.42 is obtained,

$$
\ln K=\frac{\Delta S^{\circ}}{T}-\frac{\Delta H^{\circ}}{R T}
$$

Using the van't Hoff differential equation, $\ln K$ against the inverse of the temperature can be plotted to obtain $\Delta H^{\circ}$ and $\Delta S^{\circ}$. Where, $K$ is the equilibrium constant $\left(K_{L}\right.$, the Langmuir constants can be used), $R$ is gas constant $8.314 \mathrm{~J} \mathrm{~mol}^{-1} \mathrm{~K}^{-1}$ and $T$ is the temperature in $\mathrm{K}$.

This equation gives an explanation of why there is a variation in the adsorption capacity at equilibrium as the temperature changes. For example, if the temperature increases in a system where the adsorption process is endothermic and/or has a positive entropy value this will inevitably displace the equilibrium towards the formation of products.

The modulus of the standard enthalpy can be taken as an indication of whether the process corresponds to a chemical or a physical adsorption. Often small $\Delta H^{\circ}$ values are associated with physical adsorption since the interaction with the surface is weaker, while higher values are expected for chemical sorption since bonds are usually formed. Chemical adsorptions involve the formation of covalent bonds, hydrogen bonds or complexes and similar type of bonds. In the literature physisorption of gases on solids 
are reported to have $\Delta H^{\circ}$ values smaller than $20 \mathrm{~kJ} \mathrm{~mol}^{-1}$, while for chemisorption values of $\Delta H^{\circ}$ range between 100 and $400 \mathrm{~kJ} \mathrm{~mol}^{-1}$. If the system is formed by a liquid phase and a solid phase, values are much lower with $\Delta H^{\circ}$ for chemisorption expected to be $>20 \mathrm{~kJ} \mathrm{~mol}^{-1}$.

Values of $K_{L}$ shown in Table 3.12 were used to plot eq. 3.42 in Figure 3.31.

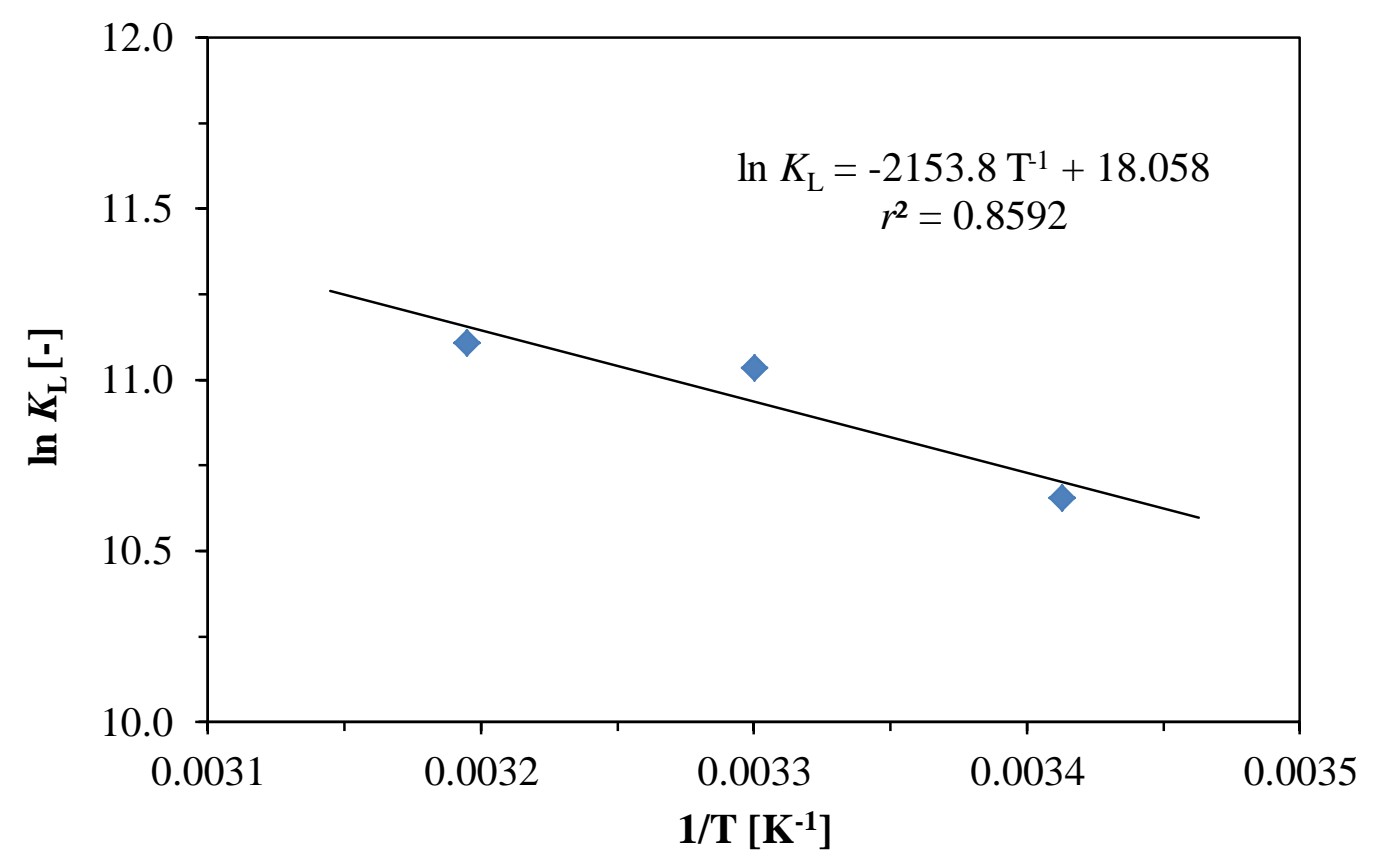

Figure 3.31: Van't Hoff plot using the values of $K_{L}$ shown in Table 3.12 in $\mathrm{L} \mathrm{mol}^{-1}$.

The linear fit exhibits a $r^{2}$ value of 0.8592 which indicates that the gathered data is slightly non linear. Nevertheless, the values of $K_{L}$ are derived from 27 independent experiments shown in Figure 3.30 and A-1, hence the collected data can be considered as reliable.

Values for $\Delta S^{\circ}, \Delta H^{\circ}$ and $\Delta G$ are presented in Table 3.13. These parameters correspond to the overall process as they were obtained from the variation of copper concentration on the surface, thus, they might not reflect the formation of crystals.

Table 3.13: Thermodynamical parameters for the adsorption of $\mathrm{Cu}^{2+}$ onto NCaSil

\begin{tabular}{|c|c|c|c|}
\hline $\begin{array}{c}\mathbf{T} \\
{[\mathbf{K}]}\end{array}$ & $\begin{array}{c}\Delta \mathbf{G} \\
{\left[\mathrm{kJ} \mathrm{mol}^{-1}\right]}\end{array}$ & $\begin{array}{c}\Delta \boldsymbol{H}^{\circ} \\
{\left[\mathrm{kJ} \mathrm{mol}^{-1}\right]}\end{array}$ & $\begin{array}{c}\Delta S^{\circ} \\
{\left[\mathrm{J} \mathrm{mol}^{-1} \mathrm{~K}^{-1}\right]}\end{array}$ \\
\hline 293 & -26.1 & \multirow{4}{*}{17.9} & \multirow{4}{*}{150} \\
\hline 298 & -26.8 & & \\
\hline 303 & -27.6 & & \\
\hline 313 & -29.1 & & \\
\hline
\end{tabular}


The values show that the reaction is spontaneous and endothermic. The increase in the entropy of the system is large, which could be related to the release of $\mathrm{Ca}^{2+}$ and $\mathrm{Si}(\mathrm{OH})_{4}$ into solution. This means that the decrease of entropy due to formation of a crystal structure (wroewolfeite, posnjakite or brochantite) does not account for the positive change of entropy of the dissolution of NCaSil. ${ }^{3}$

Negative entropic changes should arise from:

- $\mathrm{Cu}^{2+}$ being adsorbed onto the surface as $\mathrm{Cu}(\mathrm{OH})_{2}$ or forming crystal (translational)

- Sulfate being removed from solution to form calcium sulfate dihydrate, wroewolfeite, posnjakite and brochantite crystals (translational, rotational and vibrational).

- $\mathrm{OH}^{-}$forming amorphous hydroxides and/or crystal structures (translational, rotational and vibrational)

Positive changes in the entropy should arise from:

- $\mathrm{Ca}^{2+}$ leaching (translational).

- $\mathrm{OH}^{-}$release into solution (translational, rotational and vibrational).

- Hydrolysis of NCaSil structure releasing short chain oligomers and monomeric silica (translational, rotational and vibrational).

$\mathrm{SO}_{4}{ }^{2-}$ and $\mathrm{Si}(\mathrm{OH})_{4}$ are both tetrahedral and $\mathrm{M}_{\mathrm{SO} 4} \approx \mathrm{M}_{\mathrm{SI}(\mathrm{OH}) 4}$ therefore their rotational and translational contributions per mole to the entropy change are similar. Hence the entropy change is going to be to be directly related to the number of moles released and adsorbed of each substance. In the experiment the amount of $\mathrm{SO}_{4}{ }^{2-}$ released ${ }^{4}$ was equal to $2.3 \mathrm{mmol}$, and the amount of $\mathrm{Si}(\mathrm{OH})_{4}$ released into solution was 4 mmol. Implying that the positive entropic contribution of $\mathrm{Si}(\mathrm{OH})_{4}$ is roughly two times larger. In addition the oligomers will also have a contribution to a positive entropy change as stated above.

${ }^{3}$ Electronic contribution were not considered because is small compared to translational, rotational and vibrational.

${ }^{4}$ Amount calculated from Table 3.9 considering that the solid phase is pure brochantite. 
3.4.2 Powder-XRD study on the crystal growth at different temperatures at the equilibrium of the adsorption of copper

The equilibrium of the adsorption of $\mathrm{Cu}^{2+}$ was studied using powder-XRD technique in order to elucidate the dependency of the formation of crystalline phases on the initial concentration and temperature. Results of these experiments are shown from Figure 3.32 to Figure 3.34 .

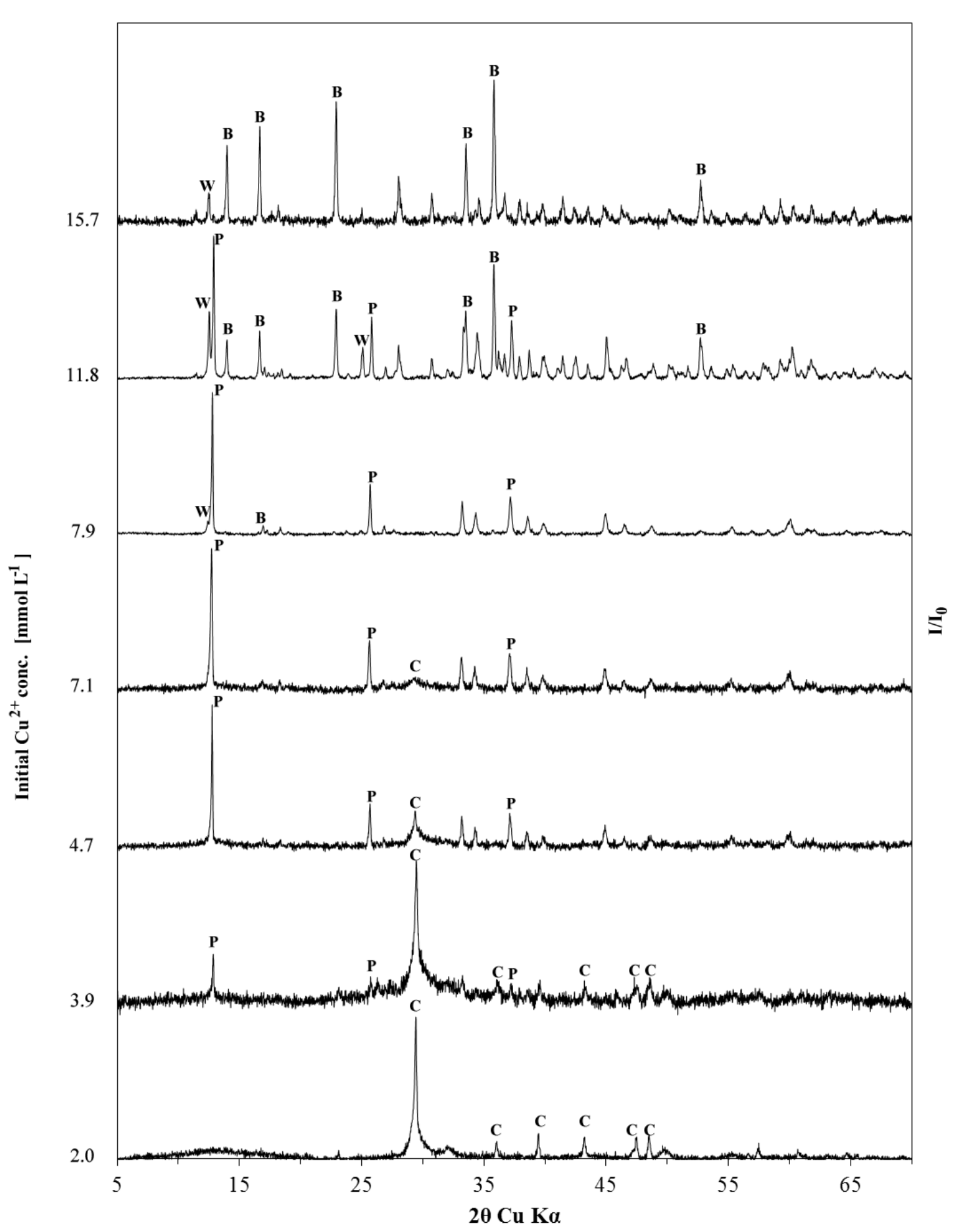

Figure 3.32: Isothermal study at $293 \mathrm{~K}$ at the equilibrium of $\mathrm{Cu}^{2+}$ uptake using $\mathrm{XRD} . \mathrm{C}=$ calcium carbonate, $\mathbf{W}=$ wroewolfeite; $\mathbf{P}=$ posnjakite and $\mathbf{B}=$ brochantite. Experimental conditions: $\left[\mathrm{Cu}^{2+}\right]_{\text {initial }} 15.7 \mathrm{mmol} \mathrm{L}^{-1}$; adsorbent dosage $1 \mathrm{~g} \mathrm{~L}^{-1}$; stirring speed $500 \mathrm{rpm}$. 


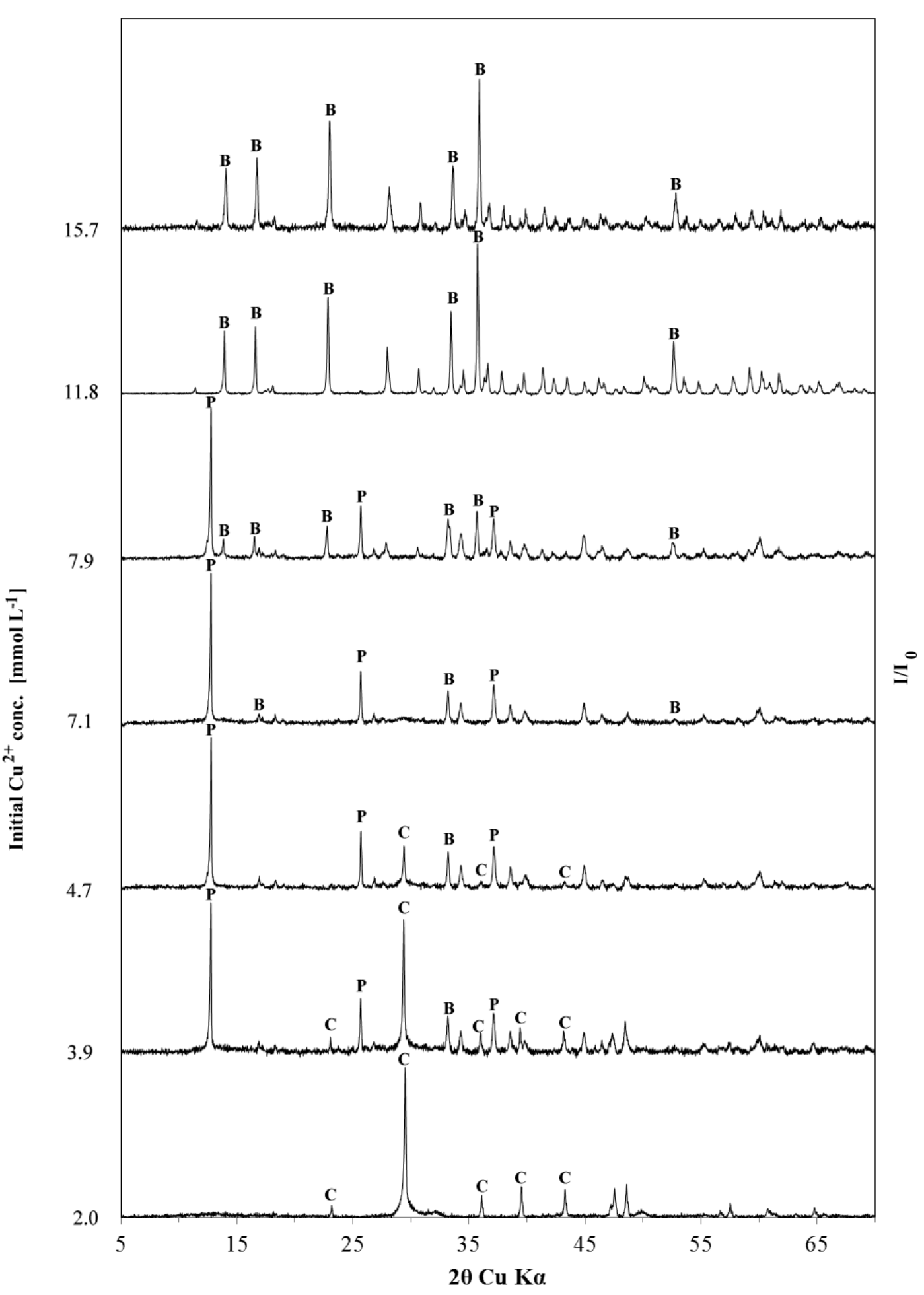

Figure 3.33: Isothermal study at $303 \mathrm{~K}$ at the equilibrium of $\mathrm{Cu}^{2+}$ uptake using $\mathrm{XRD} . \mathrm{C}=$ calcium carbonate, $\mathbf{W}=$ wroewolfeite $\mathbf{P}=$ posnjakite and $\mathbf{B}=$ brochantite. Experimental conditions: $\left[\mathrm{Cu}^{2+}\right]_{\text {initial }} 15.7 \mathrm{mmol} \mathrm{L}^{-1}$; adsorbent dosage $1 \mathrm{~g} \mathrm{~L}^{-1}$; stirring speed $500 \mathrm{rpm}$. 


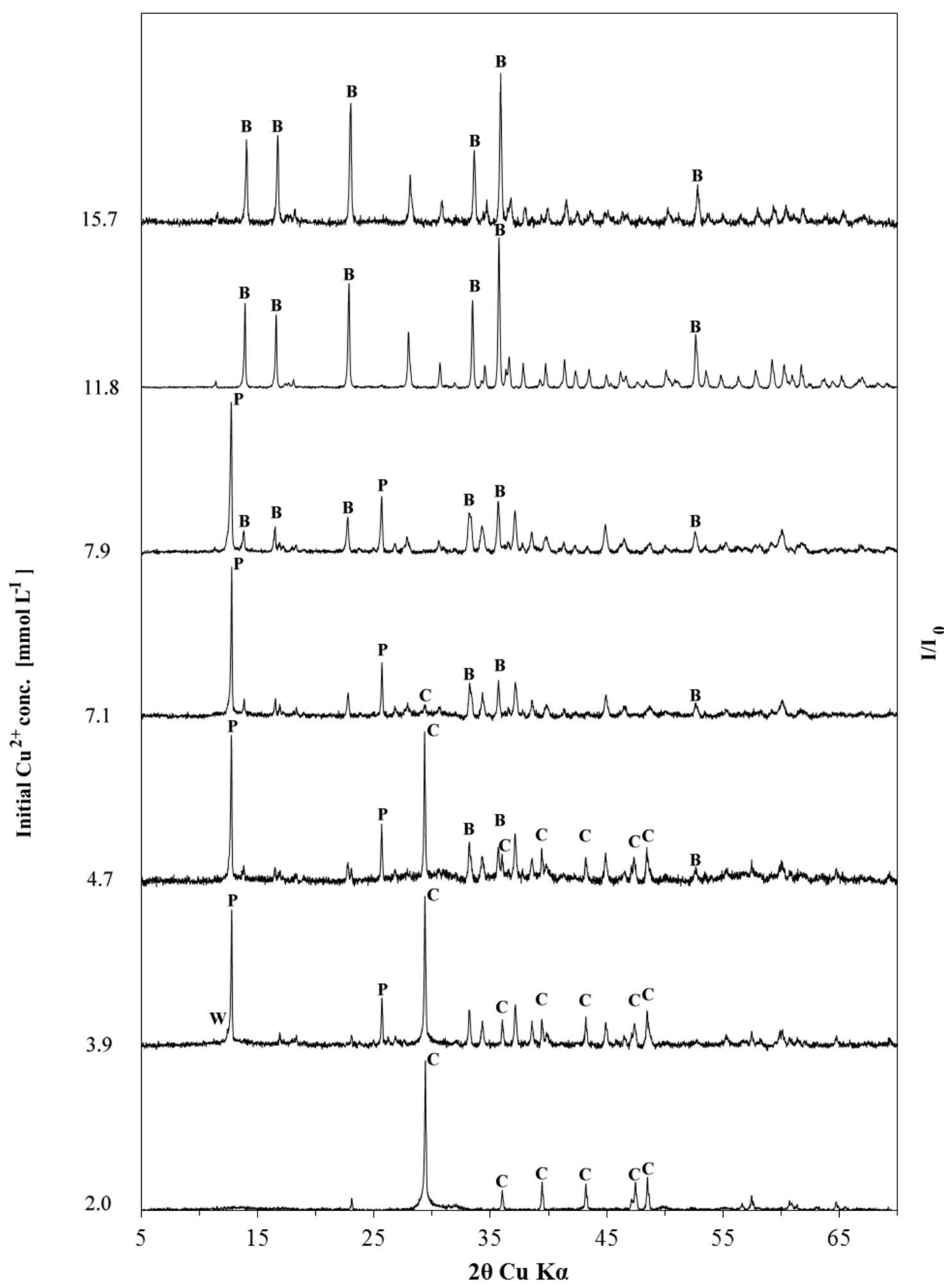

Figure 3.34: Isothermal study at $313 \mathrm{~K}$ at the equilibrium of $\mathrm{Cu}^{2+}$ uptake using XRD. $\mathrm{C}=$ calcium carbonate, $\mathbf{W}=$ wroewolfeite; $P=$ posnjakite and $B=$ brochantite. Experimental conditions: $\left[\mathrm{Cu}^{2+}\right]_{\text {initial }} 15.7 \mathrm{mmol} \mathrm{L}^{-1}$; adsorbent dosage $1 \mathrm{~g} \mathrm{~L}^{-1}$; stirring speed $500 \mathrm{rpm}$.

XRD patterns obtained from the solid portion of the isotherm reveal interesting information. At low initial concentrations of copper the resulting compound on the solid surface is calcium carbonate (C). These peaks do not correspond to calcium sulfate 
dihydrate or NCaSil as this sample reached equilibrium and as it was shown in kinetic studies these compounds form and disappear rapidly. Furthermore, calcium carbonate and gypsum have $K_{s p}$ value of $3.8 \times 10^{-9}$ and $2.4 \times 10^{-5}$, respectively. Hence, the formation of calcium carbonate will be preferred at the equilibrium. The studied system offers the perfect environment for the formation of this compound as the $\mathrm{pH}$ is above 8 at low initial concentration of copper. Calcium and carbon dioxide come from NCaSil and atmospheric $\mathrm{CO}_{2}$ dissolved in solution, respectively.

Moreover, at initial concentrations of copper ions between 3.9 and $7.9 \mathrm{mmol} \mathrm{L}^{-1}$ the formation of posnjakite is favoured at all studied temperatures as it is shown in Figure 3.32, Figure 3.33 and Figure 3.34.

At concentrations higher than $11.8 \mathrm{mmol} \mathrm{L}^{-1}$ the thermodynamic stable crystal structure is brochantite at all studied temperatures. This is consistent with the findings in the kinetic experiments where after $24 \mathrm{~h}$ only brochantite peaks were found.

\subsubsection{Study of the release of calcium at the equilibrium}

One of the important questions that had to be answered was if the sorption of $\mathrm{Cu}^{2+}$ onto $\mathrm{NCaSil}$ involved an ion exchange process between $\mathrm{Ca}^{2+}$ and $\mathrm{Cu}^{2+}$. This concept was supported by Cairns and Borrmann $[38,40]$ since they witnessed the release of $\mathrm{Ca}^{2+}$ over time as copper was adsorbed. They also observed that $\mathrm{Ca}^{2+}$ was released into the water at $\mathrm{pH} 7$ without the need of adding an exchangeable ion such as $\mathrm{Cu}^{2+}$. Hence it was obvious to ask to what extent the release of calcium ions corresponded to an ion exchange process when $\mathrm{Cu}^{2+}$ is added into the solution.

The experimental data gathered from copper isotherms shown in Figure 3.30 were plotted against the concentration of calcium ions in solution at the equilibrium. Figure 3.35 depicts the relationship of the uptake of copper ions and calcium ions at three different temperatures. 


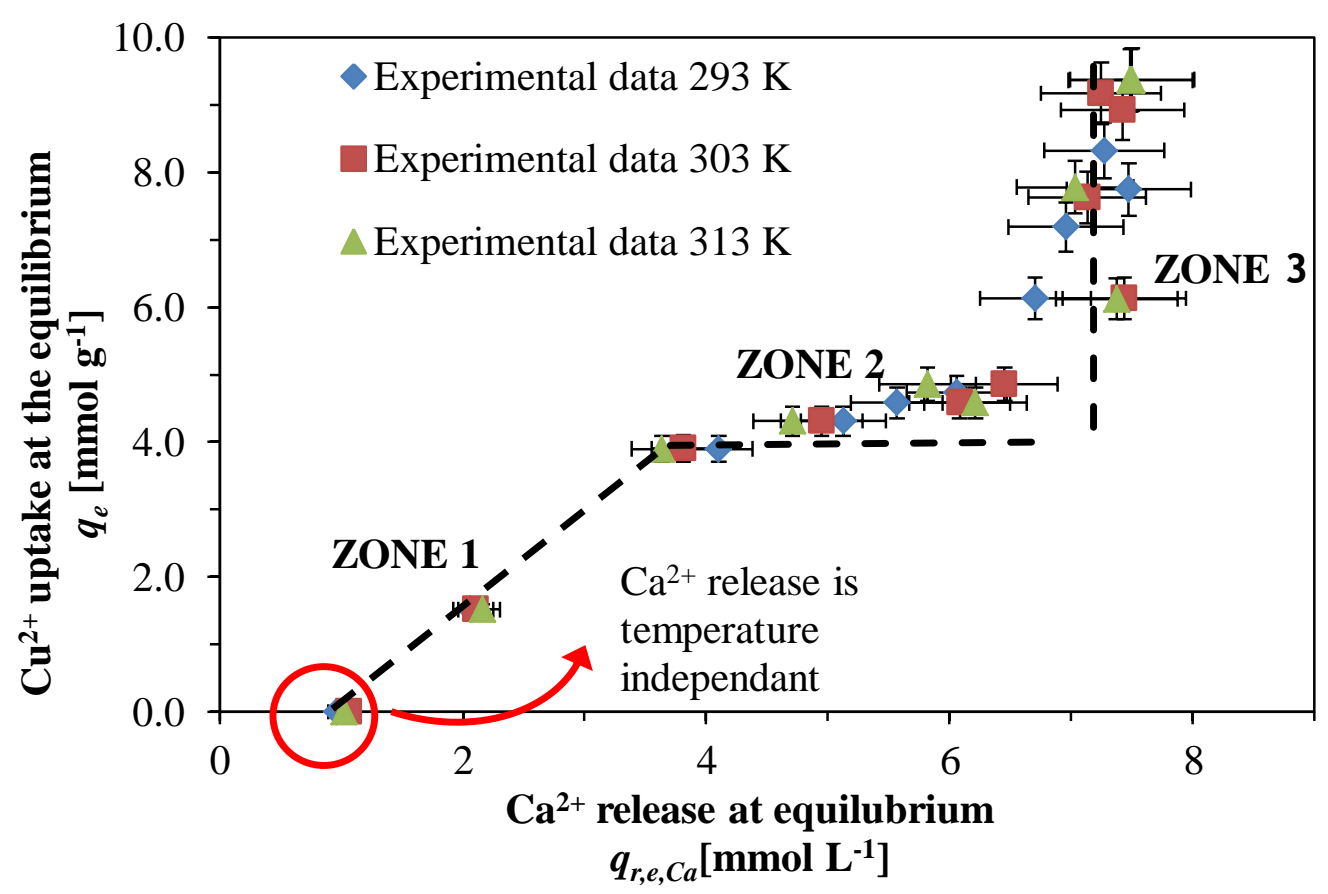

Figure 3.35: Dependency between $\mathrm{Cu}^{2+}$ uptake and $\mathrm{Ca}^{2+}$ release at the equilibrium. Experimental data was gathered as described in procedure $B$ in section 2.3.2.

The data shown in Figure 3.35 may be separated into three different zones: zone 1, corresponds to a linear dependency between $\mathrm{Cu}^{2+}$ being adsorbed onto the surface and calcium ions being released from the surface: zone 2, corresponds to the range where the amount of calcium release is much greater than the amount of $\mathrm{Cu}^{2+}$ being adsorbed; and Zone 3, corresponds to non-dependency of $\mathrm{Cu}^{2+}$ ions being adsorbed to the amount of calcium released. It should also be noted that the amount of calcium released at equilibrium is almost independent of the temperature as the data points for the different temperatures are overlapping in most cases. One could assume that calcium ions are weakly bonded to the surface since the O-Ca bond is easily hydrolysed, causing the release of calcium into solution, even without copper being present in solution. This could suggest an ionic bond rather than a covalent bond.

Studies of the structure of NCaSil suggest that calcium should be found on the surface and holding layers of silicate together as shown in Figure 1.2. However, no difference could be observed in these experiments or those carried out in section 3.2.3 that suggest that certain calcium ions form a stronger bond to $\mathrm{NCaSil}$ or require longer time to leach into solution. 


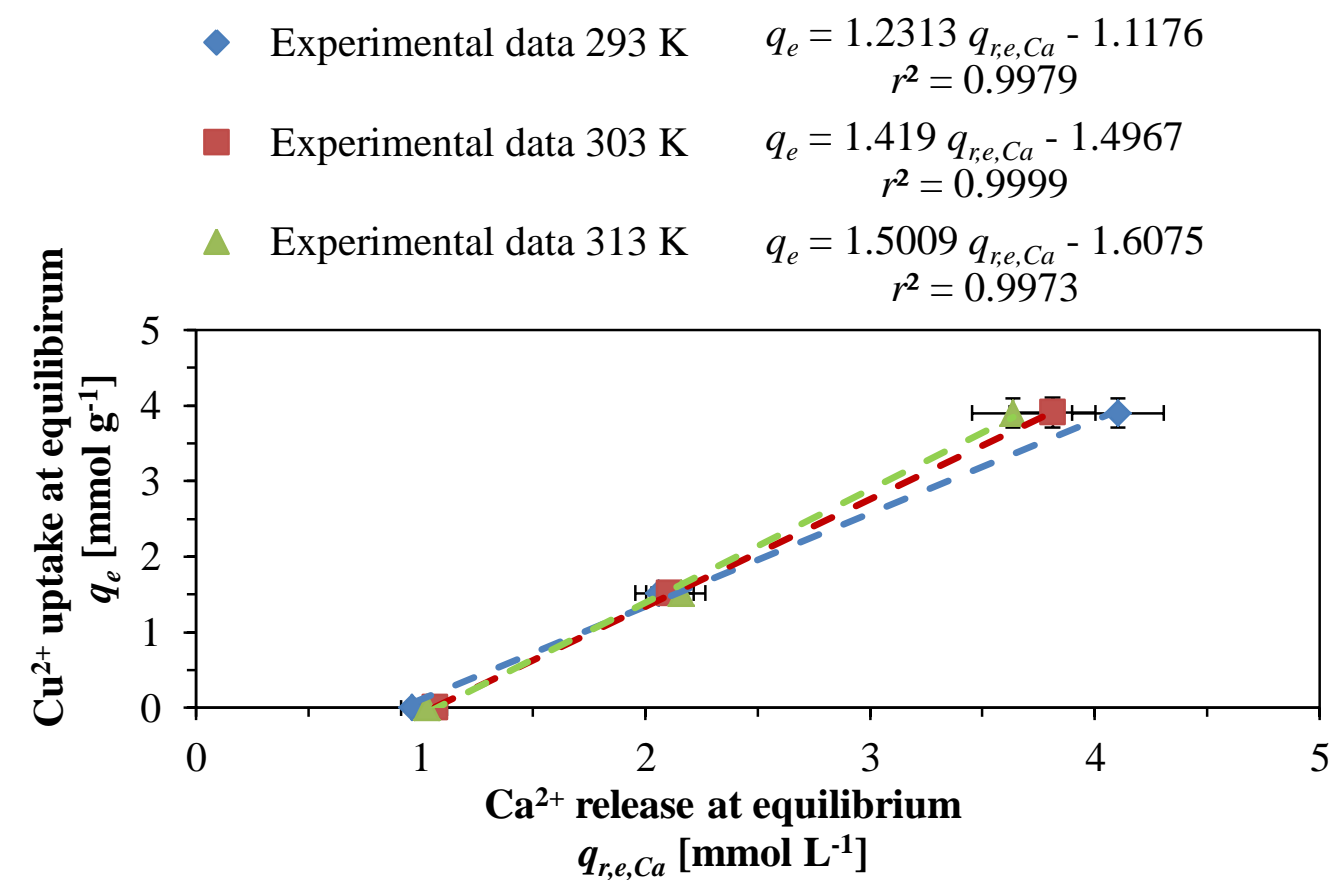

Figure 3.36: Expansion of zone 1 of Figure 3.35. Experimental data was gathered as described in procedure $B$ in section 2.3.2.

Figure 3.36 shows a magnification over zone 1 . The slope of the plot in this region increases slightly as the temperature increases suggesting an endothermic process between copper ion uptake and calcium ion release. The slope for the different data sets varies from 1.2 to 1.5 suggesting the existence of an ion exchange process with a reaction order of 1 to 1.5. Meaning that for every ion of copper being adsorbed there is one ion of calcium being released. The excess could be attributed to $\mathrm{O}-\mathrm{Ca}$ being hydrolysed by the aqueous media. 


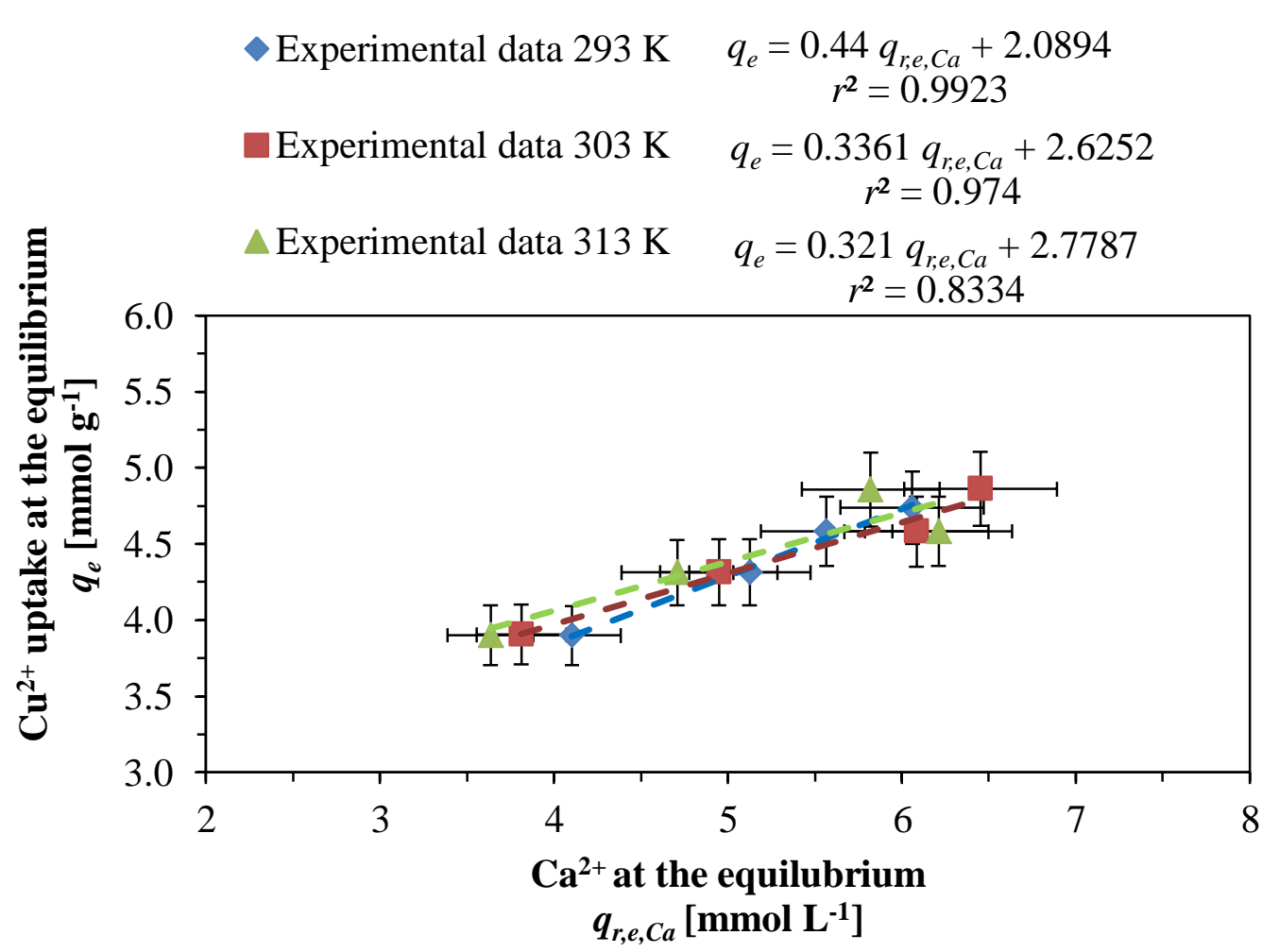

Figure 3.37: Expansion of zone 2 of Figure 3.35. Experimental data was gathered as described in procedure $B$ in section 2.3.2.

Figure 3.37 shows a closer look at zone 2 and linear regressions for the different data sets with slope values varying from 0.321 to 0.44 . Although more copper is adsorbed as the concentration and temperature increases the amount of calcium released in zone 2 is much greater than in zone 1 causing a significant decrease in the value of the slope. For every 10 moles of calcium ions released at the equilibrium 3 to 4 moles of copper ions are adsorbed. This boost on the release of $\mathrm{Ca}^{2+}$ may be explained as the result of a decrease on the initial $\mathrm{pH}$ of the solution due to a higher concentration of copper. 


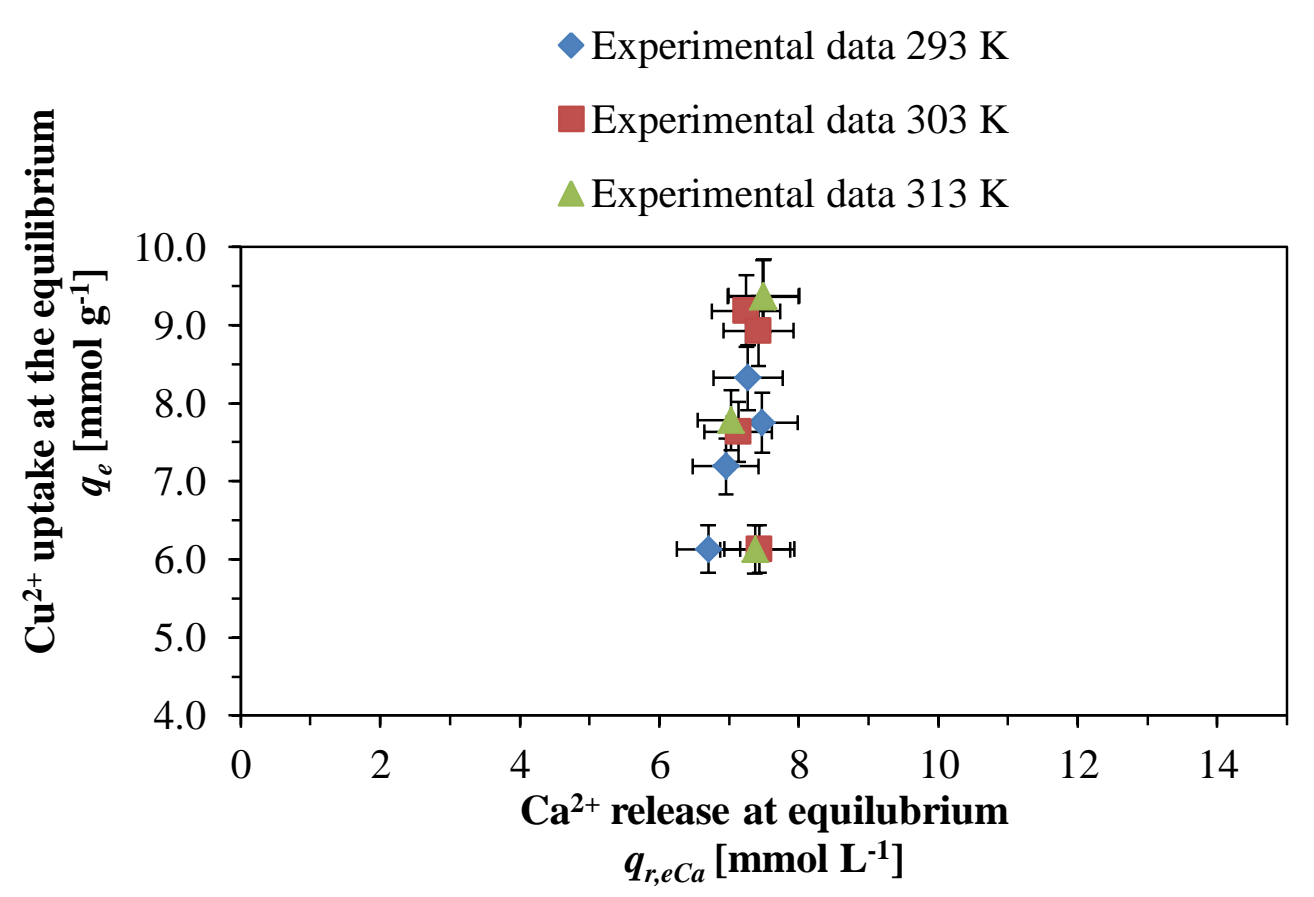

Figure 3.38: Expansion of zone 3 of Figure 3.35. Experimental data was gathered as described in procedure $B$ in section 2.3.2.

In Figure 3.38 it can be observed that copper uptake is independent of calcium release for all studied temperatures. Copper keeps being adsorbed disregarding the fact that no more calcium is released. This result is important since it proves that $\mathrm{Cu}^{2+}$ uptake is not purely dependant on calcium availability. Furthermore, this region also shows the depletion of calcium ions in NCaSil, with a value near $7 \mathrm{mmol}$ of $\mathrm{Ca}^{2+}$ per gram of NCaSil (refer to Eq. 3.25 in section 3.2.6).

\subsubsection{Study of the release of monomeric silica at the equilibrium}

After discovering the complex relation between $\mathrm{Ca}^{2+}$ and $\mathrm{Cu}^{2+}$ during the uptake mechanism, a second question had to be answered. To what extent is the release of monomeric silica related to the uptake of copper ions? The amount of $\mathrm{Cu}^{2+}$ adsorbed at the equilibrium obtained from the experimental data gathered from the isotherms in Figure 3.30 against the amount of silicate released at the equilibrium was plotted in Figure 3.39. The plot helps to understand the relation between these two analytes. 


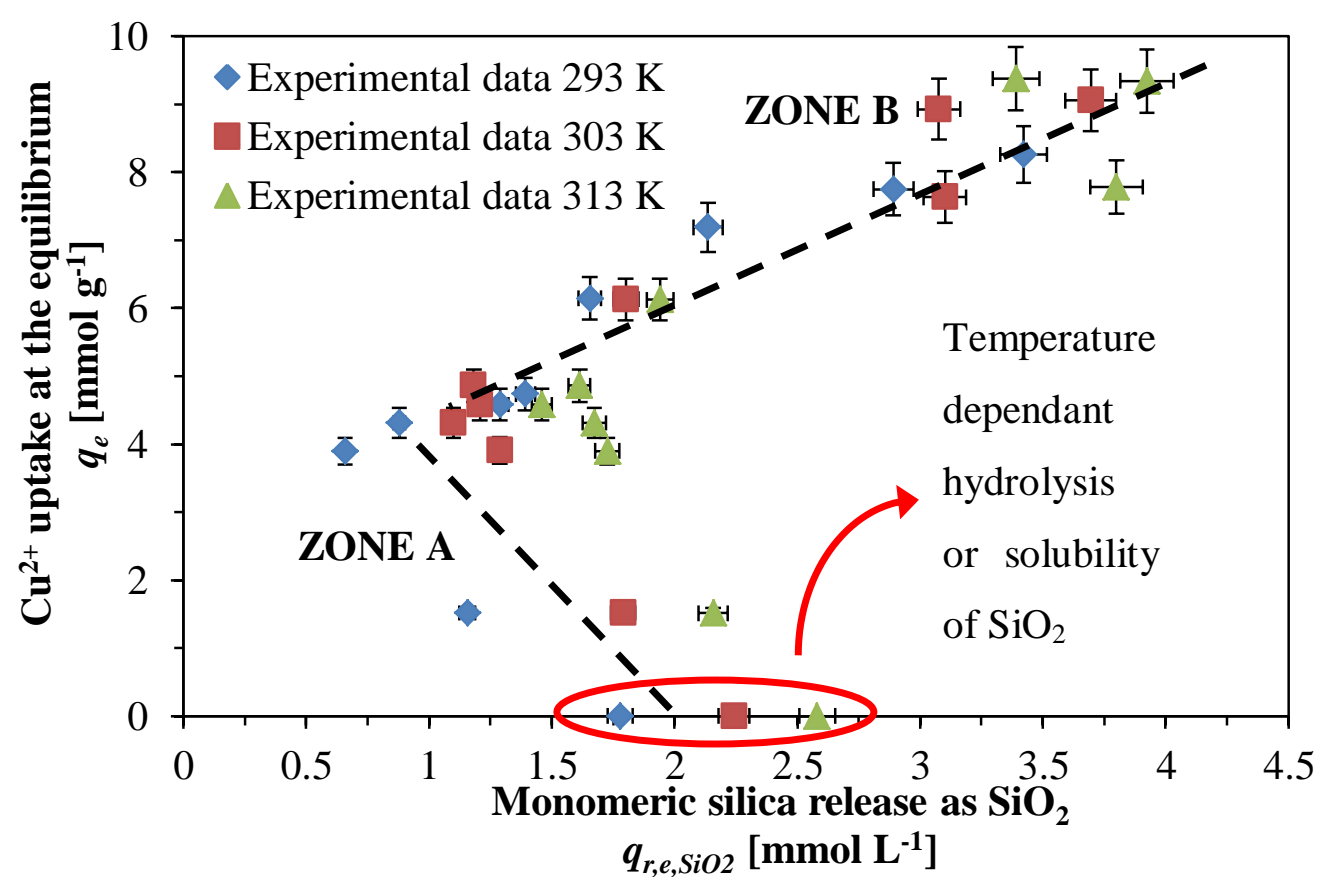

Figure 3.39: Dependency between $\mathrm{Cu}^{2+}$ uptake and monomeric silica release.

Two different zones may be distinguished in Figure 3.39. Zone A represents the range where the amount of silicate released decreases as the amount of $\mathrm{Cu}^{2+}$ absorbed increases and zone $\mathrm{B}$, is the region where the uptake of copper at equilibrium is proportional to the release of silicate at the equilibrium.

The peculiar relation in zone A between both species may be attributed to the passivation of the NCaSil surface due to amorphous copper hydroxide depositing, which does not allow further chemical reactions underneath this layer. Although this cannot be proved to a full extent experimentally, EDS mapping in Figure 3.24 shows that even when no crystals of posnjakite or brochantite are observed in certain portions of the NCaSil copper is detected covering the surface.

Another reason is that the $\mathrm{pH}$ might be in a region where $\mathrm{Si}-\mathrm{O}-\mathrm{Si}$ bonds are comparatively stable. Taking into consideration $\mathrm{pH}$ values at the equilibrium in Table 3.5 and the solubility of amorphous silica at different $\mathrm{pH}$ values shown in Figure 3.7 and in appendix A-3 it is possible to state the trend in Zone A is most likely due to the $\mathrm{pH}$ value of the solution at the equilibrium.

Solubility of amorphous silica is temperature dependant contrasting the trends observed for calcium. This could indicate that the amount of energy required to break $\mathrm{O}-\mathrm{Ca}$ bond is negligible compared to the amount required to hydrolyse the O-Si bond. The O-Ca bond should be weak in the range of ionic bond and $\mathrm{O}-\mathrm{Si}$ is stronger in the range of 
covalent bonds. Furthermore, the temperature dependence of the release of monomeric silica is in good agreement with its solubility as previously shown in Figure 3.7.

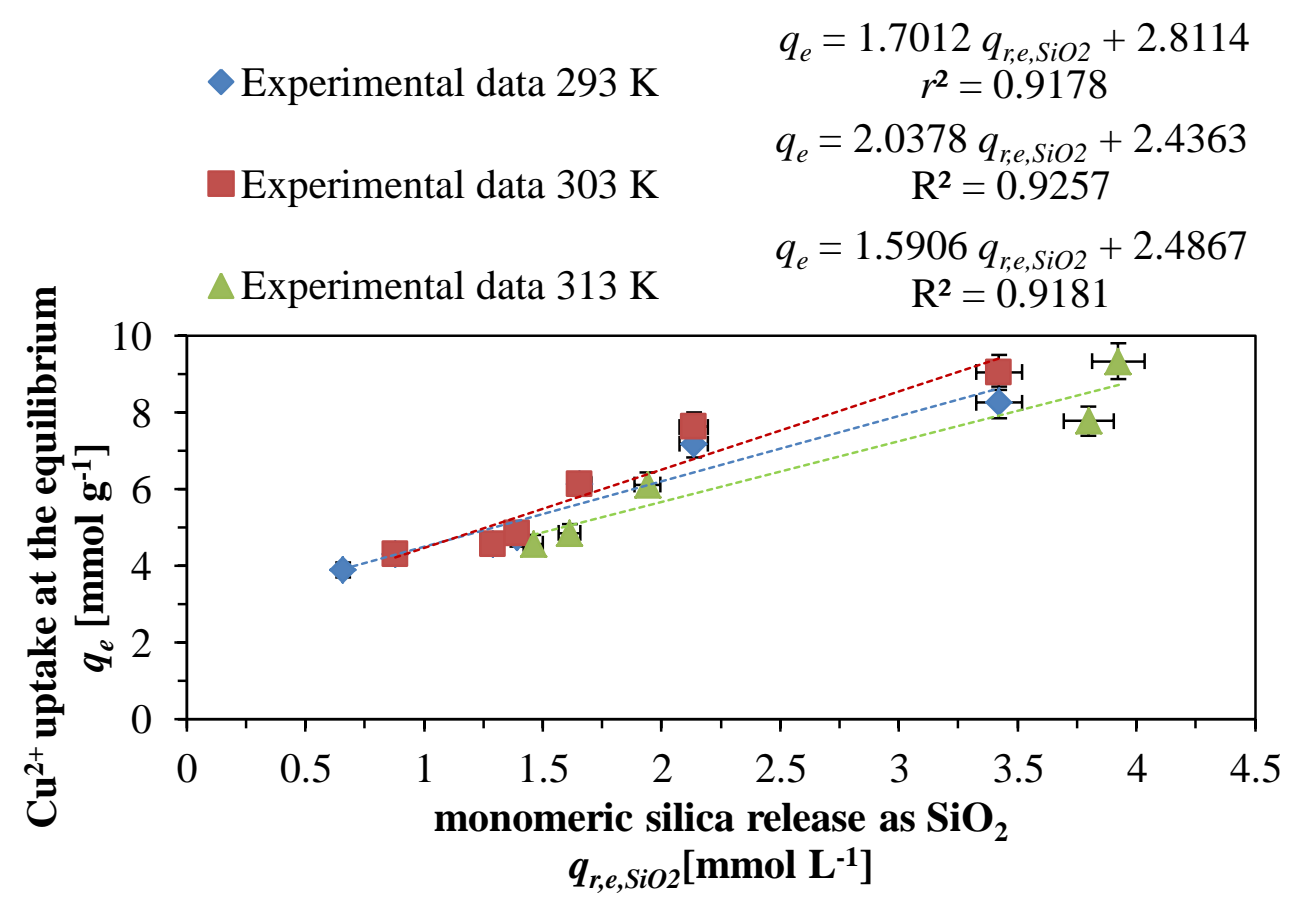

Figure 3.40: Linear regression to Zone B shown in Figure 3.39.

In Figure 3.40 a closer look to zone B is shown including the linear regression for the three data sets. The value of the slopes for the three data sets ranges from 1.6 to 2.0. This means that for every 2 moles of copper being adsorbed 1 mole of silicate is released. The release of silicate will reach a limit which will be related to the solubility at a given temperature of amorphous silica, $\mathrm{pH}$ and adsorbent dosage.

\subsubsection{The effect of the initial $\mathrm{pH}$ of the solution on the amount of copper adsorbed} and the release of $\mathrm{Ca}^{2+}$ and monomeric silica at the equilibrium.

The adsorption of copper is largely driven by the availability of $\mathrm{OH}^{-}$on the surface of NCaSil. Therefore, it is important to study the adsorption of copper varying the $\mathrm{pH}$ of the initial copper solution below its original value around 4.8. This will show the lowest $\mathrm{pH}$ at which $\mathrm{Cu}^{2+}$ may be absorbed from solution using an adsorbent dosage of $1 \mathrm{~g} \mathrm{~L}^{-1}$. Also information regarding the release of $\mathrm{Si}(\mathrm{OH})_{4}$ and $\mathrm{Ca}^{2+}$ may show interesting results which may or may not add further to the discussion of how $\mathrm{Cu}^{2+}$ is adsorbed.

The uptake of $\mathrm{Cu}^{2+}$ at the equilibrium was studied at $\mathrm{pH}$ values of 2,3 and 4 . Results of this experiment are shown in Figure 3.41. 


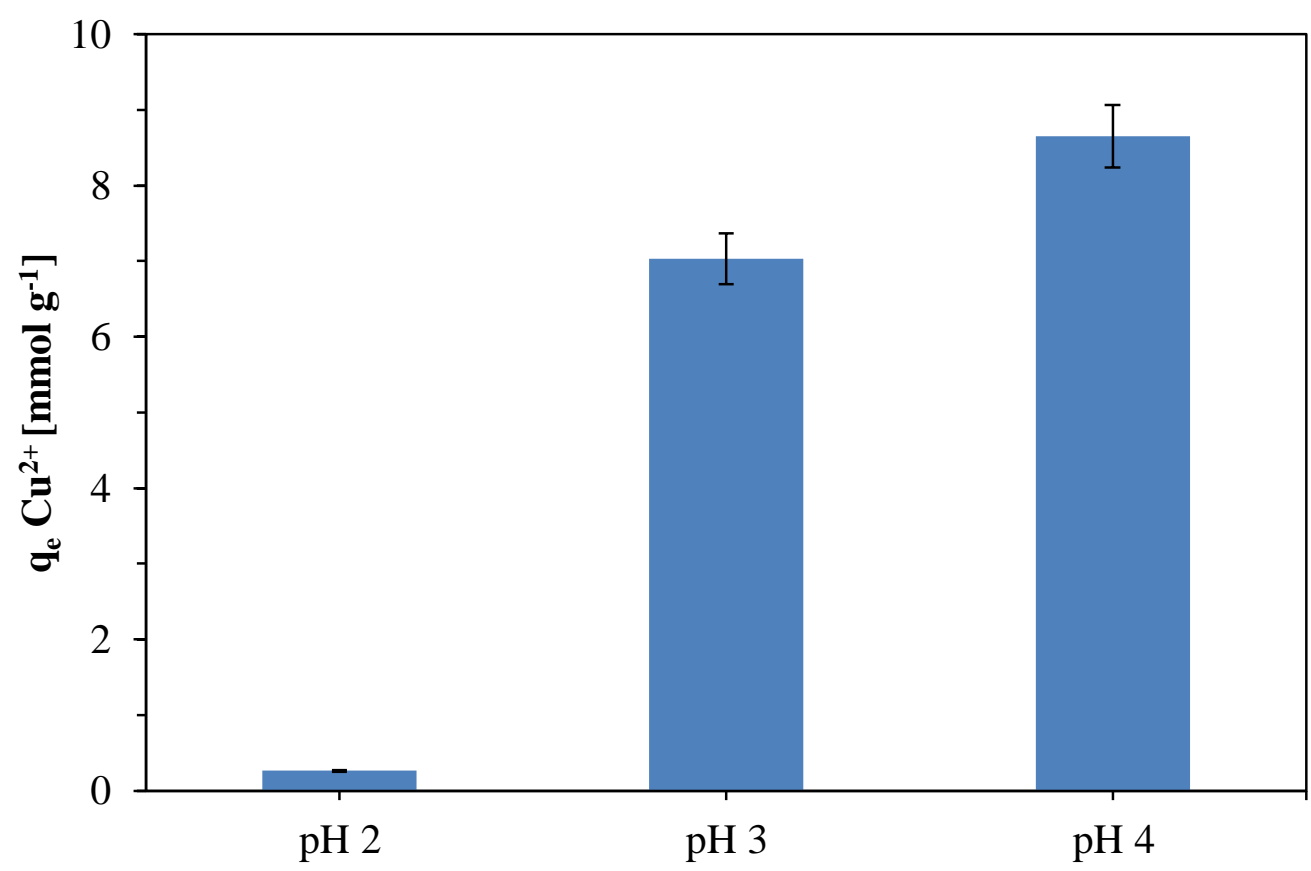

Figure 3.41: $\mathrm{Cu}^{2+}$ adsorption and its relation to the initial $\mathrm{pH}$ of the solution. Experimental conditions: $\left[\mathrm{Cu}^{2+}\right]_{\text {initial }} 15.7 \mathrm{mmol} \mathrm{L}^{-1}$; adsorbent dosage $1 \mathrm{~g} \mathrm{~L}^{-1}$; $\mathrm{pH}$ adjusted with $\mathrm{H}_{2} \mathrm{SO}_{4}$.

It is clear that at least a $\mathrm{pH}$ value of 3 is needed in order to be able to remove copper from solution. This result is of extreme importance since in the following chapter an emulated mining waste which contains a $\left[\mathrm{H}^{+}\right] \approx 1 \mathrm{M}$ will be treated with NCaSil in order to recover copper ions from it. Therefore a stage where the $\mathrm{pH}$ value is modified to 3 is going to be required.

Moreover, the study of the release of calcium and silicate to solution was carried out using different initial $\mathrm{pH}$ values ranging from 2 to 5 without the presence of copper. Results are shown in Figure 3.42. 


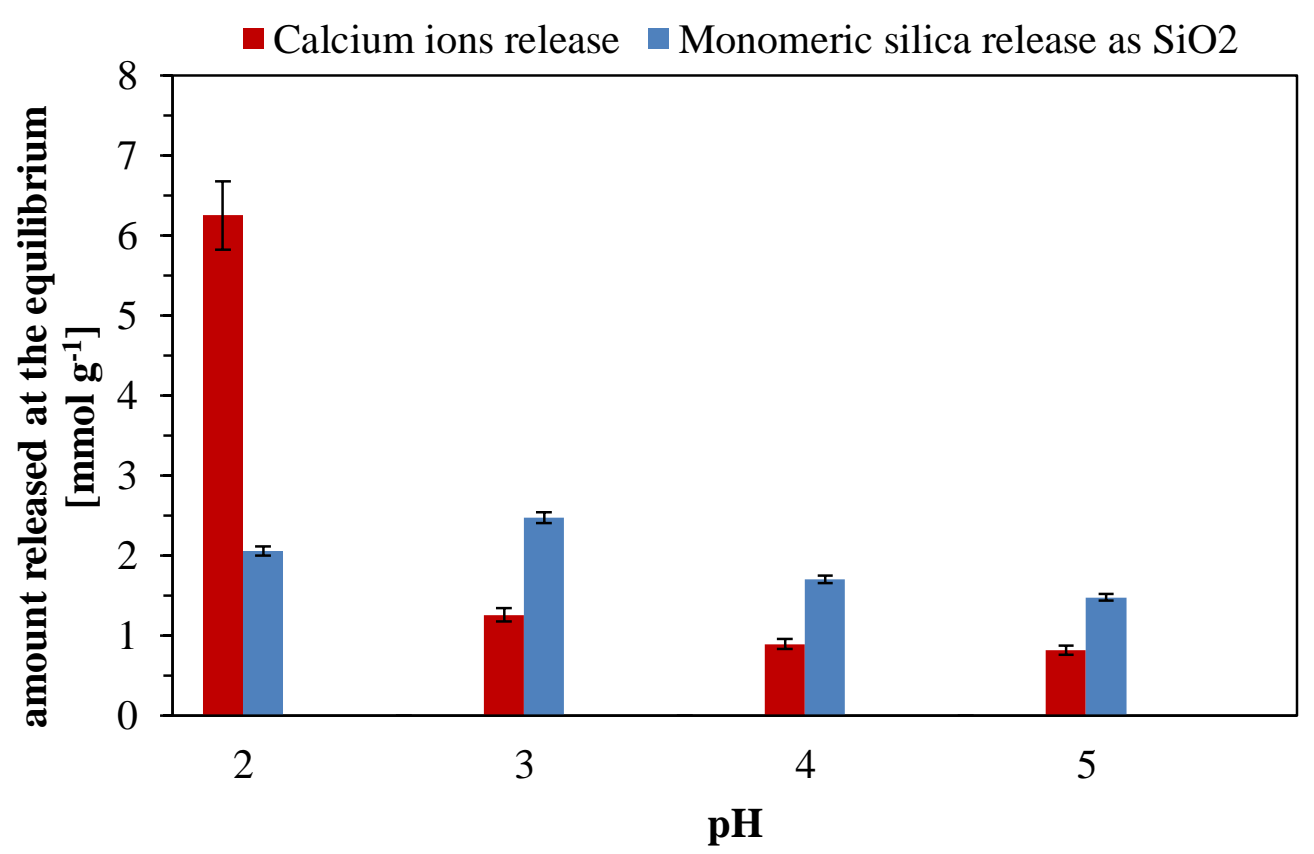

Figure 3.42: $\mathrm{Ca}^{2+}$ and monomeric silica release as a function of $\mathrm{pH}$. Experimental conditions: $\left[\mathrm{Cu}^{2+}\right]_{\text {initial }} 0 \mathrm{mmol} \mathrm{L}{ }^{-1}$; adsorbent dosage $1 \mathrm{~g} \mathrm{~L}^{-1} ; \mathrm{pH}$ adjusted with $\mathrm{H}_{2} \mathrm{SO}_{4}$.

Results show that most of calcium is released at $\mathrm{pH}$ 2. Calcium leaching decreases significantly from a value near 6.1 to $1.5 \mathrm{mmol} \mathrm{g}^{-1} \mathrm{~L}^{-1}$ when the $\mathrm{pH}$ of the solution has a value of 3 . Silicate release shows a maximum leaching at $\mathrm{pH} 3$ which is consistent with the literature where it exhibits a slight increase in solubility at that $\mathrm{pH}$, as shown in appendix to A-3.

The same experiment was carried out in the presence of a $15.7 \mathrm{mmol} \mathrm{L}^{-1}$ copper solution and the results are plotted in Figure 3.43. 


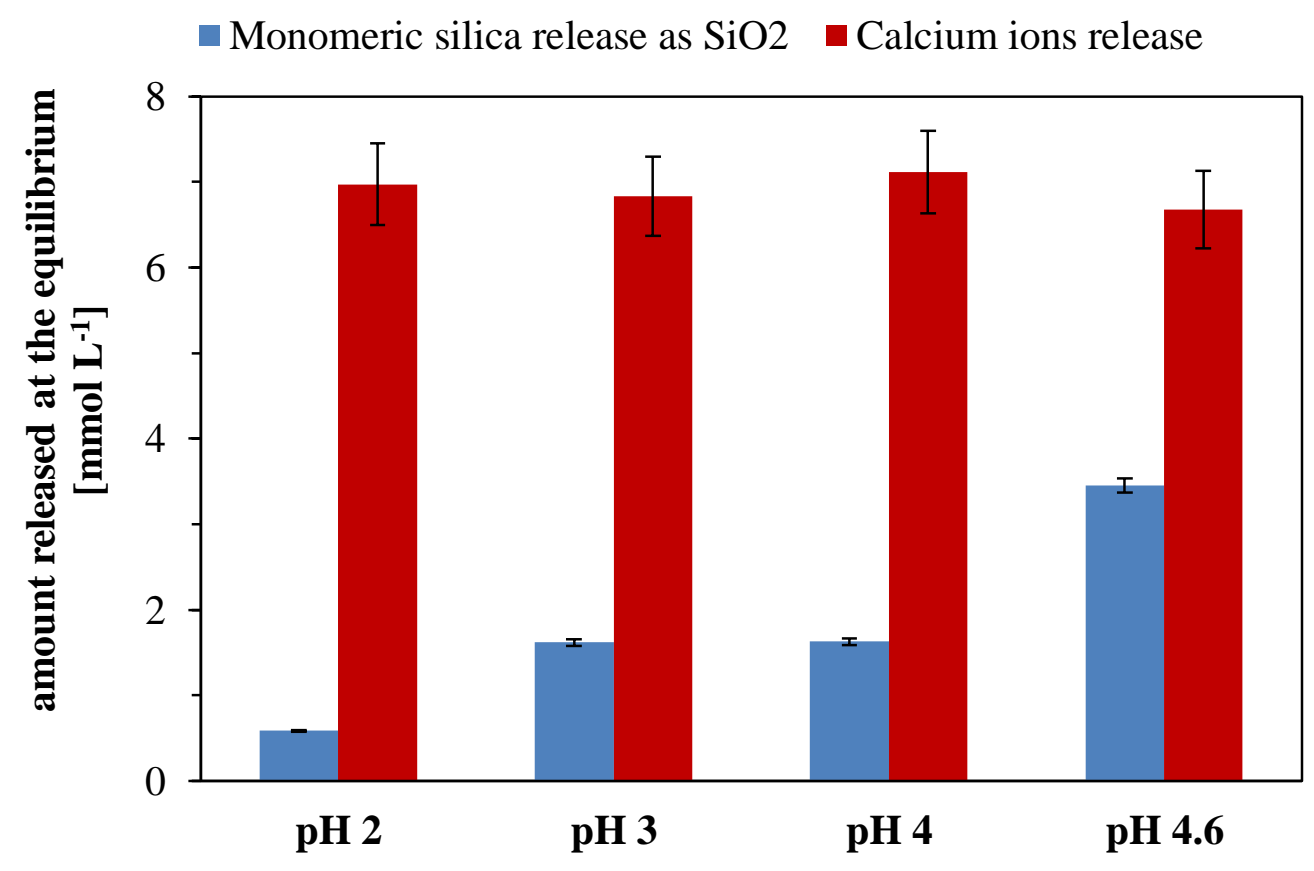

Figure 3.43: $\mathrm{Ca}^{2+}$ and monomeric silica release in the presence of copper at different $\mathrm{pH}$ values. Experimental conditions: $\left[\mathrm{Cu}^{2+}\right]_{\text {initial }} 15.7 \mathrm{mmol} \mathrm{L}^{-1}$; adsorbent dosage $1 \mathrm{~g} \mathrm{~L}^{-1}$; $\mathrm{pH}$ adjusted with $\mathrm{H}_{2} \mathrm{SO}_{4}$.

Calcium is leached out readily on the studied $\mathrm{pH}$ values. The difference between $\mathrm{Ca}^{2+}$ concentrations at the equilibrium lie within the error of the method, so it is not possible to say there is a difference between them. In contrast the release of monomeric silica increases as the $\mathrm{pH}$ value increases. Comparing these results to those obtained in Figure 3.42 it is possible to observe that the presence of $\mathrm{Cu}^{2+}$ ions increases the amount of monomeric silica in solution by $2 \mathrm{mmol} \mathrm{L}^{-1}$ when the initial $\mathrm{pH}$ has a value close to 5 . This could be an indication that $\mathrm{Cu}^{2+}$ is reacting with the silanol groups of the NCaSil and in doing so, promotes the release of silica to solution. 


\subsection{Chapter Conclusions}

$\mathrm{Cu}^{2+}$ adsorption onto NCaSil occurs in a stepwise mechanism that involves physical and chemical processes. The first step involves the disaggregation of NCaSil agglomerates into smaller aggregates exposing a larger surface to the interphase. This step proved to be crucial to allow the maximum adsorption capacity since the capacity doubled when the reaction was vigorously stirred, therefore stirring must be employed.

$\mathrm{Cu}^{2+}$ is then adsorbed onto the surface rapidly fitting exceptionally well to a pseudo-second order equation. The rate increases two orders of magnitude when the temperature increases from 293 to $347 \mathrm{~K}$. The activation energy of the copper adsorption onto NCaSil is $54 \mathrm{~kJ} \mathrm{~mol}^{-1}$, thus copper binds chemically to the surface forming amorphous copper hydroxide that evolves to a crystal structure via a thermodynamical promoted rearrangement of ions on the surface.

The kinetic study with powder-XRD using $15.7 \mathrm{mmol} \mathrm{L}^{-1} \mathrm{Cu}^{2+}$ solution and $1 \mathrm{~g} \mathrm{~L}^{-1}$ of adsorbent dosage showed that the reaction mechanism had intermediates. At $293 \mathrm{~K}$ wroewolfeite and posnjakite were formed for a short period of time, which then transformed into brochantite as shown by eq. 3.18. At $313 \mathrm{~K}$ wroewolfeite could no longer be observed and posnjakite developed rapidly at an early stage of the reaction. After $120 \mathrm{~min}$ brochantite was the only phase present in the solid portion of the reaction. At $333 \mathrm{~K}$, small peaks of posnjakite appear for a very short period of time which then transformed via dehydration to brochantite becoming the main crystal structure after only 30 minutes. Furthermore, the activation energy for the formation of plane 420 of brochantite was calculated to be $44 \mathrm{~kJ} \mathrm{~mol}^{-1}$.

The reaction is complex as it involves other species besides copper sulfate and NCaSil. A simplified reaction schematic is depicted in Figure 3.44. At the same time that copper was being adsorbed, calcium, silicate and hydroxyl ions were released into solution. This leaching of NCaSil constituents caused the irreversible loss of structure, hence once contacted with an aqueous solution the material cannot be regenerated and reused. 


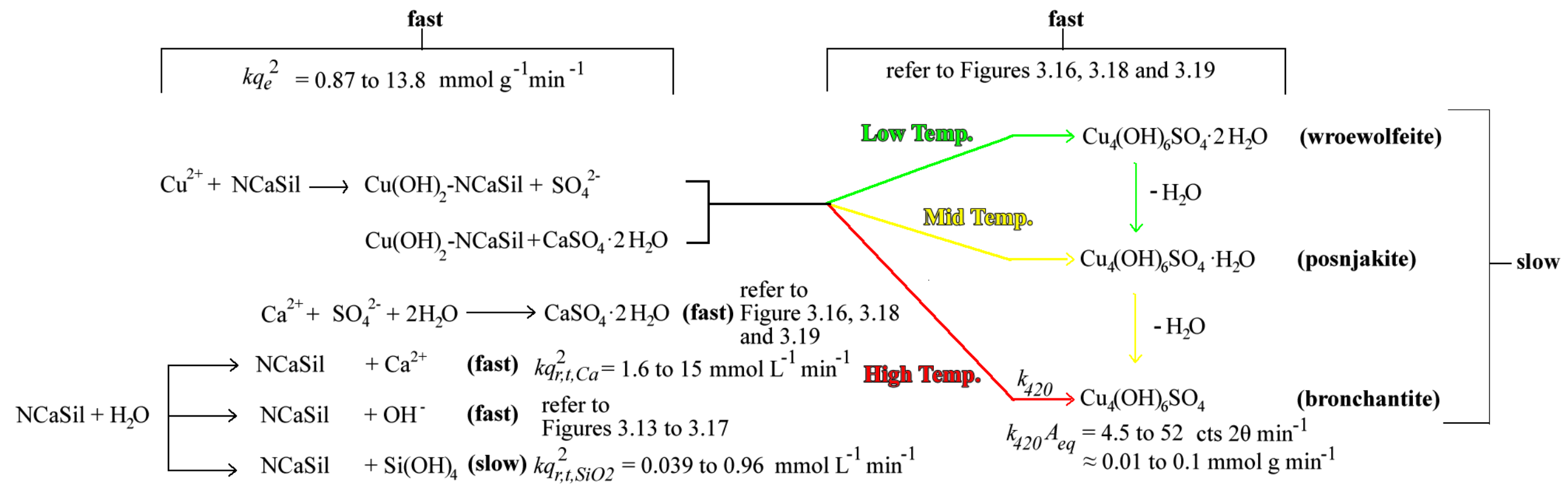

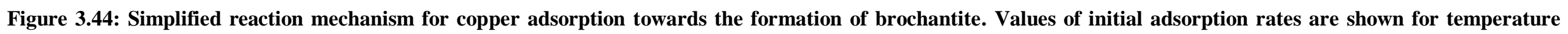

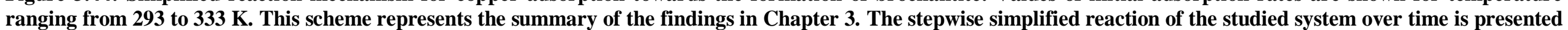

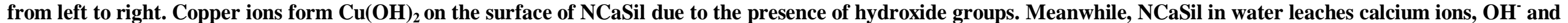

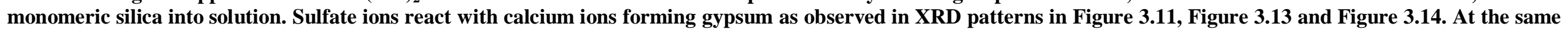

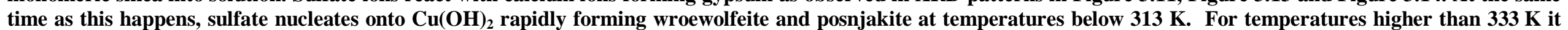
forms brochantite. Nonetheless at low temperatures the transformation of wroewolfeite and posnjakite into brochantite proved to be slow under the studied conditions. 
In addition the formation of wroewolfeite and posnjakite is fast at low (293 K) and mild $(313 \mathrm{~K})$ temperatures, while brochantite formation is fast at high temperatures (> $333 \mathrm{~K}$ ). The hydrolysis of NCaSil to form monomeric silica in solution is slower than all other reactions. Additionally, the dehydration of wroewolfeite and posnjakite to form brochantite was slow and took several hours to reach equilibrium at $293 \mathrm{~K}$. All kinetic rate constants were promoted to a similar extent by the input of heat into the system.

Analysing the kinetics of peak growth of brochantite at $333 \mathrm{~K}$, it is possible to observe that crystallographic plane 210 is the slowest to evolve from the studied planes within the brochantite crystal. Sulfate ions are located in this plane suggesting that the nucleation or relocation of this ion is the rate determining step towards the formation of brochantite.

The standard enthalpy, standard entropy and Gibbs free energy were calculated. Results show that the reaction is spontaneous at the studied temperatures, with a value for $\Delta \mathrm{G}$ at $293 \mathrm{~K}$ of $-26.1 \mathrm{~kJ} \mathrm{~mol}^{-1}$. The reaction is endothermic $\left(18 \mathrm{~kJ} \mathrm{~mol}^{-1}\right)$ and undergo a large entropy increase of $150 \mathrm{~J} \mathrm{~mol}^{-1} \mathrm{~K}^{-1}$. Consequently, the reaction becomes more spontaneous as the temperature increases. The increase of entropy was mainly due the large amount of monomers and oligomers of $\mathrm{SiO}_{2}$ released into solution when NCaSil was contacted with a $15.7 \mathrm{mmol} \mathrm{L}^{-1} \mathrm{Cu}^{2+}$ solution. The calculated values are most likely to reflect the variation in energies from the initial reagents in solution and the final step the brochantite formation including the release of species to solution.

Moreover, the amount of calcium release is almost independent of the temperature in contrast of monomeric silica which is enhanced by an increase in the temperature of the system. The amount of monomeric silica released was found to be limited to its solubility in solution.

It was found that at least a $\mathrm{pH}$ value of 3 is required in order to take up copper when a NCaSil dosage of $1 \mathrm{~g} \mathrm{~L}^{-1}$ is used.

From the experimental results obtained in this chapter we may assume that all $\mathrm{Cu}^{2+}$ ions in the solid phase will be constituents of brochantite crystals if enough time and energy is granted to the system under initial concentration of copper higher than $11.8 \mathrm{mmol} \mathrm{L}^{-1}$. 
Finally, from an industrial point of view of copper sulfate hydroxide minerals are of interest for the agrochemical industry as a fungicidal agent. Therefore, the best conditions to obtain such product would be to do a batch reaction containing $15.7 \mathrm{mmol} \mathrm{L}^{-1}$ copper sulfate solution at $293 \mathrm{~K}$ using a $1 \mathrm{~g} \mathrm{~L}^{-1}$ of NCaSil for 24 hours. Likewise, a concentration of $11.8 \mathrm{mmol} \mathrm{L}^{-1}$ and adsorbent dosage of $1 \mathrm{~g} \mathrm{~L}^{-1}$ at $303 \mathrm{~K}$ may be used as an alternative. Choosing either of these conditions will depend on the price of heating the solution against the market price of copper sulfate. 


\section{Chapter 4, Mining Waste Characterisation, Simulation and Treatment with NCaSil}

\subsection{Mining waste}

During mining operations large amounts of solid, liquid and even gaseous waste effluents are produced. It is estimated that every 1 tons of metallic copper produced, generates 350 tons of sterile rocks, 140 tons of low copper content rock, 66 tons of solid residues and 57 tons of lixiviation residues [87]. The process by which copper is produced varies depending on the type of mineral that is being extracted. Appendix A-4 to A-7 show flow sheets for the production of copper from different types of minerals.

Generally, copper production involves several steps aimed at releasing the copper containing mineral (usually copper sulfides) from the mined ore. Often grinding is the first step and subsequent steps involve the use of chemicals to release the element of choice. The flow sheets presented in appendix A-4 to A-7 show that multiple stages during the production of copper release liquid wastes (enclosed in red boxes), each of these have in common a low $\mathrm{pH}$ due to the presence of sulfuric acid in some of the stages of production. Arsenic is also very common in mining tailings since most copper sulfides are associated with arsenic bearing rocks [88].

New Zealand and Chile both have mining histories. While New Zealand moved from mining into agricultural production, Chile has given great importance of the exploitation of minerals providing the country with large revenues. Chile is the world's largest producer of copper which is mainly extracted in large scale mines but also in several other small and medium scale mines [89]. Large scale mines usually try to comply to 
international environmental management systems. Most companies have an ISO 14001 accreditation, to demonstrate that they take their responsibilities seriously [90]. In contrast small and medium scale mines do not follow international accreditation as it represents a huge investment of resources and is not compulsory, which could lead to a loose management of waste disposal.

Moreover, mining activity in New Zealand in the last century resulted in high metal ion content in river basins [91]. Often in the past after a mine was closed, no rehabilitation was carried out, leaving minerals exposed to oxidation and further leaching of metal ions into bodies of water. Wilson et al. [92] have reported that a historic antimony mine in Marlborough, New Zealand, contributed to an arsenic concentration three times larger in the leachate than in the groundwater entering the mine. Weber et al. [93] described that nickel was associated with acid rock drainage in coal mining activities in the West Coast of New Zealand. Gold mining in Reefton, New Zealand, has led to the leaching of arsenic out of rocks from the mining waste, although natural attenuation of the concentration downstream has been reported [94].

Traditional mining in the northern regions of Chile has led to the contamination of marine environments due to mining tailing discharges. Ramirez et al. [95] have reported the presence of metal ions in marine sediments on the coast near El Salvador, Chile, in the range of 7.2-985 mg kg${ }^{-1}$ of $\mathrm{Cu}$ and 746-22739 $\mathrm{mg} \mathrm{kg}^{-1}$ of Mn, respectively, which have been attributed to mining tailings. Some bays, such as la Herradura and Tongoy, have been reported to contain high concentrations of copper and iron ions [96]. The presence of the latter ion at Tongoy Bay was due to iron dust from the iron mineral loading wharf located in the bay, while the presence of copper ions could be associated to the fact that Tongoy was a copper port in the past.

\subsubsection{Acid mine drainage}

Acid Mine Drainage (AMD), also called Acid Rock Drainage (ARD), is a naturallyoccurring effluent produced when sulfide-bearing minerals are exposed to oxygen and water [46]. The generation of AMD is associated with iron sulfide containing rocks. This process can be enhanced by mining exposing more rock to air or by the presence of naturally occurring bacteria (e.g. thiobacillus ferrooxidans) [97] which accelerate the breakdown of sulfide ores. This is a common process in old abandoned mines where no terrain rehabilitation programme has been undertaken. It is difficult to estimate the potential for a mine to generate AMD effluents, but in most of the cases AMD is related to the following key factors present in sulfide ores: water or high humidity and oxidant 
availability (e.g oxygen). Presented below is the general mechanism by which AMD is generated from pyrite ore $[46,98]$.

Oxidation of the sulfide mineral in the presence of water,

$$
\mathrm{FeS}_{2}+3.5 \mathrm{O}_{2}+\mathrm{H}_{2} \mathrm{O} \rightarrow \mathrm{Fe}^{2+}+2 \mathrm{SO}_{4}{ }^{2-}+2 \mathrm{H}^{+}
$$

Further oxidation from ferrous to ferric ion occurs if the surrounding environment is sufficiently oxidizing,

$$
2 \mathrm{Fe}^{2+}+0.5 \mathrm{O}_{2}+2 \mathrm{H}^{+} \rightarrow 2 \mathrm{Fe}^{3+}+\mathrm{H}_{2} \mathrm{O}
$$

At $\mathrm{pH}$ values between 2.3 and 3.5, ferric ions precipitate as $\mathrm{Fe}(\mathrm{OH})_{3}$ and jarosite $\mathrm{KFe}_{3}\left(\mathrm{SO}_{4}\right)_{2}(\mathrm{OH})_{6}$, leaving little $\mathrm{Fe}^{3+}$ in solution while simultaneously lowering the $\mathrm{pH}$,

$$
\mathrm{Fe}^{3+}+3 \mathrm{H}_{2} \mathrm{O} \rightarrow \mathrm{Fe}(\mathrm{OH})_{3 \text { solid }}+3 \mathrm{H}^{+}
$$

The remaining $\mathrm{Fe}^{3+}$ may oxidize additional pyrite,

$$
\mathrm{FeS}_{2}+14 \mathrm{Fe}^{3+}+8 \mathrm{H}_{2} \mathrm{O} \rightarrow 15 \mathrm{Fe}^{2+}+2 \mathrm{SO}_{4}{ }^{2-}+16 \mathrm{H}^{+}
$$

The presence of sulfur degrading bacteria will catalyse the reaction by lowering the $\mathrm{pH}$ through the production of $\mathrm{H}_{2} \mathrm{SO}_{4}$ and preventing ferric hydroxide precipitation. Since the reaction involves the release of iron ions and other metal ions most AMD affected sites are often yellow to red in colour. In many cases the severity of AMD is related to the $\mathrm{pH}$ and is considered moderate in the range of $\mathrm{pH} 2-4$. Generally the characteristics of AMD change from site-to-site, but low $\mathrm{pH}$, high concentrations of iron ion as well as aluminium and manganese ions are often found. However, the concentration of heavy metal ions may vary depending on the minerals present at each site since solubility of metal ions is enhanced by low $\mathrm{pH}$ and oxidizing environments.

\subsubsection{Technologies to address mining waste waters}

There are several treatments for mining waste waters, but since all residual effluents are characterized by low $\mathrm{pH}$ values and a high metal ion content, most treatments include a basification stage raising the $\mathrm{pH}$ to a value near 9.5 [99]. The most common basification process involves the addition of lime and the use of settling ponds. An example of such a method is shown in appendix A-8. This method is low cost if enough space is available, but it has drawbacks since bad aeration leads to an incomplete removal of ferrous ions. This system also lacks flow control and is susceptible to wind which could possibly lead to a non-compliant discharge. Moreover, it is vulnerable to floods due to 
rainfall. A pumping station or gate system may be added but this increases the overall cost. Hence this is not the most desirable system.

When the ore is roasted in the furnace a gas stream is generated containing mainly $\mathrm{SO}_{2}$ and suspended solid particles rich in copper, arsenic and other metal ions. This gas stream is used to make concentrated sulfuric acid but it has to be cleaned in a prior step with water scrubbers and passed through an electrostatic precipitator in order to prevent the contamination of the catalyst used further in the process. A liquid effluent is generated at this stage which is commonly known as acid plant 'blow-down' or also referred to as weak acid in the literature [100-102]. This effluent usually has $\mathrm{pH}$ values $<1$ and high contents of arsenic, sulfate $\left(1-10 \mathrm{~g} \mathrm{~L}^{-1}\right)$ and transition metal-ions [101]. The term might have arises due to the generation of commercial sulfuric acid downstream of the process. The method to treat this "blow-down" effluent is shown in A-9 and is based on a precipitation step accompanied by the addition of a flocculant, facilitating the separation. This design helps to readily precipitate arsenic as $\mathrm{Ca}_{3}\left(\mathrm{AsO}_{3}\right)_{2}$ and $\mathrm{Fe}\left(\mathrm{AsO}_{3}\right)$. This offers an improvement over the pond method as it allows more control over the final characteristic of the resultant discharge effluent.

BAMAG $\mathrm{GmbH}$ has designed a variant of the former process making it possible to produce clean gypsum by utilizing the weak acid [101]. This helps to decrease the costs by generating a by-product and reducing the volume of the solid waste. A schematic of such a process is given in appendix A-10. Here the $\mathrm{pH}$ value is set to 1 with lime allowing the formation of $\mathrm{CaSO}_{4}$ and at the same time avoiding the precipitation of arsenic and transition metals. Once the product is formed, it is recovered by filtration and dewatered, being ready to be sold. The remaining effluent is treated under the standard scheme previously mentioned. This new method reports good results for weak acids containing a large amount of sulfates and low content of arsenic ions $<1 \mathrm{~g} \mathrm{~L}^{-1}$. The process has been implemented at Atlantic Copper, Huelva, Spain reducing the cost of waste disposal as expected.

Another chemical method available to remove metal ions is based on the precipitation of metal ions as sulfides by adding $\mathrm{NaS}_{2}$, NaHS or $\mathrm{H}_{2} \mathrm{~S}$. In the literature the latest process regarding this method involves the biogeneration of $\mathrm{H}_{2} \mathrm{~S}$ [103]. Here, the production of hydrogen sulfide is achieved from sulfate or sulfur through a bacterium catalyzed redox reaction. Usually acetic acid is used as the electron donor in the presence of desulfuromonas acetoxidans bacteria which grow using the energy released by linking the oxidation of acetate to the reduction of elemental sulfur. Eq. 4.5 and 4.6 
show the simplified equation for underlying reactions of sulfates and sulfur with acetic acid.

$\mathrm{SO}_{4}^{2-}+\mathrm{CH}_{3} \mathrm{COOH}+2 \mathrm{H}^{+} \rightarrow \mathrm{HS}^{-}+2 \mathrm{HCO}_{3}^{-}+3 \mathrm{H}^{+}$

$4 \mathrm{~S}^{0}+\mathrm{CH}_{3} \mathrm{COOH}+2 \mathrm{H}_{2} \mathrm{O} \rightarrow 4 \mathrm{H}_{2} \mathrm{~S}+2 \mathrm{CO}_{2}$

In appendix A-11 is shown a schematic of the process. The precipitation of metal ions as sulfides is often claimed to be superior compared to the ones involving the addition of lime in terms of sludge volume, reusability of the sludge and effluent quality. The advantages can be summarized as:

- High reactivity of sulfides with heavy metal ions and very low solubility of the metal sulfides over a broad $\mathrm{pH}$ range resulting in lower effluent concentrations.

- Sulfide precipitation, unlike hydroxide precipitation, is relatively insensitive to the presence of complexes and most chelating agents.

- The method can remove chromates and dichromates without preliminary reduction of the chromium to the trivalent state.

- A high degree of selective metal precipitation is achievable with sulfide, by controlling the $\mathrm{pH}$.

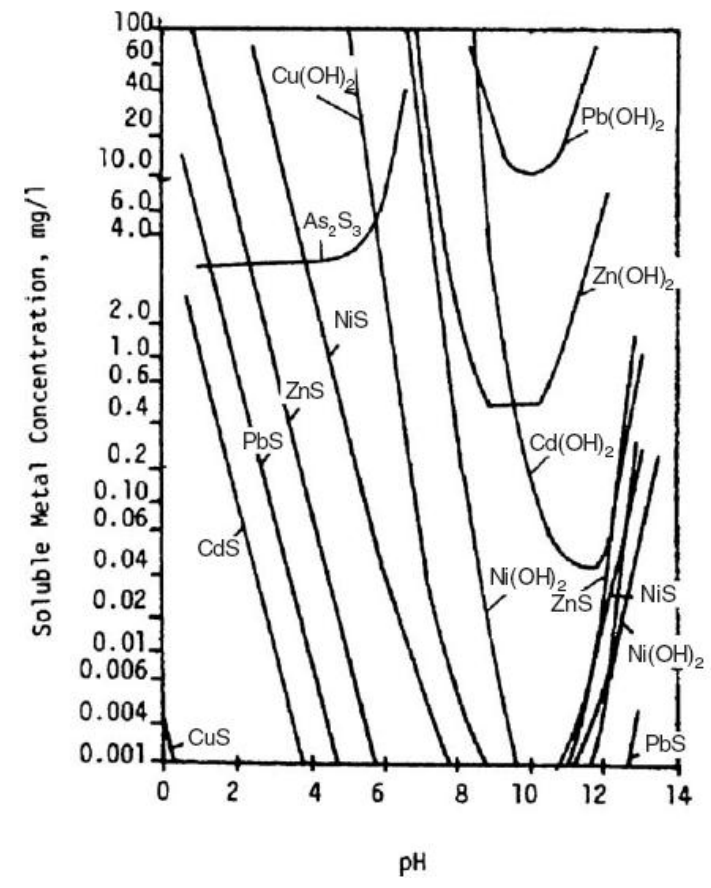

Figure 4.1: Comparison between metal hydroxides and metal sulfides equilibrium concentration taken from reference [103].

In Figure 4.1 is shown a speciation diagram comparing the equilibrium concentrations of metal hydroxides and metal sulfides. The solubility of metal sulfides is not only 
much lower but also it is effective over a wider range of $\mathrm{pH}$, thus having better control over the precipitation and generating a cleaner discharge effluent.

Metal sulfide sludges generally are easier to dewater and facilitate further processing due to a formation of a thicker sludge than metal hydroxides. This design, as discussed above, can selectively precipitate $\mathrm{CuS}$ and $\mathrm{NiS}$ separately by controlling the $\mathrm{pH}$ generating a reusable or sellable sludge. Drawbacks from this process come from the use of toxic reagents such as $\mathrm{H}_{2} \mathrm{~S}$ which involves the use of more complex equipment to avoid leakages.

It is possible to say that recent technologies aim to not only remove metal ions from the solution but also to generate a by-product that can be sold and help to reduce the cost involved in waste treatment and disposal. Hence, this chapter focuses on the recovery of $\mathrm{Cu}^{2+}$ ions from mining waste waters. Taking into consideration results obtained in Chapter 3, where high concentration of $\mathrm{Cu}^{2+}$ (higher than $3.9 \mathrm{mmol} \mathrm{L}^{-1}$ ) generated copper sulfate hydroxide species, the above mentioned weak acid presents an interesting waste to be studied and treated due to high concentrations of $\mathrm{Cu}^{2+}$.

\subsubsection{Weak acid production at El Teniente mine, Chile}

El Teniente is a copper mine operated by the state owned company Corporación Nacional del Cobre (CODELCO) located in the Andes mountains range near Rancagua in Chile. The mine extracts copper and molybdenum sulfides, which are separated by ore selective flotation. El Teniente is considered to be the world's largest underground copper mine in size and $5^{\text {th }}$ in terms of production of copper [100]. It operates two 'Teniente-type' furnaces, which are 22 meters long and 5 meters wide, located at Caletones $34^{\circ} 07^{\prime} \mathrm{S}$ and $70^{\circ} 27^{\prime} \mathrm{W}$ near the mining site, granting the mine a production capacity of 400,000 tons of copper per year [100].

Roasting of sulfide containing minerals generates gases with a high content of $\mathrm{SO}_{2}$ and suspended particulate material. The chemical composition and physical characteristics of this gaseous effluent are in direct relation to the mineral being roasted and the furnace employed. In the past, no post-treatment of the gases released from the furnaces was carried out, which led to the release of gases containing $\mathrm{SO}_{\mathrm{X}}$ and particulate material containing arsenic and heavy metals to the surrounding environments. The contribution to pollution derived from copper roasting in this particular region of Chile was investigated by Romo-Kröger et al. [104], who reached the conclusion that aerosols containing $\mathrm{Cu}, \mathrm{Zn}$, As and $\mathrm{S}$ were produced by the smelter, contributing significantly to the atmospheric contamination in the area. Another study showed that these aerosols 
were transported away from the site by wind currents and contributed to abnormal concentrations of heavy metal and metalloids in surrounding rivers and waterways [105].

In order to meet current environmental standards in Chile $[90,106]$ for the discharge of gases the exhaust from the smelter is thoroughly cleaned and sulfur is transformed into a valuable commodity before the remainder of the gases is released into the environment. Most sulfide smelters have a sulfuric acid plant attached to make use of the generated $\mathrm{SO}_{2}$ to produce sulfuric acid as a by-product. Commonly, sulfuric acid plants also help to recover heat from the exhaust current. In the case of El Teniente the gas is only cooled down and cleaned using a water mist without any attempt at recovering thermal energy being undertaken. During the gas-cleaning step, the exhaust gas is channelled through water scrubbing towers operating in counter current. As the gases cool down, particulate material and some of the $\mathrm{SO}_{2}$ including other gases are trapped in the condensate of the water vapour generating the weak acid. The next step of the exhaust purification involves an electrostatic precipitator, which further removes any fine particles suspended in the gas stream. This is important as the $\mathrm{SO}_{2}$ current must be free of any particulates before contacting it with a $\mathrm{V}_{2} \mathrm{O}_{5}$ catalyst which oxidizes $\mathrm{SO}_{2}$ to $\mathrm{SO}_{3}$, as particles could damage the catalyst or impede gas flow. Finally, the freshly generated $\mathrm{SO}_{3}$ is contacted with water to form concentrated sulfuric acid.

The copper sulfides targeted for copper mining at El Teniente are associated with arsenic containing ore. Hence, the weak acid from El Teniente is expected to contain high concentrations of arsenic and sulfate. The weak acid stream is regularly analysed for these species and for its acidity. The weak acid is treated in order to meet environmental discharge requirements described by Decreto Supremo 90 in Chile [14]. The chemical treatment of the weak acid consists of two stages. The first one involves the addition of milk of lime $\mathrm{Ca}(\mathrm{OH})_{2}$ to precipitate heavy metals as hydroxides $\mathrm{M}^{\mathrm{n}+}\left(\mathrm{OH}^{-}\right)_{\mathrm{n}}$, sulfate as gypsum $\mathrm{CaSO}_{4}$ and arsenic as calcium arsenite $\mathrm{CaAsO}_{3}$. The second stage involves the addition of ferric chloride and hydrochloric acid to precipitate any remaining arsenic from solution. The resulting precipitates form a sludge, which will be referred to in this work as 'dirty gypsum' and contains a mixture of calcium sulfate, calcium arsenite and ferric arsenite [102]. These inorganic salts have low solubility products. Therefore, they can be 'safely' disposed of on landfills with only minor leaching issues occurring.

Although weak acid is periodically surveyed at El Teniente to determine the content of $\mathrm{SO}_{4}{ }^{2-}$, As and suspended solids, little attention has been given to the presence of metals 
in both liquids (ions) and suspended solids (microparticles, nanoparticles) in the waste. This particulate material containing heavy metals could pose a threat to the environment. Even after being occluded by the dirty gypsum during the addition of milk of lime to the weak acid, these particles could be transported away by several mechanisms similar to those proposed by Romo-Kröger in his study [105].

In the literature most studies of the gaseous effluent have placed emphasis on the recovery of valuable metal ions from the flue dusts collected downstream at the electrostatic precipitator [107-109] and have neglected the weak acid waste. This work focuses on the acid plant 'blow-down', also known as weak acid, generated by water scrubbing towers. The characterisation of this waste will help to establish if it poses a hazard for the environment, and potential for metal recovery or treatment.

\subsubsection{Operation of cooling towers}

Weak acid samples were collected from the two cooling towers at El Teniente's sulfuric acid plant. Both towers produce an overall of $500 \mathrm{~m}^{3}$ day $^{-1}$ of weak acid with an average temperature at the outlet of $328 \mathrm{~K}$. Similar flows have been reported for other plants, for example, Toyo's smelter in Japan generates $300 \mathrm{~m}^{3}$ of weak acid per day [110].

The liquid was light blue in colour containing particulate material in suspension, which settled within minutes after sample collection. Although the liquid portion of the sample was light blue colour (characteristic of copper sulfate in solution), little attention has been paid to the metal content of this effluent in the past. A potential reason for this neglect is that the light blue colour becomes easily masked by the suspended solid particles, which lend fresh samples a grayish to light orange coloration. The other reason might be that the dissolved copper concentration of the effluent was estimated to be quite low, which makes this waste unattractive for hydrometallurgical processes and copper recovery.

CODELCO staff members provided operational data for the two water scrubber towers at the acid generation plant in El Teniente mine, collecting data daily over a period of 6 months. Appendix A-12 to A-17 show Stewart charts, also known as control charts for Plant 1 recording the total concentrations of arsenic, sulfate and weight percentages of suspended solids from May 2010 until October 2010. 
Table 4.1 presents the statistical parameters of the operation and summarizes the values presented in appendix A-12 to A-17. Values of $\pm 2 \sigma$, lower and upper warning limits (LWL and UWL), and $\pm 3 \sigma$, lower and upper action limits (LAL and UAL), for some of the parameters are not shown since they gave negative values and there cannot be negative concentrations. 
Table 4.1: Summary of the presented control chart parameters for the weak acid generated at Plants 1 and 2 at El Teniente.

\begin{tabular}{|c|c|c|c|c|c|c|}
\hline & \multicolumn{3}{|c|}{ Plant 1} & \multicolumn{3}{|c|}{ Plant 2} \\
\hline & $\begin{array}{c}\text { total As } \\
{\left[\mathrm{g} \mathrm{L}^{-1}\right]}\end{array}$ & $\begin{array}{c}\mathrm{SO}_{4}{ }^{-2} \\
{\left[\mathrm{~g} \mathrm{~L}^{-1}\right]}\end{array}$ & $\begin{array}{c}\text { suspended } \\
\text { solid } \\
\text { content } \\
{[\% \mathrm{wt}]}\end{array}$ & $\begin{array}{c}\operatorname{total} \text { As } \\
{\left[\mathrm{g} \mathrm{L}^{-1}\right]}\end{array}$ & $\begin{array}{c}\mathrm{SO}_{4}^{-2} \\
{\left[\mathrm{~g} \mathrm{~L}^{-1}\right]}\end{array}$ & $\begin{array}{c}\text { suspended } \\
\text { solid } \\
\text { content } \\
{[\% w t]}\end{array}$ \\
\hline Average & 6.1 & 43.2 & 0.23 & 6.5 & 68.7 & 0.97 \\
\hline Std. dev. & 2.9 & 10.0 & 0.63 & 1.2 & 11.6 & 0.95 \\
\hline $\min$ & 1.8 & 11.1 & 0.03 & 3.1 & 39.8 & 0.06 \\
\hline $\max$ & 65.0 & 73.8 & 13.00 & 11.5 & 108.5 & 9.82 \\
\hline UWL & 11.9 & 63.1 & 1.49 & 9.0 & 91.9 & 2.88 \\
\hline LWL & 0.3 & 23.2 & - & 4.0 & 45.5 & - \\
\hline UAL & 14.7 & 73.1 & 2.12 & 10.3 & 103.5 & 3.83 \\
\hline LAL & - & 13.2 & - & 2.8 & 33.9 & - \\
\hline
\end{tabular}

From Appendix A-12 to A-17 it can be seen that on the date when the samples studied throughout this article were collected, the plant was operating within $1 \sigma$ of the average, indicating that the samples collected present an insight into a normal operational day.

Plant 1 shows less data dispersion than Plant 2 for the total As and $\mathrm{SO}_{4}{ }^{2-}$ concentrations. On the $16^{\text {th }}$ of July, 2010, Plant 1 reported the maximum value for As during the monitored time with a concentration $65 \mathrm{~g} \mathrm{~L}^{-1}$ for this species in solution, as shown in 
Table 4.1. This value contributed to the large standard deviation of $2.9 \mathrm{~g} \mathrm{~L}^{-1}$ of arsenic for this plant. The average amount of suspended solids in Plant 2 is four times larger than in Plant 1. Furthermore, the concentration of $\mathrm{SO}_{4}{ }^{2-}$ reached a peak value of $108.5 \mathrm{~g} \mathrm{~L}^{-1}$ on the 11th of August 2010 (not shown in the graph). Despite these differences, overall, both plants operated steadily. There were 10 events where the parameters exceeded LAL or UAL values (located at $\pm 3 \sigma$ from the average) during the monitored period.

A study by Inami et al. [111] on the weak acid generated at the Toyo smelter, Japan, reported the following values for the major constituents: $80-150 \mathrm{~g} \mathrm{~L}^{-1} \mathrm{H}_{2} \mathrm{SO}_{4}$, $0.5-1 \mathrm{~g} \mathrm{~L}^{-1} \mathrm{Cu}, 2-5 \mathrm{~g} \mathrm{~L}^{-1} \mathrm{As}, 0.5-2 \mathrm{~g} \mathrm{~L}^{-1} \mathrm{Zn}$ and $1-5 \mathrm{~g} \mathrm{~L}^{-1} \mathrm{Cl}$. The concentration of arsenic and sulfate present in the supernatant of El Teniente's weak acid is higher compared to those reported for the one generated at Huelva, Spain and Toyo, Japan [101]. In the case of arsenic concentrations, the Chilean weak acid contains on average seven times the amount of arsenic compared to the concentrations used by BAMAG $\mathrm{GmbH}$ to emulate the Spanish weak acid [101]. El Teniente's average total As concentrations are 20 to $325 \%$ larger than those present in the weak acid from the Toyo's smelter. Furthermore, the $\mathrm{SO}_{4}{ }^{2-}$ concentration observed in the weak acid waste from El Teniente is approximately half of that present in the Toyo's smelter weak acid. These differences could be attributed to the quality of the ore being processed at the smelters, implying that Chilean ore has potentially a lower grade than the ore being roasted at the above mentioned Spanish and Japanese smelters. 


\subsubsection{Chemical characterisation of the liquid phase, the supernatant}

A representative sample of weak acid waste was collected by CODELCO at the sulfuric acid plant at El Teniente. The sample was filtered and the liquid phase was analyzed in a private laboratory in Chile. The results from the chemical analysis of the liquid phase of the sample are displayed in Table 4.2.

Table 4.2: Chemical analysis of the liquid portion of the sample.

\begin{tabular}{|c|c|c|}
\hline Analyte & unit & value \\
\hline $\mathrm{Al}^{3+}$ & & 113 \\
\hline As, total* & & 6.0 \\
\hline $\mathrm{Ca}^{2+}$ & & 1449 \\
\hline Co, total & & 0.103 \\
\hline Cr, total & & 0.076 \\
\hline $\mathrm{Cu}^{2+}$ & & 562 \\
\hline Fe, total & & 185 \\
\hline $\mathbf{K}^{+}$ & & 467 \\
\hline $\mathrm{Mg}^{2+}$ & $\zeta \mathrm{mg} \mathrm{L}^{-1}$ & 39.7 \\
\hline Mo, total & & 50.3 \\
\hline $\mathrm{Na}^{+}$ & & 152 \\
\hline $\mathrm{Ni}^{2+}$ & & 0.402 \\
\hline Pb, total & & 22 \\
\hline$V$, total & & $<0.008$ \\
\hline $\mathrm{Cl}^{-}$ & & 475 \\
\hline $\mathrm{NO}_{3}{ }^{-}$ & & $<0.20$ \\
\hline $\mathrm{SO}_{4}{ }^{2-}$ & & 32800 \\
\hline Acidity & $\mathrm{mg} \mathrm{L}^{-1} \mathrm{CaCO}_{3}$ & 2970 \\
\hline COD & $\mathrm{mg} \mathrm{L}^{-1}$ & 3023 \\
\hline Conductivity & $\mu \mathrm{S} \mathrm{cm}$ & 334000 \\
\hline pH & & 0.45 \\
\hline
\end{tabular}

Table 4.2 shows that the major components, with concentrations $>100 \mathrm{mg} \mathrm{L}^{-1}$, in the weak acid are $\mathrm{H}^{+}, \mathrm{Cu}^{2+}, \mathrm{SO}_{4}{ }^{2-}, \mathrm{Ca}^{2+}$, total $\mathrm{Fe}$, total $\mathrm{As}, \mathrm{K}^{+}$, and $\mathrm{Al}^{3+}$. Consequently, this liquid waste can be categorized as extremely acidic and high in metal content according to the Flicklin diagram [112]. The low $\mathrm{pH}$ value of 0.45 may be explained from the formation of sulfurous acid and sulfuric acid due to the presence of $\mathrm{SO}_{2}$ in solution. The reactions of $\mathrm{SO}_{2}$ in presence of water in anoxic and oxidizing conditions are represented in eq. 4.7 and eq. 4.8 respectively,

$$
\begin{aligned}
& \mathrm{SO}_{2(\mathrm{~g})}+\mathrm{H}_{2} \mathrm{O} \rightarrow \mathrm{H}_{2} \mathrm{SO}_{3} \\
& \mathrm{SO}_{2(\mathrm{~g})}+1 / 2 \mathrm{O}_{2(\mathrm{~g})}+\mathrm{H}_{2} \mathrm{O} \rightarrow \mathrm{H}_{2} \mathrm{SO}_{4}
\end{aligned}
$$


Taking into consideration that both of these acids are polyprotic in nature, the degree of protonation of the acid in solution will depend on their $\mathrm{pK}_{\mathrm{a}}$. Sulfurous acid has a $\mathrm{pK}_{\mathrm{a}}$ value for the first deprotonation of 1.90 and for the second deprotonation of 7.29. Therefore it is most likely that the species found in solution will be $\mathrm{H}_{2} \mathrm{SO}_{3}$. On the other hand, sulfuric acid has a $\mathrm{pK}_{\mathrm{a}}$ value for the first and second deprotonation of -3.0 and 1.99 respectively, so it is most likely that the predominant species present in solution will be $\mathrm{HSO}_{4}{ }^{-}$. For this study it was assumed that a mixture of $\mathrm{H}_{2} \mathrm{SO}_{3}$ and $\mathrm{HSO}_{4}{ }^{-}$was present in the sample.

The high value for the Chemical Oxygen Demand (COD) of $3023 \mathrm{mg} \mathrm{L}^{-1}$ may be attributed to the presence of $\mathrm{As}^{3+}$ that can be oxidized to $\mathrm{As}^{5+}$. Also, $\mathrm{SO}_{3}{ }^{2-}$ may contribute to the COD considering that it can be oxidized to $\mathrm{SO}_{4}{ }^{2-}$.

Taking into consideration the volume of weak acid produced per day and the concentration reported in Table 4.2, it can be calculated that approximately 100 tons of copper are expelled through the fumes of this copper smelter into the weak acid waste per year. Considering the actual copper price (6.5 USD kg-1) this presents a significant loss of the metals and economical value. At Toyo's smelter, copper ions present in the weak acid are recovered as copper sulfide by the addition of NaHS [110]. 


\subsubsection{Characterisation of the solid phase: suspended particles.}

Results from the chemical analysis of the digested sample using FAAS and ICP-OES are shown in Table 4.3.

Table 4.3: Concentration of different metals and metalloids found in the suspended solids of the weak acid waste collected at El Teniente.

\begin{tabular}{ll}
\hline Analyte & \multicolumn{1}{c}{$\begin{array}{c}\text { Concentration } \\
{\left[\mathbf{m g ~ g}^{-1}\right]}\end{array}$} \\
\hline $\mathbf{A l}$ & $0.2480 \pm 0.0006$ \\
$\mathbf{A s}$ & $4.765 \pm 0.019$ \\
$\mathbf{B a}$ & $<\mathrm{LOD}$ \\
$\mathbf{B i}$ & $3.493 \pm 0.010$ \\
$\mathbf{C a}$ & $2.492 \pm 0.052$ \\
$\mathbf{C o}$ & $0.08 \pm 0.078$ \\
$\mathbf{C r}$ & $<\mathrm{LOD}$ \\
$\mathbf{C u}$ & $10.04 \pm 0.34$ \\
$\mathbf{F e}$ & $6.48 \pm 0.29$ \\
$\mathbf{H g}$ & $0.2170 \pm 0.0007$ \\
$\mathbf{K}$ & $32.20 \pm 0.15$ \\
$\mathbf{M g}$ & $0.04900 \pm 0.0003$ \\
$\mathbf{M n}$ & $0.048 \pm 0.0387$ \\
$\mathbf{M o}$ & $<\mathrm{LOD}$ \\
$\mathbf{N a}$ & $0.722 \pm 0.008$ \\
$\mathbf{N b}$ & $0.736 \pm 0.712$ \\
$\mathbf{P b}$ & $635 \pm 17$ \\
$\mathbf{R e}$ & $<\mathrm{LOD}$ \\
$\mathbf{S b}$ & $0.868 \pm 0.004$ \\
$\mathbf{S n}$ & $0.1100 \pm 0.0006$ \\
$\mathbf{T i}$ & $0.179 \pm 0.014$ \\
$\mathbf{V}$ & $0.416 \pm 0.004$ \\
$\mathbf{Y}$ & $<\mathrm{LOD}$ \\
$\mathbf{Z n}$ & $0.643 \pm 0.104$ \\
$\mathbf{Z r}$ & $0.608 \pm 0.003$ \\
\hline
\end{tabular}

$\mathrm{Cu}, \mathrm{Fe}$, and $\mathrm{Pb}$ were measured using FAAS, all other analytes were measured in ICP-OES.

The highest concentration is that of lead with a concentration of $635 \pm 17 \mathrm{mg} \mathrm{g}^{-1}$. Followed by a fair amount of potassium, copper and iron in the sample with concentrations of $32.20 \pm 0.15,10.04 \pm 0.34$ and $6.48 \pm 0.29 \mathrm{mg} \mathrm{g}^{-1}$ for each metal respectively. Arsenic and bismuth were present in concentrations of $4.765 \pm 0.019$ and $3.493 \pm 0.010 \mathrm{mg} \mathrm{g}^{-1}$, respectively. On the other hand, barium, chromium, molybdenum, rhenium, silicon and yttrium were below the limit of detection (LOD) of the analysis methods employed. The proportions of all other analytes were between the LOD and 1 $\mathrm{mg} \mathrm{g}^{-1}$. 
High concentrations of lead might be explained considering that the ore molybdenite $\left(\mathrm{MoS}_{2}\right)$ often occurs in combination with small amounts of wulfenite $\left(\mathrm{PbMoO}_{4}\right)$. This is consistent with molybdenum being present in the liquid phase of the sample as shown in Table 4.2. Also galena, a lead sulfide ( $\mathrm{PbS})$, should be considered as a possible source. It is likely that when molybdenum sulfide is separated from copper sulfides by selective ore flotation, wulfenite stays with the copper sulfides as wulfenite and/or galena are less hydrophobic (different wettability) than the molybdenite. As a result, lead will be released when copper ore is roasted in the smelter. This is supported by the concentration of molybdenum in solution $\left(50 \mathrm{mg} \mathrm{L}^{-1}\right)$.

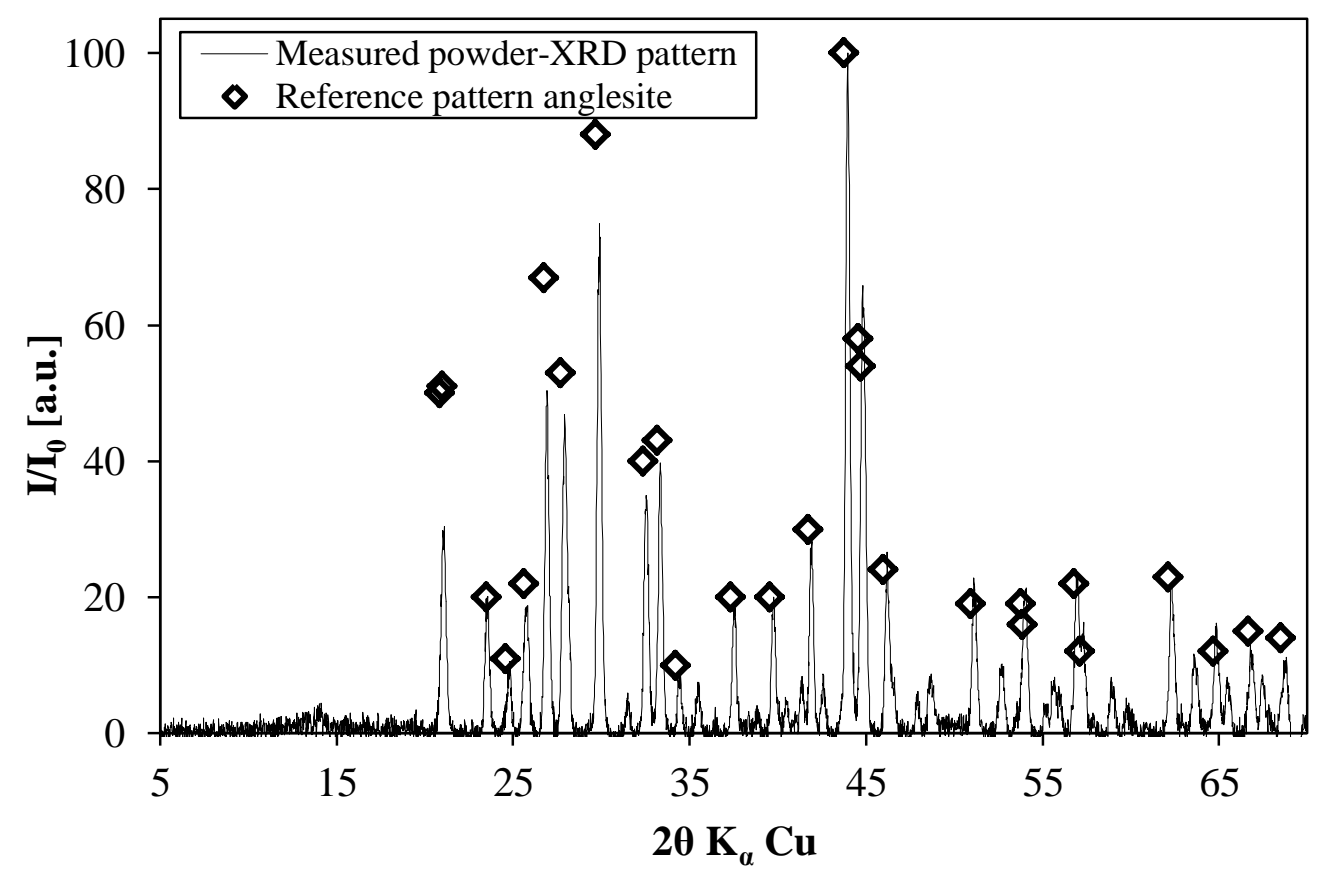

Figure 4.2: XRD pattern for the suspended solids from the weak acid sample. Refer to section 2.4.1 for sample collection.

A small dried sample of the suspended solids of the weak acid was analysed using powder-XRD. Figure 4.2 shows the retrieved pattern. The reflections fitted those of anglesite $\left(\mathrm{PbSO}_{4}\right)$ (PDF FILE 00-036-1461) scoring 85\% in the search and match option of the PANALYTICAL data processing software. This is in accordance with other findings where the anglesite is reported to form in the exhaust during the smelting of ore containing lead [113]. Some of the remaining reflections fitted compounds such as arsenic trioxide and iron sulfite. However, the score for the fit of these structures was very low even after subtraction of the anglesite pattern. Some of the standard patterns on record were of too low quality to unambiguously identity specific structures. Specifically, reflections associated with arsenic trioxide were discarded, as the structure of this compound is polymorphous. 


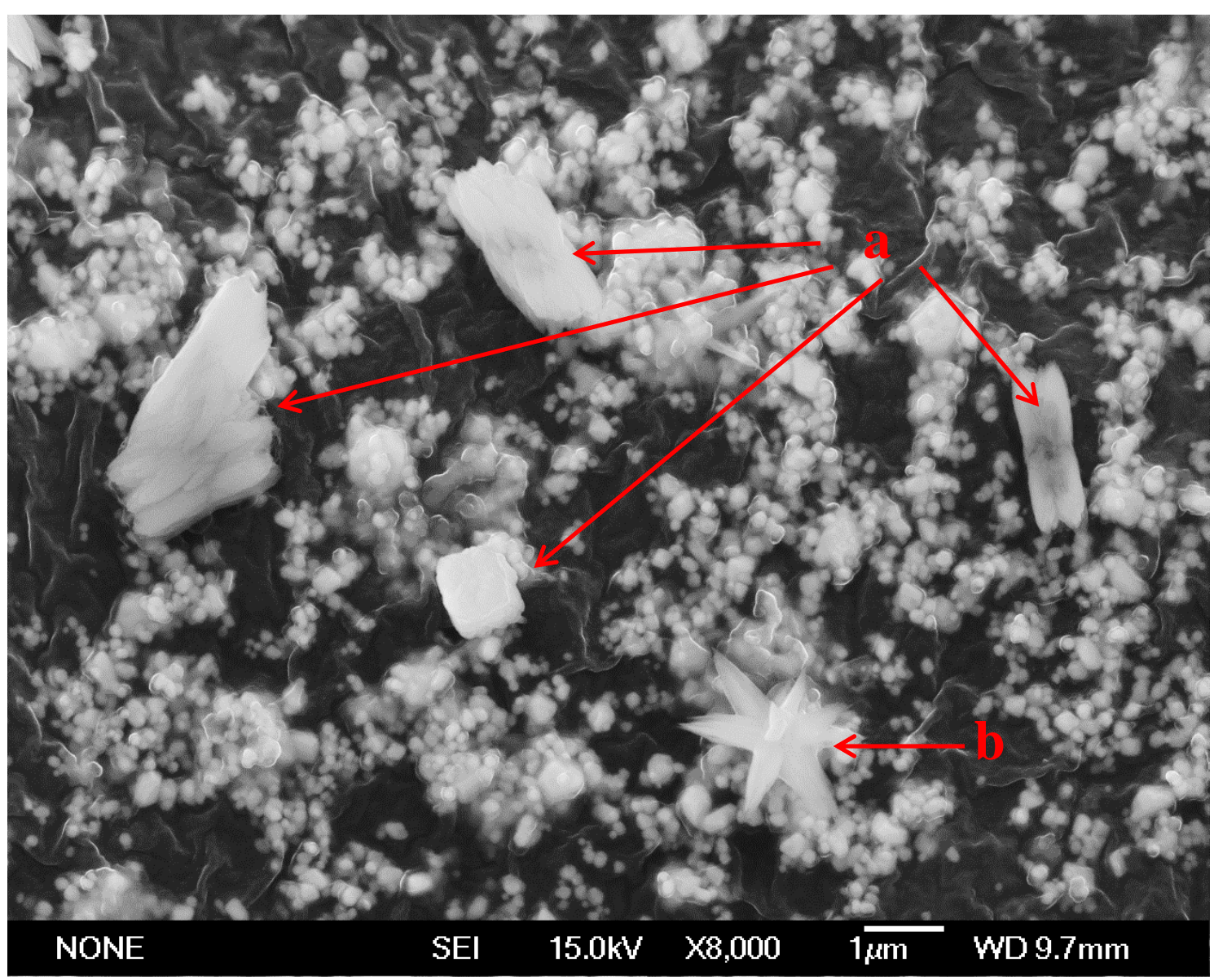

Figure 4.3: Three different types of structures observed in the suspended solids: a) rectangular rhomboids; b) star shapes; and unlabeled sub-micron particles (bright, white dots and clusters of dots). Refer to section 2.4 .1 for sample colletion

Scanning electron microscopy (SEM) analysis revealed three types of solid structures. The largest structure observed was a rhomboid as shown in four spots (marked as a) in Figure 4.3) in the backscattered image of the dried suspended solids sample. This structure was composed mainly of sodium, sulfur and oxygen as shown by energy dispersive spectroscopy (EDS) and associated X-ray maps (Figure 4.4). The high correlation of the three elements represented as white in the convoluted representation indicated that this structure probably is crystalline sodium sulfate. There was no evidence for crystals of sodium sulfate in the XRD pattern of the suspended solids. Considering the solubility of sodium sulfate, it was thought that these crystals formed during the drying step of sample preparation from the liquid films covering the suspended solids, as said solids were not washed. 


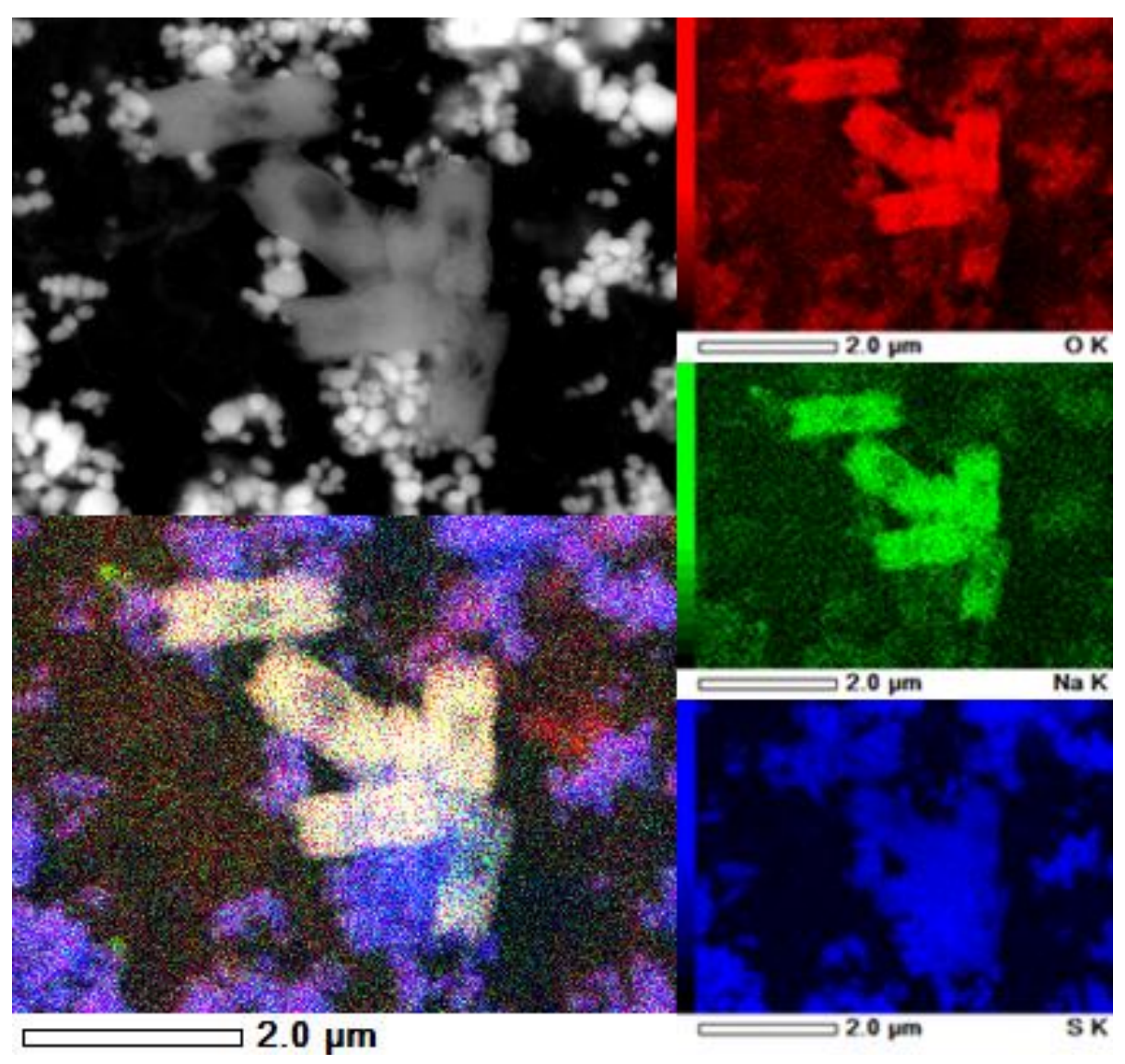

Figure 4.4: SEM-EDS imaging of the rhomboid type of structures found in the suspended solids. Presence of sulfur is shown in blue, oxygen in red and sodium in green. The overlay of oxygen and sulfur is represented as purple and the overlay of oxygen, sulfur and sodium as white. A backscattered electron image is presented in grayscale in the top left corner for orientation.

The second type of structure corresponded to a star shaped particle as shown in the spots marked as b) in Figure 4.3. It had a high content of arsenic, calcium and oxygen as evidenced by EDS. An X-ray overlay map produced using the EDS data showing the structure in white colour in the convoluted representation in Figure 4.5, suggesting it to be a calcium arsenate crystal. This could not be corroborated by XRD as the pattern did not match calcium arsenate peaks. The star-shaped particles were unstable and suffered irreversible damage during electron microscopy, which suggests that the particle might have lost the water molecules bound in their lattice. It is likely that calcium arsenate was present in the suspended solids in amorphous form and only crystallized and decomposed during sample preparation and analysis. 


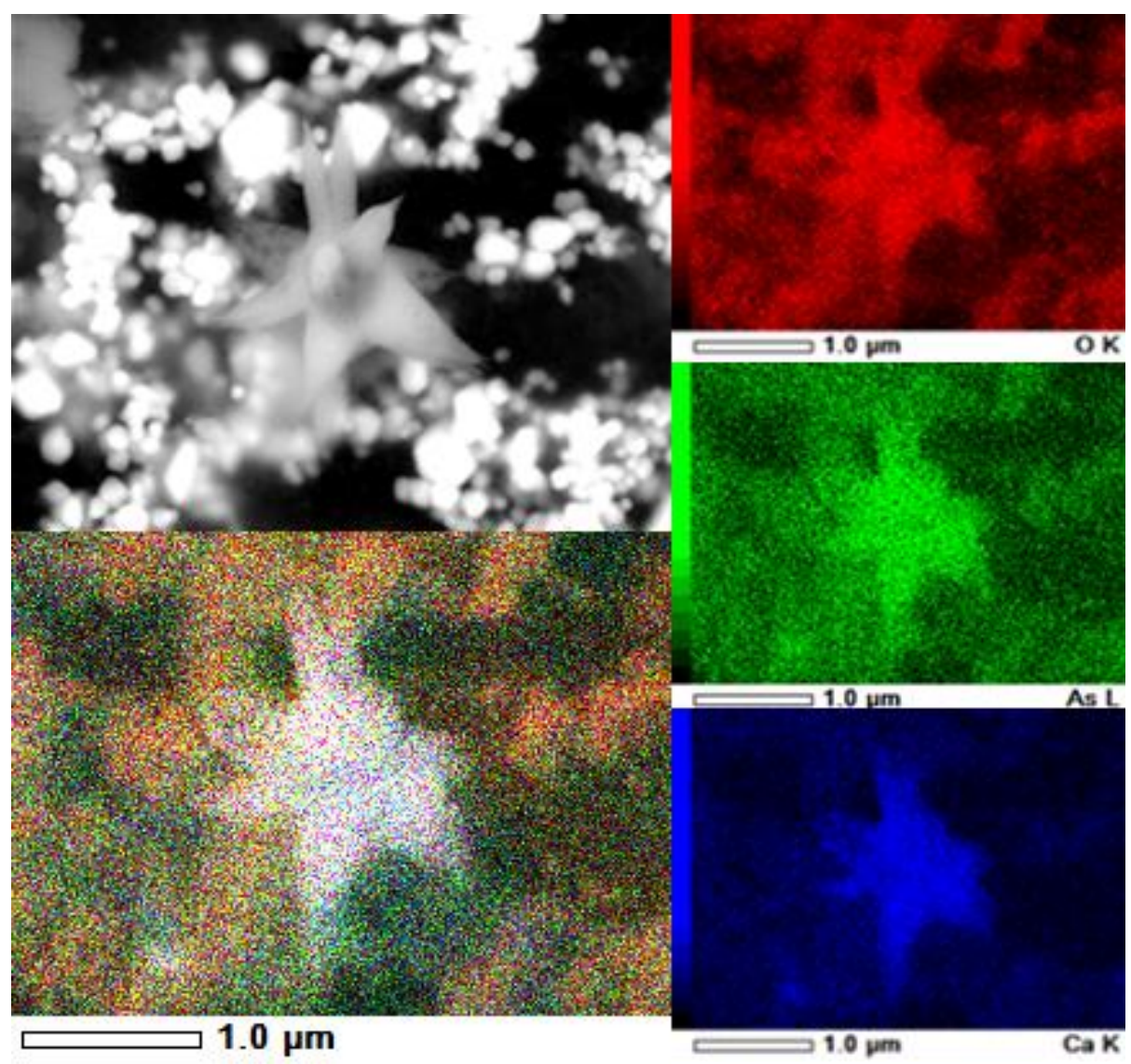

Figure 4.5: SEM-EDS imaging of the star shaped type of structures observed in the suspended solids. Presence of calcium is represented in blue, oxygen in red and arsenic in green. The overlay of these three elements is shown in white. A backscattered electron image is presented in grayscale in the top left corner for orientation.

The third type of particles represents the predominant species found in the suspended solids. These particles are sized in the range from 50-500 $\mathrm{nm}$. These sub-micron particles are noticeable in Figure 4.3 as bright, white dots and clusters of dots. Considering that commonly used microfiltration membranes in chemical analysis have a cut-off point of $0.45 \mu \mathrm{m}$, it is most likely that these particles bypassed any filtration and gas purification steps carried out in the sulfuric acid plant. It is also likely that some of these sub-micron particles will have remained in the filtrate and, hence, contributed to the quantification of sulfate in solution during the analysis presented earlier. The images of the sub-micron particles present them in a strong contrast to the background, especially when the backscattered electron detector of the SEM is employed (Figure 4.3). This suggests that the constituents are elements of high atomic mass. Figure 4.3 shows the difference in the contrast between the background and the flower type crystals, the rhomboid crystals and the sub-micron particles. Furthermore, EDS analysis in Figure 4.6 suggests that the sub-micron particles are mainly composed of lead, sulfur and oxygen. XRD results shown in Figure 4.2 confirm this, indicating that the main component of the suspended solids of the weak acid is anglesite $\left(\mathrm{PbSO}_{4}\right)$. Moreover, the 
third type of particles did not suffer structural changes during any of the analysis methods suggesting they were formed under high temperature conditions.

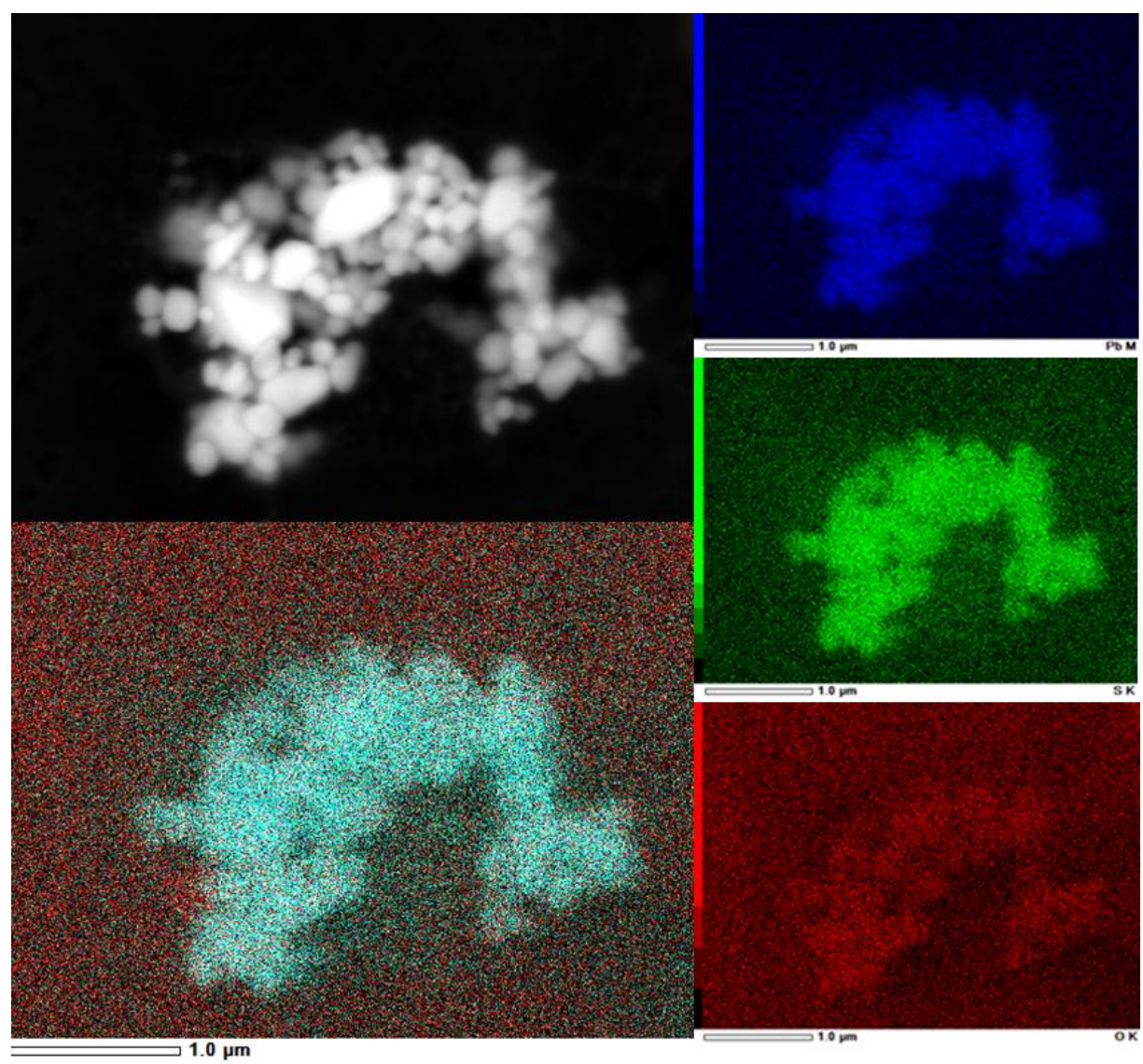

Figure 4.6: SEM-EDS imaging of the sub-micron particles. Lead is represented as blue, oxygen as red and sulfur as green. The overlay of oxygen, sulfur and lead mapping is represented in light blue. A backscattered electron image is presented in grayscale in the top left corner for orientation.

The findings of this study differ from those obtained by Roca et al. [107] reporting on the flue dust coming from flash smelting furnace. The collected particles were of a complex structure containing multiple mainly copper based minerals such as cuprospinel $\left((\mathrm{Cu}, \mathrm{Mg}) \mathrm{Fe}_{2} \mathrm{O}_{4}\right)$, chalcocyanite $\left(\mathrm{CuSO}_{4}\right)$, delafossite $\left(\mathrm{CuFeO}_{2}\right)$, zincosite $\left(\mathrm{ZnSO}_{4}\right)$, claudetite $\left(\mathrm{As}_{2} \mathrm{O}_{3}\right)$, arsenolite $\left(\mathrm{As}_{2} \mathrm{O}_{3}\right)$ and tenorite $(\mathrm{CuO})$. Furthermore, some particles found in the flue dust were up to two orders of magnitude larger than the ones present in El Teniente's weak acid presented here. Roca et al found that the particles contained a high concentration of copper that could be easily leached out with water. In contrast to their findings, the chemical analysis revealed that the solid phase of the sample collected at El Teniente contained, as was shown in Table 4.3, a considerably smaller quantity of copper. Taking into consideration the concentration of copper in the solid phase $\left(10.04 \pm 0.34 \mathrm{mg} \mathrm{L}^{-1}\right)$ and comparing it to the liquid phase $\left(562 \mathrm{mg} \mathrm{L}^{-1}\right)$ of El Teniente's weak acid, one might assume that copper ions present in solution leached out from particles, when they were surrounded by water inside the cooling tower. 
From an environmental and toxicological point of view, the solid phase represents a serious hazard, not only due to its high concentration in lead but also due to the fact that it is composed of sub-micron particles. It is most likely that upon treatment of the weak acid these sub-micron particles are occluded in the 'dirty gypsum'. The immobilization of the sub-micron particles inside the gypsum has to be established. In the case that it is easy to dislodge them from the gypsum, sub-micron particles could easily be transported away from the dirty gypsum landfill to pose a health or environmental threat due to their particle size.

In a different aspect, CODELCO reported difficulty in the first stage of the weak acid treatment, presenting slow kinetics in the precipitation of $\mathrm{SO}_{4}{ }^{2-}$ as calcium sulfate. Usually, an excess of milk of lime had to be used in order to precipitate this anion. This could be due to sulfate being present to a large extent as colloidal sub-micron particles. This means that instead of precipitating $\mathrm{SO}_{4}{ }^{2-}$ ions from solution (homogeneous reaction), represented in eq. 4.9 , calcium would also have to react with the surface of the sub-micron particles (heterogeneous reaction) as represented in eq. 4.10. The consequence is that the formation and precipitation of gypsum is considerably slower than expected and results in a poorer product containing sub-micron particles of lead sulfate and lead impurities.

$$
\begin{aligned}
& \mathrm{Ca}^{2+}+\mathrm{SO}_{4}{ }^{2-} \rightleftharpoons \mathrm{CaSO}_{4(\mathrm{~s})} \\
& \mathrm{Ca}^{2+}+\mathrm{PbSO}_{4(\mathrm{~s})} \rightleftharpoons \mathrm{CaSO}_{4(\mathrm{~s})}+\mathrm{Pb}^{2+}
\end{aligned}
$$

Even if the reaction displayed in eq. 4.10 occurs, an excess of calcium would have to be added in order to displace the equilibrium towards the formation of calcium sulfate, as the $K_{s p}$ of lead (II) sulfate $\left(1.7 \times 10^{-8}\right)$ is three orders of magnitude smaller than the $K_{s p}$ of calcium sulfate $\left(2.4 \times 10^{-5}\right)$. Considering this, the possibility that colloidal sub-micron particles are removed from solution by occlusion within the dirty gypsum sludge is very likely to occur.

\subsubsection{Treatment of an emulated mining waste}

Weak acid was chosen due to the high concentration of copper present. Other mining related effluents such as AMD do not contain copper above $100 \mathrm{mg} \mathrm{L}^{-1}$. An emulated version of the waste was prepared using the major dissolved constituents of this waste as described in Section 2.4. 

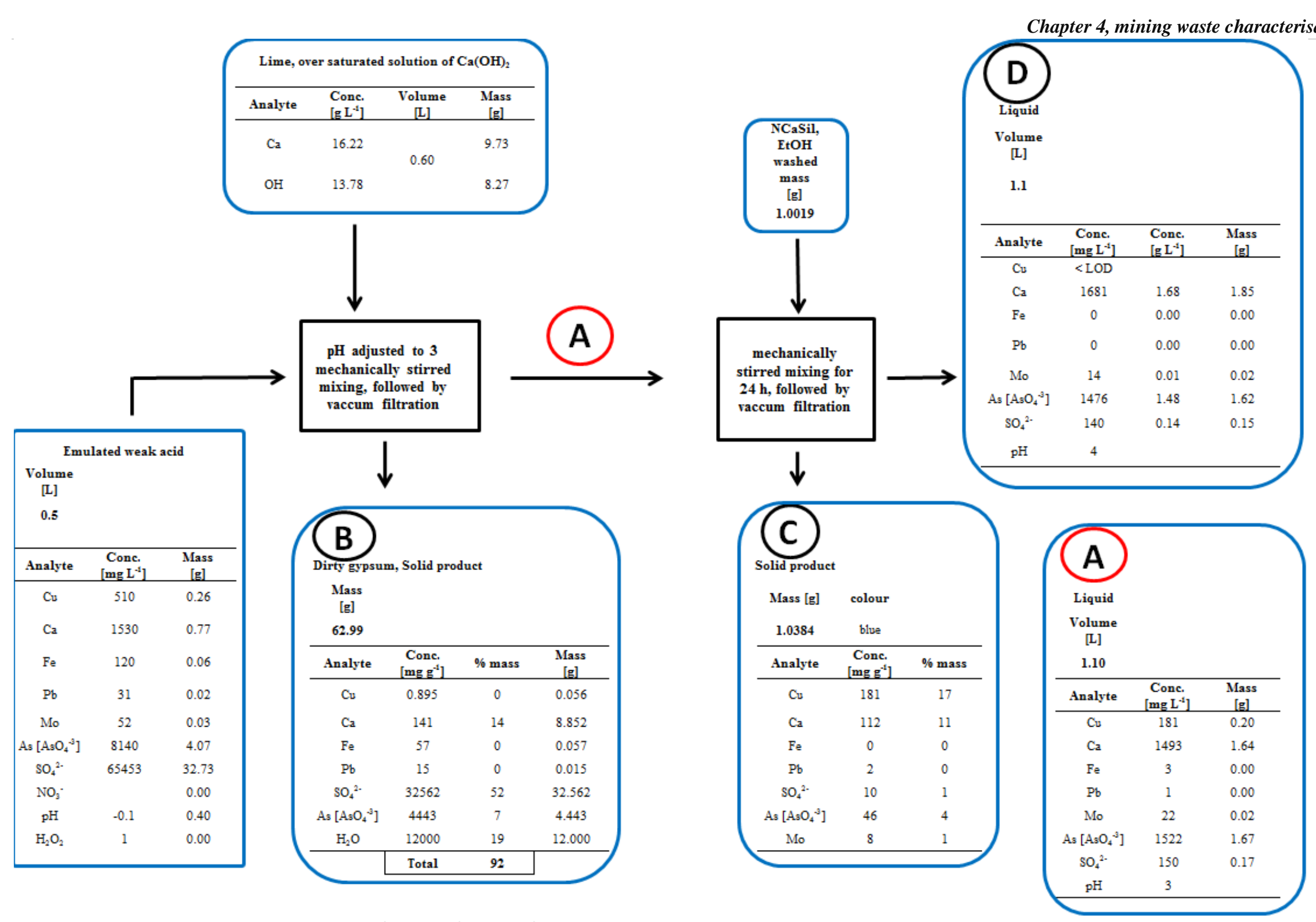

Figure 4.7: Emulated mining waste treatment flow sheet. 
The emulated weak acid was treated to raise the $\mathrm{pH}$ to a value of 3 . This step is important since all hydroxide groups on $\mathrm{NCaSil}$ are neutralized under this $\mathrm{pH}$, thus not forming copper hydroxide on the surface. Additionally raising the $\mathrm{pH}$ above 3 may cause the precipitation of copper as a hydroxide salt.

This steps proves the possibility of attaching a NCaSil production plant and adsorption batch reactors to the previously mentioned BAMAG process. First it would include the precipitation of clean gypsum at $\mathrm{pH} 1$ with a subsequent stage of raising the $\mathrm{pH}$ to 3 to produce the copper rich calcium silicate product. Hence two products, with commercial potential, would be produced on one hand clean gypsum and on the other a copper-rich calcium silicate. A scheme of such process is presented in appendix A-19

The addition of lime was expected to form insoluble compounds such as calcium hydrogen sulfate $\mathrm{Ca}\left(\mathrm{HSO}_{4}\right)_{2}$ and calcium hydrogen arsenate $\mathrm{Ca}\left(\mathrm{HAsO}_{4}\right)$ considering the $K_{s p}$ and $\mathrm{pK}_{\mathrm{a}}$ value for each of these salts. Nevertheless, other variation of the above salts could be formed such as calcium sulfate and calcium arsenate. The sample is called dirty gypsum mainly due to its content of arsenic making it useless for industrial application. In this case it was 52\% wt of sulfate, $19 \%$ wt water, $14 \%$ wt of calcium and $7 \%$ arsenic as arsenate. In fact, $99.8 \%$ of the sulfate was removed from solution in this stage. Nonetheless, only $81 \%$ of the initial amount of arsenate was removed. Additionally, $50 \%$ of the initial mass of iron and lead was removed from solution. In the case of copper only $20 \%$ of the initial mass was removed from solution. 
Figure 4.8 shows a SEM image at 2000x magnification of a solid sample of the filter cake recovered in stage B of Figure 4.7.

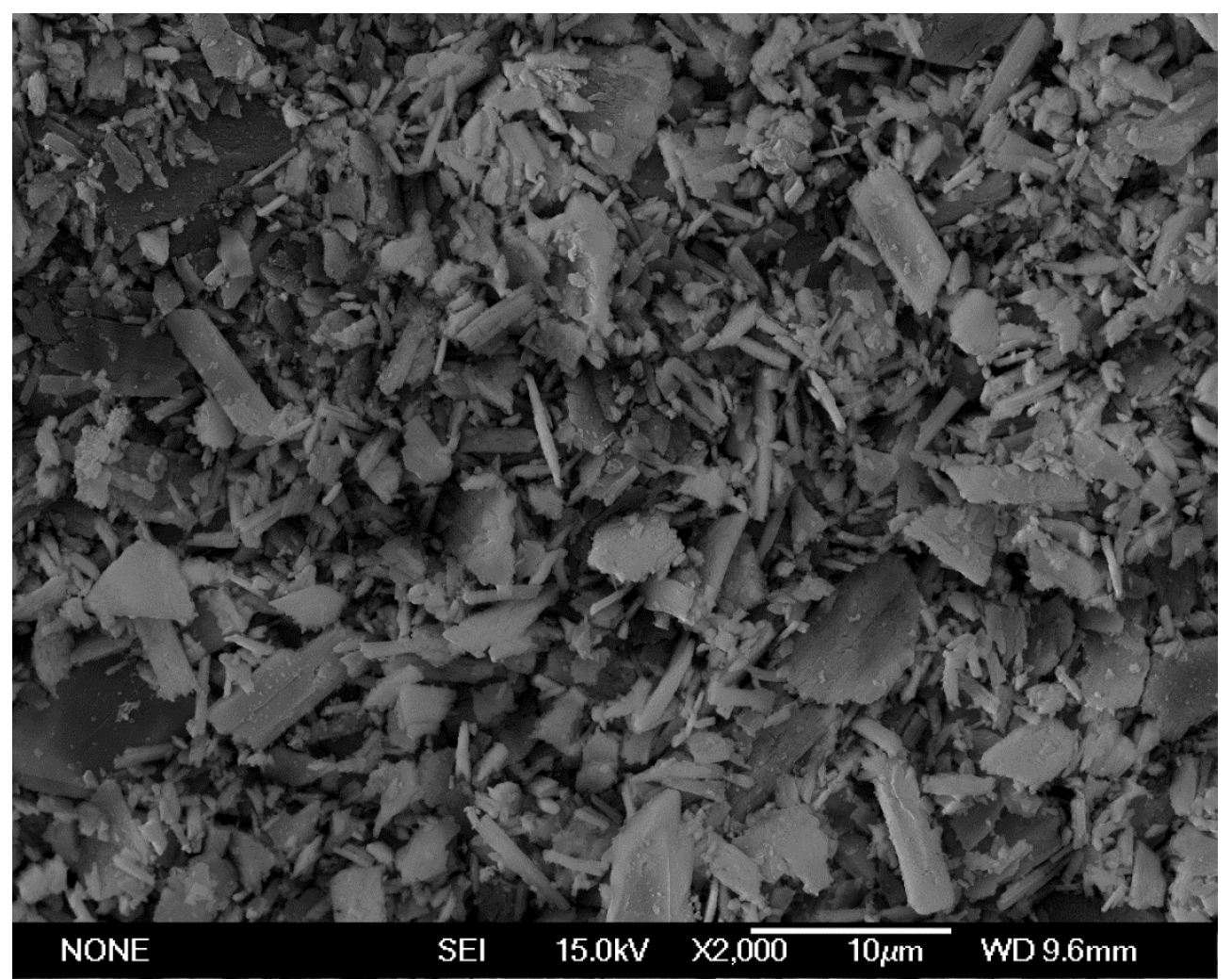

Figure 4.8: SEM image of the dirty gypsum produced at stage $B$ in Figure 4.7.

The sample was covered by the flake-type morphology shown in the scanned region in Figure 4.8. Probably these crystals correspond to distorted prisms ranging in the size of a fraction of micrometre to 10 micrometre in length.

A backscatter image of the same region in Figure 4.9 shows that the atomic mass of the constituents is even throughout the surface of the sample. Nevertheless, there are some slightly brighter spots which are enclosed in white boxes in the picture. 


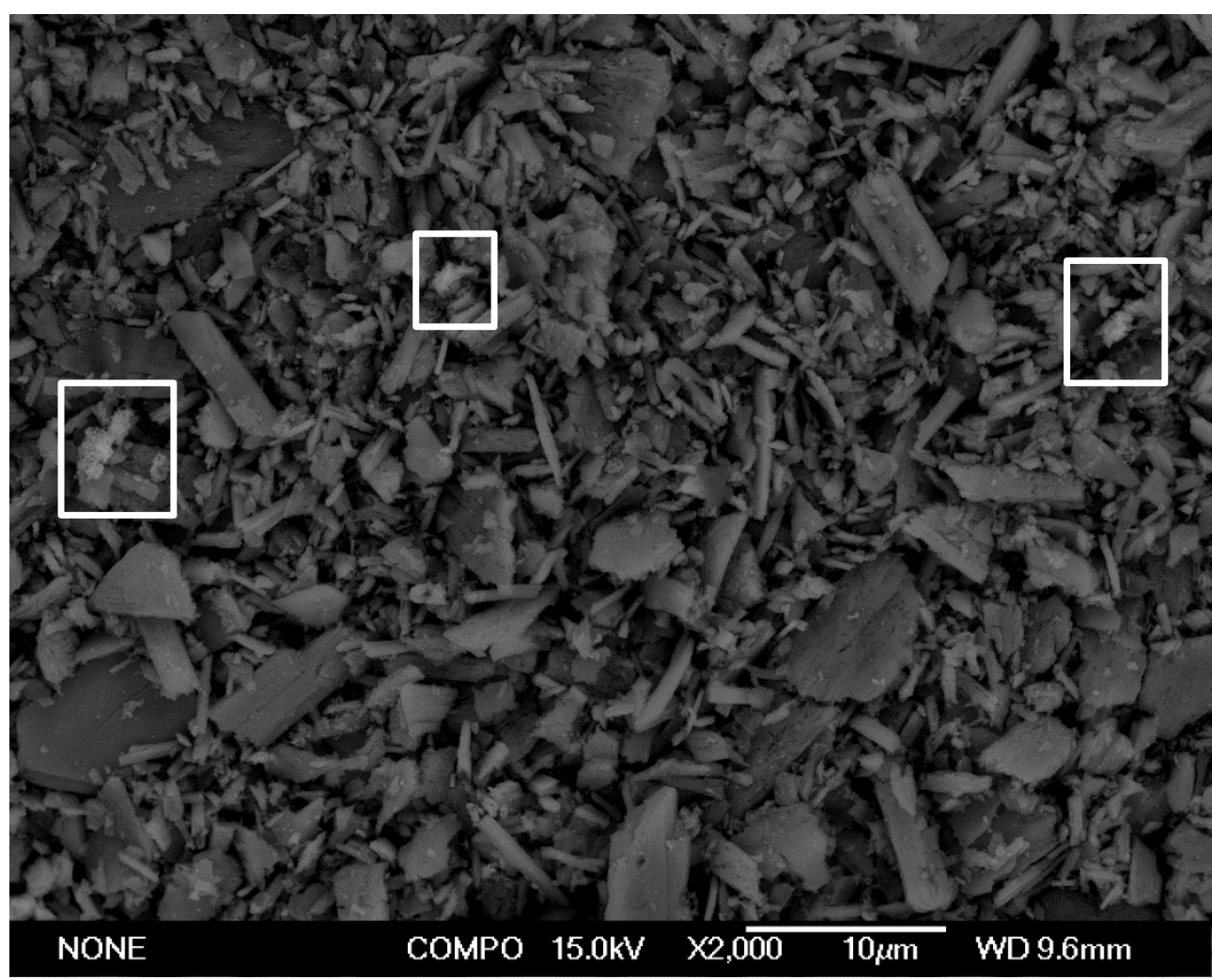

Figure 4.9: Backscattered electron image of the dirty gypsum produced at stage B in Figure 4.7.

As it was stated before it is logical to expect that the addition of calcium hydroxide will produce a combination of insoluble calcium sulfate and calcium arsenate salts. An EDS mapping was done to the same region shown in Figure 4.8 and Figure 4.9. Results are shown in Figure 4.10.

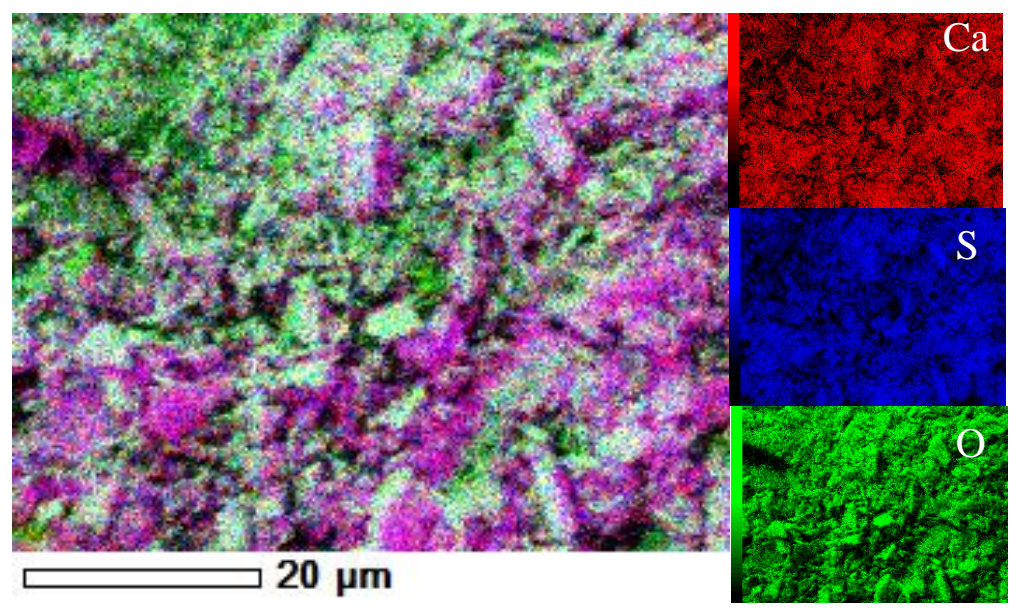

Figure 4.10: EDS map of Figure 4.9 with $\mathrm{Cu}$ (red), $\mathrm{S}$ (blue) and $\mathrm{O}$ (green).

The overlay picture of the three colours shows pink zones which indicate the presence of $\mathrm{Ca}$ and $\mathrm{S}$. White colouration on the other hand shows the presence of $\mathrm{Ca}, \mathrm{S}$ and $\mathrm{O}$ which is related to calcium sulfate or calcium hydrogen sulfate. As the lightest element the amount of oxygen might have been underestimated due to shielding effects by 
calcium and sulfur. Green zones show no relation between oxygen and the other mapped elements. These may be related to other oxygen containing species such as arsenate or hydrogen arsenate.
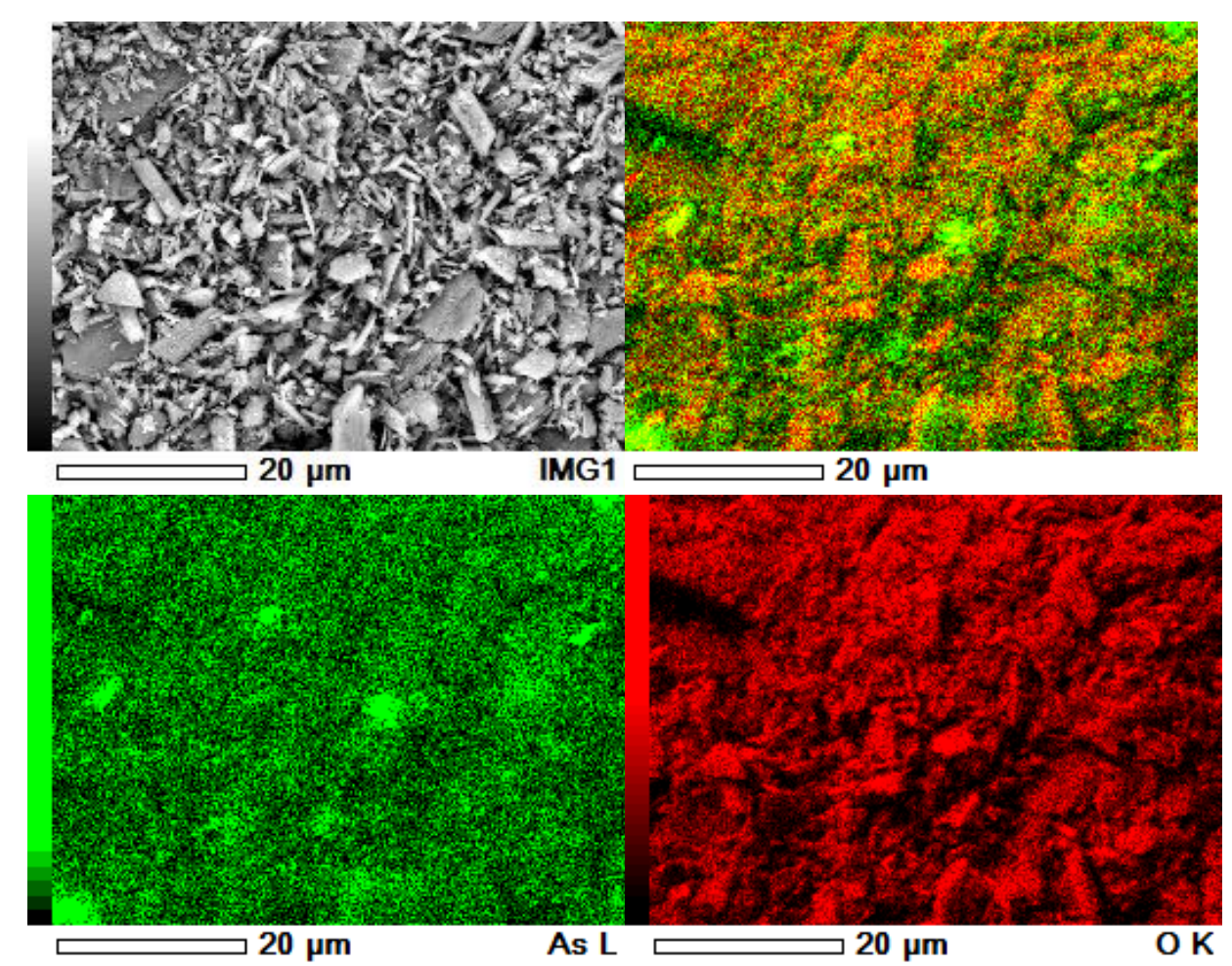

Figure 4.11: EDS map of Figure 4.9 for As (green) and O (red).

The same region was mapped for arsenic and oxygen to see there relation on the surface. The overlay image in Figure 4.11 shows that there is a correlation between the occurrences of these two elements on the surface of the sample being, up to a certain extent, evenly distributed throughout the sample surface. Nonetheless, there are few but intense green spots indicating arsenic rich species being present. 


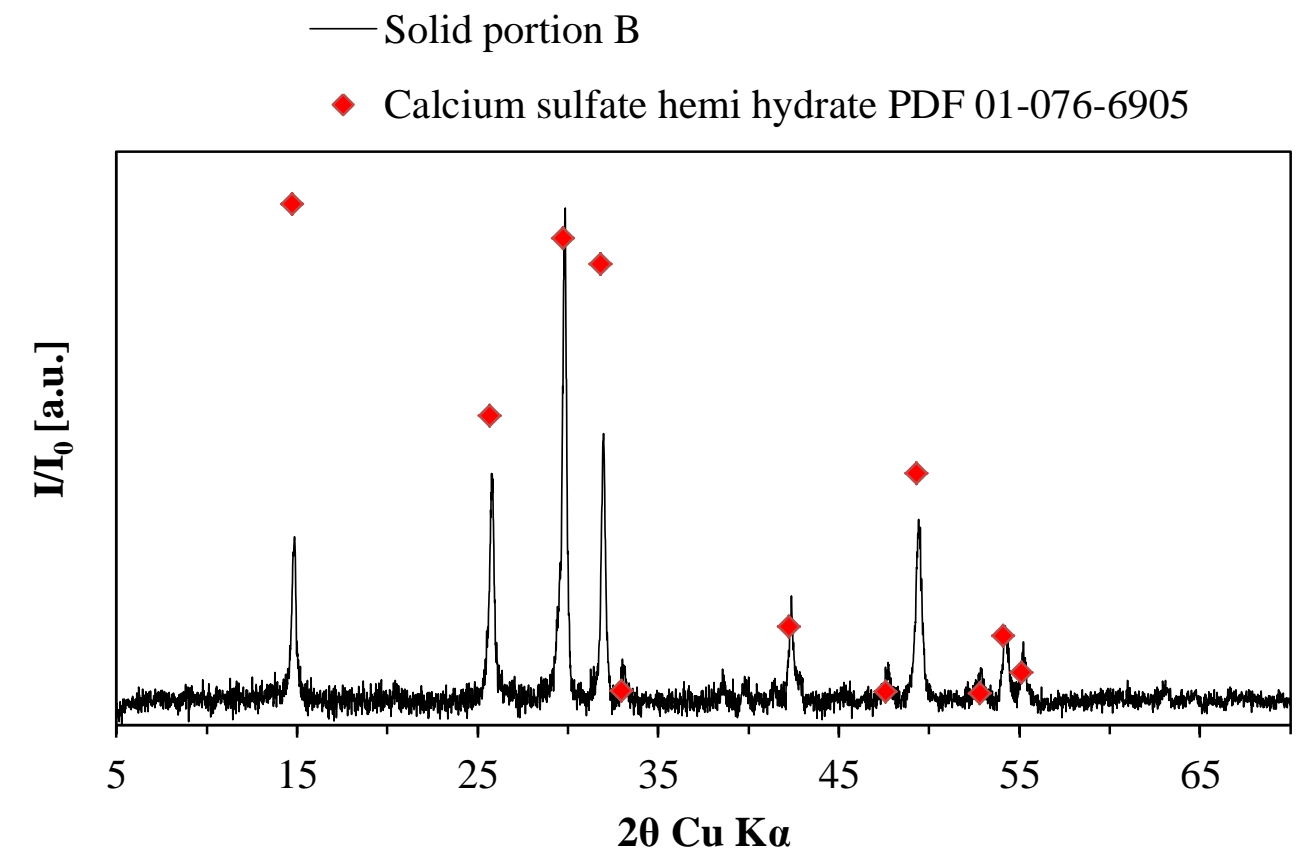

Figure 4.12: XRD pattern corresponding to solid fraction B in Figure 4.7.

Further information was gathered by grinding the sample and analysing it using XRD. The peaks scored 74 for $\mathrm{Ca}\left(\mathrm{SO}_{4}\right)_{2} \cdot 1 / 2 \mathrm{H}_{2} \mathrm{O}$ (PDF number 01-076-6905). The hemihydrate form may be due to the dehydration of calcium sulfate dihydrate during the preparation of the sample. This finding is consistent to the EDS mapping for this sample, although no diffraction peak associated to arsenic containing crystal could be found.

Following the diagram shown in Figure 4.7, after filtering the solid portion B, NCaSil was added to liquid A. This solution contained a concentration of copper of $181 \mathrm{mg} \mathrm{L}^{-1}$ since it was diluted by the addition of lime. Half of the initial amount of $\mathrm{Pb}$ and $\mathrm{Fe}$ remained in solution. $18.7 \%$ of the initial amount of arsenate was still in solution A. The addition of NCaSil resulted in the formation of a solid phase and a new liquid phase. A kinetic study of the uptake of copper, lead and iron using solution A was carried out and the results are presented in Figure 4.13. The three metals were effectively removed from solution $(>99 \%)$. The reaction between $\mathrm{NCaSil}$ and copper ions was extremely fast, taking only 3 minutes to reduce the concentration of copper from 181 to $1.36 \mathrm{mg} \mathrm{L}^{-1}$. 


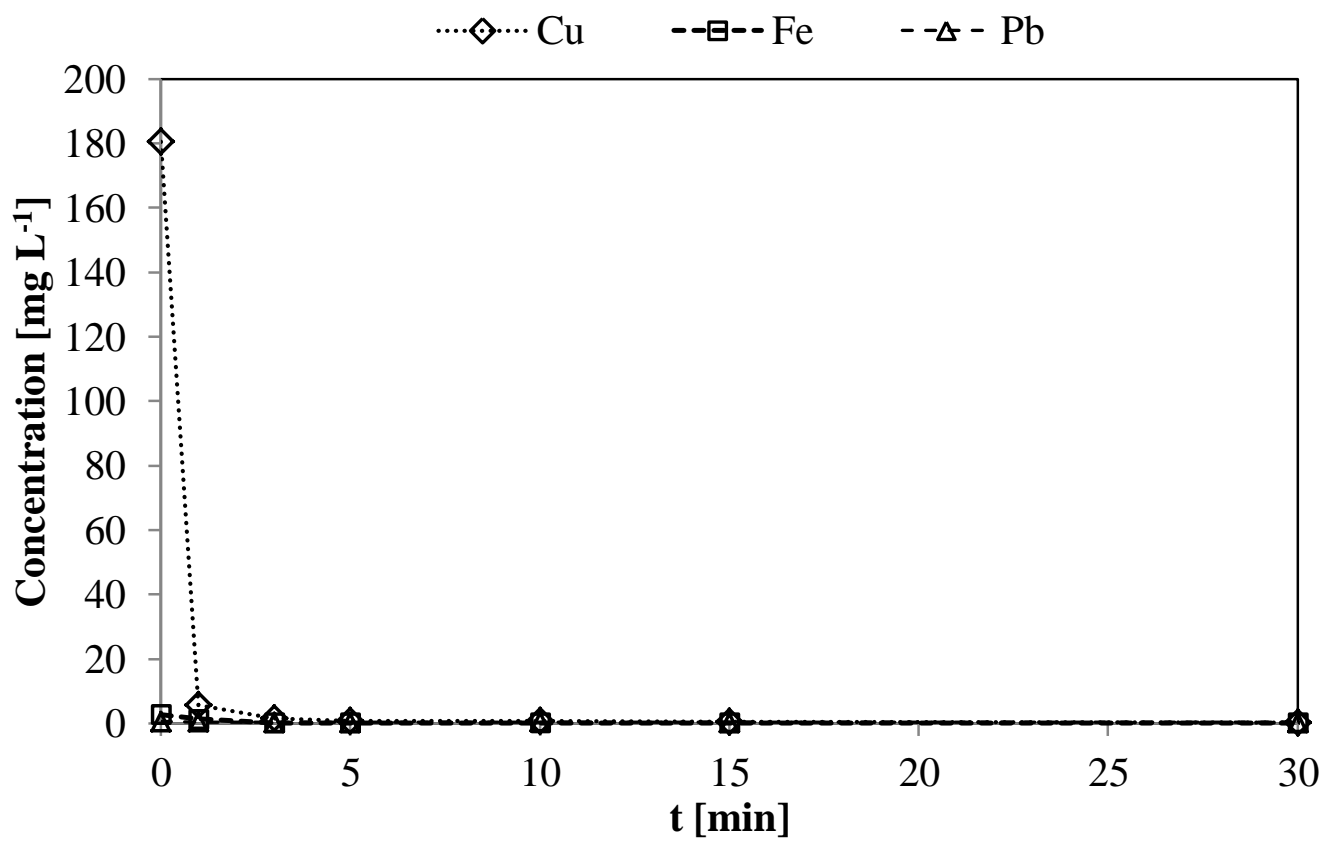

Figure 4.13: Kinetics of copper uptake by NCaSil from solution A in Figure 4.7.

The concentration of copper, lead and iron in solution D was below the detection limit. The $\mathrm{pH}$ of the solution was raised from 3 to 4 by the addition of NCaSil. In the case of arsenate and molybdenum, $97 \%$ and $64 \%$ of the initial amount in solution remained in solution, respectively. The amount of calcium in solution D is $13 \%$ higher than solution A due to $\mathrm{Ca}^{2+}$ leaching from NCaSil. The resulting solid $\mathrm{C}$ is rich in copper with low content of arsenate and sulfate as can be seen in Figure 4.7. This is an excellent result since even under extremely high concentrations of arsenate in solution and an adsorbent rich in calcium ions, only a small amount of arsenate precipitates. This would help in the real life operation to produce a copper rich solid with minor amount of impurities. NCaSil also exhibits a slight affinity towards removing molybdenum from solution, removing $36 \%$ of the amount present in solution A. The XRD pattern for solid portion $\mathrm{C}$ is shown in Figure 4.14. 


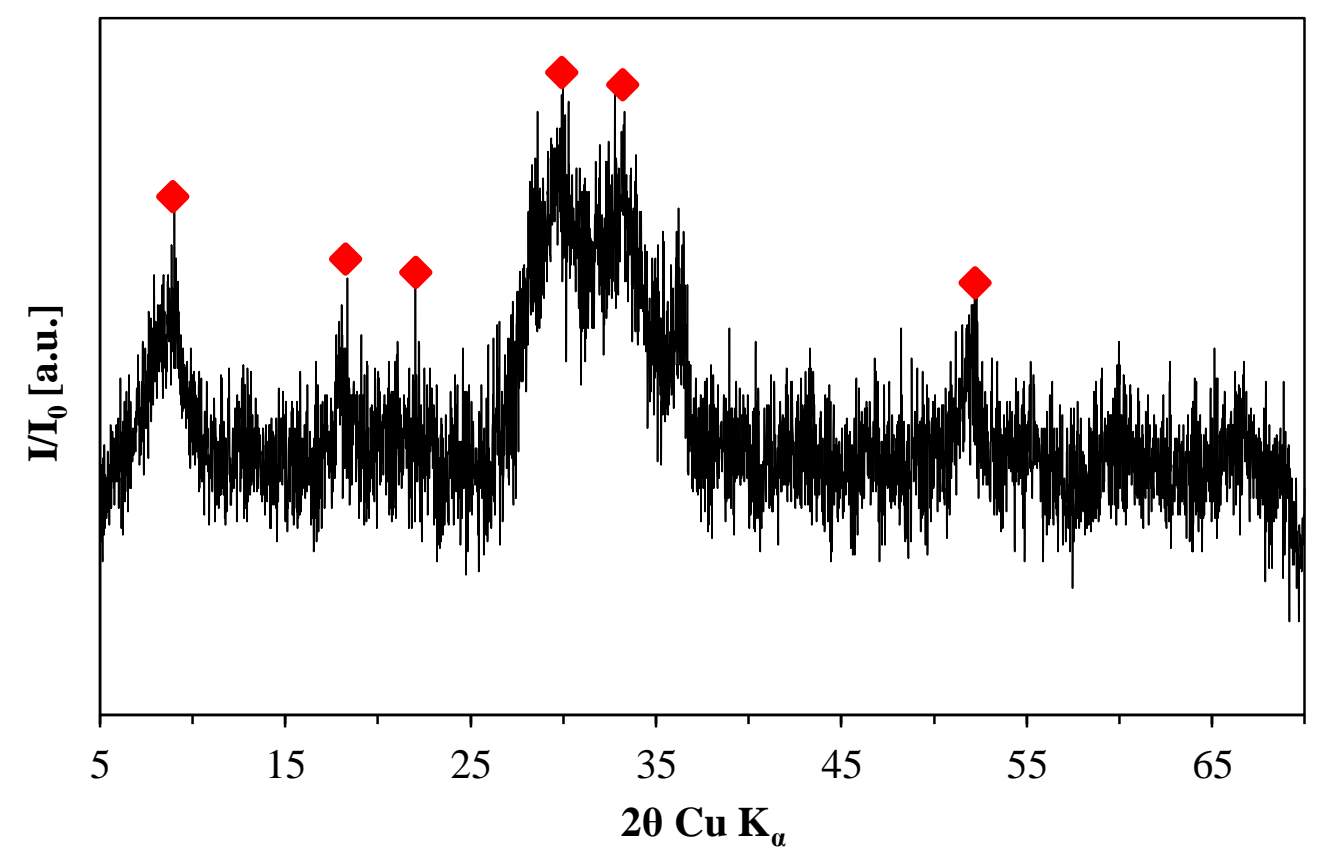

Figure 4.14: XRD pattern corresponding to letter $C$ in Figure 4.7.

Although peaks did not match to any of the crystal structures in the database, peaks at $9.0^{\circ}, 18.3^{\circ}, 22.0^{\circ}, 29.9^{\circ}, 32.8^{\circ}$ and $51.9^{\circ} 2 \theta \mathrm{Cu} \mathrm{K}$ could be associated to poorly crystalline gypsum, NCaSil or posnjakite $\left(\mathrm{Cu}_{4}(\mathrm{OH})_{6} \mathrm{SO}_{4}\right)$. Calcium carbonate can be discarded due to a $\mathrm{pH}$ value of 4 in solution D. Copper might be forming posnjakite as it was shown in Figure 3.32 at initial concentrations of copper near $2 \mathrm{mmol} \mathrm{L}^{-1}$ this mineral may develop. Although the final $\mathrm{pH}$ differs between these two samples, the results shown in Table 3.5 has a final $\mathrm{pH}$ value in the solution near 8 and the emulated mining waste had a value of 4 . Therefore, the amount of $\mathrm{OH}^{-}$groups available to form the crystal is larger in the former. 


\subsection{Chapter Conclusions}

Weak acid is generated in two different plants during the cleaning and cooling of furnace gases using water scrubbers at the sulfuric acid plant of the El Teniente mine in Chile. The weak acid is composed of a liquid (supernatant) and solid phase (suspended solids). The supernatant contains a high concentration of total arsenic of $6.0 \pm 0.8 \mathrm{~g} \mathrm{~L}^{-1}$ for Plant 1 and $6.5 \pm 1.3 \mathrm{~g} \mathrm{~L}^{-1}$ for Plant 2. Sulfate concentrations over the studied period of time were $43 \pm 10 \mathrm{~g} \mathrm{~L}^{-1}$ for Plant 1 and $69 \pm 12 \mathrm{~g} \mathrm{~L}^{-1}$ for Plant 2. The higher concentration of arsenic and lower concentration of sulfate compared to those found in other smelters could be related to the lower metal sulfide and higher arsenic content in the ore being processed at El Teniente. Suspended solid concentrations showed large spikes on a few dates, which contributed to a large value for the standard deviation for the solids concentrations. Solid concentrations of $0.23 \pm 0.63 \%$ wt for Plant 1 and $0.97 \pm 0.95 \%$ wt for Plant 2 were reported for the period from April to October 2010 . Overall, both plants operated almost all of the time within the control limits and exhibited very few concentration spikes over the studied period of time.

Chemical analysis of the supernatant showed that it had a low $\mathrm{pH}$-value of 0.45 and contained a high concentration of heavy metals. Copper had the highest concentration with a value $562 \mathrm{mg} \mathrm{L}^{-1}$. Taking in consideration the concentration of the different analytes the weak acid can be classified as an extremely acidic and high metal content waste.

Even though As and sulfate concentrations differ from those reported for other furnaces around the world, the measured parameters, such as arsenic, copper and sulfate, are still of the same order of magnitude as those described in the literature, for example those reported for the Toyo smelter in Japan.

High concentrations of copper in solution represent an estimated loss of 100 tons of this metal per year. A process such as the one utilized in Toyo's smelter in Japan, where copper ions are precipitated by the addition of NaHS should be considered.

SEM, EDS, powder-XRD and ICP-OES analysis of the suspended solids found in weak acid waste showed that this phase was mainly composed of sub-micron particles of anglesite $\left(\mathrm{PbSO}_{4}\right)$. This portion of the studied waste should be carefully investigated in future research to assess potential threats posed by these sub-micron particles and to suggest measures for preventing their release into the environment. 
The treatment of the emulated weak acid with $\mathrm{NCaSil}$ is feasible at $\mathrm{pH}$ values of the weak acid above 3 and below 5. This prevents the rapid loss of structure of NCaSil which in consequence allows the adsorption of copper ions onto the surface. Additionally it also prevents removal copper ions during the $\mathrm{pH}$ adjustment stage as $\mathrm{Cu}(\mathrm{OH})_{2}$ which is readily formed at $\mathrm{pH}$ above 5 in solution.

The uptake of $\mathrm{Cu}^{2+}$ onto NCaSil was extremely fast reaching equilibrium in only 5 minutes. The resulting solid had a low amount of arsenate and sulfate content demonstrating that the adsorption of copper ions is preferential making it suitable for industrial applications.

This process could be coupled to the one proposed by BAMAG $\mathrm{GmbH}$ which will actually optimize the utilization of this waste to produce two marketable products such as clean gypsum and a copper-rich calcium silicate. The remnant solution D may continue to the normal treatment of this waste that consists of precipitating arsenic containing species and disposal in a landfill. 


\section{Chapter 5 , Study of the continuous uptake of $\mathrm{Cu}^{2+}$ by NCaSil}

This chapter describes attempts to find a column-type design that would allow operation of a water purification system based on NCaSil. A comparison between a conventional axial flow column and a radial flow column as an improvement over previous continuous adsorption systems with $\mathrm{NCaSil}$ is analysed.

\subsection{Introduction to packed column sorption}

The use of packed columns in water treatment is wide spread [6, 114]. Ion exchange resins are used in packed columns as softeners and for the deionization of water in order to prevent scaling. The use of columns makes it possible to have a steady state of production of water over a period of time. Water usually flows downward in a column, driven either by pressure or gravity. It is common to find that adsorbents can be regenerated, increasing the life cycle of the process.

Usually the amount of liquid flowing inside the column is expressed as hydraulic loading $(H)$ and it is defined as the flow rate of a fluid passing through a known cross sectional area of empty bed. Typical operating design parameters for activated carbon packed columns are [114]: 1) fluid residence times of 15 to 35 min (longer residence times generally produce a greater degree of contaminant removal and are closely related to the kinetic and thermodynamic of the adsorption), 2) $H$ values of $0.1-0.2 \mathrm{~m}^{3} \mathrm{~min}^{-1} \mathrm{~m}^{-2}$ for downward and 0.2 to $0.4 \mathrm{~m}^{3} \mathrm{~min}^{-1} \mathrm{~m}^{-2}$ for upward flow 3 ) depths ranging from 3 to 12 metres, with 3.5 to 6 metres being typical, and 4) 
pressure drops across the column of less than $22.3 \mathrm{kPa}$ per $\mathrm{m}$ of depth. Extra column space of 10 to $50 \%$ is usually provided if backwashing is planned to enable bed expansion.

The water is fed at a steady rate, and the contaminant is adsorbed producing contaminant-free water. However, the adsorbent particles start to saturate with the contaminant, from the inlet towards the outlet end, until at some point a "breakthrough" of contaminant occurs in the outlet water stream. The plot of the concentration of the contaminant as a function of the time or effluent volume is called the breakthrough curve. In Figure 5.1 is shown the progress of the sorption zone in the column and its corresponding breakthrough curve.

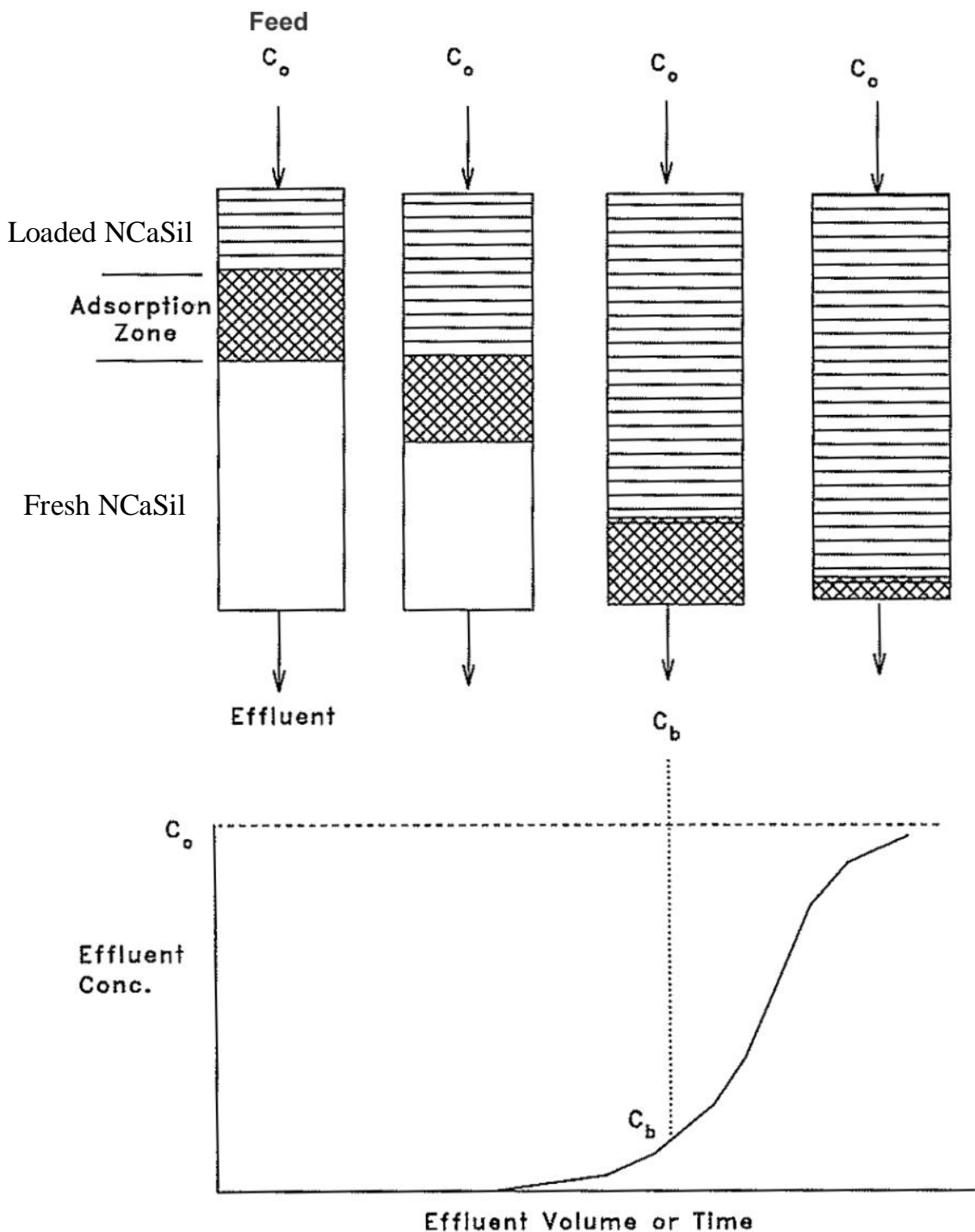

Figure 5.1, Progression of the sorption zone through a conventional axial flow packed column taken from reference [114].

When the outlet concentration reaches an undesirable value $\left(C_{b}\right)$, the operation is stopped. It is common that activated carbon columns are in parallel so when the 
outlet of column 1 reaches $C_{b}$, the inlet of column 1 is diverged to column 2 and the carbon from column 1 is disposed and replaced. Afterwards, the inlet is connected to the outlet of column 2. This way it is possible to assure a continuous process.

\subsubsection{Column operational parameters}

As previously discussed, were the standard design of a column consists of a cylinder with the water flowing through the cross-sectional area which is defined as:

$$
A=\frac{\pi}{4} D^{2}
$$

where $A$ is the cross sectional area in $\mathrm{m}^{2}$ and $D$ is the column diameter in $\mathrm{m}$. The flow through the cross-sectional area of the column is defined as,

$$
Q=A \cdot v \cdot \varepsilon
$$

where $Q$ is the flow in $\mathrm{m}^{3} \mathrm{~min}^{-1}, A$ is the area through which the fluid flows in $\mathrm{m}^{2}, v$ is the average axial velocity of the fluid in $\mathrm{m} \mathrm{min}^{-1}$, and $\varepsilon$ is the column free space between the particles (void fraction). The interstitial velocity is $\varepsilon \cdot v$ in $\mathrm{m} \mathrm{min}^{-1}$. In the engineering literature the amount of liquid passing through a cross-sectional area in a column is often referred, as previously mentioned (Section 5.1), as hydraulic loading $(H)$ given in $\mathrm{m}^{3}$ of fluid per minute per $\mathrm{m}^{2}$ of cross-sectional area. Even though $H$ and $\varepsilon \cdot v$ have the same numerical values, their meaning differs since their units are different. Engineers often use the term superficial velocity $\left(U_{0}\right)$ to denote the velocity of the fluid when the column is empty $(\varepsilon=1)$. On the other hand, if the void fraction of the column $(\varepsilon)$ is 0.4 , the superficial velocity will be $40 \%$ the actual interstitial velocity.

In chemical engineering literature there are several dimensionless numbers that help to understand the behaviour of a process. The Reynolds number $(\operatorname{Re})$ gives a measure of the ratio of the inertial forces to the viscous forces and is defined by eq. 5.3. The value of this number gives the turbulence of a fluid transported through an empty cross-sectional area.

$$
R e=\frac{v D \rho}{\mu}
$$

where $v$ is the average velocity in $\mathrm{m} \mathrm{min}^{-1}, D$ is the diameter of the cross sectional area in $\mathrm{m}, \rho$ is the fluid's density in $\mathrm{kg} \mathrm{m}^{-3}$, and $\mu$ is the kinematic viscosity in $\mathrm{kg}$ $\min ^{-1} \mathrm{~m}^{-1}$. When the value of the Reynolds number is between $0<R e<2100$ the fluid has a laminar flow, when values are $2100<R e<4200$ the fluid has a 
transitional flow, when the Reynolds number is bigger than 4200 the fluid has a turbulent flow.

In the case of packed columns the Reynolds number is modified to account for the void fraction and particle shape of the adsorbent material. The modified Reynolds number is shown in Eq. 5.4.

$$
N_{\mathrm{Re}^{\prime}}=\frac{D_{p} G}{\mu}
$$

Where $G$ is the superficial mass velocity based on the empty chamber cross section in $\mathrm{kg} \mathrm{s}^{-1} \mathrm{~m}^{2}, \mu$ is the fluid viscosity in $\mathrm{kg} \mathrm{s}^{-1} \mathrm{~m}^{-1}$ and $D_{p}$ is the particle diameter when the packing material is spherical. When the particle is not spherical this parameter depends on several factors as shown in equation 5.5.

$$
D_{p}=\frac{6(1-\varepsilon)}{\phi_{s} S}
$$

Where $S$ is the area of particle surface per volume of bed and is equal to $S_{0}(1-\varepsilon)$ in $\mathrm{m}^{2} \mathrm{~m}^{-3} ; S_{0}=$ surface of the particle per unit of volume of solids in $\mathrm{m}^{2} \mathrm{~m}^{-3}, \varepsilon$ is the void space inside the packed column (free space between particles of adsorbent) and $\phi_{s}$ is the shape factor that may be obtained from appendix A-20 for different materials. The fluid is considered to be at turbulent regime when $N_{R e^{\prime}}>100$.

The Reynolds number is an important parameter because during a continuous adsorption process laminar flows increase radial dispersion inside the column. While turbulent flows help to reduce the static liquid film thickness surrounding the particle, thus facilitating the adsorption by reducing the resistance of the mass transfer from the liquid to the solid the phase. Nonetheless, increasing the flow will result in early breakthrough of the contaminant, due to a decrease in the residence time inside the column, and a significant increase in the pressure drop through the column.

\subsubsection{Pressure drop}

The dependence on the pressure drop for an incompressible fluid passing through a bed of granular solids with the flow, friction, bed length, particle diameter, particle shape, void fraction and density was studied by Leva in 1949 [115]. The mathematical expression derived from his study is presented in equation 5.6. 


$$
\Delta p=\frac{2 f_{m} U_{0}^{2} L(1-\varepsilon)^{3-n}}{D_{p} \rho \phi_{s}^{3-n} \varepsilon^{3}}
$$

Where $\Delta \mathrm{p}$ is the pressure drop in $\mathrm{Pa}, f_{m}$ is the friction factor and is obtained from appendix A-21 (dimensionless), $U_{0}$ is the fluid superficial mass velocity based on an empty chamber cross section in $\mathrm{kg} \mathrm{s}^{-1} \mathrm{~m}^{-2},{ }^{5} L$ is the bed length, $\varepsilon$ is the void fraction inside the column (from 0 to 1 ), $n$ is an exponential that can be calculated from appendix, $\rho$ is the fluid density and $\phi_{s}$ is the shape factor of the packing material.

It is noteworthy that the pressure drop inside a packed column is linearly dependant on the bed length; inversely proportional to particle diameter; increasing the flow will result in a square increase of the pressure drop; reducing the void fraction will have an enormous impact due the high value of the exponential associated to this variable; and the shape factor also strongly influences the pressure drop due to the exponential associated with it. If all variables are kept constant the pressure drop is constant over time.

The influence of the void fraction and the shape factor becomes less predominant at high Reynolds number as shown in appendix A-21. This dependence is clearly noticeable for values of $N_{R e}$, near $10^{4}$ are used due to the fact that $n$ approaches a value of 2 which results in the exponent value of $(1-\varepsilon)$ and $\phi_{s}$ equal to 1 . Nevertheless, increasing $N_{R e}$, will also generate pressure loss and early breakthroughs due to an increase in the flow. Undoubtedly, there is a complex relation between key operational parameters and the pressure drop. Hence special care should be taken in order to find the most suitable values that allow operating a column in a practical way.

Large pressure drops have been observed during the preparation stage of NCaSil, where long times are required to filter $\mathrm{NCaSil}$ from solution. Nonetheless this variable has never been quantified nor reported. Understanding how pressure drop is influenced is important because this variable could give an insight into the sorption of $\mathrm{Cu}^{2+}$ in a continuous regime. In fact, this was the reason Borrmann and Fonseca [116] studied an alternative approach towards the continuous use of NCaSil for the uptake of metal ions that would lessen the pressure drop.

\subsubsection{Special considerations in regards to radial flow column}

Radial flow columns (RFCs) are mainly used in bioseparations of proteins and organic compounds [50-52]. These columns offer higher production rates, low 
pressure drops, ease of packing, less footprint and better linear scalability than conventional axial flow columns [117]. In the case of chromatographic applications they offer narrower peaks due to less axial dispersion. However, manufacturers usually do not mention the most important drawback of this type of column, which is the reduction of the number of theoretical plates compared to conventional axial flow columns due to the shortening of the bed length. Nonetheless, this usually is not an issue if the chemical structure of the compounds is sufficiently different and the correct choice of solvent and packing material is made.

It is noteworthy that the author was unable to find literature regarding the use of RFC in the uptake of metal ions from solution. The literature available on RFCs is focused on chromatographic separations.

From the operational point of view, it is important to note that the cross sectional area of the radial flow column varies as the fluid flows through the adsorbent bed. In the specific case of this thesis an outward flow was chosen, therefore the cross sectional area increases when the fluid travels through the adsorbent bed. Based on this an average cross sectional area by which the fluid flows through has to be considered. In a similar manner to tubular heat exchanger calculations [115], one may calculate the average logarithmical mean area of the mantle of the cylinder through which the flux occur using equation 5.7.

$$
A_{\ln }=\frac{A_{2}-A_{1}}{\ln \left(\frac{A_{2}}{A_{1}}\right)}
$$

Where $A_{\ln }$ is the mean logarithmic area, $A_{l}$ is the area of the cylinder mantle of the inner feed cylinder and $A_{2}$ is the area of the cylinder mantle of the sorbent holder. $A_{\ln }$ has to be used instead of $A$ in all equations related to RFC operation. 


\subsubsection{Borrmann and Fonseca approach to the continuous uptake of $\mathrm{Cu}^{2+}$}

Two prior studies have utilised NCaSil in a continuous uptake system. The first one was done by Cairns in a fluidised bed reactor for the uptake of phosphate ions [37]. The second was carried out Fonseca [116] using the design shown in Figure 5.2. This study will focus on the latter since it is related to copper adsorption. The RFC design presented in this study is intended to improve Bormann and Fonseca's approach.

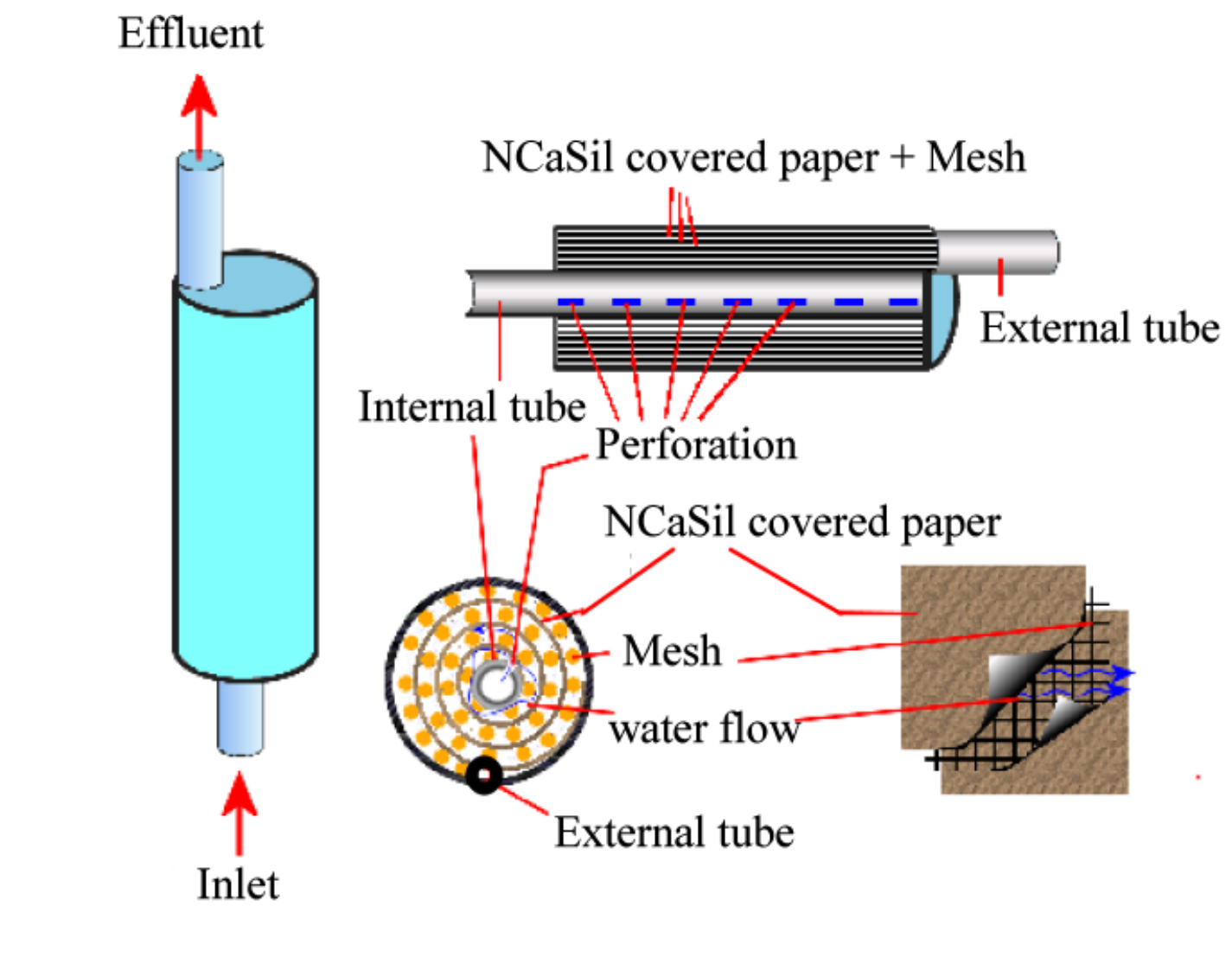

Figure 5.2: Borrmann design for the treatment of aqueous solutions containing high concentrations of metal ions. The experiment was carried out by Fonseca. The image is a reprint from reference [116]. The dimensions of the cartridge are: internal diameter $3 \mathrm{~cm}$; external diameter $5 \mathrm{~cm}$; length of the chamber where the paper is placed $9 \mathrm{~cm}$.

In Figure 5.2 a schematic of the Borrmann design is shown which consists of a plastic housing containing a centre tube with perforations as the inlet. Paper coated with NCaSil is wrapped around this tube. Water flows from the perforations outwards contacting the solution with the material on the papers' surface, allowing the selected contaminant to reach the surface of the adsorbent.

Fonseca did an exploratory experiment where she coated paper with a mixture of $\mathrm{NCaSil} /$ Latex or NCaSil/starch which resulted in only a small amount of the initial copper ions removed from solution. Results of such experiment are presented in Figure 5.3. 


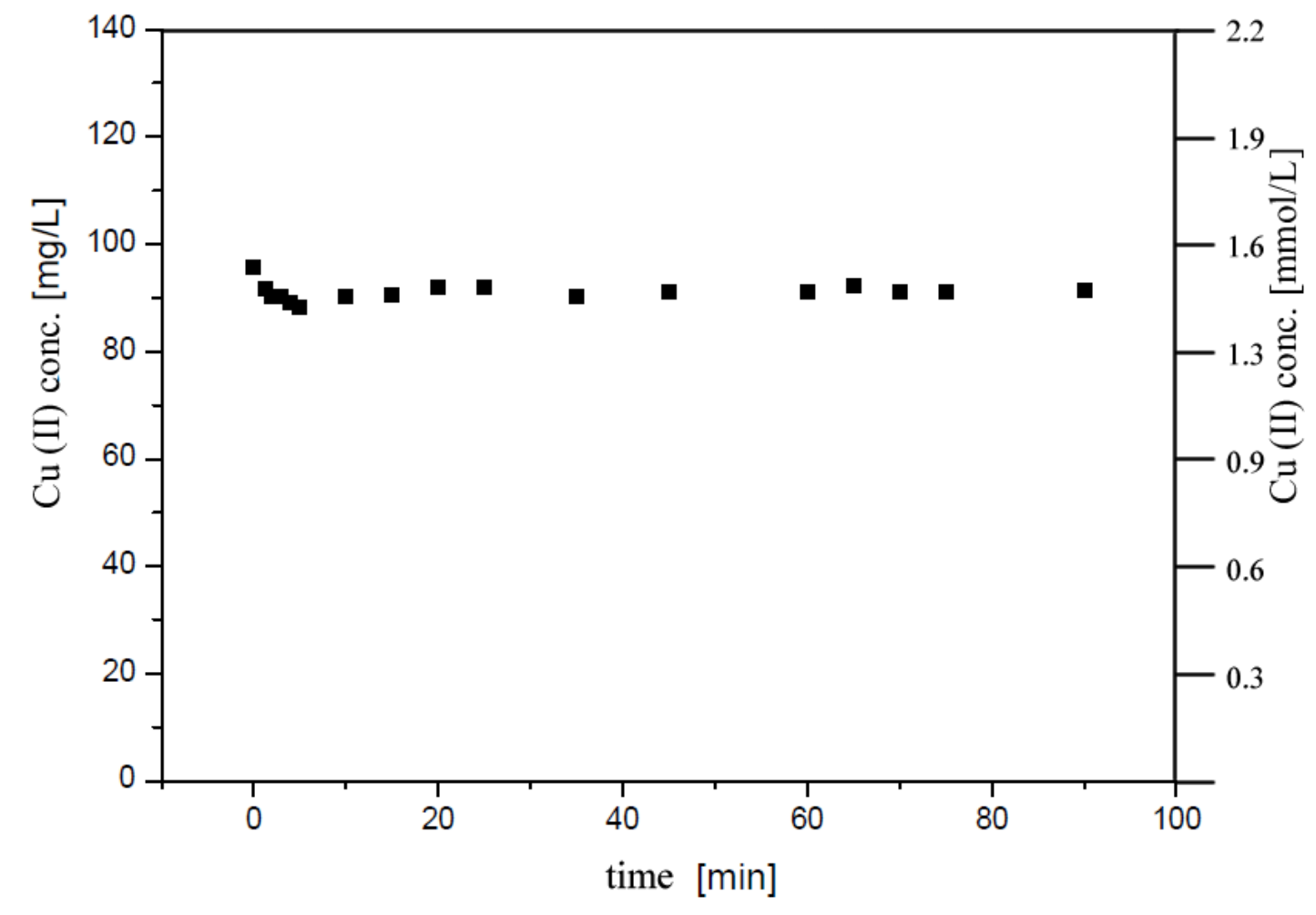

Figure 5.3: Continuous uptake of copper carried out by Fonseca using the design shown Figure 5.2. Experimental conditions: $\left[\mathrm{Cu}^{2+}\right] 1.6 \mathrm{mmol} \mathrm{L}^{-1}$ using copper chloride; flow $14.5 \mathrm{ml} \mathrm{min}^{-1}$; Hydraulic residence time $1.3 \mathrm{~min}$; temperature of the feed solution $303 \mathrm{~K}$. This figure was translated to English from the original version in reference [116].

Figure 5.3 shows that only a small fraction of the copper added into solution is removed. The initial adsorption rate of $\mathrm{Cu}^{2+}$ uptake onto NCaSil in batch is in the order of $1 \mathrm{mmol} \mathrm{g} \mathrm{m^{-1 }}$ at this temperature as shown in Table 3.2 in Chapter 3. In Fonseca's study, $23 \mu \mathrm{mol}$ of $\mathrm{Cu}^{2+} \min ^{-1}$ were pumped through this device, ${ }^{6}$ therefore most $\mathrm{Cu}^{2+}$ present should be removed from solution at least during the first minutes at this concentration. The reason why there is no sigmoidal behaviour of the experimental is due to certain flaws in the design. The Borrmann-Fonseca design is not sealed at the end of each sheet of paper and the cartridge wall. Hence, once the void space within sheet of paper is full of liquid, the fluid will travel the path that offers the least resistance by-passing the paper-loaded NCaSil. Fonseca didn't comment about the design drawback. Any future design should ensure that the solution becomes in contact with the solid before leaving the device. Nonetheless, Fonseca tried to improve the performance by increasing the number of paper sheets inside to a total number of three, which increased the amount of NCaSil proportionally. Results of this second experiment are shown in Figure 5.4.

${ }^{6}\left[\mathrm{Cu}^{2+}\right] \times \mathrm{Q}=1.6 \mathrm{mmol} \mathrm{L}^{-1} \times 14.5 \mathrm{ml} \mathrm{min}{ }^{-1}=0.0232 \mathrm{mmol} \mathrm{min}^{-1}=23.2 \mu \mathrm{mol} \mathrm{min}{ }^{-1}$ 


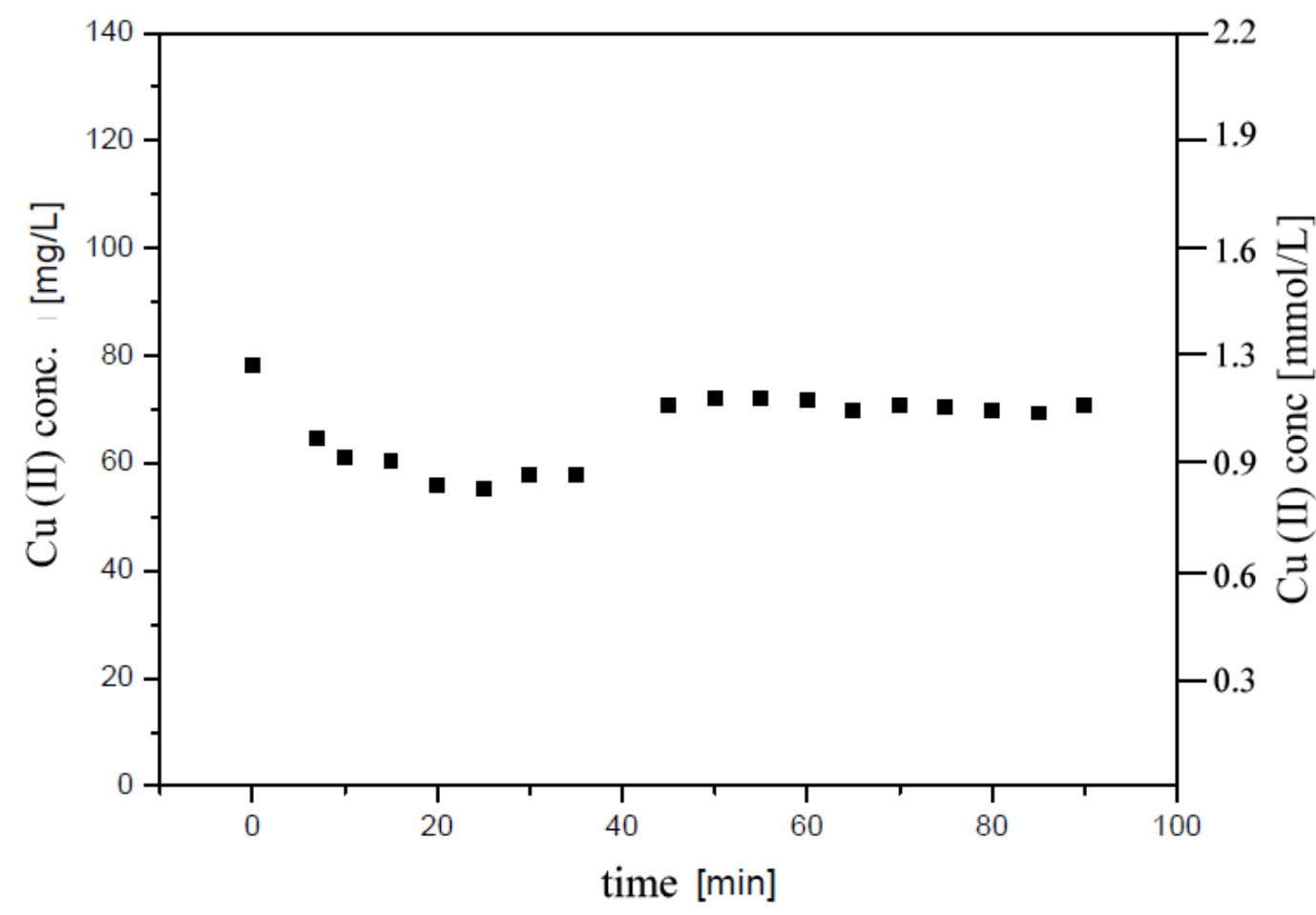

Figure 5.4: Continuous uptake of copper carried out by Fonseca using the design shown Figure 5.2. Experimental conditions: $\left[\mathrm{Cu}^{2+}\right] 1.6 \mathrm{mmol} \mathrm{\textrm {L } ^ { - 1 }}$ using copper chloride; flow $14.5 \mathrm{ml} \mathrm{min}{ }^{-1}$; Hydraulic residence time $1.3 \mathrm{~min}$; temperature of the feed solution $303 \mathrm{~K}$. This figure was translated to English from the original version in reference [116].

Experimental data shown in Figure 5.4 follows the same trend observed in Figure 5.3, but this time is more pronounced. Increasing the amount of NCaSil inside the device produces better $\mathrm{Cu}^{2+}$ removal from solution as expected, but still is not good enough to proceed to a scale up of the process. The trend is far from the desired efficiency which should follow the sigmoidal behaviour shown in Figure 5.1, where for a long period of time the targeted contaminant is removed from solution with only low or undetectable concentrations remaining in the effluent.

These results led to the idea of using a radial flow column (RFC) which forces the solution to contact NCaSil before the fluid can leave the column, and offers all the advantages previously discussed. 


\subsection{Results and discussion for the continuous uptake of $\mathrm{Cu}^{2+}$ using NCaSil}

At the beginning of this study there was no reported result regarding NCaSil inability to operate inside a packed column, except for a few comments from Borrmann who reported that NCaSil will produce high pressure drops. In fact the pressure drop was one of the main reasons which led Borrmann to seek an alternative for conventional axial flow columns, which in turn led to the design shown in Figure 5.2 used by Fonseca in her thesis [116].

Therefore there was a need to record the magnitude of the pressure drop over time and its dependence on flow and bed height. These experiments will give an insight into the correct ratio between column heights to cross-sectional area and the best operating conditions, such as flow and feed concentration of the contaminant before starting to work with the RFC.

Following the studies reported earlier in Chapter 3 (sections 3.2.4, 3.2.5, 3.4.3, and 3.4.4) it was expected that the main issue with NCaSil is that once it is contacted with an aqueous solution, its morphology changes due to leaching of $\mathrm{Ca}^{2+}, \mathrm{OH}^{-}$and amorphous silica in the form of monomers and oligomers. This will lead to a collapse of the structure and generate changes in the pressure drop.

In order to prevent the structure from collapsing rapidly, experiments where the column was packed with fresh wet NCaSil slurry instead of the dried ethanol washed NCaSil were tried. Although the operation of filling the column proved to be easier than with the powder, the slurry offered less void fraction for the fluid to flow through, generating elevated backpressures above $200 \mathrm{kPa}$, for $5 \mathrm{~cm}$ bed heights and a flow of $5 \mathrm{ml} \mathrm{min}{ }^{-1}$, when a $\mathrm{Cu}^{2+}$ solution was passed through a conventional axial flow column. It is noteworthy that most laboratory glassware is not meant to withstand $200 \mathrm{kPa}$ above atmospheric pressure. Hence, this restricts the use of NCaSil to the form of powder to pack columns. Nonetheless, using NCaSil in the form of a powder also experienced similar pressure limitations, which will be addressed later on in this section as they depended on specific experimental conditions

Overall, three different approaches were studied in order to understand the behaviour of NCaSil in continuous uptake arrangements. The first approach was a conventional axial flow column which attains the possibility of comparison with other results in 
the literature since this is the standard arrangement in lab scale experiments in the field. The second approach was a radial flow column (RFC), a novel approach to improve results obtained by Fonseca [116] in a previous attempt with a different design. Finally, the third semi-batch approach, consisted of immersing the RFC in a tank containing a $\mathrm{Cu}^{2+}$ solution and recirculating the fluid through the device.

\subsubsection{Continuous uptake of $\mathrm{Cu}^{2+}$ using a conventional axial flow column}

A column packed with NCaSil was studied as described in section 2.5.2, using different flows and concentrations. Bed heights above $5 \mathrm{~cm}$ could not be studied due to large pressure drops when solutions with $\left[\mathrm{Cu}^{2+}\right]>1.6 \mathrm{mmol} \mathrm{L}^{-1}$ were passed through the conventional axial flow column $(2 \mathrm{~cm}$ diameter $)$ with flows higher than

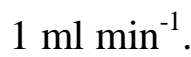

Keeping the flow and bed height constant and varying the concentration will help to understand how long it takes to reach breakthrough under different conditions, and project the scale up.

5.2.1.1 Experiments using a bed height of $2.5 \mathrm{~cm}$ and varying the concentration of $\mathrm{Cu}^{2+}$ in the feed solution

From Figure 5.5 to Figure 5.7 different concentrations of $\mathrm{Cu}^{2+}(1.6,7.9$ and $15.7 \mathrm{mmol} \mathrm{L}^{-1}$ ) were fed into the column keeping a constant flow at $5 \mathrm{ml} \mathrm{min}^{-1}$ and bed height of $2.5 \mathrm{~cm}$. 

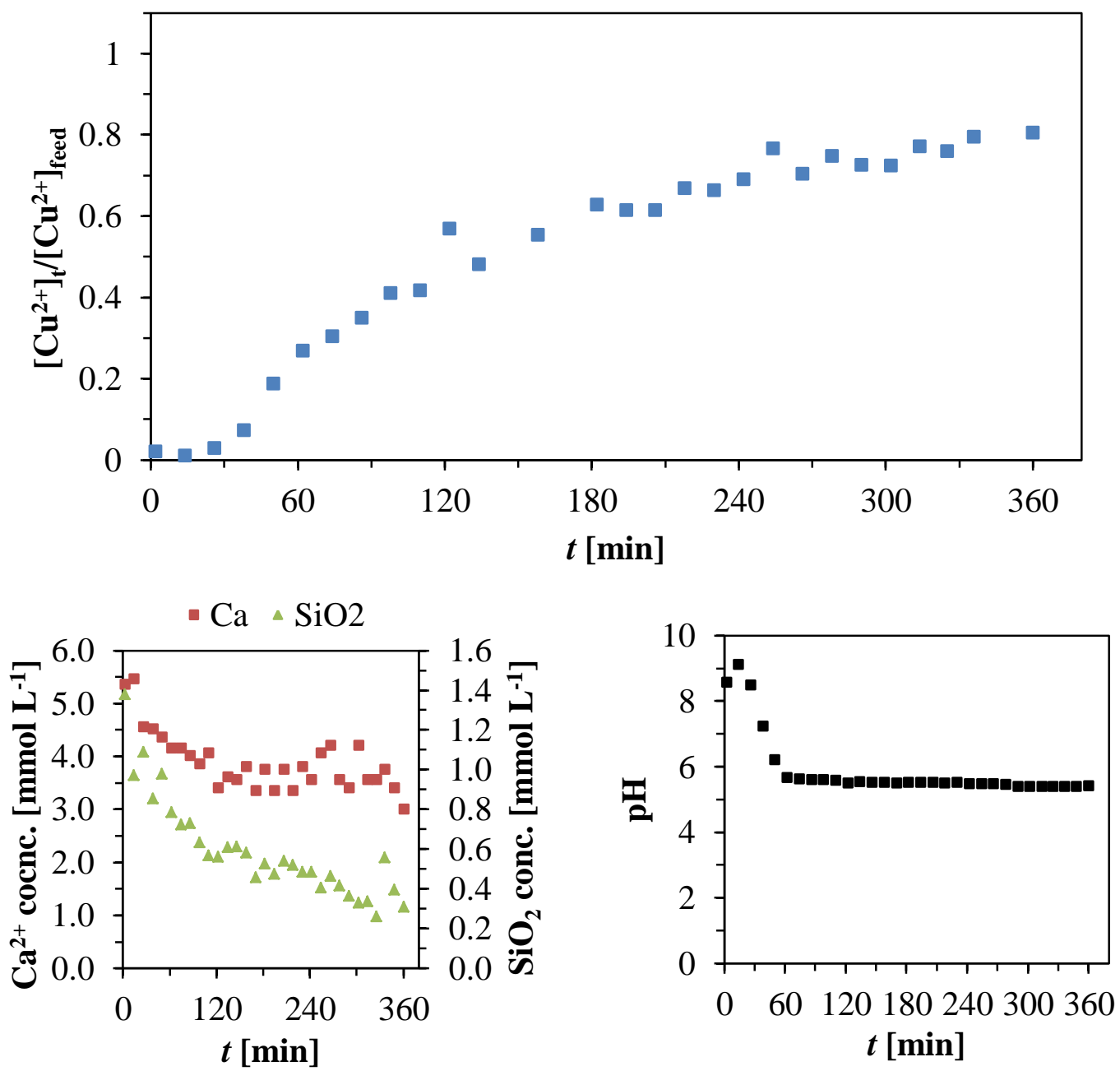

Figure 5.5: Conventional axial flow column experiment for the continuous adsorption of $\mathrm{Cu}^{2+}$ ions onto NCaSil. The graphs present the variation in the concentration of $\mathrm{Cu}^{2+}, \mathrm{Ca}^{2+}$ and monomeric silica as $\mathrm{SiO}_{2}$ in solution over time after it has passed through the column. The change of the pH over time is also shown. Experimental conditions: $\left[\mathrm{Cu}^{2+}\right] 1.6 \mathrm{mmol} \mathrm{L}{ }^{-1}$; bed height $2.5 \mathrm{~cm}$ equivalent to $500 \mathrm{mg}$ of NCaSil packed to bulk density; flow $5 \mathrm{ml} \mathrm{min}^{-1}$; temperature $293 \mathrm{~K}$. Experimental errors are detailed in Table 2.3.

Figure 5.5 shows that at concentrations near $1.6 \mathrm{mmol} \mathrm{L}^{-1}$ the column exhibited a high efficiency during the first $30 \mathrm{~min}$, removing $97 \%$ of the $\mathrm{Cu}^{2+}$ present in solution. The breakthrough occurred approximately after $30 \mathrm{~min}$ of starting the experiment. Then the concentration of $\mathrm{Cu}^{2+}$ in the outlet rose rapidly between 30 and 60 minutes. After $60 \mathrm{~min}$ it slowed down reaching a steady rate of growth until the end of the experiment. When the experiment concluded, the concentration of $\mathrm{Cu}^{2+}$ at the outlet was approximately $80 \%$ of the initial concentration, hence the column did not reach exhaustion. In order to reach exhaustion, times larger than $8 \mathrm{~h}$ may be needed. It was decided that the amount and quality of information would not be enhanced by reaching exhaustion $\left.\left(\mathrm{Cu}^{2+}\right]_{\mathrm{t}} /\left[\mathrm{Cu}^{2+}\right]_{\text {feed }}=1\right)$, and breakthrough times were sufficient to gain information for a rough scale-up design. 
The release of calcium and monomeric silica content appeared to follow a similar behaviour. A large amount of these two NCaSil constituents was released during the first 30 minutes with values greater than 4.5 and $0.8 \mathrm{mmol} \mathrm{L}^{-1}$ for $\mathrm{Ca}^{2+}$ and $\mathrm{SiO}_{2}$, respectively. The release of both components slowed down with time reaching concentrations of 3.0 and $0.3 \mathrm{mmol} \mathrm{L}^{-1}$ for calcium and monomeric silica, respectively. Furthermore neither $\mathrm{Ca}^{2+}$ nor $\mathrm{SiO}_{2}$ release reached exhaustion.

The trend of the variation of the $\mathrm{pH}$ value over time was closely related to the concentration of $\mathrm{Cu}^{2+}$ at the outlet of the column. The $\mathrm{pH}$ value remained above 8 during the first 30 minutes while copper remains below $3 \%$ of the initial concentration. Afterwards it decreased rapidly following the elution of $\mathrm{Cu}^{2+}$. The final $\mathrm{pH}$ value was closer to the original $\mathrm{pH}$ values of a solution containing $1.6 \mathrm{mmol} \mathrm{L}^{-1}$ of $\mathrm{Cu}^{2+}$ prepared from copper sulfate salt. The slight difference in values was mainly due to $\mathrm{OH}^{-}$still being present in solution.

During the experiment the pressure drop remained below $15 \mathrm{kPa}$ (not shown) without perceptible variations.

The time between breakthrough time and exhaustion time gives an idea of the length of the adsorption zone in Figure 5.1 also called mass transfer zone (MTZ). In this case the mass transfer zone appeared to be long as it does not resemble a sharp sigmoidal curve. Nevertheless, the low concentration of $\mathrm{Cu}^{2+}$ during the first 30 minutes indicates that the MTZ is less than $2.5 \mathrm{~cm}$. Changes on the morphology of NCaSil might induce length variation on the MTZ. At the beginning, where most of the uptake occurs, it offers a fresh surface that becomes saturated and passivated. Subsequently, the $\mathrm{pH}$ value of the solution became close to that of the feed solution. The presence of protons in solution may cause the hydrolysis of NCaSil resulting in the exposure of fresh surface to the solution. In parallel copper might also nucleate on the surface of crystals being formed as explained in Chapter 3. 

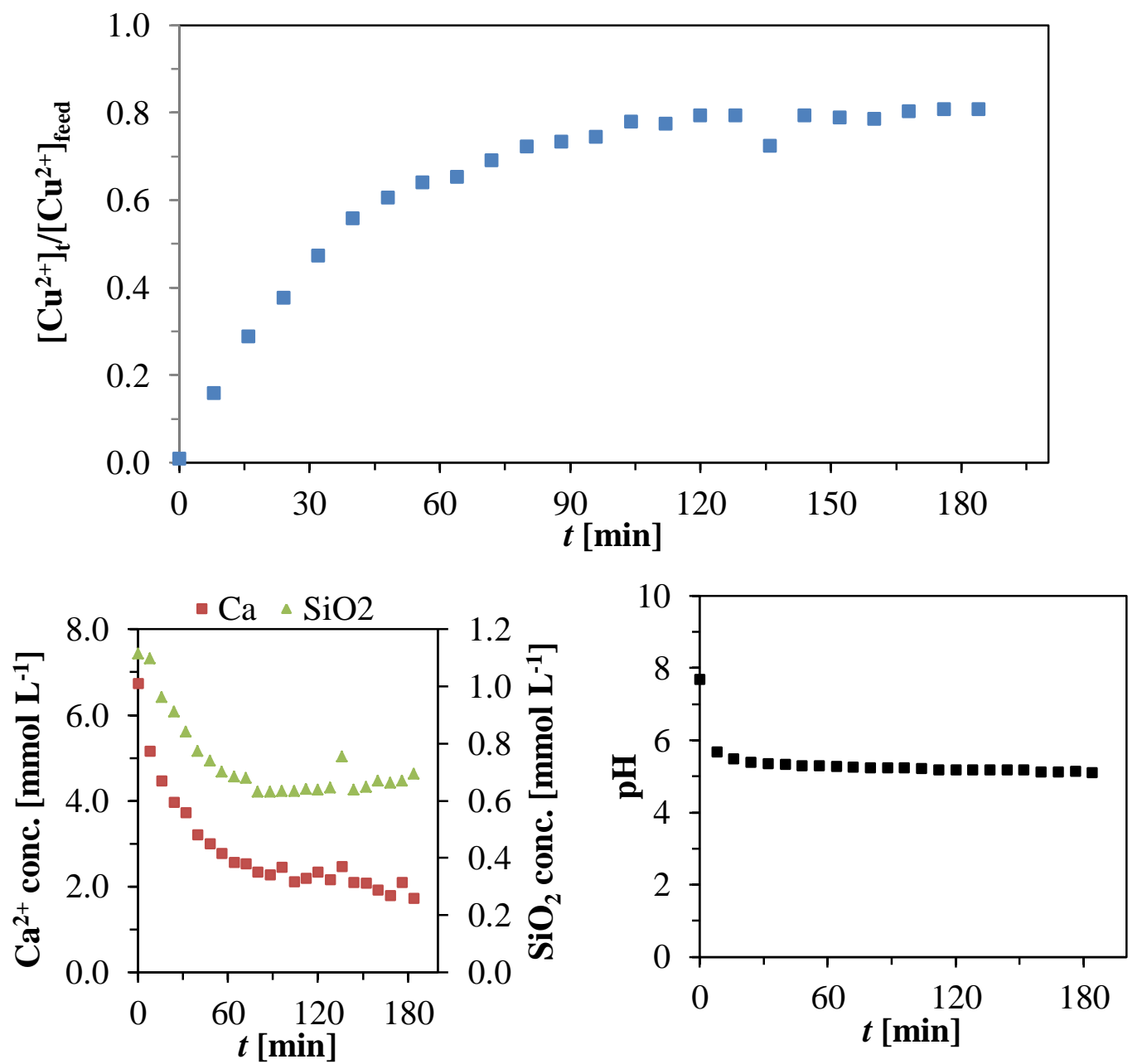

Figure 5.6: Conventional axial flow column experiment for the continuous adsorption of $\mathrm{Cu}^{2+}$ ions onto NCaSil. The graphs present the variation in the concentration of $\mathrm{Cu}^{2+}, \mathrm{Ca}^{2+}$ and monomeric silica as $\mathrm{SiO}_{2}$ in solution over time after it has passed through the column. The change of the pH over time is also shown. Experimental conditions: $\left[\mathrm{Cu}^{2+}\right] 7.9 \mathrm{mmol} \mathrm{L}^{-1}$; bed height $2.5 \mathrm{~cm}$ equivalent to $500 \mathrm{mg}$ of NCaSil packed to bulk density; flow $5 \mathrm{ml} \mathrm{min}^{-1}$; temperature 293 K. Experimental errors are detailed in Table 2.3.

In Figure 5.6 it may be observed that increasing the concentration of $\mathrm{Cu}^{2+}$ approximately five times, from 1.6 to $7.9 \mathrm{mmol} \mathrm{L}^{-1}$, caused the breakthrough time to approach zero. This happened because the MTZ was larger than the bed length for this concentration under the studied conditions.

The release of calcium and monomeric silica from NCaSil followed the same trend and rate as before. Both NCaSil constituents started at high initial concentrations at the outlet of the column with values of 6 and $1 \mathrm{mmol} \mathrm{L}^{-1}$ for calcium and monomeric silica, respectively. The amount of calcium leached was higher than for the experiment done with a $\mathrm{Cu}^{2+}$ concentration of $1.6 \mathrm{mmol} \mathrm{L}^{-1}$.

The $\mathrm{pH}$ value decreased rapidly to a value near 5, close to the value for the feed stock, further indicating a quick breakthrough. Moreover, the pressure drop remained below $15 \mathrm{kPa}$ (not shown) during the experiment. 
Larger concentrations of $15.7 \mathrm{mmol} \mathrm{L}^{-1}$ of $\mathrm{Cu}^{2+}$ were studied since this concentration was the nominal concentration used in kinetics experiments described in Chapter 3. Results of such experiment are shown in Figure 5.7
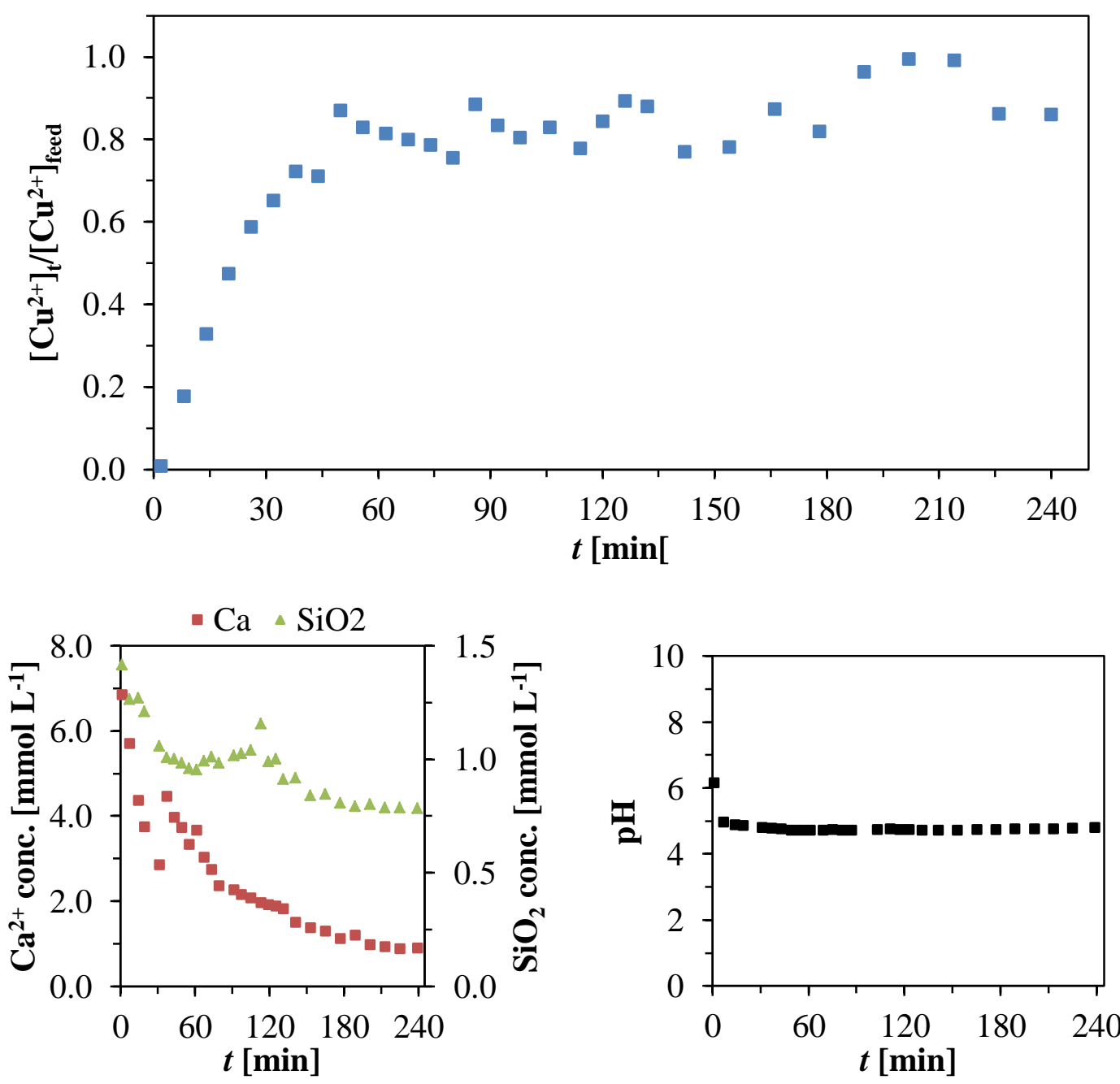

Figure 5.7: Conventional axial flow column experiment for the continuous adsorption of $\mathrm{Cu}^{2+}$ ions onto NCaSil. The graphs present the variation in the concentration of $\mathrm{Cu}^{2+}, \mathrm{Ca}^{2+}$ and monomeric silica as $\mathrm{SiO}_{2}$ in solution over time after it has passed through the column. The change of the pH over time is also shown. Experimental conditions: $\left[\mathrm{Cu}^{2+}\right] 15.7 \mathrm{mmol} \mathrm{L}^{-1}$; bed height $2.5 \mathrm{~cm}$ equivalent to $500 \mathrm{mg}$ of NCaSil packed to bulk density; flow $5 \mathrm{ml} \mathrm{min}^{-1}$; temperature $293 \mathrm{~K}$. Experimental errors are detailed in Table 2.3.

In Figure 5.7 it is clear that utilizing a high concentration of $\mathrm{Cu}^{2+}$ caused an instant breakthrough. The concentration of $\mathrm{Cu}^{2+}$ at the outlet of the column grew at a higher rate than observed in the previous experiment where a $7.9 \mathrm{mmol} \mathrm{L}^{-1} \mathrm{Cu}^{2+}$ solution was used. The column reached exhaustion after approximately $200 \mathrm{~min}$. Afterwards, there is drop in $\mathrm{Cu}^{2+}$ concentration coming out of the column which might be due to copper ions nucleating over wroewolfeite, posnjakite and brochantite crystals which led to the re-accommodation of the packing inside the column which offers exposes fresh NCaSil surface. 
The results shown in Figure 5.5 and Figure 5.6 reveal that a packed column with a bed height to column diameter ratio of 1.25:1 is not suitable for concentrations larger than $1.6 \mathrm{mmol} \mathrm{L}^{-1}$ of $\mathrm{Cu}^{2+}$.

\subsubsection{Experiments using a bed height of $5 \mathrm{~cm}$ of NCaSil}

The bed height was doubled in order to improve the operation time with different concentrations. Nevertheless, doubling the bed length increased the pressure drop inside the column as shown in Eq. 5.6. In the experiments shown throughout this section the pressure drop increased for reasons other than the length. This will be discussed herein. Results for experiments performed with a $5 \mathrm{~cm}$ bed length are shown in Figure 5.8 to Figure 5.10. 

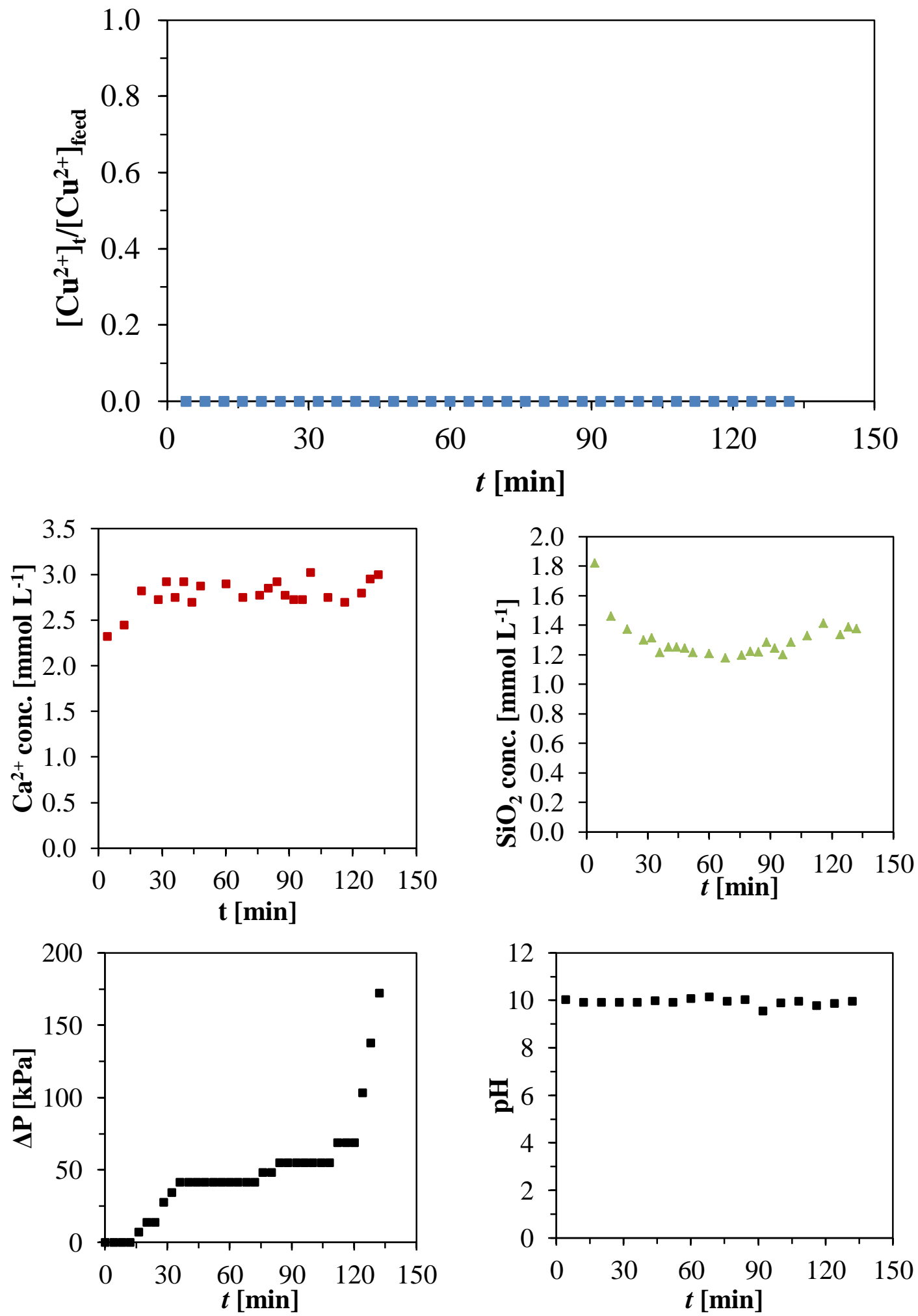

Figure 5.8: Conventional axial flow column experiment for the continuous adsorption of $\mathrm{Cu}^{2+}$ ions onto NCaSil. The graphs present the variation in the concentration of $\mathrm{Cu}^{2+}, \mathrm{Ca}^{2+}$ and monomeric silica as $\mathrm{SiO}_{2}$ in solution over time after it has passed through the column. The change of the pressure and the $\mathrm{pH}$ over time is also shown. Experimental conditions: $\left[\mathrm{Cu}^{2+}\right] 1.6$ mmol $\mathrm{L}^{-1}$; bed height $5 \mathrm{~cm}$ equivalent to $1.00 \mathrm{~g}$ of NCaSil packed to bulk density; flow $5 \mathrm{ml} \mathrm{min}^{-}$ ${ }^{1}$; temperature $293 \mathrm{~K}$. Experimental errors are detailed in Table 2.3.

The experiment carried out using a bed height of $5 \mathrm{~cm}$ and a concentration of $\mathrm{Cu}^{2+}$ of $1.6 \mathrm{mmol} \mathrm{L}^{-1}$ had an unusual behaviour. The experiment had to be stopped due to the large pressure drop inside the column that reached a value near $200 \mathrm{kPa}$, becoming a threat due to the specifications of glassware regarding their pressure tolerance. 
The concentration of $\mathrm{Cu}^{2+}$ at the outlet remained equal to the blank signal for all the studied time.

The release of calcium from NCaSil stayed approximately constant over time, in contrast to the results obtained using a bed height of $2.5 \mathrm{~cm}$ in the previous subsection 5.2.1.1, where a decrease in the concentration over time was observed. Therefore, this is an indication that NCaSil was far from being exhausted when the experiment was stopped.

In the case of the release of monomeric silica from NCaSil, a high concentration was released at the beginning $1.8 \mathrm{mmol} \mathrm{L}^{-1}$ but then dropped and remained constant at a value of $1.2 \mathrm{mmol} \mathrm{L}^{-1}$ for the remaining time for the experiment.

The pressure drop inside in a column should be directly proportional to bed length as shown by the relation in Eq. 5.6. Furthermore, if all variables in Eq. 5.6 during the experiment remain unvaried the pressure drop should be constant throughout the entire experiment. Therefore a change in the pressure would be a clear indication that one or more variables in Eq. 5.6 is changing. In this case the pressure drop was not only exacerbated but increased exponentially during the experiment. This result finds its explanation by the decrease in the void spaces $(\varepsilon)$ inside the column increasing the pressure drop as shown in Eq. 5.6. Most likely due to the formation of amorphous copper hydroxide as discussed in Chapter 3, where no crystalline copper structure could be observed in Figure 3.32 at a concentration of $1.6 \mathrm{mmol} \mathrm{L}^{-1}$. Also the particle diameter could play a role in the increase of the pressure drop which decreases over time when contacted with water as NCaSil disaggregates.

According to filtration theory [118] amorphous solids produce a greater decrease in pressure than crystalline solids due to less void space being present between particles and a tendency to compress. Therefore, raising the concentration should result in the formation of crystalline copper sulfate hydroxide species which will lead to a decrease in the pressure drop. 

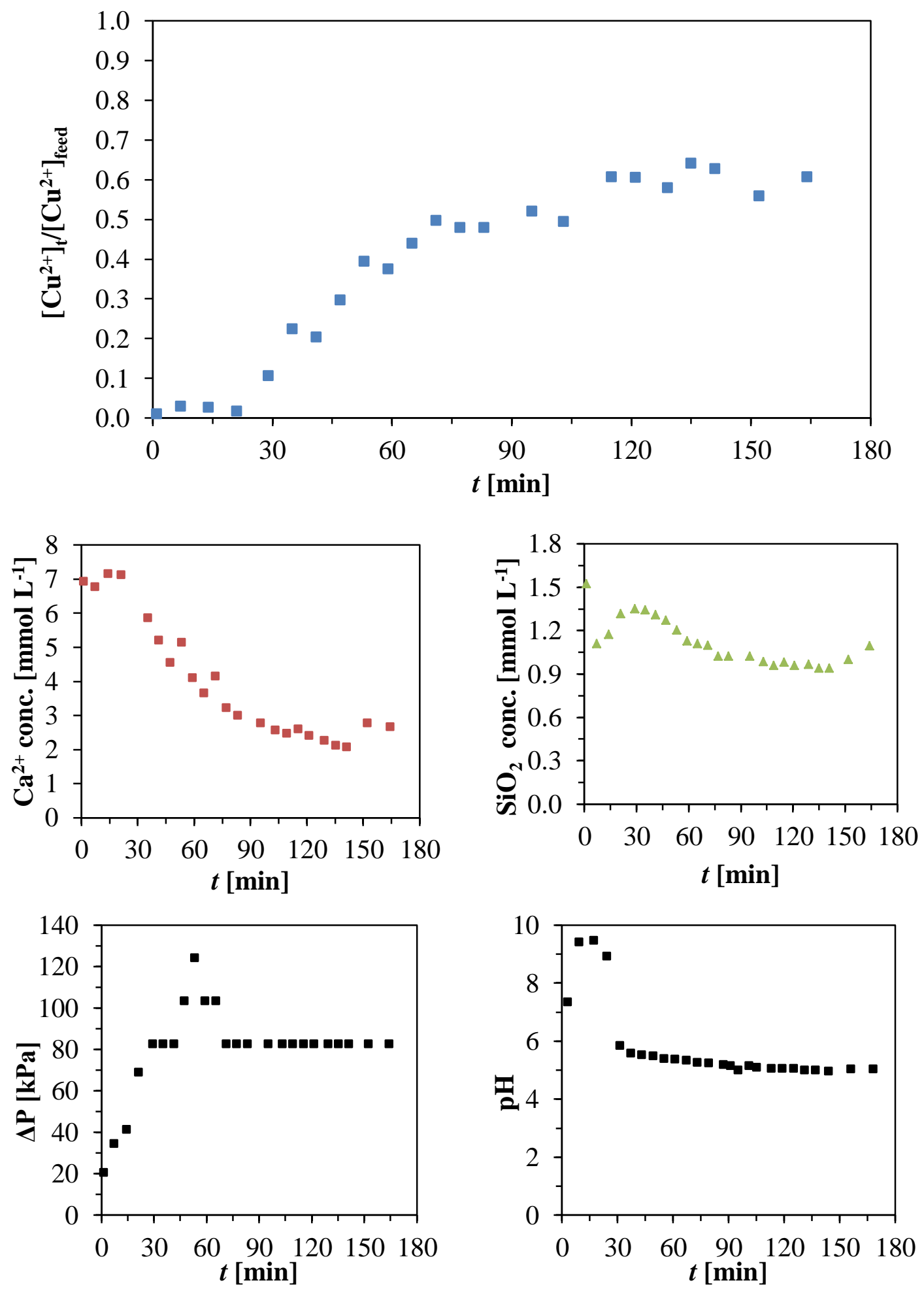

Figure 5.9: Conventional axial flow column experiment for the continuous adsorption of $\mathrm{Cu}^{2+}$ ions onto NCaSil. The graphs present the variation in the concentration of $\mathrm{Cu}^{2+}, \mathrm{Ca}^{2+}$ and monomeric silica as $\mathrm{SiO}_{2}$ in solution over time after it has passed through the column. The change of the pressure and the $\mathrm{pH}$ over time is also shown. Experimental conditions: $\left[\mathrm{Cu}^{2+}\right]$

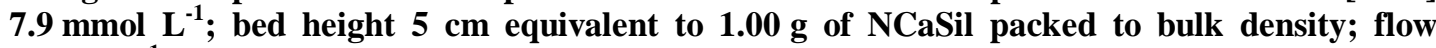
$5 \mathrm{ml} \mathrm{min}^{-1}$; temperature $293 \mathrm{~K}$. Experimental errors are detailed in Table 2.3.

In the experiment summarised in Figure 5.9 the breakthrough occurred 20 minutes after starting. The concentration of $\mathrm{Cu}^{2+}$ increased rapidly at the outlet between 30 and 60 minutes. At 60 minutes the rate at which $\mathrm{Cu}^{2+}$ concentration rose slowed 
down. For the remainder of the experiment the pressure drop grew at a steady rate. The column didn't reach exhaustion.

The release of calcium from $\mathrm{NCaSil}$ into solution remained unvaried near a value of $7 \mathrm{mmol} \mathrm{L} \mathrm{L}^{-1}$ during the first 20 minutes, which was coincidental with the time frame where the outlet $\mathrm{Cu}^{2+}$ concentration did not change, suggesting a direct relation. Subsequently, the release of calcium was proportional to the uptake of copper.

The release of monomeric silica lessened in the first ten minutes and then increased until it reached a momentary maximum at 30 minutes. This coincided with the time of breakthrough of $\mathrm{Cu}^{2+}$ into solution. Then the concentration of silica at the outlet dropped to a value of $1 \mathrm{mmol} \mathrm{L}^{-1}$ and remained unvaried until the end of the experiment.

The pressure drop grew at a steady rate until it reached $120 \mathrm{kPa}$ and then dropped at a similar rate to a value of $80 \mathrm{kPa}$ remaining unvaried for the rest of the experiment.

The $\mathrm{pH}$ value increased to a value near 10 during the first $30 \mathrm{~min}$ of experiment. Then it suddenly dropped reaching a value near 5.8.

The pressure variation throughout the experiment may be directly related to the changes in the packing material. At the beginning the pressure rose most likely due to amorphous hydroxide being formed blocking the interstitial volume. After reaching a maximum value of $130 \mathrm{kPa}$ the pressure dropped. This is attributable to the formation of copper sulfate hydroxide minerals which are not blocking pores and void spaces to the same degree as the initially formed amorphous copper hydroxide. After this crystallization the pressure remained unvaried throughout the experiment suggesting that $\varepsilon$ and $D_{p}$ remained unvaried as well.

Increasing the concentration of copper ions in solution further should lessen the pressure drop, mainly due to the faster formation of copper hydroxide sulfate crystals (see Chapter 3). This takes into consideration the pressure drop trends shown in Figure 5.8 and Figure 5.9. Results for a $\mathrm{Cu}^{2+}$ concentration of $15.7 \mathrm{mmol} \mathrm{L}^{-1}$ are shown in Figure 5.10. 

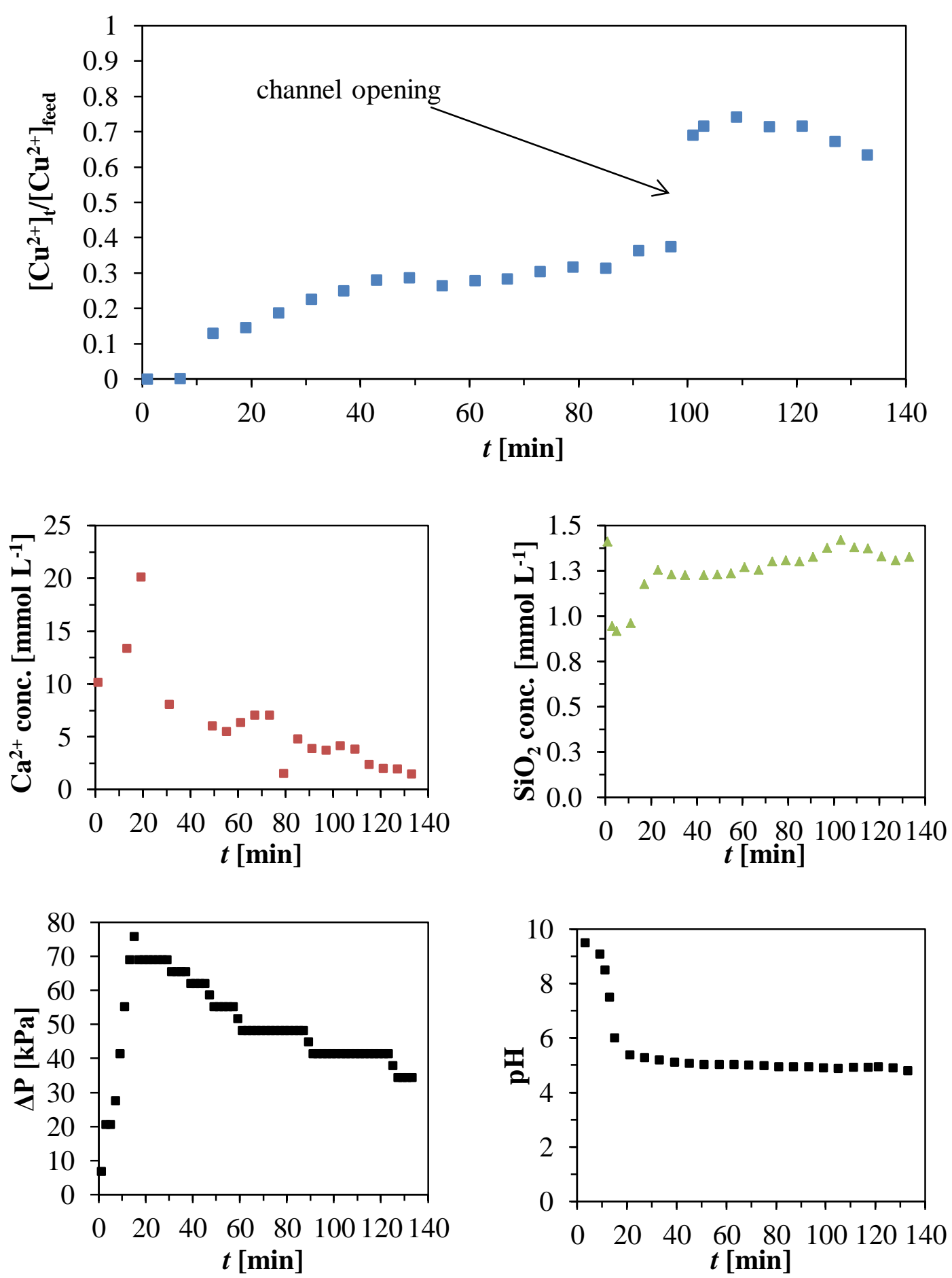

Figure 5.10: Conventional axial flow column experiment for the continuous adsorption of $\mathrm{Cu}^{2+}$ ions onto NCaSil. The graphs present the variation in the concentration of $\mathrm{Cu}^{2+}, \mathrm{Ca}^{2+}$ and monomeric silica as $\mathrm{SiO}_{2}$ in solution over time after it has passed through the column. The change of the pressure and the $\mathrm{pH}$ over time is also shown. Experimental conditions: $\left[\mathrm{Cu}^{2+}\right] 15.7$ mmol L ${ }^{-1}$; bed height $5 \mathrm{~cm}$ equivalent to $1.00 \mathrm{~g}$ of NCaSil packed to bulk density; flow $5 \mathrm{ml} \mathrm{min}$ ${ }^{1}$; temperature $293 \mathrm{~K}$. Experimental errors are detailed in Table 2.3.

In Figure 5.10 the breakthrough of copper concentration at the outlet for an experiment using $15.7 \mathrm{mmol} \mathrm{L}^{-1}$ of $\mathrm{Cu}^{2+}$ solution was reached after approximately $10 \mathrm{~min}$ from the start. This was roughly half the time needed to reach the breakthrough when compared to the experiment summarised in Figure 5.9. Therefore, it could be possible to say that the breakthrough time is linearly dependant 
on the concentration as long as all other variables remain constant. Furthermore, there was a segment from about 45 to 100 minutes where the outlet concentration remained roughly constant around $0.3 \mathrm{mmol} \mathrm{L}^{-1}$ followed by another breakthrough after $100 \mathrm{~min}$ of starting the experiment. The levelling-out, still at elevated copper outlet concentration, was most likely due to the formation of free channels inside the column which offered the solution the possibility to pass quickly avoiding interaction with the packing. This behaviour was also considered to be due to changes in the packing morphology and spatial distribution as indicated by leaching of calcium and monomeric silica, which inevitably impacted on the changes the particles morphology. The variation of the pressure drop in the column during the experiment supports further this statement which is direct relation to particles morphology and void spaces inside the column as shown in Eq. 5.6.

The rate of calcium release increased rapidly in the first twenty minutes reaching a maximum value of $20 \mathrm{mmol} \mathrm{L}^{-1}$. Then it decreased rapidly to a value near $8 \mathrm{mmol} \mathrm{L}^{-}$ ${ }^{1}$ at $30 \mathrm{~min}$ and for the rest of the experiment showed a linear steady decrease until the end of experiment. The calcium concentration was very low at the end of experiment and considering the steady rate of decrease of concentration, it is possible to extrapolate that after $160 \mathrm{~min}$ the concentration of calcium present at the outlet of the column should be below $0.01 \mathrm{mmol} \mathrm{\textrm {L } ^ { - 1 }}$.

The release of monomeric silica decreased dramatically during the first $10 \mathrm{~min}$ of the reaction. Between 10 and 20 minutes it rose rapidly to a value of $1.3 \mathrm{mmol} \mathrm{L}^{-1}$, where it stayed unvaried for the rest of the experiment.

The pressure drop increased rapidly during the first 20 minutes reaching a value of $80 \mathrm{kPa}$. Then it dropped for the rest of the experiment reaching a value of 40 at the end of the experiment. Comparing the results regarding pressure drop shown in Figure 5.8 and Figure 5.9 with those shown in Figure 5.10, it may be observed that the pressure drop inside the column was lower with a higher $\mathrm{Cu}^{2+}$ concentration in the feed. This supports the statement regarding the formation of crystalline copper sulfate hydroxide species made earlier, where their formation if promoted by increasing concentration as shown in Chapter 3. In fact, the final pressure drop value in Figure 5.10 is half of that shown in Figure 5.9. The average pressure drop under the experimental conditions in Figure 5.10 was approximately $10 \mathrm{kPa}$ per $\mathrm{cm}(10 \mathrm{~atm}$ per metre) of bed height. This value is too high for a practical application of NCaSil in such setup. 
During the first 20 minutes the value of the $\mathrm{pH}$ decreased rapidly from a value near 9.5 to a value of 6 . During the rest of the experiment the value of the $\mathrm{pH}$ approached 5 very slowly. Copper sulfate hydroxide crystals form readily at this $\mathrm{pH}$ value as shown in Chapter 3.

Under the studied conditions it was possible to operate the column without large pressure drops. Nonetheless, a total of 20 minutes of operational time is not good enough to suggest scale-up in an axial column set. In addition, the hydraulic loading was $0.05 \mathrm{~m}^{3} \mathrm{~m}^{-2} \mathrm{~min}^{-1}$, hence was one order of magnitude less than what is usually used in the industry. Also, the pressure drop was an average of $16 \mathrm{kPa} \mathrm{cm}^{-1}$ which is 10 to 100 larger than what is expected for a packed column as mentioned in subsection 5.1.1. Thus a ratio of bed height to column internal diameter of 2.5:1 is not practical. 


\subsubsection{Continuous uptake of $\mathrm{Cu}^{2+}$ onto NCaSil using a radial flow}

Results shown in subsection 5.2.1 were proof that using NCaSil inside a standard axial flow column produced large pressure drops. Therefore a radial flow column (RFC) was chosen in order to increase the cross sectional area which in turn decreases the pressure drop inside the column by decreasing the hydraulic loading (at constant flow). In addition, the footprint (the amount of space a potential device uses) is not enlarged dramatically.

Results obtained using the RFC in a continuous manner are shown from Figure 5.11 to Figure 5.13.
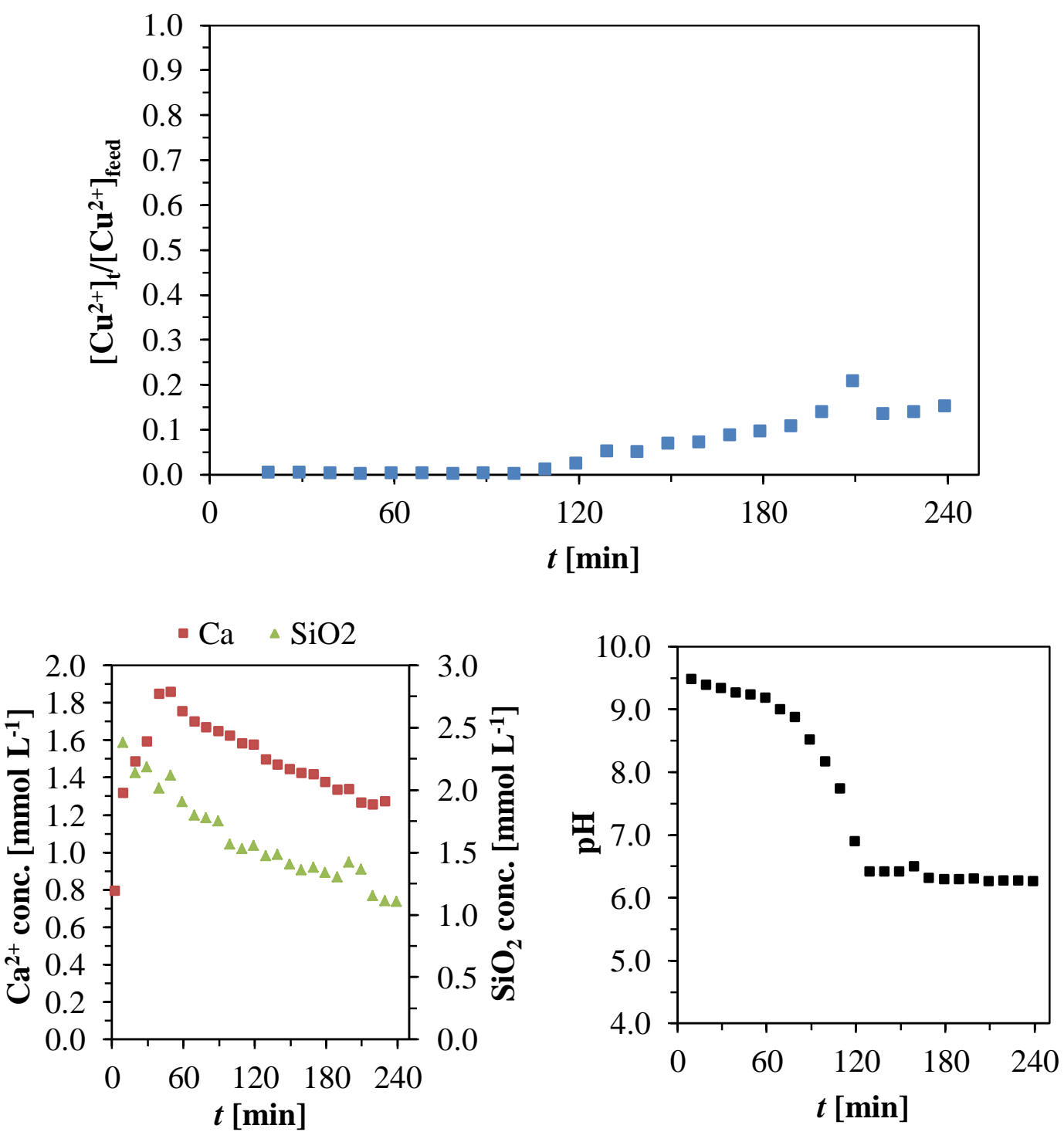

Figure 5.11: RFC experiment for the continuous adsorption of $\mathrm{Cu}^{2+}$ ions onto NCaSil. The graphs present the variation in the concentration of $\mathrm{Cu}^{2+}, \mathrm{Ca}^{2+}$ and monomeric silica $\mathrm{as} \mathrm{SiO}_{2}$ in solution over time after it has passed through the column. Experimental conditions: $\left[\mathrm{Cu}^{2+}\right]_{\text {feed }}$ $1.6 \mathrm{mmol} \mathrm{L}^{-1}$; logarithmic mean cross sectional area of $7.3 \times 10^{-3} \mathrm{~m}^{2}$; bed height $2 \mathrm{~cm} ; 3.0 \mathrm{~g} \mathrm{of}$ NCaSil packed to bulk density; flow $10 \mathrm{ml} \mathrm{min}^{-1}$; temperature $293 \mathrm{~K}$. Experimental errors are detailed in Table 2.3. 
In the experiment summarised in Figure 5.11 the concentration of $\mathrm{Cu}^{2+}$ remained low throughout the first $100 \mathrm{~min}$ of the experiment. The breakthrough occurred after 120 min with the outlet concentration of copper increasing steadily for the rest of the experiment. The column did not reach exhaustion. A sudden peak was observed at 210 minutes after starting the experiment which could be attributed to a slight packing rearrangement as copper is adsorbed and monomeric silica and calcium leach out from NCaSil.

The release of calcium into solution rose during the first hour of experiment and reached a maximum value of $1.9 \mathrm{mmol} \mathrm{L}^{-1}$. Then it declined at a steady rate for the rest of the experiment reaching to a value of $1.4 \mathrm{mmol} \mathrm{L}^{-1}$. Hence exhaustion of calcium did not occur either.

The concentration of monomeric silica fell at a steady rate, from a value 2.2 to a value of $1.1 \mathrm{mmol} \mathrm{L}^{-1}$, at the end of the experiment.

The $\mathrm{pH}$ value of the outlet stream decreased linearly during the first hour from a value of 9.5 to a value of 9.2. Then the $\mathrm{pH}$ fell rapidly, with increasing rate, reaching a value of 6.5 after 2 hours after which it levelled out for the rest of the experiments.

The variation in the pressure drop throughout the experiment was below $10 \mathrm{kPa}$ (not shown).

The slow increase in the concentration of $\mathrm{Cu}^{2+}$ was considered to be either a result of copper nucleating over crystals or due to intraparticle diffusion.

The value of the $\mathrm{pH}$ followed an inverse relation to the amount of $\mathrm{Cu}^{2+}$ present at the outlet of the column. 

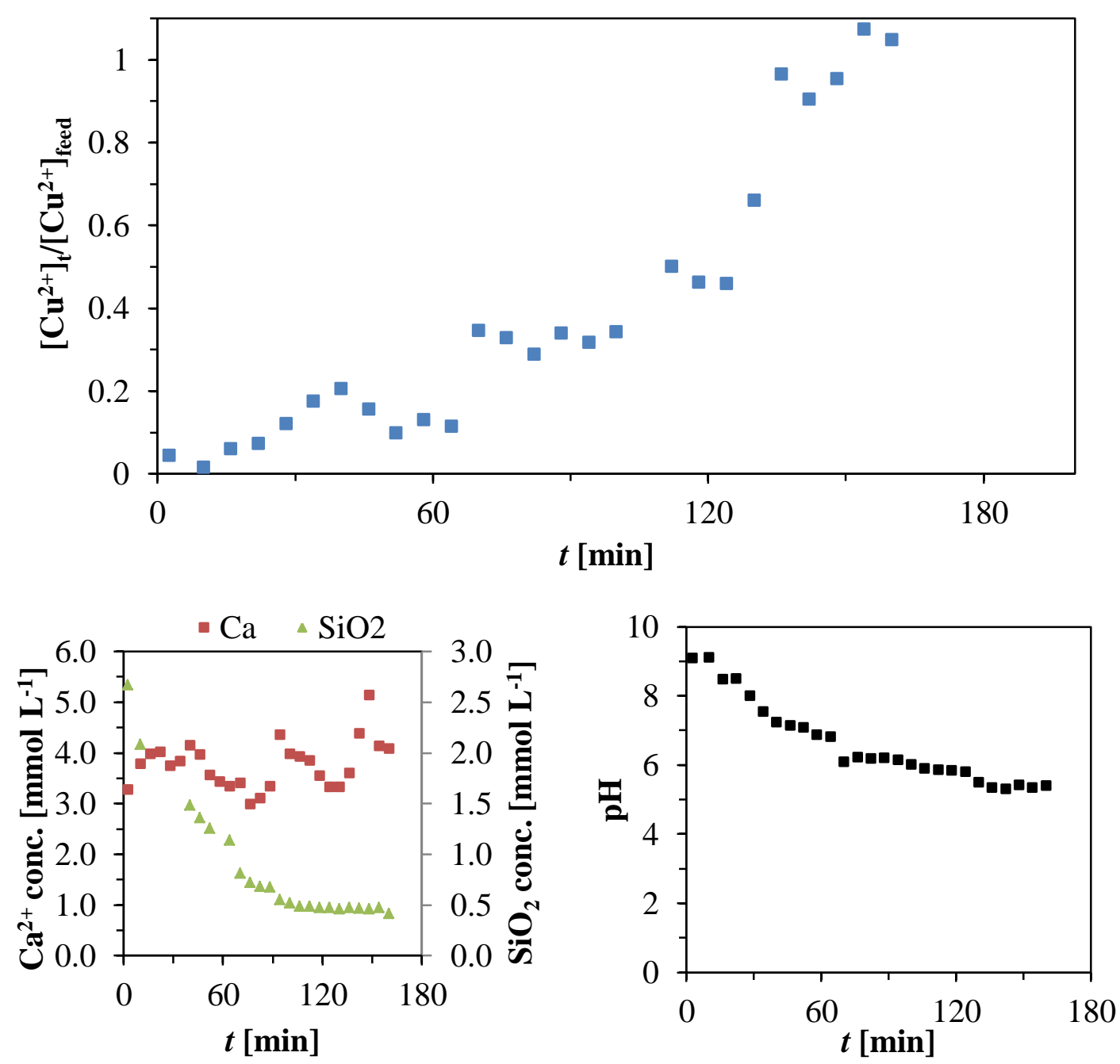

Figure 5.12: RFC experiment for the continuous adsorption of $\mathrm{Cu}^{2+}$ ions onto NCaSil. The graphs present the variation in the concentration of $\mathrm{Cu}^{2+}, \mathrm{Ca}^{2+}$ and monomeric silica as $\mathrm{SiO}_{2}$ in solution over time after it has passed through the column. Experimental conditions: $\left[\mathrm{Cu}^{2+}\right]_{\text {feed }}$ $7.9 \mathrm{mmol} \mathrm{L}^{-1}$; logarithmic mean cross sectional area of $7.3 \times 10^{-3} \mathrm{~m}^{2}$; bed height $2 \mathrm{~cm} ; 3.0 \mathrm{~g}$ of NCaSil packed to bulk density; flow $10 \mathrm{ml} \mathrm{min}^{-1}$; temperature $293 \mathrm{~K}$. Experimental errors are detailed in Table 2.3.

In Figure 5.12 a breakthrough occurring after 10 min can be observed, earlier than the results shown in Figure 5.11. The concentration of $\mathrm{Cu}^{2+}$ at the beginning in the first data point was near $5 \%$ of the inlet concentration and then lowered to near $1 \%$. The concentration of $\mathrm{Cu}^{2+}$ rose to an early maximum of $20 \%$ near the 40 minute mark, but then fell to a value near $10 \%$. After 40 minutes the outlet concentration of $\mathrm{Cu}^{2+}$ increased reaching exhaustion after 150 minutes of starting the experiment.

The release of calcium varied with a certain periodicity throughout the studied time frame, with an average value of $3.8 \mathrm{mmol} \mathrm{L}^{-1}$. 
The release of monomeric silica into solution decreased in an exponential manner from an initial value of $2.7 \mathrm{mmol} \mathrm{L}^{-1}$ to a final value of $0.4 \mathrm{mmol} \mathrm{L}^{-1}$ at the end of the experiment.

The value of the $\mathrm{pH}$ had a decreased at a steady rate from a value of 9.5 to a value near 6 at the end of the experiment.

The early maximum in the breakthrough curve was most probably due to the formation of free channels within packing. The drop in copper concentration after the early maximum was probably due to the rearrangement of $\mathrm{NCaSil}$ particles inside the column and/or the blockage of the free channels by the formation of copper hydroxide on the surface of NCaSil. Also, re-arrangement of the packing inside the column could have been promoted by leaching of calcium and monomeric silica. 

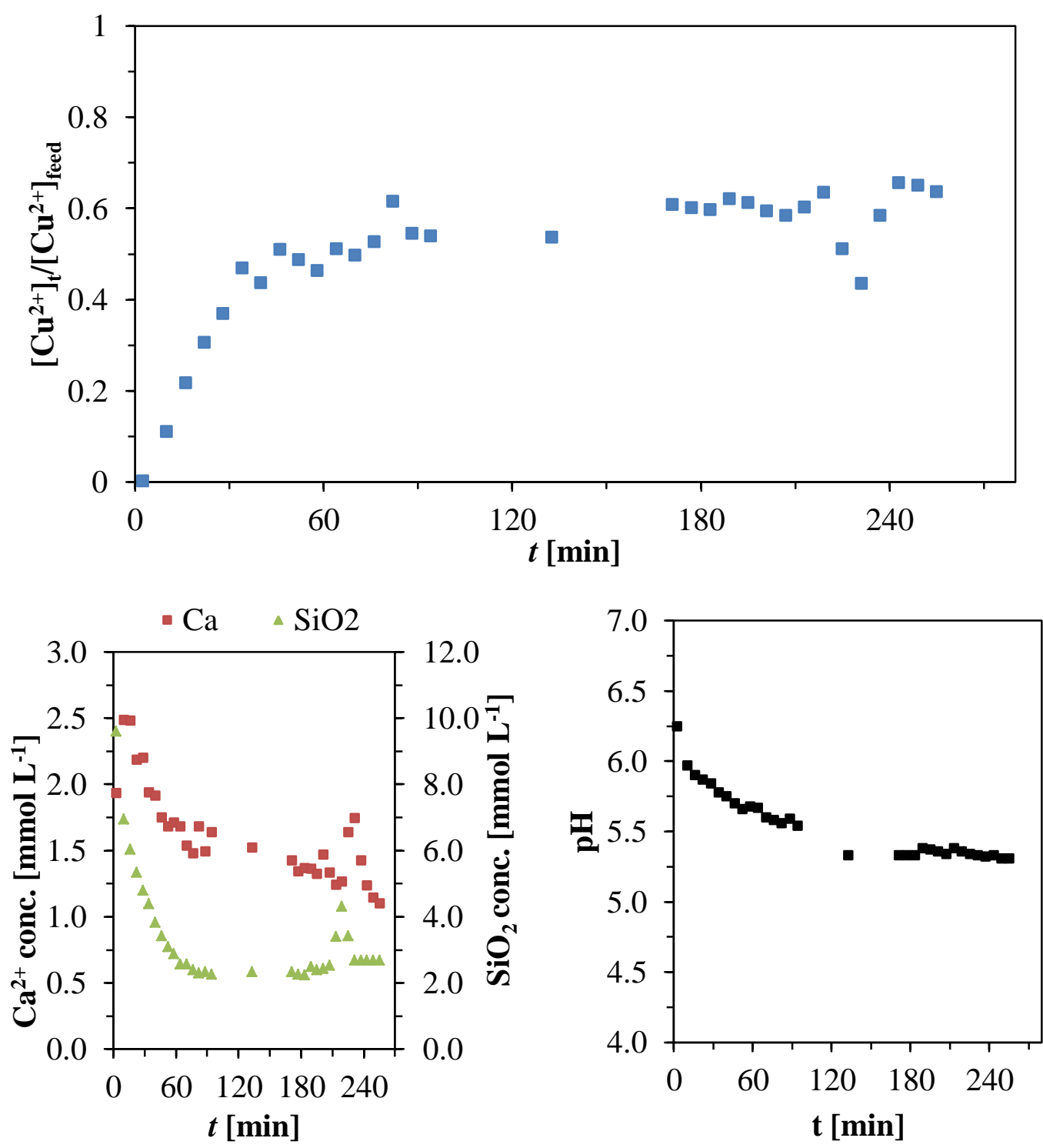

Figure 5.13: RFC experiment for the continuous adsorption of $\mathrm{Cu}^{2+}$ ions onto NCaSil. The graphs present the variation in the concentration of $\mathrm{Cu}^{2+}, \mathrm{Ca}^{2+}$ and monomeric silica as $\mathrm{SiO}_{2}$ in solution over time after it has passed through the column. Experimental conditions: $\left[\mathrm{Cu}^{2+}\right]_{\text {feed }}$ $15.7 \mathrm{mmol} \mathrm{L}^{-1}$; logarithmic mean cross sectional area of $7.3 \times 10^{-3} \mathrm{~m}^{2}$; bed height $2 \mathrm{~cm} ; 3.0 \mathrm{~g}$ of NCaSil packed to bulk density; flow $10 \mathrm{ml} \mathrm{min}^{-1}$; temperature $293 \mathrm{~K}$. Experimental errors are detailed in Table 2.3.

In the experiment summarised in Figure 5.13 the breakthrough occurred almost instantaneously, indicating that the MTZ was larger than the bed length for these particular experimental conditions. The concentration of $\mathrm{Cu}^{2+}$ rose rapidly during the first 30 minutes. After this it slowed down decreasing at a steady rate for the rest of the experiment. There was a sudden drop in the concentration of copper near $240 \mathrm{~min}$ after starting the experiment. The column didn't reach exhaustion.

The release of calcium and monomeric silica followed the same trend. Both analytes were released in large quantities at the beginning of the experiment. The concentration of both analytes fell rapidly over time until 60 minutes after starting 
the experiment. Subsequently the leaching of monomeric silica levelled out for the remainder of the experiment, while the concentration of calcium being leached decreased linearly with time. Nonetheless, both analytes showed a peak in their concentrations coinciding with the time at which a peak in the copper release was observed.

The $\mathrm{pH}$ value decreased over time from a value 6.4 to a value of 5.3 during the experiment.

The sudden drop in the concentration of $\mathrm{Cu}^{2+}$ was unlikely due to exposure to a fresh surface of NCaSil, since the concentration of calcium and monomeric silica also rose during the same period. This indicated that NCaSil may disaggregate inside the column. This is troublesome as this changes the column packing. These peaks were not related to problems in the way the column was packed initially. If that was the case, peaks for copper, calcium and monomeric silica concentration would have appeared considerably earlier in the experiment.

Therefore, NCaSil displays an unreliable behaviour in a continuous column-type system, probably due to the leaching of its constituents which lead to a rearrangement of the packing, presenting sudden peaks or drops in the concentration of $\mathrm{Cu}^{2+}$ at the outlet.

Nonetheless, the RFC proved to be a significant improvement over a standard column being able to operate without pressure drops with twice the flow and with a similar footprint. 


\subsubsection{Continuous uptake of $\mathrm{Cu}^{2+}$ onto $\mathrm{NCaSil}$ using a radial flow column} immersed in a tank containing a copper sulfate solution.

$\mathrm{NCaSil}$ releases calcium ions and monomeric silica into the solution as seen in Chapter 3. Although other materials commonly used in water treatment like ion exchange resins and aluminosilicate materials also do so, NCaSil additionally undergoes structural changes during operation. As it was shown in section 5.2.1, these changes led to large pressure drops which varied throughout the experiment/operation. Using a radial flow column as described in section 5.2.2 solved some of the problems associated with the large pressure drops. Nevertheless, due to a reduction in the bed length, operational times were too short to offer promising scale-up projections.

In order to find a procedure that combines the strengths of NCaSil and the RFC setup a semi-batch approach was chosen. The RFC was operated by immersion in a stirred tank where the contaminated solution inside the tank was recirculated through the device (refer to Figure 2.6 in section 2.5.3.3). Experiments were performed keeping the same adsorbent dosage (1 gram of adsorbent per 1 litre of solution to be treated) used in Chapter 3. Data collected from these experiments are shown in Figure 5.14 and Figure 5.15. 

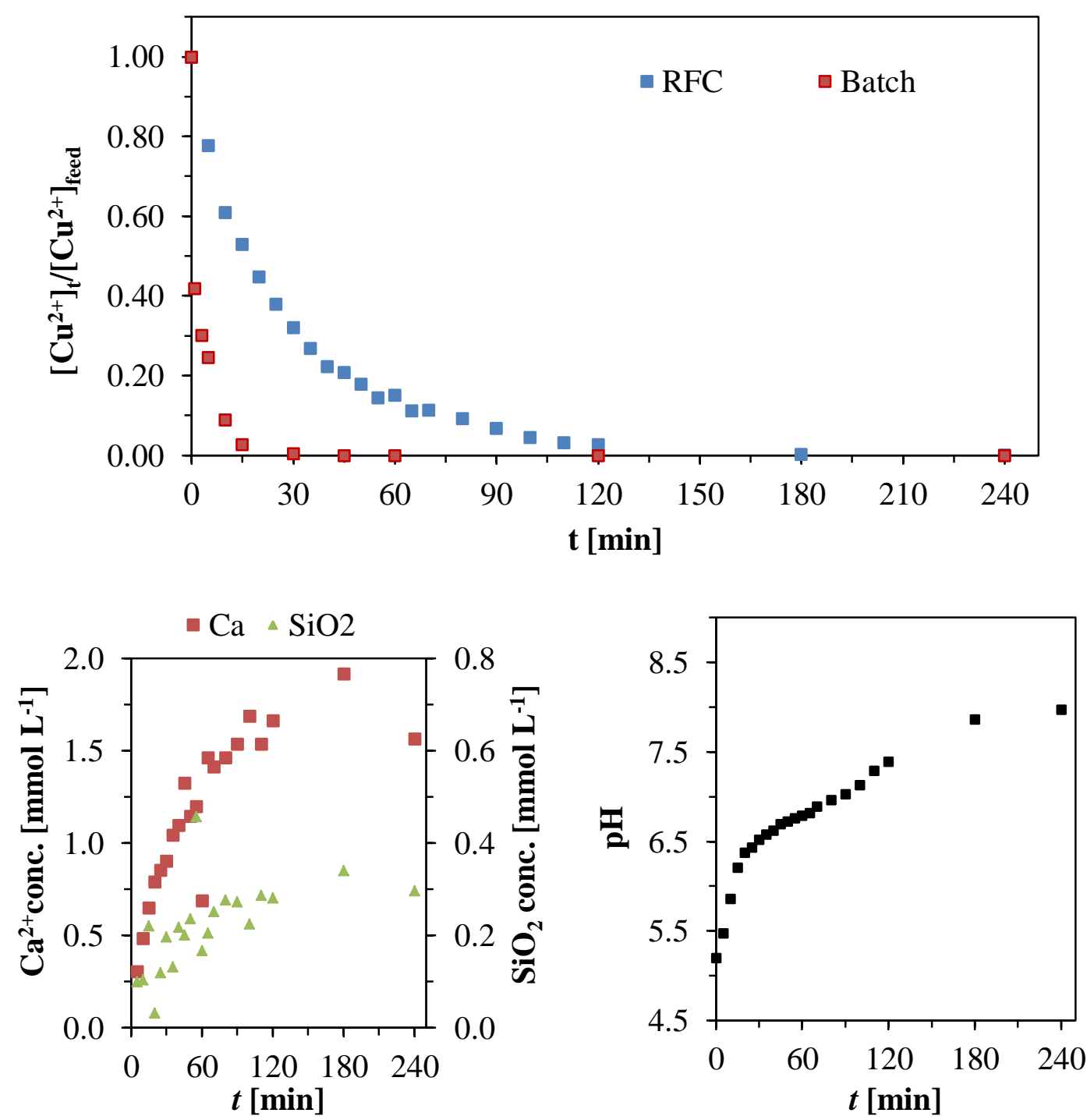

Figure 5.14: RFC experiment for the continuous adsorption of $\mathrm{Cu}^{2+}$ ions onto NCaSil. The graphs present the variation in the concentration of $\mathrm{Cu}^{2+}, \mathrm{Ca}^{2+}$ and monomeric silica as $\mathrm{SiO}_{2}$ in solution over time after it has passed through the column. The variation of the concentration of $\mathrm{Cu}^{2+}$ over time for the same initial concentration of $\mathrm{Cu}^{2+}$ is also presented. Experimental conditions batch: $\left[\mathrm{Cu}^{2+}\right]_{\text {initial }} 7.9 \mathrm{mmol} \mathrm{L}^{-1}$; adsorbent dosage $1 \mathrm{~g} \mathrm{~L}^{-1}$; temperature $293 \mathrm{~K}$; stirring speed $500 \mathrm{rpm}$. Experimental conditions RFC: $\left[\mathrm{Cu}^{2+}\right]_{\text {feed }} 1.6 \mathrm{mmol} \mathrm{L}^{-1}$; logarithmic mean cross sectional area of $7.3 \times 10^{-3} \mathrm{~m}^{2}$; bed height $2 \mathrm{~cm} ; 3.0 \mathrm{~g}$ of NCaSil packed to bulk density; flow $200 \mathrm{ml} \mathrm{min}^{-1}$; temperature $293 \mathrm{~K}$. Experimental errors are detailed in Table 2.3.

In Figure 5.14 almost all $\mathrm{Cu}^{2+}$ is absorbed after $180 \mathrm{~min}$. It is noteworthy that the concentration of $\mathrm{Cu}^{2+}$ decreased in a similar manner to that observed in a batch reactor. Nonetheless, the time to reach equilibrium was 6 times longer.

The release of calcium into solution rose until it reached a value of $1.9 \mathrm{mmol} \mathrm{L}^{-1}$ after $180 \mathrm{~min}$ and then decreased to a value of $1.6 \mathrm{mmol} \mathrm{L}^{-1}$, with a similar release rate behaviour to that exhibited in batch reactors in Chapter 3.

The release of monomeric silica into solution followed the same trend as calcium over time reaching a value near $0.3 \mathrm{mmol} \mathrm{L}{ }^{-1}$. 
The variation in the value of the $\mathrm{pH}$ over time resembled the one observed in Figure 3.8 in section 3.25 for batch experiments. The $\mathrm{pH}$ rose rapidly during the first 25 minutes of the reaction from a value equal to 5.3 to a value of 6.5 . Then it slowed down and grew linearly with time until it reached a value near 8.0 after $180 \mathrm{~min}$. Thereafter, the $\mathrm{pH}$ value remained unvaried.

The results are very promising since at a relatively high initial concentration of $\mathrm{Cu}^{2+}$ (1.6 mmol L ${ }^{-1}$ ) all of copper was removed readily form solution within $180 \mathrm{~min}$.

In the case of real liquid waste containing heavy metals in solution, the final $\mathrm{pH}$ of the solution near 8.0 allows the possibility of precipitating other metal hydroxides. It is most likely but not proven that NCaSil could act not only as an adsorbent but also as a filter media retaining metal hydroxide precipitates within NCaSil particles when the solution is recirculated. The trend of the $\mathrm{pH}$ variation over time suggested a close relation between this experimental setup and batch reactors.

The pressure drop throughout the experiment remained below $10 \mathrm{kPa}$, thus, suggesting the possibility for a scaled-up setup under this studied conditions.

The column was immersed in a solution containing $7.9 \mathrm{mmol} \mathrm{L}^{-1}$ of $\mathrm{Cu}^{2+}$ in order to observe how this variable will affect the uptake kinetics using the immersion cartridge. Results of such experiment are shown in Figure 5.15. 

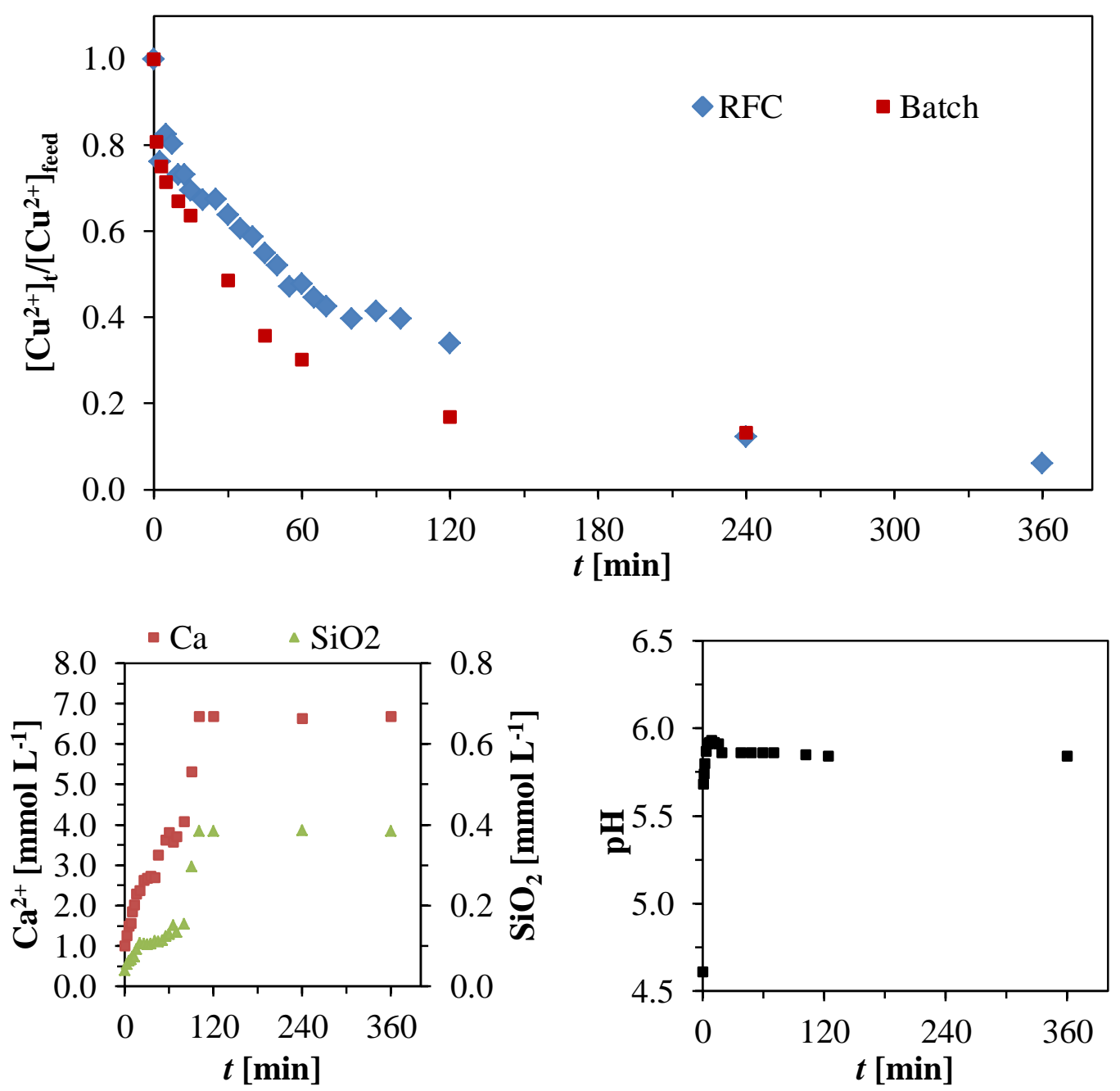

Figure 5.15: RFC experiment for the continuous adsorption of $\mathrm{Cu}^{2+}$ ions onto NCaSil. The graphs present the variation in the concentration of $\mathrm{Cu}^{2+}, \mathrm{Ca}^{2+}$ and monomeric silica as $\mathrm{SiO}_{2}$ in solution over time after it has passed through the column. The variation of the concentration of $\mathrm{Cu}^{2+}$ over time for the same initial concentration of $\mathrm{Cu}^{2+}$ is also presented. Experimental conditions batch: $\left[\mathrm{Cu}^{2+}\right]_{\text {initial }} 7.9 \mathrm{mmol} \mathrm{L}^{-1}$; adsorbent dosage $1 \mathrm{~g} \mathrm{~L}^{-1}$; temperature $293 \mathrm{~K}$; stirring speed $500 \mathrm{rpm}$. Experimental conditions RFC: $\left[\mathrm{Cu}^{2+}\right]_{\text {feed }} 7.9 \mathrm{mmol} \mathrm{\textrm {L } ^ { - 1 }}$; logarithmic mean cross sectional area of $7.3 \times 10^{-3} \mathrm{~m}^{2}$; bed width $2 \mathrm{~cm} ; 3.0 \mathrm{~g}$ of NCaSil packed to bulk density; flow $200 \mathrm{ml} \mathrm{min}^{-1}$; temperature $293 \mathrm{~K}$. Experimental errors are detailed in Table 2.3.

In Figure 5.15 it can be seen that almost all $\mathrm{Cu}^{2+}$ was absorbed after $360 \mathrm{~min}$. The concentration of $\mathrm{Cu}^{2+}$ fell in a similar manner to what would be observed in a batch reactor. The concentration of $\mathrm{Cu}^{2+}$ decreased faster in a batch setup for the first 240 minutes. After this time it takes a further 1200 minutes to reach a similar endpoint value than the one obtained for the RFC at 360 minutes.

The release of calcium and monomeric silica into solution followed a sigmoidal behaviour before levelling off at a maximum after 120 minutes.

The $\mathrm{pH}$ variation over time is similar to the one observed in Figure 3.8 in section 3.2.5 for the release of $\mathrm{OH}^{-}$in an experiment done in batch at the same concentration and temperature. 
These results are very promising since at a relatively high initial concentration $\left(7.9 \mathrm{mmol} \mathrm{L}^{-1}\right) \mathrm{Cu}^{2+}$ was almost completely removed from solution after $360 \mathrm{~min}$.

Moreover, the pressure drop throughout the experiment remained below $10 \mathrm{kPa}$, thus again meeting the criteria for a potential scale-up under the studied conditions.

The immersion procedure looks promising as it offers a similar behaviour to that exhibited in batch but without the need of a separate filtration stage. The advantages and disadvantages are summarised in Table 5.1.

Table 5.1: Advantages and disadvantages of operating the radial flow column in a semi-batch manner by immersion inside a tank.

\begin{tabular}{|c|c|}
\hline Advantages & Disadvantages \\
\hline $\begin{array}{l}\text { - The operation is similar to a batch } \\
\text { reactor but without the need of an } \\
\text { extra filtration step. } \\
\text { - NCaSil is used almost to its full } \\
\text { capacity, in contrast to column } \\
\text { operations where, after the } \\
\text { breakthrough NCaSil still has } \\
\text { available capacity. } \\
\text { - No high pressure drops compared } \\
\text { to columns. } \\
\text { - No extensive care has to be taken } \\
\text { when the material is packed. } \\
\text { May filter other suspended solids } \\
\text { in solution by retaining them } \\
\text { within the NCaSil particles. }\end{array}$ & $\begin{array}{l}\text { - Longer operational times needed } \\
\text { than in batch reactors. } \\
\text { - Non-continuous production of } \\
\text { treated water compared to column } \\
\text { operation. }\end{array}$ \\
\hline
\end{tabular}

1.- Nowadays some of the research focuses on adsorbent magnetic particles [34, 37] that can be removed from solution without the need of filtration. Therefore this approach to some degree competes with the use of these highly engineered magnetic materials.

From Table 5.1 it becomes clear that operating the RFC by immersion offers an advantage over standard batch processes and column chromatography under most conditions due to cost savings by avoiding extra filtration and using the adsorbent close to its capacity. Although it cannot fully compete with columns regarding the continuous production of treated water in some cases it might be competitive if tanks 
or pools of $1-100 \mathrm{~m}^{3}$ containing a waste solution with heavy metals have to be cleaned.

Moreover, in the next sub section the scale-up of this process will be addressed in order to obtain the correct sizing of the equipment considering the results obtained in Chapter 3 and 5.

\subsubsection{Scale-up of the radial flow column used as an immersion device.}

In sub section 5.2.3 it was proven that the RFC works very well for the removal of $\mathrm{Cu}^{2+}$ from solution by immersion of the device in a tank and recirculating the solution through the it.

The scale-up of this process should consider the following recommendations:

- The ratio of inner feed cylinder diameter to external diameter to length of the of an RFC should be kept constant. Example: inner feed cylinder diameter $\left(D_{\text {int }}\right) 2 \mathrm{~cm}$; sorbent holder diameter $\left(D_{S B}\right): 4$; cartridge length $\left(C_{L}\right) 8 \mathrm{~cm}$; Therefore the ratio of Dint to $D_{S B}$ to $C_{L}$ is $a: 2 a: 4 a$, where $a$ would be $D_{\text {int }}$ length in m. An example is shown in Figure 5.16.

- Use ethanol-washed NCaSil if possible that has been passed through a 10 to 20 mesh. This will create granules/aggregates of NCaSil easy to handle in order to fill the RFC.

- Use a hydraulic loading of $1.8 \mathrm{~m}^{3} \mathrm{~m}^{2} \mathrm{~h}^{-1}$ or less as it is proven that it produces low backpressures and good uptakes of $\mathrm{Cu}^{2+}$ under the studied conditions.

- Use the cartridge for concentrations below $7.9 \mathrm{mmol} \mathrm{L}^{-1}$ of $\mathrm{Cu}^{2+}$.

- The $\mathrm{pH}$ of the solution should be greater than 3, a value of 4 is preferred.

- Keep the outlet of the RFC far away of the inlet in order increase the probability of recirculating contaminated solution. 


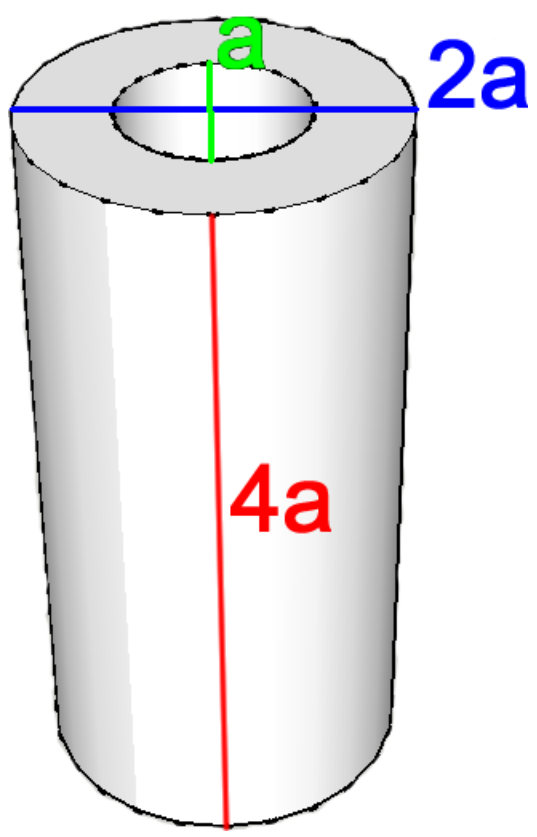

Figure 5.16: Ratio of the inner feed cylinder diameter to the sorbent holder diameter to the cylinder to the length. The ratio is a:2a:4a.

In order to estimate the size the RFC it is necessary to know the volume between the two cylinders. This parameter will help to estimate the volume of NCaSil that needs to be placed inside the device. Therefore it is necessary to subtract the volume of the inner feed cylinder to the volume of the sorbent holder cylinder as shown in Eq. 5.8.

$$
V_{R F C}=\frac{\pi}{4}(2 a)^{2} \cdot 4 a-\frac{\pi}{4}(a)^{2} \cdot 4 a=3 \pi a^{3}
$$

Where $V_{R F C}$ is the volume between the two cylinders which is packed with NCaSil and $a$ is a length which arises from the ratio of $D_{\text {int }}: D_{S B} C_{L}=a: 2 a: 4 a$ (refer to Figure 5.16). The mass of NCaSil necessary to fill this volume is obtained multiplying $V_{R F C}$ by $\rho_{\mathrm{NCaSil}}$ as shown in Eq. 5.9.

$$
\left(3 \pi \cdot a^{3}\right) \cdot \rho_{N C a S i l}=m_{N C a S i l}
$$

Moreover, in order to be able to obtain the mass of NCaSil needed to treat a solution of any given concentration it is important to know the volume of solution to be treated (a rough estimation may be made from the size of the tank) and the concentration of $\mathrm{Cu}^{2+}$ in solution. This is represented in Eq. 5.10.

$$
\frac{V_{\text {treated }} \cdot\left[\mathrm{Cu}^{2+}\right]}{q_{m}}=m_{\text {NCaSil }}
$$

Where $m_{\mathrm{NCaSil}}$ is the minimum mass of NCaSil needed treat the solution. It is noteworthy that is $\rho_{N C a S i l}$ which converts the mass of NCaSil into a volume, establishing a relation between $a$ and the other variables. 
Knowing that both terms in Eq. 5.9 and Eq. 5.10 are equal to the necessary mass of NCaSil, it is possible to combine both equations to obtain equation 5.11 .

$$
\left(3 \pi a^{3}\right) \cdot \rho_{\text {NCaSil }}=\frac{V_{\text {treated }} \cdot\left[\mathrm{Cu}^{2+}\right]}{q_{m}}
$$

Rearranging Eq. 5.11 it is possible to obtain the value of $a$ for the RFC of a ratio $a: 2 a: 4 a$ used in this study:

$$
a=\left(\frac{V_{\text {treated }} \cdot\left[\mathrm{Cu}^{2+}\right]}{q_{m}} \cdot \frac{1}{\rho_{\text {NCaSil }} \cdot 3 \pi}\right)^{1 / 3}
$$

Where $a$ is the calculated length regarding the $a: 2 a: 4 a$ ratio in metres (refer to Figure 5.16), $V_{\text {treated }}$ is the volume to be treated in $\mathrm{m}^{3},\left[\mathrm{Cu}^{2+}\right]$ is the initial concentration of copper in solution in mol m $\mathrm{m}^{-3}, q_{m}$ is the maximum adsorption capacity of NCaSil for copper $7 \mathrm{~mol} \mathrm{~kg}^{-1}$ (underestimated on purpose) and $\rho_{\text {NCaSil }}$ is the bulk density of NCaSil equal to $70 \mathrm{~kg} \mathrm{~m}^{-3}$.

If $a$ is larger than $0.5 \mathrm{~m}$ it will make the cost of designing the RFC too expensive, therefore it is recommended to divide it into several smaller columns. For example, if the volume to be treated is $10 \mathrm{~m}^{3}$ with a concentration of $7.9 \mathrm{~mol} \mathrm{~m}^{-3}$, therefore a value of $a \approx 1 \mathrm{~m}$ is obtained from Eq. 5.12 which in turn makes the RFC 4 metres in height with an external diameter of 2 metres. Hence it is recommended that in such cases 5 smaller RFC should be built with a value of $a$ equal to 0.2 metre in this specific case. This will also allow a distribution of devices in the tank enabling a more even treatment of the solution. Values of $a$ larger than $0.2 \mathrm{~m}$ will probably result in significant costs due to the specifications of the materials needed to build a RFC device. In terms of cost considerations it is recommended that the value of $a$ should be less than $0.2 \mathrm{~m}$.

The cross sectional area may be obtained from Eq. 5.13 for an RFC following $a: 2 a: 4 a$ ratio;

$$
A_{\text {cross,sec }}=\frac{4 \cdot \pi \cdot a^{2}}{\ln (2)}
$$

In addition, the hydraulic loading for this specific device is represented by the following equation:

$$
H=\frac{Q \cdot \ln (2)}{4 \cdot \pi \cdot a^{2}}
$$


Then considering that in this study a hydraulic loading of $1.6 \mathrm{~m}^{3} \mathrm{~m}^{2} \mathrm{~h}^{-1}$ was used the relationship between the flow to be used and parameter $a$ is represented in Eq. 5.15:

$Q=\frac{6.4 \cdot \pi \cdot a^{2}}{\ln (2)}$

$Q$ is the flow in $\mathrm{m}^{3} \mathrm{~h}^{-1}$, the value 6.4 is a dimensional factor in $\mathrm{m}^{3} \mathrm{~m}^{-2} \mathrm{~h}^{-1}$ and $a$ the length in $\mathrm{m}$ obtained from $\mathrm{Eq} 5.12$.

It is recommended to keep a value of $\mathrm{Q}$ within $\pm 10 \%$ since this flow is proven to work well.

The RFC specifications regarding the volume and concentration of $\mathrm{Cu}^{2+}$ to be treated are summarized in Table 5.2. 
Table 5.2: Radial flow column specifications regarding the volume of solution to be treated and the concentration of $\mathrm{Cu}^{2+}$ in solution.

\begin{tabular}{|c|c|c|c|c|c|c|c|c|}
\hline \multicolumn{2}{|c|}{ Operational parameters } & \multicolumn{7}{|c|}{ RFC specifications } \\
\hline $\begin{array}{c}\text { Volume of the } \\
\text { solution to be treated } \\
{\left[\mathrm{m}^{3}\right]}\end{array}$ & $\begin{array}{l}\mathrm{Cu}^{2+} \text { conc. } \\
{\left[\mathrm{mol} \mathrm{m}^{-3}\right]}\end{array}$ & $\begin{array}{l}\text { Calculated } \\
\text { value of } a \\
{[\mathrm{~m}]}\end{array}$ & $\underset{\left[\mathrm{m}^{3} \mathrm{~h}^{-1}\right]}{Q}$ & $\begin{array}{c}\text { Ethanol washed } \\
\text { NCaSil dry } \\
\text { mass, } m_{N C a S i l} \\
{[\mathrm{~kg}]}\end{array}$ & $\begin{array}{l}\text { Number of } \\
\text { RFC to be } \\
\text { constructed }\end{array}$ & $\begin{array}{c}\text { Internal } \\
\text { diameter } \\
D_{i n t} \\
{[\mathrm{~cm}]}\end{array}$ & $\begin{array}{c}\text { External } \\
\text { diameter } \\
D_{S B} \\
{[\mathrm{~cm}]}\end{array}$ & $\begin{array}{c}\text { Length } \\
C_{L} \\
{[\mathrm{~cm}]}\end{array}$ \\
\hline 1 & 1 & 0.06 & 0.1 & 0.1 & 1 & 6 & 12 & 24 \\
\hline 1 & 3 & 0.09 & 0.2 & 0.4 & 1 & 9 & 18 & 36 \\
\hline 1 & 6 & 0.11 & 0.3 & 1 & 1 & 11 & 22 & 44 \\
\hline 5 & 1 & 0.10 & 0.3 & 1 & 1 & 10 & 20 & 40 \\
\hline 5 & 3 & 0.15 & 0.6 & 2 & 1 & 15 & 30 & 59 \\
\hline 5 & 6 & 0.19 & 1.0 & 4 & 1 & 19 & 38 & 76 \\
\hline 10 & 1 & 0.13 & 0.5 & 1 & 1 & 13 & 26 & 52 \\
\hline 10 & 3 & 0.19 & 1.0 & 4 & 1 & 19 & 38 & 76 \\
\hline 10 & 6 & 0.24 & 1.6 & 9 & 2 & 12 & 24 & 48 \\
\hline 50 & 1 & 0.22 & 1.4 & 7 & 2 & 11 & 22 & 44 \\
\hline 50 & 3 & 0.32 & 3.0 & 21 & 2 & 16 & 32 & 64 \\
\hline 50 & 6 & 0.40 & 4.7 & 43 & 2 & 20 & 40 & 80 \\
\hline 100 & 1 & 0.28 & 2.3 & 14 & 2 & 14 & 28 & 56 \\
\hline 100 & 3 & 0.40 & 4.7 & 43 & 2 & 20 & 40 & 80 \\
\hline 100 & 6 & 0.51 & 7.4 & 86 & 3 & 17 & 34 & 68 \\
\hline
\end{tabular}

Values of $m_{N C a S i l}, a$ and $Q$ were calculated using equations 5.10, 5.12 and 5.15, respectively. Values of $a$ exceeding 0.2 metres were divided into smaller columns as detailed on Table 5.2. Please refer to Figure 5.16 regarding the ratio of $\operatorname{Dint}: D_{S B}: C_{L}=a: 2 a: 4 a$. 


\subsection{Chapter Conclusions}

The removal of $\mathrm{Cu}^{2+}$ ions from solution using a conventional axial flow column proved to be unpractical. High pressure drops, greater than $20 \mathrm{kPa}$ per $\mathrm{cm}$ of bed, were generated when a bed height to column diameter ratio of 2.5:1 was used. Furthermore, the pressure drop varied with time, attributable to changes in the particle size diameter, particle shape and void spaces. These pressure drop variations most likely arose from void spaces blockage. Blockage occurred due to structural changes in $\mathrm{NCaSil}$ as $\mathrm{Ca}^{2+}$ and silica were released and due to the formation of amorphous copper hydroxide species on the surfaces of NCaSil. In fact, void space blockage was a serious factor at fairly low $\mathrm{Cu}^{2+}$ concentrations of $1.6 \mathrm{mmol} \mathrm{L}^{-1}$ mainly due to copper hydroxide forming on the surface. At higher concentrations of 7.9 and $15.7 \mathrm{mmol} \mathrm{L}^{-1}$ the pressure drop rose during the first portion of the experiment but then declined consistent with crystalline copper hydroxide sulfate species forming on the surface of NCaSil, which allowed stable channels and void spaces to form. Additionally, high concentrations of $\mathrm{Cu}^{2+}$ in the feed solution resulted in early breakthrough in all columns.

Using a radial flow column was an improvement over a standard axial flow column since the pressure drop was below $10 \mathrm{kPa}$. A clear breakthrough point was observed at low concentrations of $1.6 \mathrm{mmol} \mathrm{L}^{-1}$ of $\mathrm{Cu}^{2+}$. At higher concentrations of 7.9 and $15.7 \mathrm{mmol} \mathrm{L}^{-1}$ the breakthrough appeared almost instantaneously.

Overall, the operation of an axial and radial flow column in a continuous arrangement was an improvement from previous attempts since the concentration at the outlet was kept below 3\% for short period of times. Nonetheless, the use of the axial flow column was not practical due to large pressure drops and changing particle morphology which led to free channel formations and early breakthroughs.

To explore better operational conditions the RFC was used by immersing the device in a tank and recirculating the solution through the device with outward flow. The results were very good, behaving in a similar manner to batch reactors. Nonetheless, the time to reach equilibrium were higher than in batch experiments with a value 180 and $360 \mathrm{~min}$ for 1.6 and $7.9 \mathrm{mmol} \mathrm{L}^{-1} \mathrm{Cu}^{2+}$ solutions, respectively. Therefore it is recommended that it is used for low concentration range in order to remain 
competitive with batch reactors. Although longer contact times are required there is no need for a filtration step, thus saving costs in filtration time and equipment.

Scale-up equations were presented where the key factor was to keep constant the ratio of feed cylinder diameter $\left(D_{\text {int }}\right)$ to the sorbent holder diameter $\left(D_{S B}\right)$ to the cartridge length $\left(C_{L}\right)$ in the following form $D_{\text {int }}: D_{S B}: C_{L}=a: 2 a: 4 a$ during the scale-up. The value of $a$ should remain below $0.2 \mathrm{~m}$ in order to avoid expensive raw materials in the construction of the RFC. If the value of $a$ is higher than 0.2 then it should be split into two or more smaller RFCs to reduce cost. 


\section{Chapter 6, Thesis Conclusions}

The uptake of $\mathrm{Cu}^{2+}$ by NCaSil, using a copper sulfate solution, is endothermic. An increase in entropy of the system displaces the equilibrium towards the reagents, thus the uptake is becoming more spontaneous when the temperature increases. From a kinetic point view the reaction is also affected in a large extent by the change in temperature, with an activation energy of $54 \mathrm{~kJ} \mathrm{~mol}^{-1}$, indicating a chemical reaction between $\mathrm{Cu}^{2+}$ and the surface of NCaSil.

The uptake mechanism of copper onto NCaSil may be summarized in the following simplified steps. First, NCaSil in water rapidly gains a negative charge on the surface due to the leaching of calcium. This creates an electrostatic attraction between the surface of $\mathrm{NCaSil}$ and $\mathrm{Cu}^{2+}$ present in solution aiding the movement of $\mathrm{Cu}^{2+}$ towards the surface. Second, $\mathrm{Cu}^{2+}$ ions chemically react with the surface forming insoluble amorphous copper hydroxide. This statement is supported by the literature and this study, where non crystalline copper containing regions on the surface of NCaSil could be observed by SEM-EDS. Third, depending on the temperature and concentration amorphous copper hydroxide is transformed via nucleation of sulfate into copper sulfate hydroxide minerals. At low temperatures $(\approx 293 \mathrm{~K})$ and concentrations of $\mathrm{Cu}^{2+}\left(<7.9 \mathrm{mmol} \mathrm{L}^{-1}\right)$, wroewolfeite and posnjakite are the preferred structures, while at high concentrations $\left(15.7 \mathrm{mmol} \mathrm{L}^{-1}\right)$ and temperatures $(333 \mathrm{~K})$ the formation of brochantite is preferred. Furthermore, at a concentration of $15.7 \mathrm{mmol} \mathrm{L}^{-1}$ of $\mathrm{Cu}^{2+}$ and at STP conditions ( $1 \mathrm{~atm}$ and $293 \mathrm{~K}$ ) wroewolfeite and posnjakite are intermediates in the formation of the thermodynamically stable product brochantite.

NCaSil releases calcium and silicate into solution, promoted by increased temperature. Hence, even though NCaSil exhibits a high loading capacity and fast 
kinetics, it is only recommended for industrial applications where silica and calcium carbonate scaling may not be an issue.

A kinetic follow-up of the crystal growth of these 3 copper sulfate hydroxide minerals was done using area of the reflections of powder-XRD patterns gathered over time. It was found that the rate determining step in the formation of brochantite is the nucleation of sulfate in the crystal lattice. This is supported by the fact that sulfate related reflections were the ones which exhibited the slowest initial growth rates. Additionally the activation energy of the crystallographic plane 420 of brochantite was calculated to be $42 \mathrm{~kJ} \mathrm{~mol}^{-1}$.

The $\mathrm{pH}$ of the solution was found to be important for the application for the treatment of mining waste waters. Often these have very low $\mathrm{pH}$ values, which would need to be adjusted to a value greater than 3 to make use of NCaSil feasible. The treatment of an emulated mining waste was successful after the $\mathrm{pH}$ was adjusted. Copper, lead and iron ions were removed readily from solution. A continuous process production of $\mathrm{NCaSil}$ could be coupled to the existing processes in the market.

To develop a practical operation model for an industrial adsorption of $\mathrm{Cu}^{2+}$ by NCaSil several types of column filtration were investigated. A radial flow column design was chosen considering the kinetic and equilibrium properties of the material. In prior studies it was shown that NCaSil released calcium to the solution, which it was not unexpected since ion exchange resins and aluminosilicate materials exchange ions during the uptake of metals from solution. The problem arises when the morphology of NCaSil changes as calcium and monomeric silica is leached into solution. This changes the void space between the aggregates and in some cases even blocks them due to the formation of amorphous copper hydroxide. These observations contrast with other adsorbents where their structure remains apparently unchanged during a column and batch operation. What's more, low concentrations of copper in the feed solution generate a copper hydroxide film drastically increasing the pressure drop through the column. Hence, the operation using conventional axial flow columns or radial flow column proved to be unpractical. The former generates large pressure drops, while the latter presents short operational times.

The attempt to operate the radial flow column by immersing the device in a tank containing a copper solution and recirculating it through the device was promising. The operation showed similar behaviour to the one observed in the batch process. 
Furthermore, it is an improvement over batch reactors since there is no need for filtration. Equations related to the scale-up were presented, where the ratio of the device employed in this thesis was kept constant and showing a feasible route for employing NCaSil on an industrial scale in the remediation of copper waste waters.

Finally, if NCaSil is intended to be applied to uptake metals ions from solution on a large scale it is recommended to use it either in batch mode or in a semi-batch mode by immersion of a RFC packed with $\mathrm{NCaSil}$ in a tank containing a $\mathrm{Cu}^{2+}$ solution. 


\section{Future work}

The application of the RFC via immersion for the uptake of phosphate should be studied since no filtration is needed when this device is used. In a similar manner the RFC should be tested to treat real AMD or weak acid produced during mining operations.

Moreover, the formation of brochantite, as a result of $\mathrm{Cu}^{2+}$ uptake by NCaSil, was found to have an application as a fungicide in the agrochemical industry. Standard methods of preparation of these minerals use ten times more copper to produce a mixture of copper sulfate hydroxide compounds by addition of lime into a copper sulfate solution. Additionally, the resulting product has a high content of bassanite, calcium sulfate hemi hydrate, which block the nozzles of the sprayers. Therefore this study offers a competitive method for the production of bassanite-free copper based fungicide. Therefore market research should be undertaken to analyse the necessity of this improved product.

On a different aspect, the copper sulfate hydroxide minerals synthetized in this study may be transformed into catalytically active compounds. It is well known that copper (I) and copper (II) oxide (tenorite) are catalytically active [119-121]. The former is known to be photochemically active while the latter may catalyse the reduction of isopropanol into propene. Additionally, the formation of tenorite sub-micron particles by thermal decomposition of brochantite crystals has been recently reported [120]. Following the advice of the author of this thesis, exploratory experiments have been conducted by Mahroo Fallah at the McDiarmid Institute, to incorporate brochantite crystals synthetized in this study, into a geopolymer matrix. Crystals will decompose into tenorite when heated. First releasing water, through the condensation of hydroxyl groups, followed by the release $\mathrm{SO}_{2}$ at higher temperature ( $\approx 973 \mathrm{~K}$ ). Subsequently void spaces will be generated creating a porous material. The first 
attempt resulted in tenorite particles ranging from 50 to $200 \mathrm{~nm}$, but the surface area was low approximately $10 \mathrm{~m}^{2} \mathrm{~g}^{-1}$. This value is 5 to 10 times less than the reported surface area of commercially available silica-supported tenorite. Nonetheless, particles didn't agglomerate. This was an improvement over previous attempts where the addition of copper oxide nanoparticles into the geopolymer matrix resulted in serious agglomeration. An SEM image in normal and backscatter mode is presented in appendix A-22. 


\section{References}

1. C. N. Mulligan, R. N. Yong, and B. F. Gibbs, Remediation technologies for metal-contaminated soils and groundwater: an evaluation. Engineering Geology, 2001. 60(1-4): p. 193-207.

2. G. McKay, Use of adsorbents for the removal of pollutants from wastewaters, 1996, CRC Press.

3. Y. S. Ho, J. C. Y. Ng, and G. Mckay, Kinetics of pollutant sorption by biosorbents: Review. Separation and Purification Methods, 2000. 29(2): p. 189-232.

4. Y. S. Ho and G. McKay, Sorption of dyes and copper ions onto biosorbents. Process Biochemistry, 2003. 38(7): p. 1047-1061.

5. S. Chakravarty, S. Pimple, H. T. Chaturvedi, S. Singh, and K. K. Gupta, Removal of copper from aqueous solution using newspaper pulp as an adsorbent. Journal of Hazardous Materials, 2008. 159(2-3): p. 396-403.

6. Nalco, The Nalco Water Handbook. Second Edition ed, 1988, McGraw-Hill Inc.

7. M. A. Alghoul, P. Poovanaesvaran, K. Sopian, and M. Y. Sulaiman, Review of brackish water reverse osmosis (BWRO) system designs. Renewable \& Sustainable Energy Reviews, 2009. 13(9): p. 2661-2667.

8. Precipitaciones. 2009, Available from: http://www.meteochile.cl/precipitacion.html.

9. M. Valko, Metals, toxicity and oxidative stress. Current Medicinal Chemistry, 2005. 12(10): p. 1161-1208. 
10. D. W. Boening, Ecological effects, transport, and fate of mercury: a general review. Chemosphere, 2000. 40(12): p. 1335-1351.

11. L. M. Gaetke, C. K Chow, Copper toxicity, oxidative stress, and antioxidant nutrients. Toxicology, 2003. 189: p. 147-163.

12. T. D. Rae, P. J. Schmidt, R. A. Pufahl, V. C. Culotta, and T. V. O'Halloran, Undetectable Free Intracellular Copper: the Requirement of a Copper Chaperone for Superoxide Dismutase. Science, 1999. 284: p. 805-808.

13. S. I. Dikalov, M. P. Vitek, R. P. Mason, Cupric-amyloid $\beta$ peptide complex stimulates oxidation of ascorbate and generation of hydroxyl radical. Free Radical Biology and Medicine, 2004. 36(3): p. 340-347.

14. CONAMA, Decreto Supremo $n^{\circ} 90$, cited 2011, Available from: http://www.sinia.cl/1292/articles-27153_recurso_1.pdf.

15. Wellington trade waste bylaws, cited 2011, Available from: http://www.wellington.govt.nz/plans/bylaws/tradewaste09.html.

16. W. S. Ngah, Adsorption of copper on rubber (Hevea brasiliensis) leaf powder: Kinetic, equilibrium and thermodynamic studies. Biochemical Engineering Journal, 2008. 39(3): p. 521-530.

17. H. Runping, Characterization of modified wheat straw, kinetic and equilibrium study about copper ion and methylene blue adsorption in batch mode. Carbohydrate Polymers, 2010. 79(4): p. 1140-1149.

18. R. A. Rao, Biosorption of bivalent metal ions from aqueous solution by an agricultural waste: Kinetics, thermodynamics and environmental effects. Colloids and Surfaces a-Physicochemical and Engineering Aspects, 2009. 332(2-3): p. 121-128.

19. R. Djeribi, Sorption of copper(II) from aqueous solutions by cedar sawdust and crushed brick. Desalination, 2008. 225(1-3): p. 95-112.

20. Y. K. Bayhan and N. Ertugay, The removal of copper (II) ion by using mushroom biomass (Agaricus bisporus) and kinetic modelling. Desalination, 2010. 255(1-3): p. 137-142.

21. E. Erdem, N. Karapinar, and R. Donat, The removal of heavy metal cations by natural zeolites. Journal of Colloid and Interface Science, 2004. 280(2): p. 309-314. 
22. S. Babel and T. A. Kurniawan, Low-cost adsorbents for heavy metals uptake from contaminated water: a review. Journal of Hazardous Materials, 2003. 97(1-3): p. 219-243.

23. G. Barassi, A. Valdes, C. Araneda, C. Basualto, J. Sapag, C. Tapia, and F. Valenzuela, $\mathrm{Cr}(\mathrm{VI})$ sorption behavior from aqueous solutions onto polymeric microcapsules containing a long-chain quaternary ammonium salt: Kinetics and thermodynamics analysis. Journal of Hazardous Materials, 2009. 172(1): p. 262-268.

24. E. Guibal, Interactions of metal ions with chitosan-based sorbents: a review. Separation and Purification Technology, 2004. 38(1): p. 43-74.

25. M. I. Panayotova, Kinetics and thermodynamics of copper ions removal from wastewater by use of zeolite. Waste Management, 2001. 21(7): p. 671-676.

26. E. Pehlivan and T. Altun, The study of various parameters affecting the ion exchange of $\mathrm{Cu}^{2+}, \mathrm{Zn}^{2+}, \mathrm{Ni}^{2+}, \mathrm{Cd}^{2+}$, and $\mathrm{Pb}^{2+}$ from aqueous solution on Dowex 50W synthetic resin. Journal of Hazardous Materials, 2006. 134(1-3): p. 149-156.

27. E. Pehlivan and T. Altun, Ion-exchange of $\mathrm{Pb}^{2+}, \mathrm{Cu}^{2+}, \mathrm{Zn}^{2+}, \mathrm{Cd}^{2+}$, and $\mathrm{Ni}^{2+}$ ions from aqueous solution by Lewatit CNP 80. Journal of Hazardous Materials, 2007. 140(1-2): p. 299-307.

28. E. Pehlivan, A. M. Ozkan, S. Dinc, and S. Parlayici, Adsorption of $\mathrm{Cu}^{2+}$ and $\mathrm{Pb}^{2+}$ ion on dolomite powder. Journal of Hazardous Materials, 2009. 167(13): p. 1044-1049.

29. X. S. Wang and Y. Qin, Equilibrium sorption isotherms for of $\mathrm{Cu}^{2+}$ on rice bran. Process Biochemistry, 2005. 40(2): p. 677-680.

30. G. Blanchard, Removal of heavy metals from waters by means of natural zeolites. Water Research, 1984. 18: p. 1501-1507.

31. N. K. Hamadi, X. D. Chen, M. M. Farid, and M. G. Q. Lu, Adsorption kinetics for the removal of chromium(VI) from aqueous solution by adsorbents derived from used tyres and sawdust. Chemical Engineering Journal, 2001. 84(2): p. 95-105.

32. E. Pehlivan and S. Cetin, Sorption of Cr(VI) ions on two Lewatit-anion exchange resins and their quantitative determination using $U V$-visible spectrophotometer. Journal of Hazardous Materials, 2009. 163(1): p. 448453. 
33. F. Gode and E. Pehlivan, Removal of chromium(III) from aqueous solutions using Lewatit S 100: The effect of $\mathrm{pH}$, time, metal concentration and temperature. Journal of Hazardous Materials, 2006. 136(2): p. 330-337.

34. Y. T. Zhou, H. L. Nie, C. Branford-White, Z. Y. He, and L. M. Zhu, Removal of $\mathrm{Cu}^{2+}$ from aqueous solution by chitosan-coated magnetic nanoparticles modified with alpha-ketoglutaric acid. Journal of Colloid and Interface Science, 2009. 330(1): p. 29-37.

35. J. H. Johnston, A. J. McFarlane, T. Borrmann, and J. Moraes, Nanostructured silicas and silicates - new materials and their applications in paper. Current Applied Physics, 2004. 4(2-4): p. 411-414.

36. Andrew James McFarlane, The Synthesis and Characterisation of NanoStructured Calcium Silicate, Ph.D., 2007, Faculty of Chemical and Physical Sciences, Victoria University of Wellington.

37. Mathew James Cairns, A Study of the Sorption Characteristics of Nanostructured Calcium SIlicate, Ph.D., 2008, Faculty of Chemical and Physical Sciences, Victoria University of Wellington.

38. T. Borrmann, Calcium Silicate Facilitated Polymerisation of MAS, 2006. ICSM The International Conference on Science and Technology of Synthetic Metals, Trinity College, Dublin, Ireland.

39. D. C. Southam, T. W. Lewis, A. J. McFarlane, and J. H. Johnston, Amorphous calcium silicate as a chemisorbent for phosphate. Current Applied Physics, 2004. 4(2-4): p. 355-358.

40. M. J. Cairns, T. Borrmann, W. H. Holl, and J. H. Johnston, A study of the uptake of copper ions by nanostructured calcium silicate. Microporous and Mesoporous Materials, 2006. 95(1-3): p. 126-134.

41. K. Xia, A. Mehadi, R. W. Taylor, and W. F. Bleam, X-ray absorption and electron paramagnetic resonance studies of $\mathrm{Cu}(\mathrm{II})$ sorbed to silica: Surfaceinduced precipitation at low surface coverages. Journal of Colloid and Interface Science, 1997. 185(1): p. 252-257.

42. P. A. O'Day, C. J. Chisholm-Brause, S. N. Towle, G. A. Parks, and G. E. Brown, X-ray absorption spectroscopy of Co(II) sorption complexes on quartz (alpha-SiO 2$)$ and rutile $\left(\mathrm{TiO}_{2}\right)$. Geochimica et Cosmochimica Acta, 1996. 60(14): p. 2515-2532.

43. K. G. Scheckel and D. L. Sparks, Temperature effects on nickel sorption kinetics at the mineral-water interface. Soil Science Society of America Journal, 2001. 65(3): p. 719-728. 
44. K. M. Spark, B. B. Johnson, and J. D. Wells, Is Copper(II) Hydroxide an Intermediate in the Formation of the Hydroxide Chloride and Hydroxide Nitrate of Copper(II). Australian Journal of Chemistry, 1990. 43(4): p. 749754.

45. M. Klimsa, 2013, Ph.D., School of Chemical and Physical Sciences, Victoria University of Wellington.

46. A. Akcil, Acid Mine Drainage (AMD): causes, treatment and case studies. Journal of Cleaner Production, 2006. 14(12-13): p. 1139-1145.

47. B. Christensen, M. Laake, and T. Lien, Treatment of acid mine water by sulfate-reducing bacteria; Results from a bench scale experiment. Water Research, 1996. 30(7): p. 1617-1624.

48. K. Sutherland, Filters and Filtration Handbook. 5th ed, 2008, Elsevier.

49. R.W. Kuennen, Carbon block water filter, 2002, USPTO, United States of America.

50. B. Sivasankar, Bioseparations: Principles And Techniques, 2006, PrenticeHall of India.

51. M. A. Desai, Downstream Processing of Proteins: Methods and Protocols, 2000, Humana Press.

52. A. S. Rathore and A. Velayudhan, Scale-up and Optimization in Preparative Chromatography: Principles and Biopharmaceutical Applications, 2002, Marcel Dekker Incorporated.

53. Ralph K. Iler, The chemistry of silica: solubility, polymerization, colloid and surface properties, and biochemistry, 1979, Wiley.

54. P. Konieczka and J. Namiesnik, Quality assurance and quality control in the analytical chemical laboratory : a practical approach, 2009, CRC Press.

55. Shimadzu Tokyo, Estimation of uncertainty in flame atomic absorption spectrophotometry, cited 2012, Available from: http://www2.shimadzu.com/apps/appnotes/app312.pdf

56. S. Lagergren, Zur theorie der sogenannten adsorption gelöster stoffe. K. Sven. Vetenskapsakad. Hand1, 1898. 24: p. 1-39. 
57. Y. S. Ho, Review of second-order models for adsorption systems. Journal of Hazardous Materials, 2006. 136(3): p. 681-689.

58. R. S. Juang and M. L. Chen, Application of the Elovich equation to the kinetics of metal sorption with solvent-impregnated resins. Industrial \& Engineering Chemistry Research, 1997. 36(3): p. 813-820.

59. Y. S. Ho and G. McKay, Pseudo-second order model for sorption processes. Process Biochemistry, 1999. 34(5): p. 451-465.

60. C. S. Zhu, L. P. Wang, and W. B. Chen, Removal of Cu(II) from aqueous solution by agricultural by-product: Peanut hull. Journal of Hazardous Materials, 2009. 168(2-3): p. 739-746.

61. M. E. Argun, S. Dursun, A new approach to modification of natural adsorbent for heavy metal adsorption. Bioresource Technology, 2008. 99(7): p. 2516-2527.

62. K. Oldham, J. Myland, and A. Bond, Electrochemical Science and Technology: Fundamentals and Applications, 2011, John Wiley \& Sons.

63. G. L. Dotto and L. A. A. Pinto, Adsorption of food dyes acid blue 9 and food yellow 3 onto chitosan: Stirring rate effect in kinetics and mechanism. Journal of Hazardous Materials, 2011. 187(1-3): p. 164-170.

64. R. Apiratikul and P. Pavasant, Sorption of $\mathrm{Cu}^{2+}, \mathrm{Cd}^{2+}$, and $\mathrm{Pb}^{2+}$ using modified zeolite from coal fly ash. Chemical Engineering Journal, 2008. 144(2): p. 245-258.

65. S. Arrhenius, Ober die Reacktionsgeschwindigkeit bei der Inversion Rohrzucker durch Saüren. Z. Physik. Chem., 1889. 4: p. 226-248.

66. M. E. Argun and S. Dursun, A new approach to modification of natural adsorbent for heavy metal adsorption. Bioresource Technology, 2008. 99(7): p. 2516-2527.

67. R. K. Iler, The chemistry of silica : solubility, polymerization, colloid and surface properties, and biochemistry, 1979, Wiley.

68. Y. Kuroda, A. Kotani, H. Maeda, H. Moriwaki, T. Morimato, and M. Nagao, The State of Excessively Ion-Exchanged Copper in Mordenite - Formation of Tetragonal Hydroxy-Bridged Copper-Ion. Journal of the Chemical SocietyFaraday Transactions, 1992. 88(11): p. 1583-1590.

69. Mindat.org. Wroewolfeite, cited 2012, Available from: http://www.mindat.org/min-4315.html. 
70. Mindat.org. Posnjakite, cited http://www.mindat.org/min-3265.html.

71. Mindat.org, Brochantite, cited 2012, Available from: http://www.mindat.org/min-779.html.

72. C. Y. Tang, V. T. Do, M. Reinhard, and J. O. Leckie, Degradation of polyamide nanofiltration and reverse osmosis membranes by hypochlorite. Environmental Science \& Technology, 2012. 46: p. 852-859.

73. M. K. Wittholz, B. K. O'Neill, C. B. Colby, and D. Lewis, Estimating the cost of desalination plants using a cost database. Desalination, 2008. 229: p. 10-20.

74. T. R. Dabinett, D. Humberstone, P. Leverett, and P. A. Williams, Synthesis and stability of wroewolfeite, $\mathrm{Cu}_{4} \mathrm{SO}_{4}(\mathrm{OH})_{6} \cdot 2 \mathrm{H}_{2} \mathrm{O}$. Pure and Applied Chemistry, 2008. 80(6): p. 1317-1323.

75. R. Kas and O. Birer, Sonochemical shape control of copper hydroxysulfates. Ultrasonics Sonochemistry, 2012. 19(3): p. 692-700.

76. J. Huato, T. Ogura, Method for making colloidal cupric compounds and their uses, US 2002/01367781 A1, 2002, USPTO, United States of America.

77. M. Courtade, G. Ramel, Bordeaux mixture, process for its manufacture and cupric fungicidal compositions containing it, 5,958,438, 1999, USPTO, United States of America.

78. F. Ferrier, G. Joncheray, M. Pillot, Method of producing copper hydoroxosulphates and copper fungicidal compositions containing same, US 2007/0003635 A1, 2007, USPTO, United States of America.

79. C. H. Rogers, Method of preparing Bordeaux Mixture Concentrate, 2,998,345, 1961, USPTO, United States of America.

80. J. Pickworth Glusker and K. N. Trueblood, Crystal structure analysis : a primer. 2nd ed, 1985, Oxford University Press.

81. S. Merlino, N. Perchiazzi, and D. Franco, Brochantite, Cu4SO4(OH)(6): OD character; polytypism and crystal structures. European Journal of Mineralogy, 2003. 15(2): p. 267-275.

82. I. Langmuir, The Adsorption of Gases on Plane Surfaces of Glass, Mica and Platinum. Journal of the American Chemical Society, 1918. 40(9): p. 13611403. 
83. H. Freundlich, Grundzüge derkollodlehere, 1925, London, Methuen.

84. A. W. Adamson, Physical Chemistry of Surfaces. 2nd ed, 1967, John Wiley \& Inc.

85. M. A. M. Lawrence, N. A. Davies, P. A. Edwards, M. G. Taylor, and K. Simkiss, Can adsorption isotherms predict sediment bioavailability? Chemosphere, 2000. 41(7): p. 1091-1100.

86. P. W. Atkins, Physical Chemistry, 1990, Oxford University Press.

87. M. A. Sanchez. Proceedings of the IV International Conference on Clean Technologies for the Mining Industry. in Environment \& innovation in mining and mineral technology. 1998. Santiago, Chile: University of Concepción.

88. P. Ravenscroft, H. Brammer, and K. Richards, Arsenic Pollution: A Global Synthesis, 2011, John Wiley \& Sons.

89. L. M. Toovey, The Top 10 Copper Producing Countries. 2009, Available from: http://copperinvestingnews.com/4147/the-top-10-copper-producingcountries/.

90. J. Newbold, Chile's environmental momentum: ISO 14001 and the largescale mining industry - Case studies from the state and private sector. Journal of Cleaner Production, 2006. 14(3-4): p. 248-261.

91. A. Black, D. Craw, J. H. Youngson, and J. Karubaba, Natural recovery rates of a river system impacted by mine tailing discharge: Shag River, East Otago, New Zealand. Journal of Geochemical Exploration, 2004. 84(1): p. 21-34.

92. N. J. Wilson, Craw, D., Hunter, K., Contributions of discharges from a historic antimony mine to metalloid content of river waters, Marlborough, New Zealand. Journal of Geochemical Exploration, 2004. 84(3): p. 127-139.

93. P. A. Weber, W. Skinner, J. B. Hughes, P. Lindsay, and T. A. Moore, Source of $\mathrm{Ni}$ in coal mine acid rock drainage, West Coast, New Zealand. International Journal of Coal Geology, 2006. 67(4): p. 214-220.

94. L. Haffert, Processes of attenuation of dissolved arsenic downstream from historic gold mine sites, New Zealand. Science of the Total Environment, 2008. 405(1-3): p. 286-300. 
95. M. Ramirez, S. Massolo, R. Frache, and J. A. Correa, Metal speciation and environmental impact on sandy beaches due to El Salvador copper mine, Chile. Marine Pollution Bulletin, 2005. 50(1): p. 62-72.

96. R. G. Trucco, Heavy-Metal Concentration in Sediments from Tongoy and Herradura Bays, Coquimbo, Chile. Marine Pollution Bulletin, 1990. 21(5): p. 229-232.

97. Environmental Protection Agency U.S. Technical Document, Acid Mine Drainage Prediction, 1994, Available from: http://www.epa.gov/epawaste/nonhaz/industrial/special/mining/techdocs/amd .pdf.

98. F. Valenzuela, J. Cabrera, C. Basualto, and J. Sapag-Hagar, Kinetics of copper removal from acidic mine drainage by a liquid emulsion membrane. Minerals Engineering, 2005. 18(13-14): p. 1224-1232.

99. B. Aubé. The Science of Treating Acid Mine Drainage and Smelter Effluents. Available from: http://www.enviraube.com/tech/treatment_science.pdf.

100. M. E. Schlesinger, M. E. Davenport, M. J. King, K. C. Sole, and W. G. Davenport, Extractive Metallurgy of Copper, 2011, Elsevier Science.

101. A. Ante, Production of Marketable Gypsum from Weak Waste Acids - Saving Disposal Volume and Costs. Erzmetall, 2005. 2(58): p. 75-82.

102. A. Ante and S. Schonbrunner, Possible use of weak acid gypsum from the BAMAG process. Zkg International, 2007. 60(8): p. 59-68.

103. J. L. Huisman, Biologically produced sulphide for purification of process streams, effluent treatment and recovery of metals in the metal and mining industry. Hydrometallurgy, 2006. 83(1-4): p. 106-113.

104. C. M. Romo-Kröger, J. R. Morales, M. I. Dinator, F. Llona, and L. C. Eaton, Heavy-Metals in the Atmosphere Coming from a Copper Smelter in Chile. Atmospheric Environment, 1994. 28(4): p. 705-711.

105. C. M. Romo-Kröger and F. Llona, A Case of Atmospheric Contamination at the Slopes of the Los-Andes Mountain-Range. Atmospheric Environment Part a-General Topics, 1993. 27(3): p. 401-404.

106. G. Lagos, J. M. Lehuede, and M. Andia, Sulfur dioxide abatement costs and compliance with health-based standards: the case of copper smelters. Resources Policy, 2001. 27(3): p. 147-155. 
107. A. Roca, A. Morales, M. Cruells, and R. Bergo, Treatment of copper flash smelter flue dusts for copper and zinc extraction and arsenic stabilization. Hydrometallurgy, 2010. 105(1-2): p. 148-154.

108. C. Nunez, F. Espiell, and A. Roca, Recovery of Copper, Silver and Zinc from Huelva (Spain) Copper Smelter Flue Dust by a Chloride Leach Process. Hydrometallurgy, 1985. 14(1): p. 93-103.

109. M. Czaplicka and L. Buzek, Lead Speciation in the Dusts Emitted from NonFerrous Metallurgy Processes. Water Air and Soil Pollution, 2011. 218(1-4): p. $157-163$.

110. W. G. Davenport, M. King, A.K. Biswas, and M. Schlesinger, Extractive metallurgy of copper, 2002, Pergamon.

111. T. Inami, K. Baba, and Y. Ojima, Clean and high productive operation at the Sumitomo Toyo smelter, 1990. Sixth International Flash Smelting Congress, Brazil.

112. B. Lottermoser, Mine wastes : characterization, treatment and environmental impacts. 3rd ed, 2010, Springer.

113. V. Ettler, Z. Johan, A. Baronnet, F. Jankovskya, C. Giller, M. Mihaljevic, O. Sebek, L. Strnad, and A. Bezdicka, Mineralogy of Air-Pollution-Control Residues from a Secondary Lead Smelter: Environmental Implications. Environmental Science and Technology, 2005. 39: p. 9309-9316.

114. D. O. Cooney, Adsorption Design for Wastewater Treatment, 1999, Lewis Publishers.

115. R. H. Perry and J. H. Perry, Chemical engineers' handbook. 4th ed., 1963, McGraw-Hill.

116. C. Fonseca-Paris, Estudio de la extracción de Cu(II) y Zn(II) desde soluciones acuosas ácidas mediante el empleo de membranas líquidas emulsificada, microencapsulación de extractantes y silicatos de calcio nanoestructurados, 2009, Universidad de Chile.

117. Sepragen Corporation, Radial Flow Column, cited 2012, Available from: http://www.sepragen.com/page/Products-Chromatography-Columns.aspx.

118. T. Sparks, Solid-Liquid Filtration: A User's Guide to Minimizing Cost \& Environmental Impact, Maximizing Quality \& Productivity, 2011, Elsevier Science. 
119. A. Gedeon, P. Massiani, and F. Babonneau, Zeolites and Related Materials: Trends, Targets and Challenges : Proceedings of the 4th International FEZA Conference, Paris, France, 2-6 September, 2008, Elsevier Science Limited.

120. F. Bakhtiari and E. Darezereshki, One-step synthesis of tenorite $(\mathrm{CuO})$ nanoparticles from $\mathrm{Cu}_{4}\left(\mathrm{SO}_{4}\right)(\mathrm{OH})_{6}$ by direct thermal-decomposition method. Materials Letters, 2011. 65(2): p. 171-174.

121. P. E. de Jongh, D. Vanmaekelbergh, and J. J. Kelly, $\mathrm{Cu}_{2} \mathrm{O}$ : a catalyst for the photochemical decomposition of water?, Chemical Communications, 1999(12): p. 1069-1070.

122. G. B. Alexander, W. M. Heston, and R. K. Iler, The Solubility of Amorphous Silica in Water. Journal of Physical Chemistry, 1954. 58(6): p. 453-455. 


\section{Appendix}

A-1: Linear fit for the Langmuir adsorption isotherm data shown in Figure 3.30...212

A-2:Linear fit for the Freundlich adsorption isotherm data shown in Figure 3.30. .212

A-3: solubility of amorphous silica against $\mathrm{pH}$ at $298 \mathrm{~K}$ [122].

A-4: Diagram for oxidized and low grade copper sulfides processing in large scale mines in Chile [87] 214

A-5: Diagram for high grade copper sulfides processing in large scale mining in Chile [87]

A-6: Diagram for Lixiviation - Solvent Extraction - Electrowinning plants for the purification of copper [87]

A-7: Diagram for a electrorefining plant for copper cathode production [87]

A-8: Settling pond for the treatment of mining tailings [99]

A-9: Copper smelter effluent (weak acid) treatment [101]

A-10: BAMAG GmbH process for treating weak acid mining waste [101] 220

A-11: Biologically produce sulfur technology for mining waste water treatment [103]

A-12: Control chart showing As content in the weak acid of Plant 1 over the period of time studied.

A-13: Control chart showing sulfate content in the weak acid of Plant 1 over the period of time studied. 
A-14: Control chart showing the average amount of suspended solids present in the weak acid of Plant 1 over the period of time studied.

A-15: Control chart showing the As content in the weak acid of Plant 2 over the period of time studied. 223

A-16: Control chart showing the sulfate content in the weak acid of Plant 2 over the period of time studied. 224

A-17: Control chart showing the amount of suspended solids present in the weak acid of Plant 2 over the period of time studied.

A-18, Hidrolab ltda. report for the liquid portion of the weak acid collected at Caletones smelter, El Teniente mine, Rancagua, Chile.

A-19: Coupled process for the production of clean gypsum and the recovery of $\mathrm{Cu}^{2+}$ using NCaSil.

A-20: Table showing values of the shape factor of different materials. Values for nearly spherical particles are close to 1 , while high asymmetry in the shape makes the shapes approach 0 . Table taken from reference [115]. 227

A-21: Graphical calculation to obtained modified friction factor and $\mathrm{n}$ value for modified Reynolds number values from 10-3 to 106. Graph taken from reference $[115]$

A-22:SEM image in normal and backscatter mode of the geopolymer surface sample containing tenorite nanoparticles. Nanoparticles appear as bright spots on the image taken in backscatter mode. The material shows a large pores ranging 0.1 to $1 \mu \mathrm{m}$ in diameter. 228 


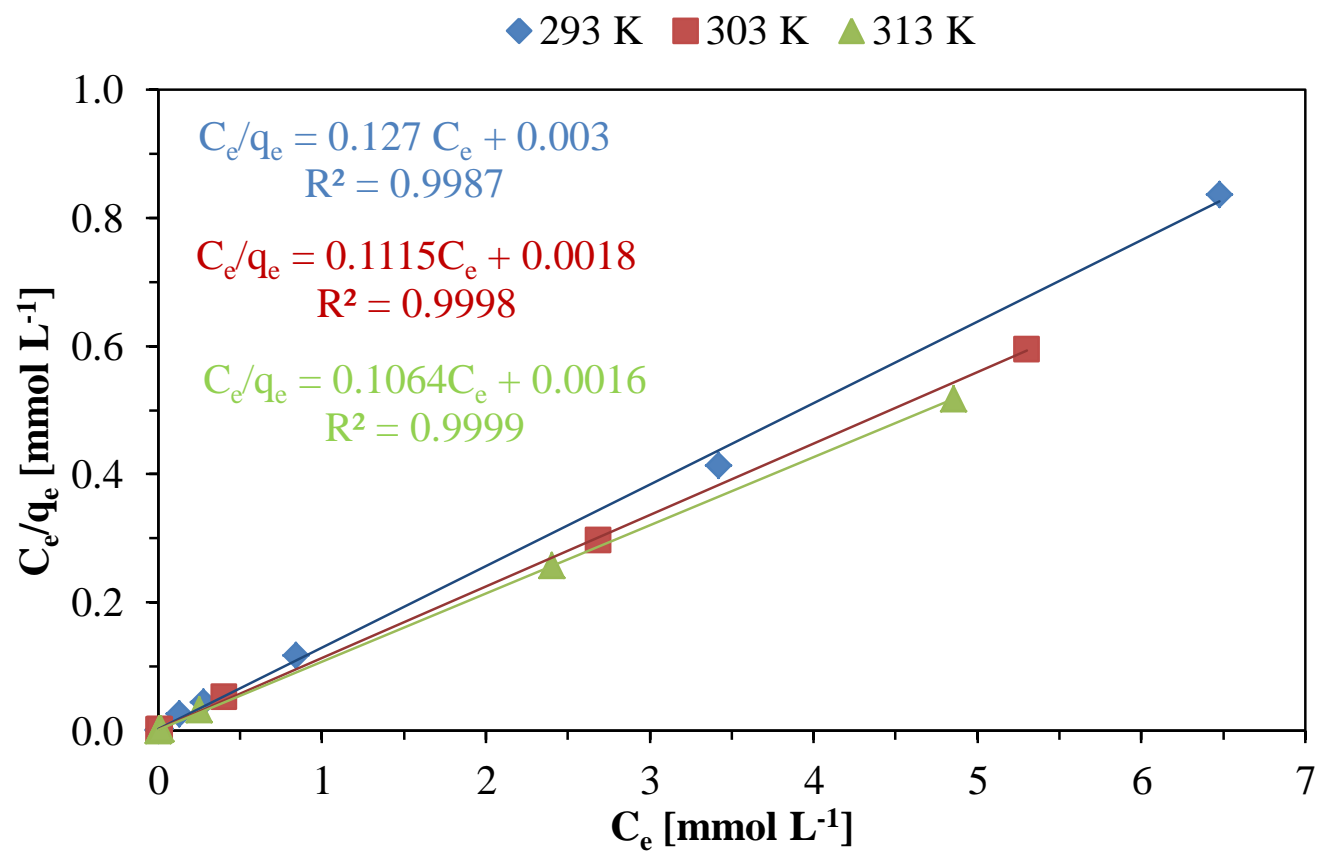

A-1: Linear fit for the Langmuir adsorption isotherm data shown in Figure 3.30.

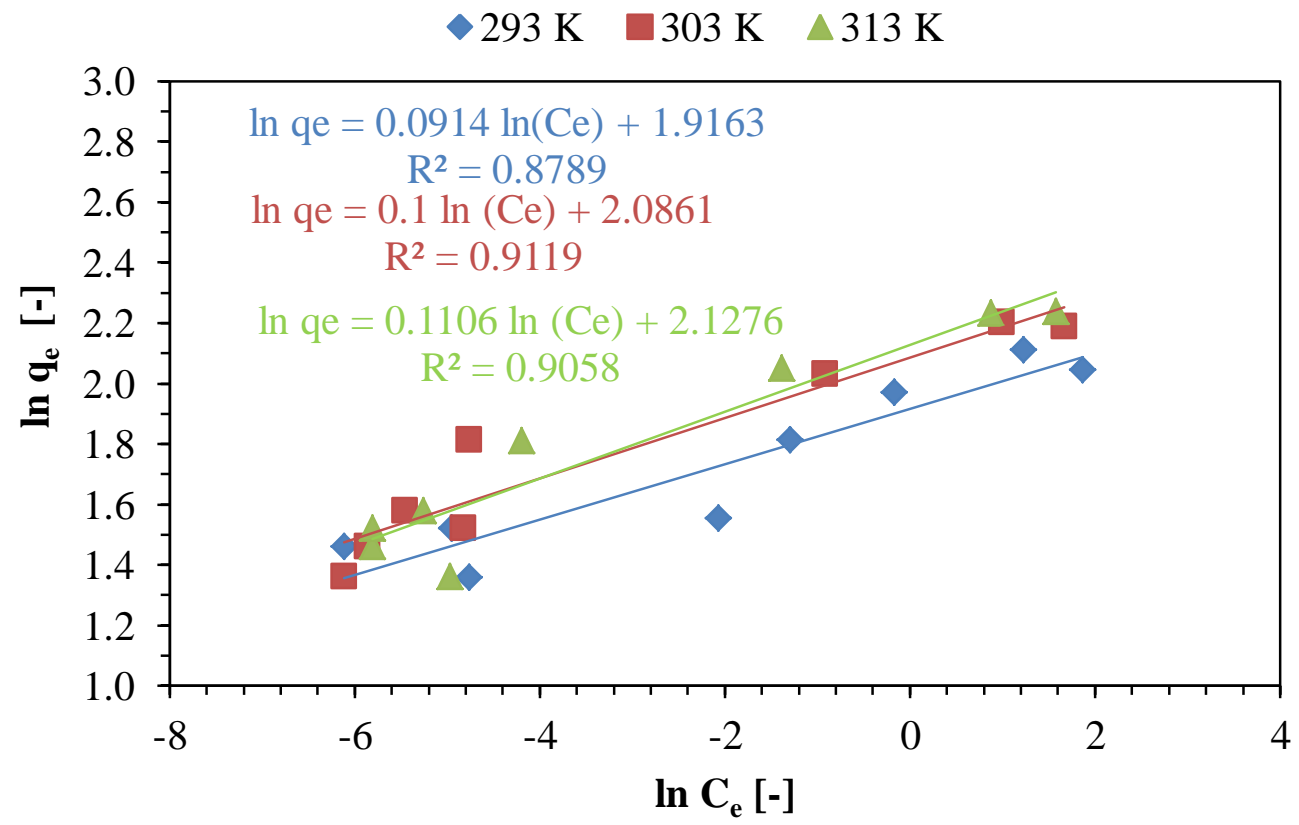

A-2:Linear fit for the Freundlich adsorption isotherm data shown in Figure 3.30. 


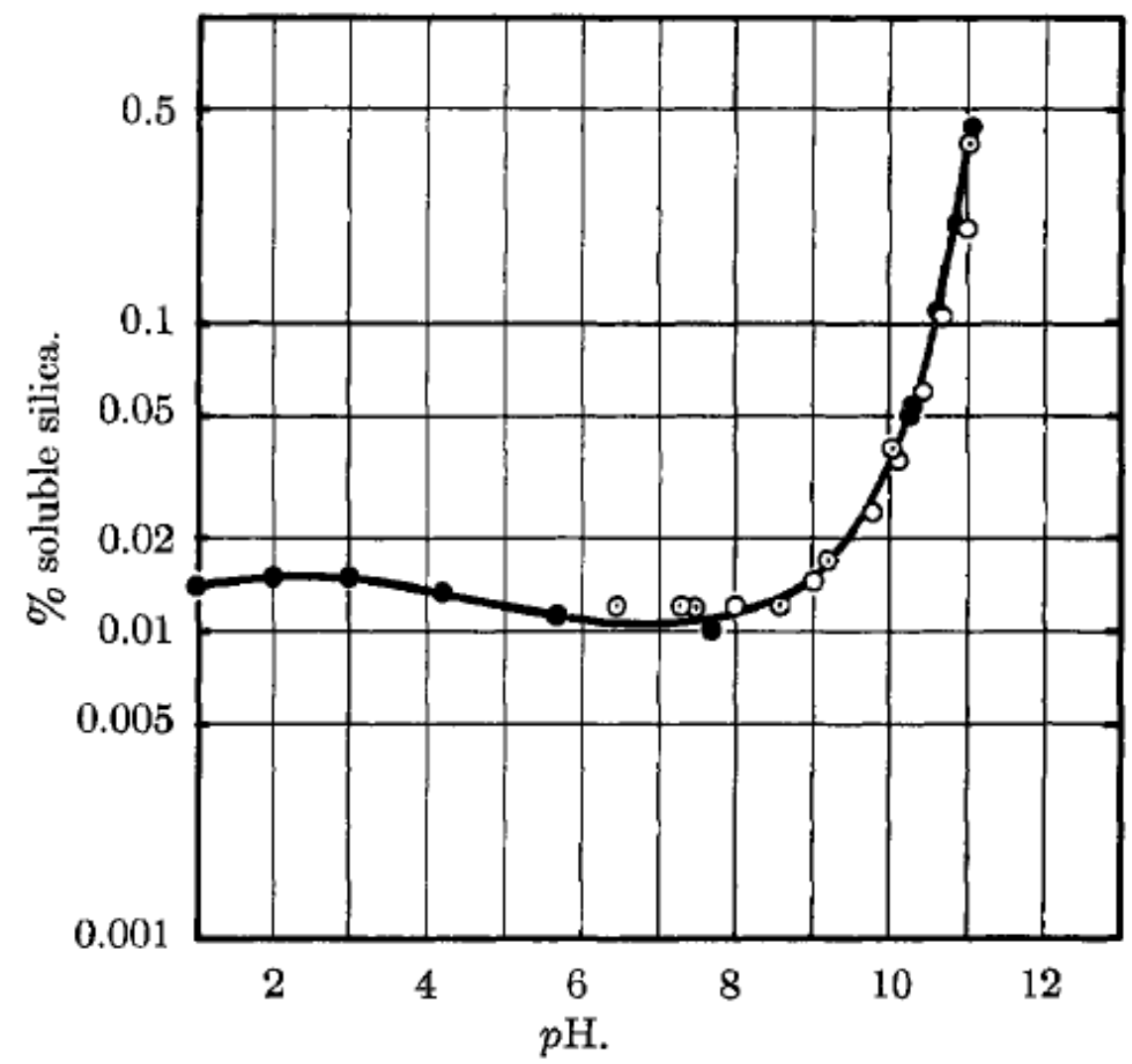

A-3: solubility of amorphous silica against pH at $298 \mathrm{~K}$ [122]. 


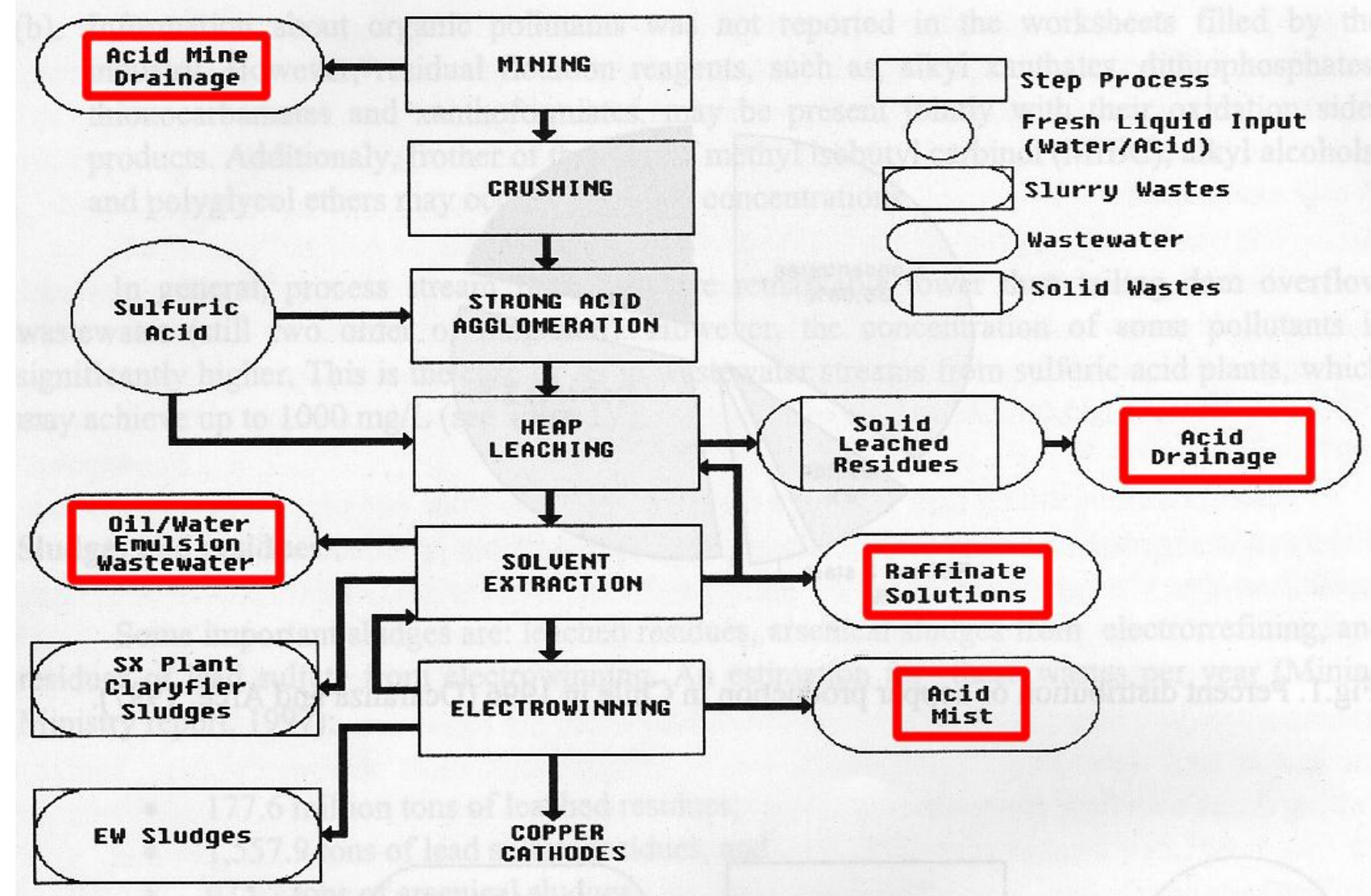

A-4: Diagram for oxidized and low grade copper sulfides processing in large scale mines in Chile [87] 


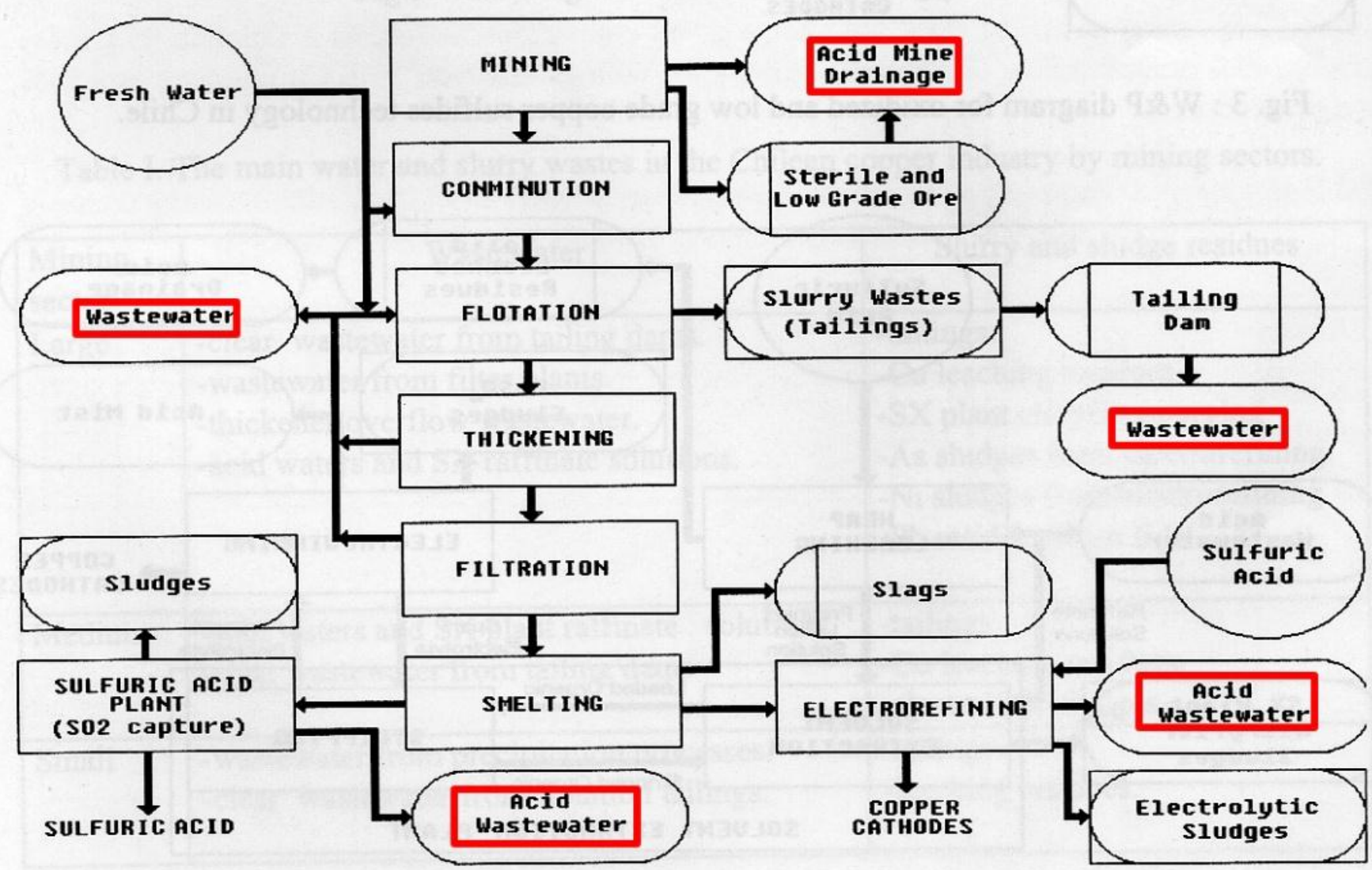

A-5: Diagram for high grade copper sulfides processing in large scale mining in Chile [87] 


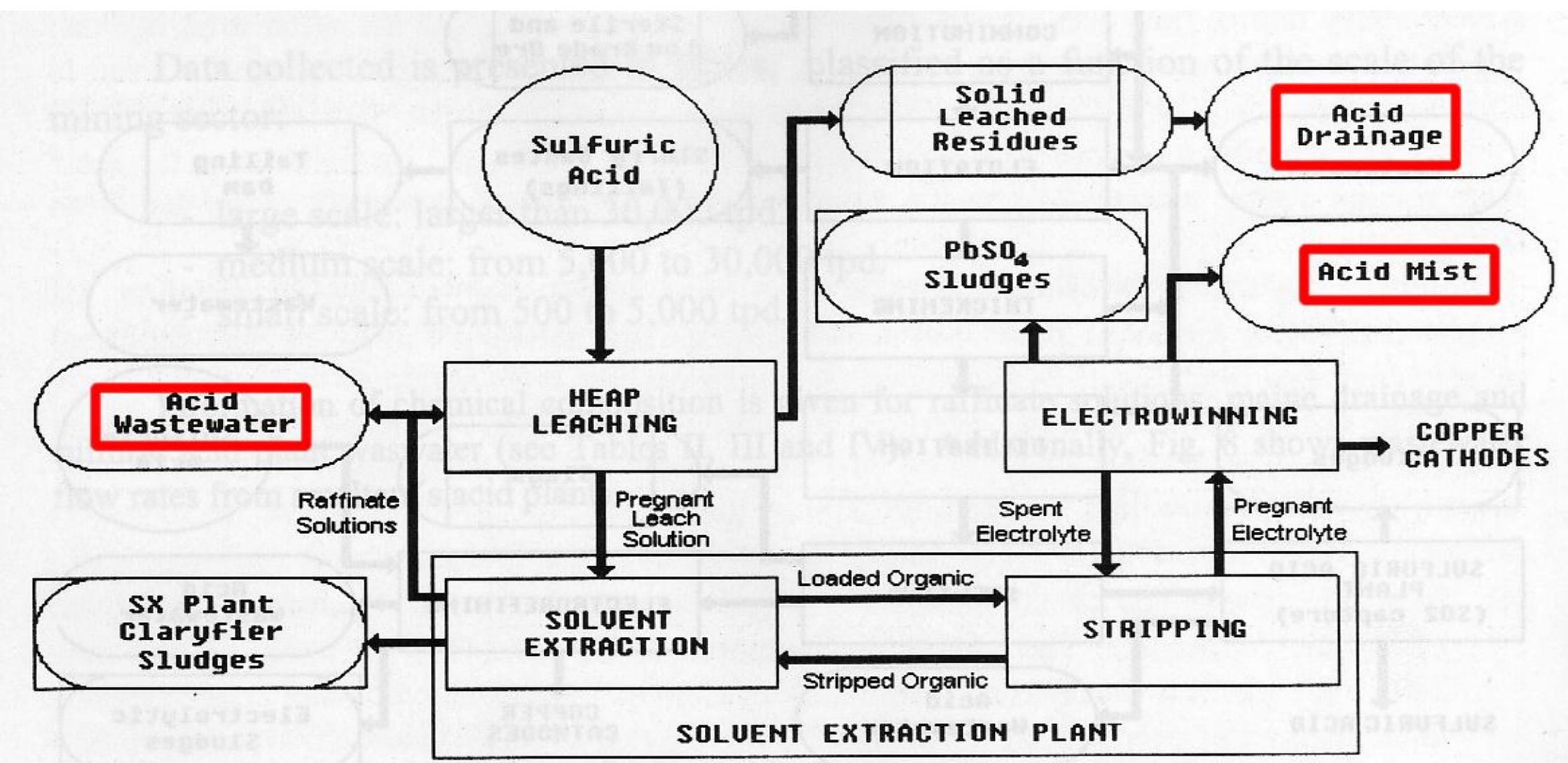

A-6: Diagram for Lixiviation - Solvent Extraction - Electrowinning plants for the purification of copper [87] 


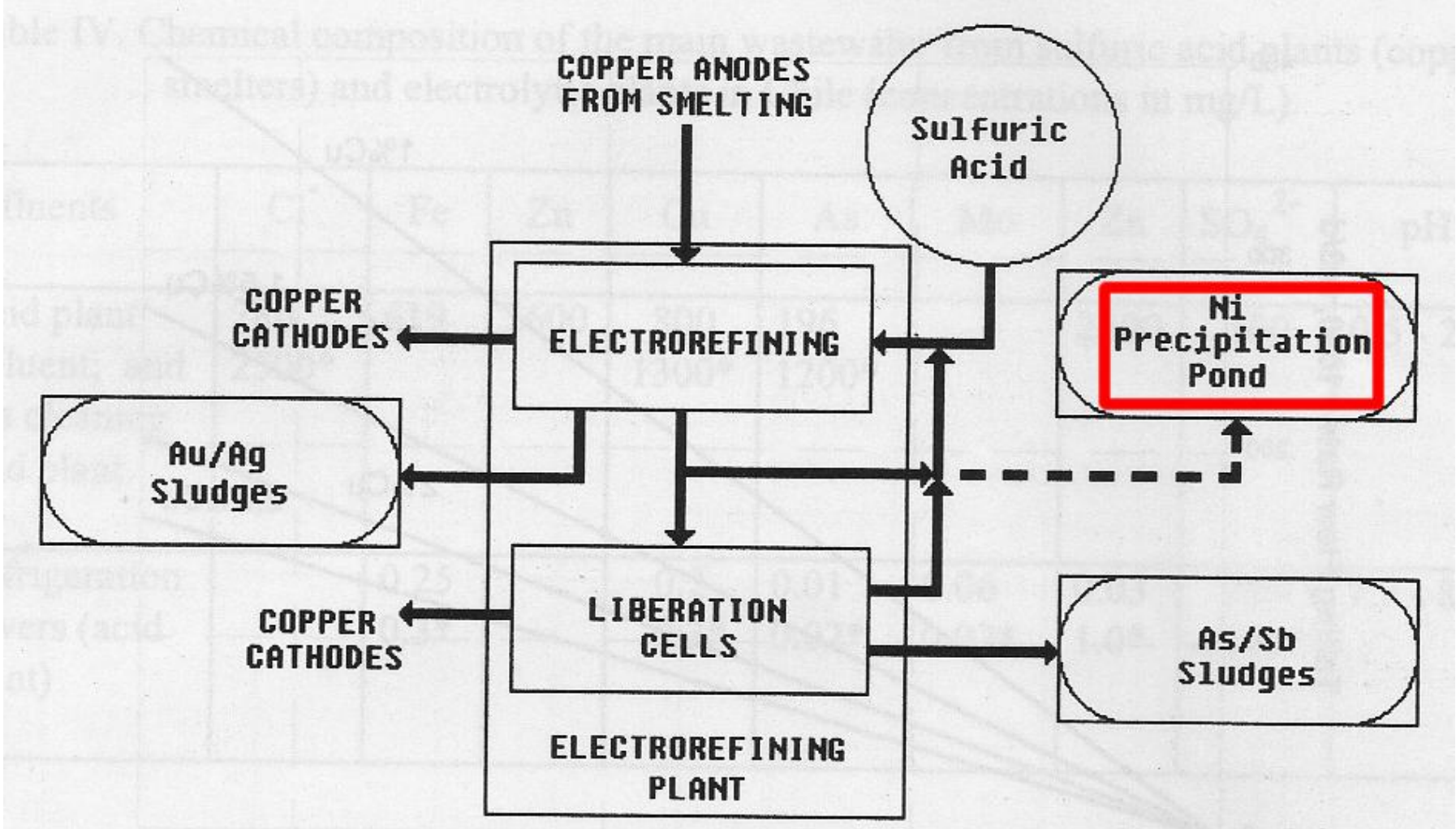

A-7: Diagram for a electrorefining plant for copper cathode production [87] 


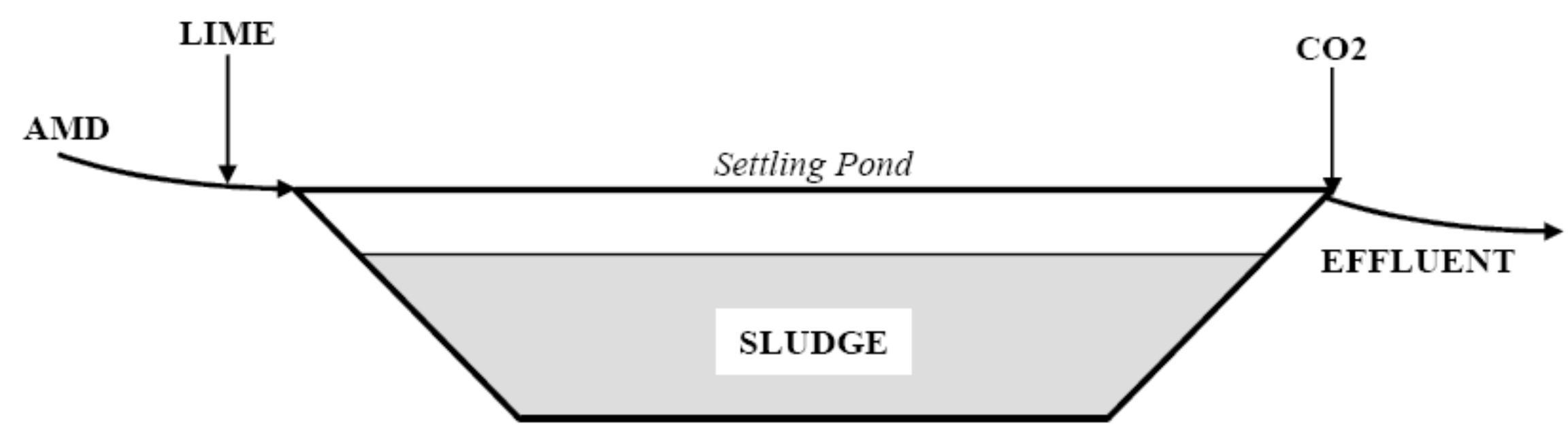

A-8: Settling pond for the treatment of mining tailings [99] 


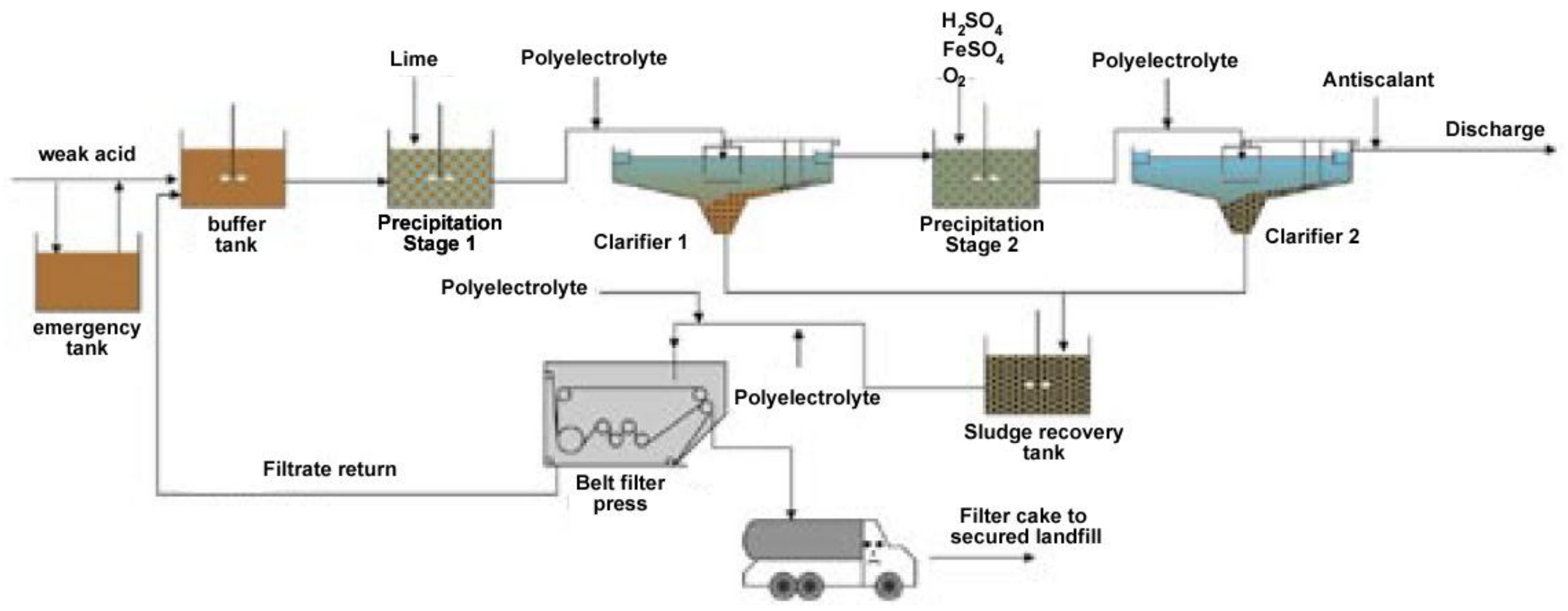

A-9: Copper smelter effluent (weak acid) treatment [101] 


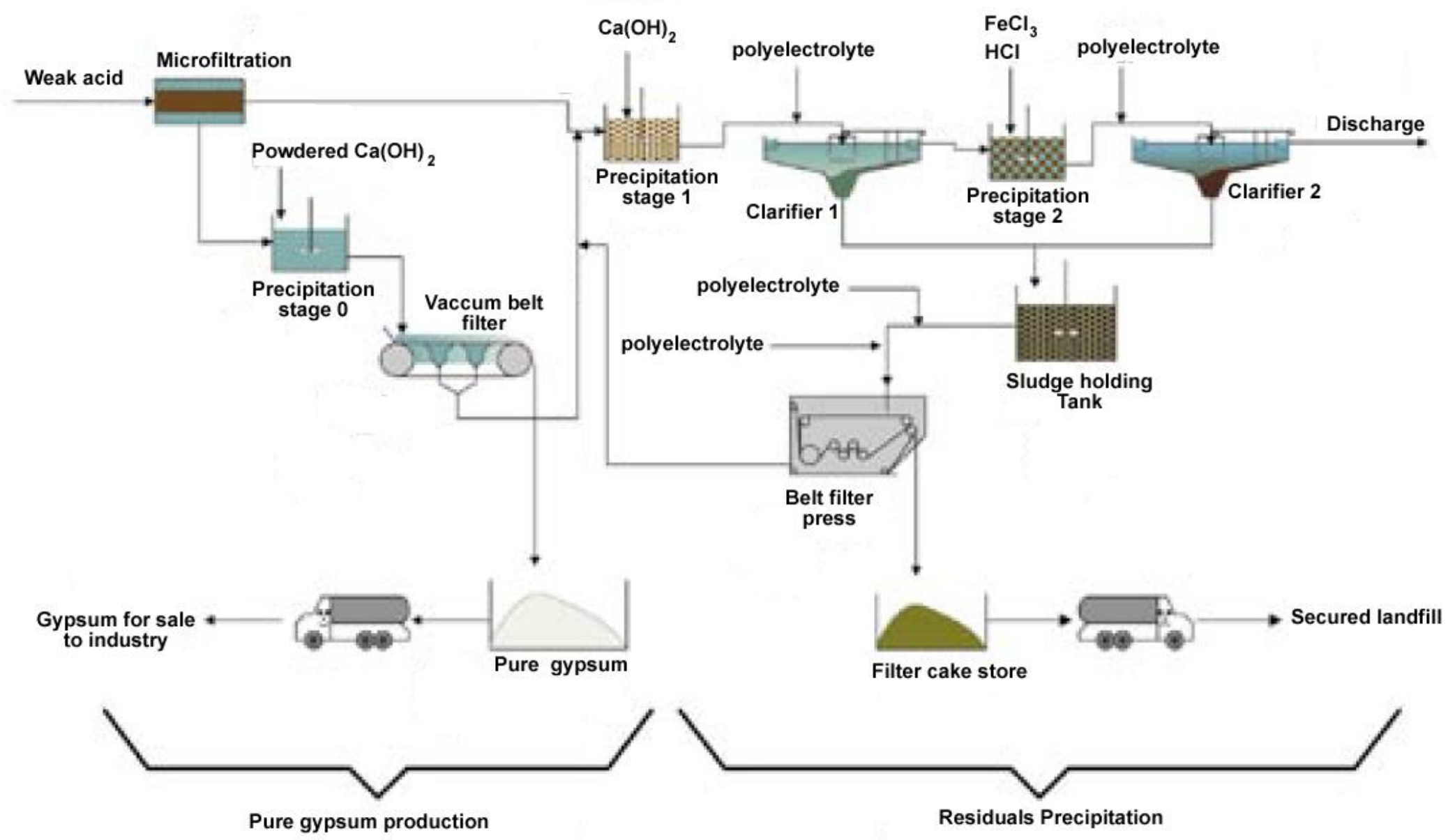

A-10: BAMAG GmbH process for treating weak acid mining waste [101] 


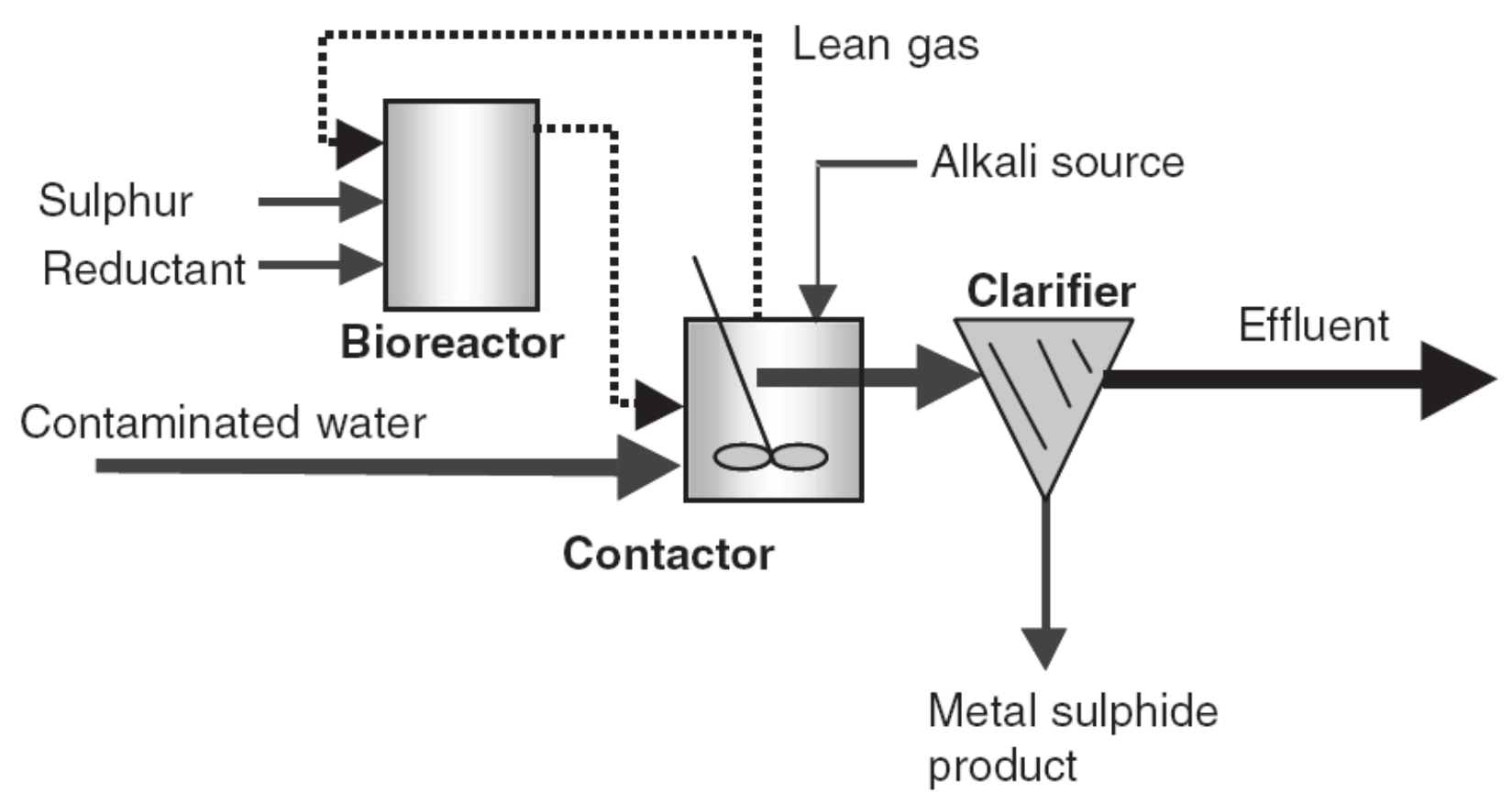

A-11: Biologically produce sulfur technology for mining waste water treatment [103] 


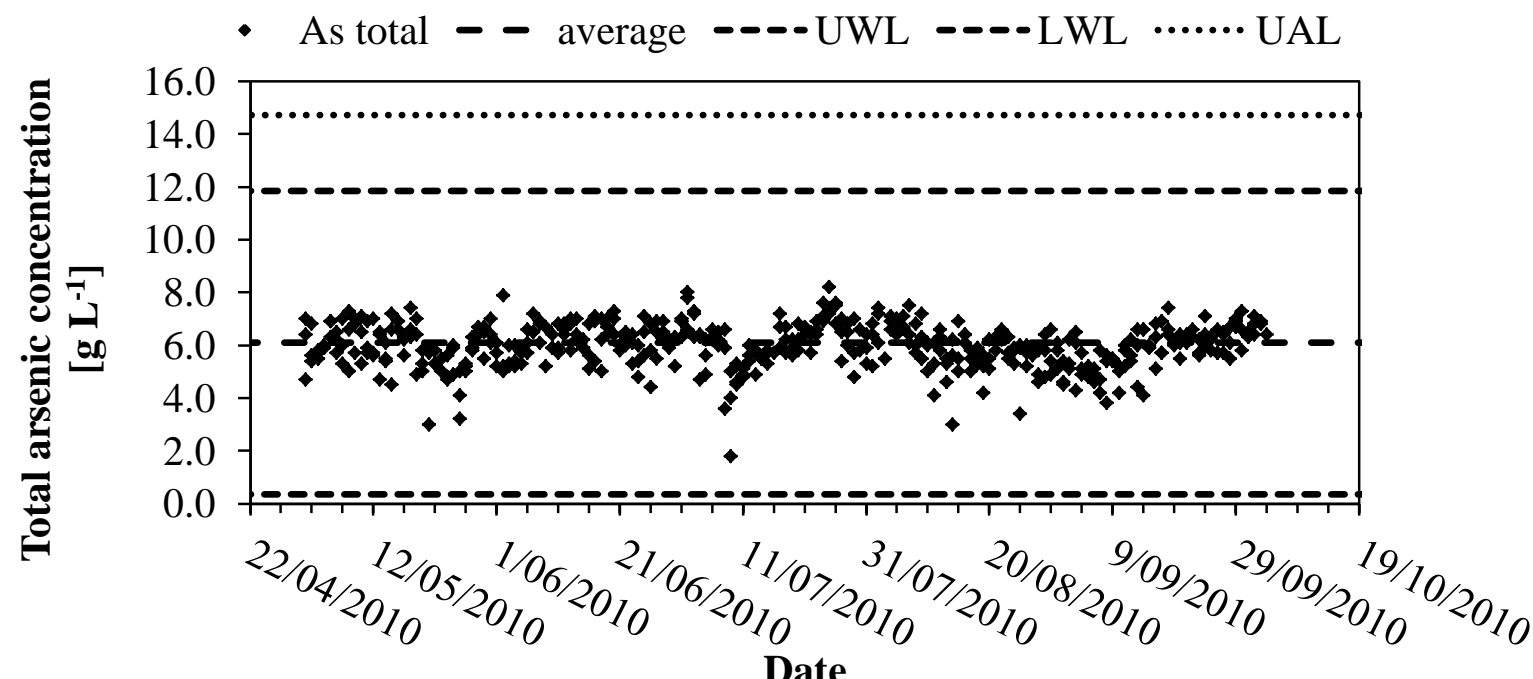

A-12: Control chart showing As content in the weak acid of Plant 1 over the period of time studied.

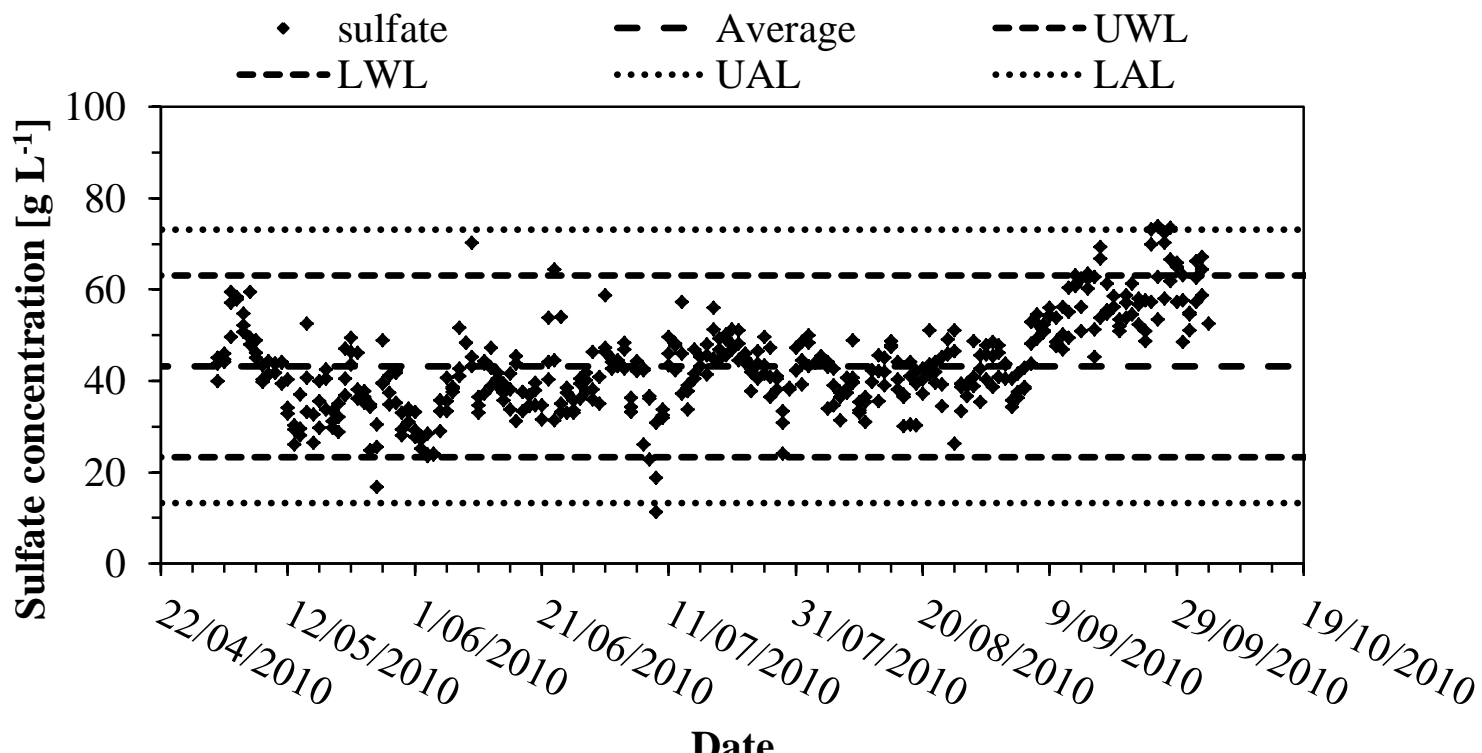

A-13: Control chart showing sulfate content in the weak acid of Plant 1 over the period of time studied. 


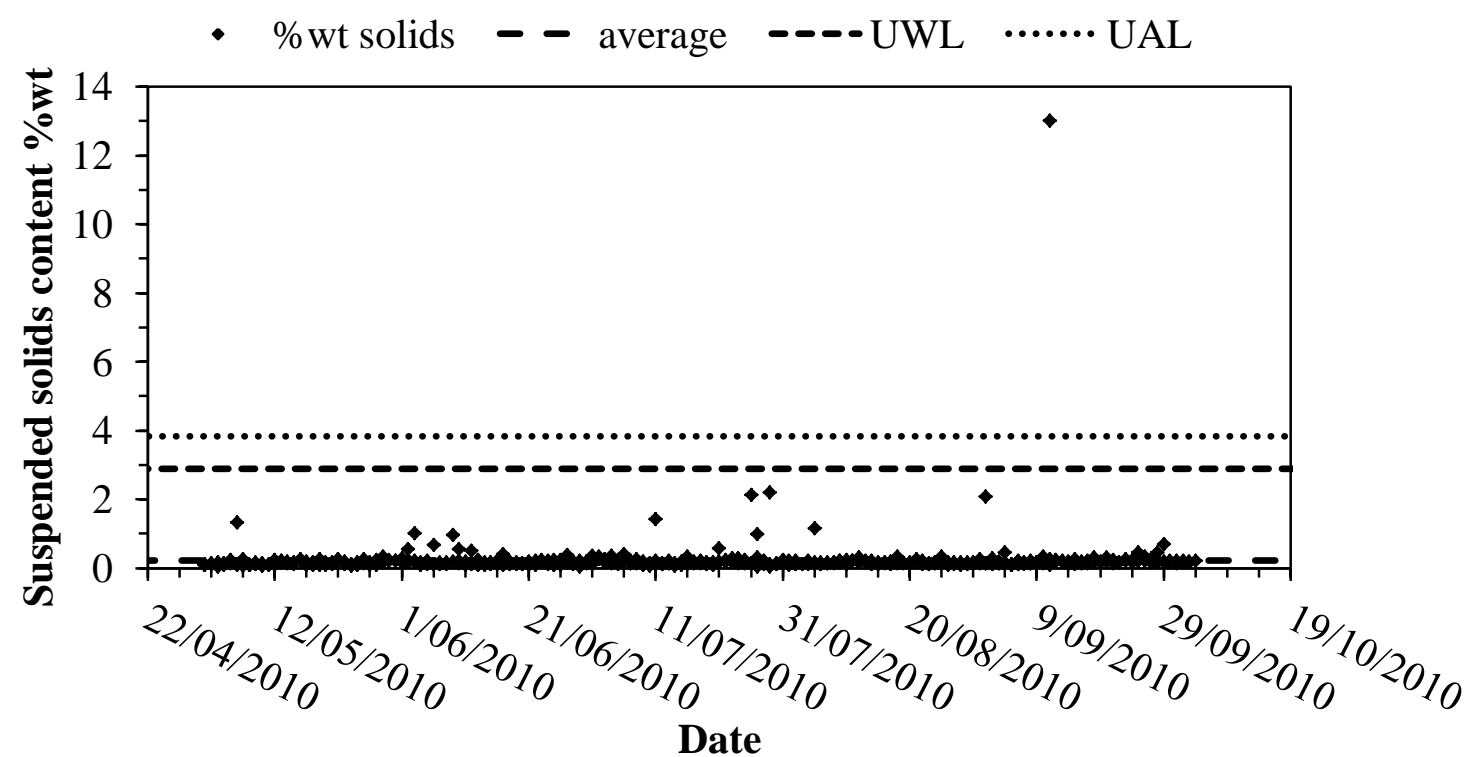

A-14: Control chart showing the average amount of suspended solids present in the weak acid of Plant 1 over the period of time studied.

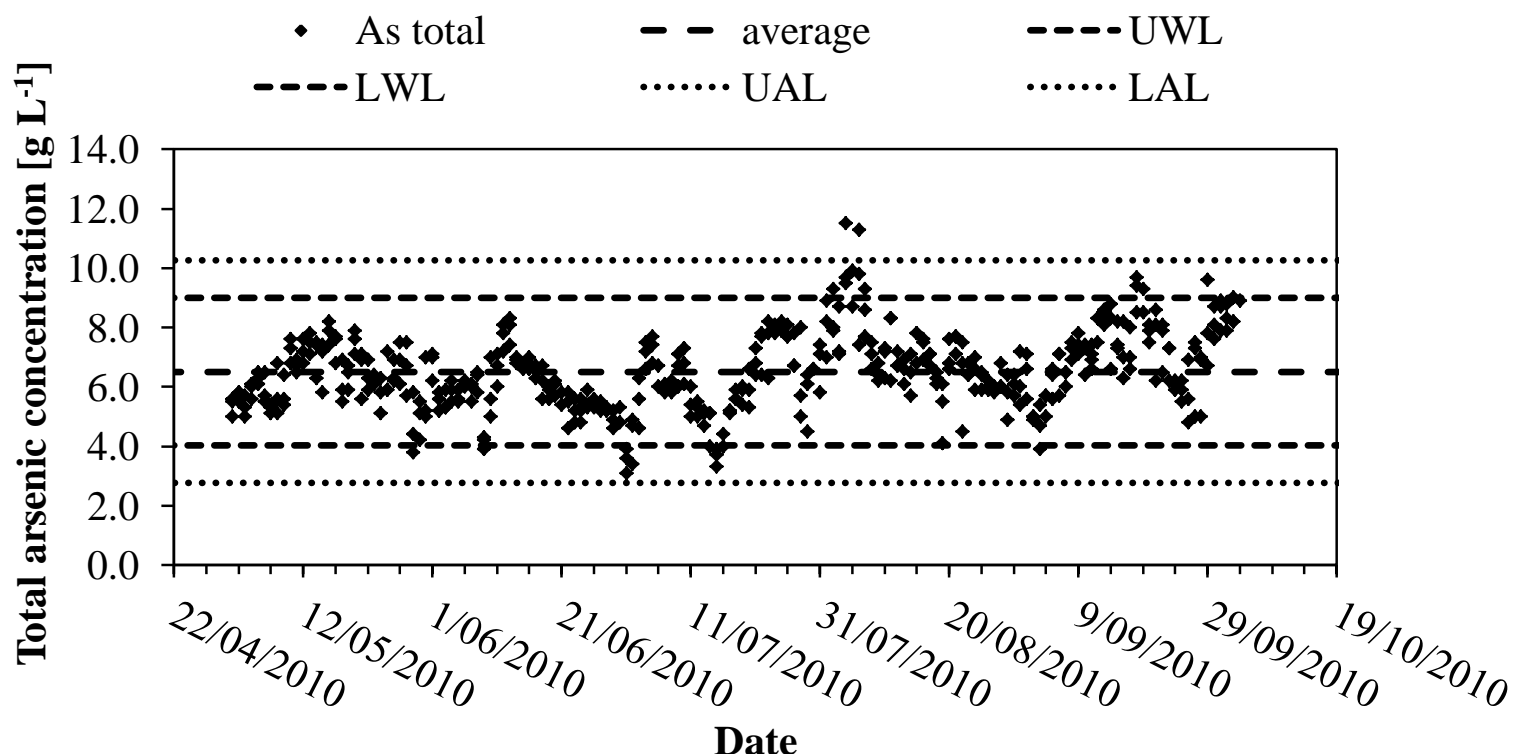

A-15: Control chart showing the As content in the weak acid of Plant 2 over the period of time studied. 


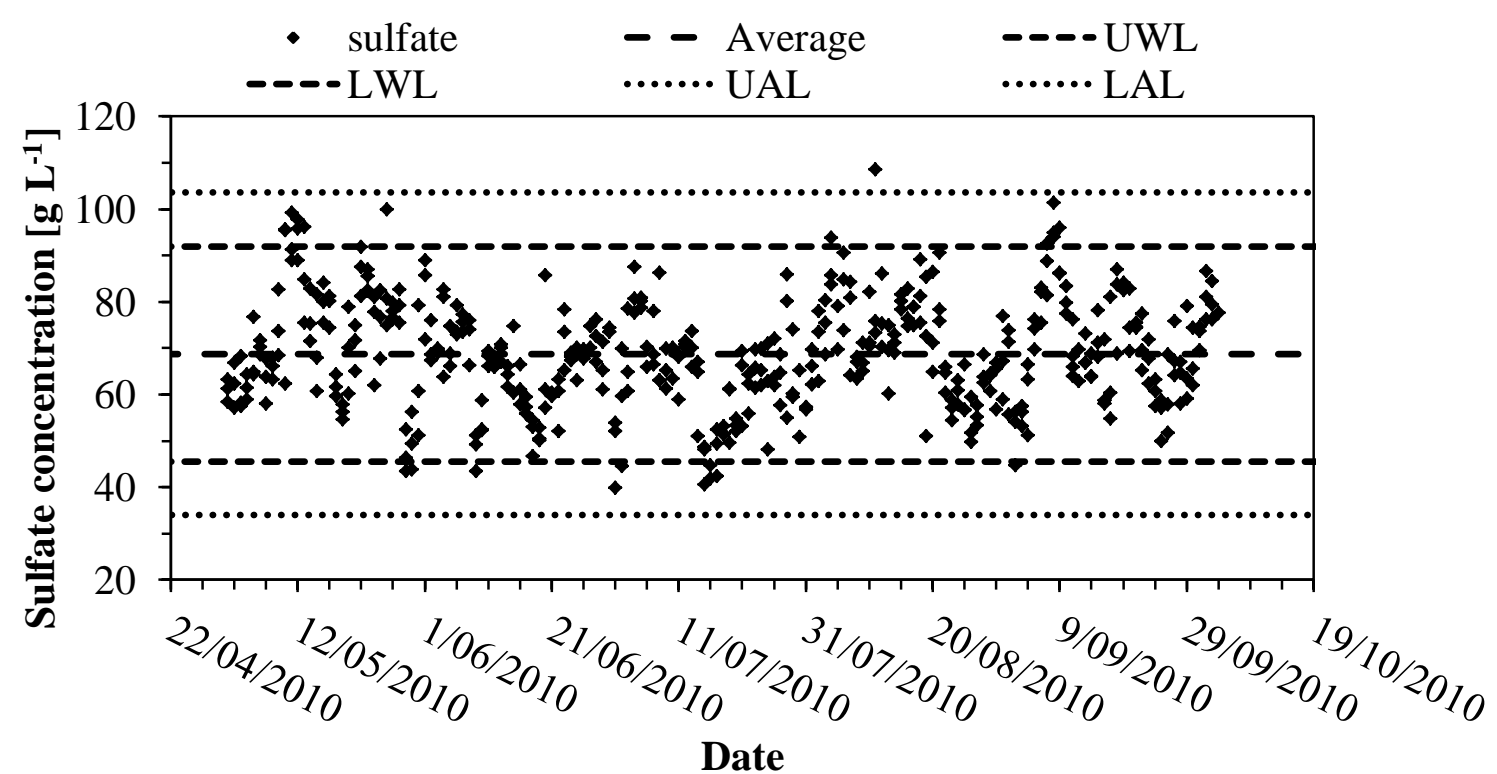

A-16: Control chart showing the sulfate content in the weak acid of Plant 2 over the period of time studied.

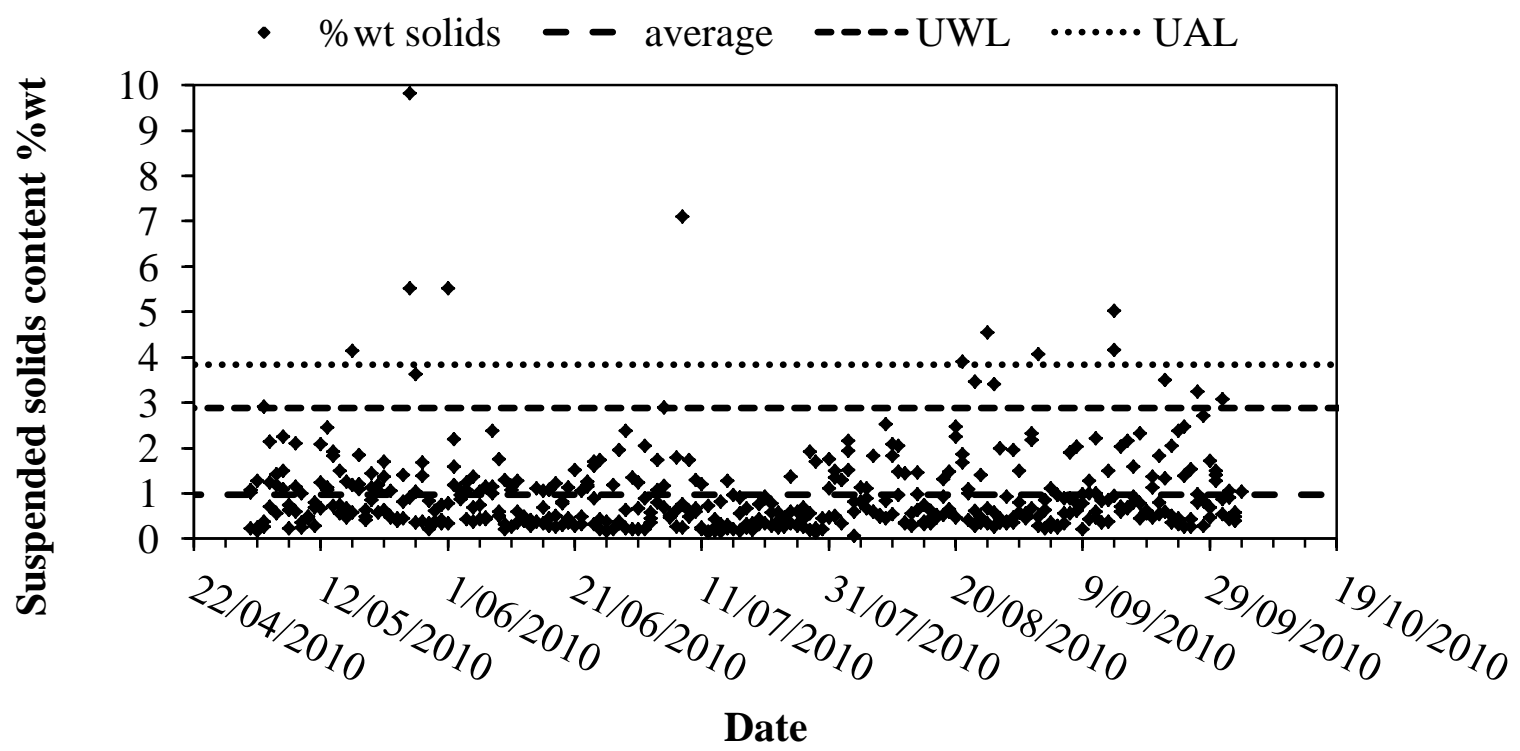

A-17: Control chart showing the amount of suspended solids present in the weak acid of Plant 2 over the period of time studied. 
Informe de Ensayo

N'Informe: 68880-01

Proyecto: Control Muestra de RILes

Identificación Cliente: Torre de Lavado

Lugar de Muestreo: Mina Teniente

Dirección: Fundición Caletones

Cindad / Región: Rancagua, Sexta Región

Punto de Muestreo: Torre de Lavado

Matríz: RILes

Tipo de Muestreo: Puntual

Término de Muestreo: 29/08/2010 11:00:00

Recepción Laboratorio: 30/08/2010 16:54:18

Muestreado por: Cliente

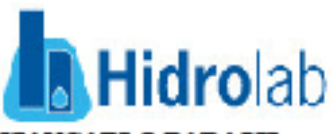

GIANCARIO BARASSI

Dirección:

AVDA AMERICO VESPUCIO NORTE 280 DEPTO

1303, Las Condes,

\begin{tabular}{|c|c|c|c|c|}
\hline Parámetro & Unidades & Resultados & $\begin{array}{c}\text { Fecha y Hora } \\
\text { Análisis }\end{array}$ & Ref.Método \\
\hline Clonuros & $\mathrm{mg} / \mathrm{L} \mathrm{Cl}$ & 475 & $31 / 08 / 1014: 10$ & $2313-32009(1)$ \\
\hline Nitrato & $\mathrm{mg} / \mathrm{L} \mathrm{N}-\mathrm{NO} 3$ & $<0,20$ & $30 / 08 / 1017: 21$ & $\mathrm{SM}-4110 \mathrm{~B}(2)$ \\
\hline $\mathrm{pH}$ & umidad & $0,45\left(21,0^{\circ} \mathrm{C}\right)$ & $31 / 08 / 1017: 04$ & 2313-lof $5(1)$ \\
\hline Sulfato disuelto & $\mathrm{mg} / \mathrm{L}$ SO4 & 64518 & $31 / 08 / 1015: 05$ & $2313-18097(1)$ \\
\hline Aluminio & $\mathrm{mg} / \mathrm{L} \mathrm{Al}$ & 113 & $06 / 09 / 1012: 05$ & $2313-25 \circ 97(1)$ \\
\hline Arsénico & $\mathrm{mg} / \mathrm{L}$ As & 0,146 & $07 / 09 / 1016: 21$ & $2313-90 f 96(1)$ \\
\hline Calcio & $\mathrm{mg} / \mathrm{L} \mathrm{Ca}$ & 1449 & 03/09/10 11:52 & SM-3111B(2) \\
\hline Cobalto & $\mathrm{mg} / \mathrm{L} \mathrm{Co}_{0}$ & 0,103 & $06 / 09 / 1012: 09$ & $2313-25097(1)$ \\
\hline Cromo & $\mathrm{mg} / \mathrm{LCr}$ & 0,076 & $06 / 09 / 1012: 11$ & $2313-25097(1)$ \\
\hline Cobre & $\mathrm{mg} / \mathrm{LCu}$ & 562 & $06 / 09 / 1012: 10$ & $2313-25097(1)$ \\
\hline Hierro & $\mathrm{mg} / \mathrm{L} \mathrm{Fe}$ & 185 & $06 / 09 / 1012: 12$ & $2313-25097(1)$ \\
\hline Potasio & $\mathrm{mg} / \mathrm{LK}$ & 467 & 03/09/10 12:14 & SM-3111B(2) \\
\hline Magnesio & $\mathrm{mg} / \mathrm{L} \mathrm{Mg}$ & 39,7 & $03 / 09 / 1012: 47$ & SM-3111B(2) \\
\hline Molibdeno & mg/L Mo & 50,3 & $06 / 09 / 1012: 13$ & $2313-25 \circ 97(1)$ \\
\hline Sodio & $\mathrm{mg} / \mathrm{L} \mathrm{Na}$ & 152 & 03/09/10 14:40 & SM-3111B(2) \\
\hline Niquel & $\mathrm{mg} / \mathrm{L} \mathrm{Ni}$ & 0,402 & $06 / 09 / 1012: 13$ & $2313-25 \circ 97(1)$ \\
\hline Plomo & $\mathrm{mg} / \mathrm{L} \mathrm{Pb}$ & 22,0 & $06 / 09 / 1012: 14$ & $2313-25097(1)$ \\
\hline Vanadio & $\mathrm{mg} / \mathrm{L}$ & $<0,008$ & $06 / 09 / 1012: 15$ & $2313-25097(1)$ \\
\hline DQO & $\mathrm{mg} / \mathrm{L}$ & 3023 & $03 / 09 / 1012: 35$ & $2313-24097(1)$ \\
\hline Acidez & $\mathrm{mg} / \mathrm{L} \mathrm{CaCO} 3$ & 2970 & $06 / 09 / 1016: 40$ & $\mathrm{SM}-2310 \mathrm{~B}(2)$ \\
\hline Conductividad & $\mathrm{us} / \mathrm{cm}$ & 334000 & $31 / 08 / 1014: 16$ & $\mathrm{SM}-2510 \mathrm{~B}(2)$ \\
\hline
\end{tabular}

(1) Normas Chilenas Oficializadas, serie NCh 2313 - Residuos Industriales Líquidos.

(2) Standard Methods for the Examination of Water and Wastewater, 21th Edition 2005.

$\mathrm{pH}$ medido en el laboratorio

HIIDROLAB se encuentra bajo las Acreditaciones INN LE 214-LE 215-LE 216-LE 217; de acuerdo a NCh-ISO 17025 Of 2005

Avenida Central No 681 - Quilicura - Teléfono: 756 6350 - Fax: 7566351 www hidrolab.cl - email: laboratorio $a$ hidrolab.cl

A-18, Hidrolab Itda. report for the liquid portion of the weak acid collected at Caletones smelter, El Teniente mine, Rancagua, Chile. 

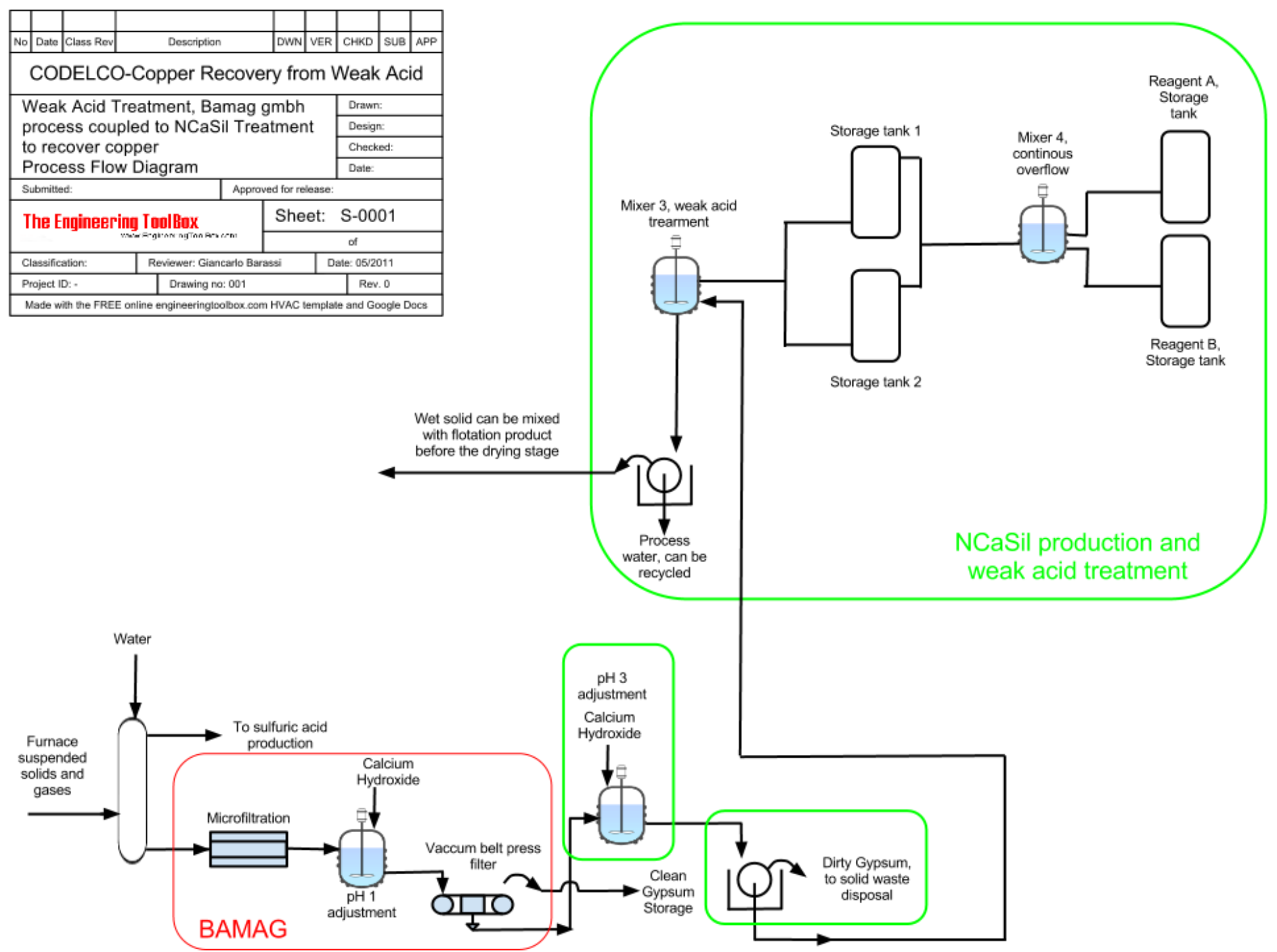

A-19: Coupled process for the production of clean gypsum and the recovery of $\mathrm{Cu}^{2+}$ using $\mathrm{NCaSil}$. 


\section{Shape Factors for Non-spherical Particles*}

\begin{tabular}{|c|c|c|}
\hline Material & Nature of grain & 6 \\
\hline rnould's wire spirals $\ldots \ldots \ldots \ldots \ldots \ldots \ldots \ldots$ & $\cdots \cdots \cdots$ & 0.2 \\
\hline$\because \cdots \cdots \cdots \cdots \cdots \cdots$ & $\cdots$ & 3 \\
\hline (up to $3 / 8$ in.) $\ldots \ldots \ldots \ldots \ldots$ & • & 6 \\
\hline$\cdots \cdots \cdots \cdots \cdots \cdots \cdots$ & $\cdots$ & it \\
\hline$\ldots \cdots, \cdots \cdots \cdots \cdots \cdots \cdots \cdots \cdots \cdots \cdots \cdots \cdots$ & Kus & \\
\hline$\ldots \ldots \ldots \ldots \ldots \ldots \ldots \ldots \ldots \ldots$ & Fus & \\
\hline$\cdots \cdots \cdots \cdots \cdots \cdots \cdots \cdots \cdots \cdots$ & Fused, agsregates & \\
\hline $\cdots \cdots \cdots \cdots \cdots \cdots \cdots \cdots \cdots \cdots \cdots \cdots$ & $i_{i} \cdots \cdots \cdots \cdots$ & \\
\hline$\cdots \cdots$ & $\ldots$ & \\
\hline Raschig rings. & & 3 \\
\hline & & \\
\hline$\cdots$ & Ja & \\
\hline i. & ed fla & \\
\hline nd. & Nearly spherical & \\
\hline & Rov & \\
\hline & An & \\
\hline & $J_{\mathrm{a}}$ & \\
\hline & & \\
\hline
\end{tabular}

A-20: Table showing values of the shape factor of different materials. Values for nearly spherical particles are close to 1 , while high asymmetry in the shape makes the shapes approach 0. Table taken from reference [115].

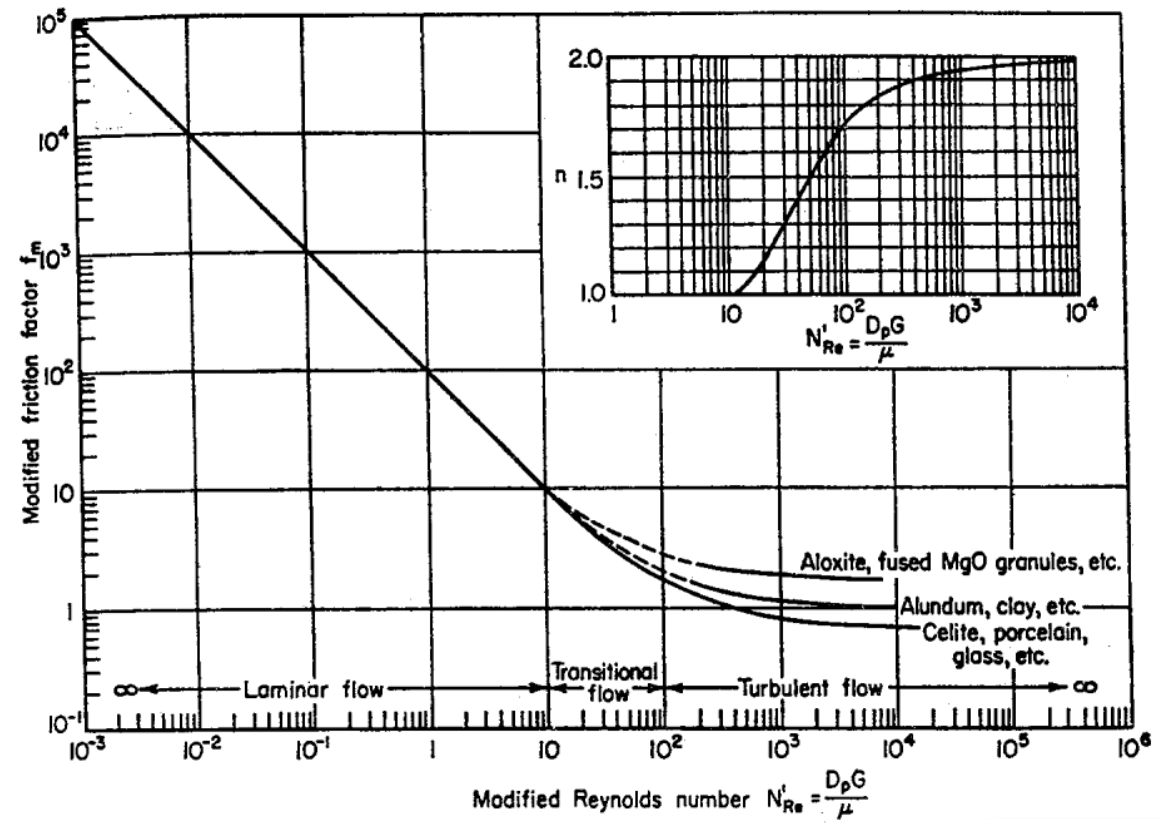

A-21: Graphical calculation to obtained modified friction factor and $\mathbf{n}$ value for modified Reynolds number values from 10-3 to 106. Graph taken from reference [115] 


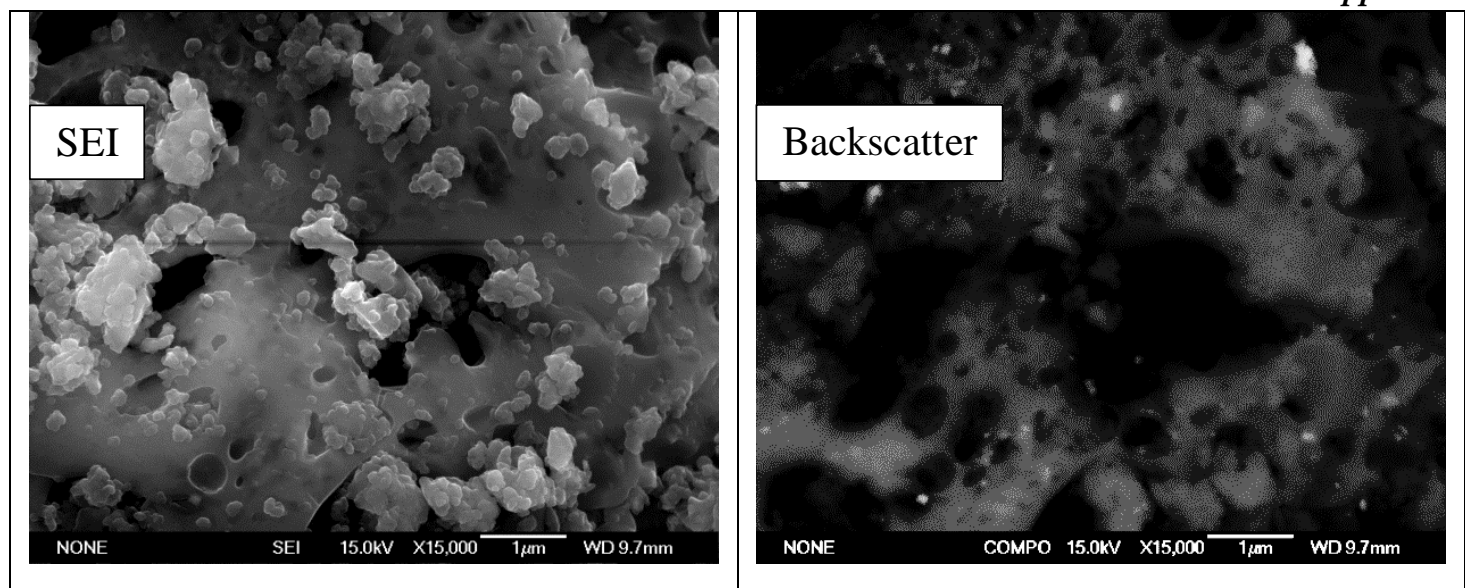

A-22:SEM image in normal and backscatter mode of the geopolymer surface sample containing tenorite nanoparticles. Nanoparticles appear as bright spots on the image taken in backscatter mode. The material shows a large pores ranging 0.1 to $1 \mu \mathrm{m}$ in diameter. 